\title{
Financial incentives for smoking cessation
}

Citation for published version (APA):

van den Brand, F. A. (2020). Financial incentives for smoking cessation. [Doctoral Thesis, Maastricht University]. ProefschriftMaken. https://doi.org/10.26481/dis.20201021fb

Document status and date:

Published: 01/01/2020

DOI:

10.26481/dis.20201021fb

Document Version:

Publisher's PDF, also known as Version of record

\section{Please check the document version of this publication:}

- A submitted manuscript is the version of the article upon submission and before peer-review. There can be important differences between the submitted version and the official published version of record.

People interested in the research are advised to contact the author for the final version of the publication, or visit the DOI to the publisher's website.

- The final author version and the galley proof are versions of the publication after peer review.

- The final published version features the final layout of the paper including the volume, issue and page numbers.

Link to publication

\footnotetext{
General rights rights.

- You may freely distribute the URL identifying the publication in the public portal. please follow below link for the End User Agreement:

www.umlib.nl/taverne-license

Take down policy

If you believe that this document breaches copyright please contact us at:

repository@maastrichtuniversity.nl

providing details and we will investigate your claim.
}

Copyright and moral rights for the publications made accessible in the public portal are retained by the authors and/or other copyright owners and it is a condition of accessing publications that users recognise and abide by the legal requirements associated with these

- Users may download and print one copy of any publication from the public portal for the purpose of private study or research.

- You may not further distribute the material or use it for any profit-making activity or commercial gain

If the publication is distributed under the terms of Article $25 \mathrm{fa}$ of the Dutch Copyright Act, indicated by the "Taverne" license above, 
Financial incentives for smoking cessation

Floor van den Brand 
(c) Floor van den Brand, Maastricht 2020

Cover design: In60seconds

Printing: ProefschriftMaken | proefschriftmaken.nl

ISBN 978-94-6380-964-1

The research in this dissertation was conducted at the Care and Public Health Research Institute (CAPHRI), Department of Family Medicine, Maastricht University. CAPHRI is part of The Netherlands School of Public Health and Care Research (CaRe). The research was funded by the Dutch Cancer Society (KWF Kankerbestrijding) and ZonMw. 


\title{
Financial incentives for smoking cessation
}

\author{
Proefschrift
}

Ter verkrijging van de graad van doctor aan de Universiteit Maastricht, op gezag van de Rector Magnificus, Prof. Dr. Rianne M. Letschert, volgens het besluit van het College van Decanen, in het openbaar te verdedigen op woensdag 21 oktober 2020 om 16.00 uur

door

Floortje Anne van den Brand

geboren op 3 december 1986 te Oosterhout 


\section{Promotores}

Prof. dr. ir. C. P. van Schayck

Prof. dr. G. E. Nagelhout

Prof. dr. N. H. Chavannes, LUMC

Co-promotor

Dr. Bjorn Winkens

\section{Beoordelingscommissie}

Prof.dr. J.A. Knottnerus (voorzitter)

Prof.dr. C. Bolman, Open Universiteit Heerlen

Prof.dr. J.P. Mackenbach, Erasmus Medisch Centrum Rotterdam

Prof.dr. N.K. de Vries

Prof.dr. H. de Vries 


\section{Contents}

CHAPTER 1 General introduction

CHAPTER 2 Healthcare financing systems for increasing the use of tobacco dependence treatment

CHAPTER 3 Does free or lower cost smoking cessation medication stimulate quitting? Findings from the International Tobacco Control (ITC) Netherlands and United Kingdom Surveys

CHAPTER 4 The effect of financial incentives on top of behavioral support on quit rates in tobacco smoking employees: study protocol of a clusterrandomized trial

CHAPTER 5 Effect of a workplace-based group training program combined with financial incentives on smoking cessation: a cluster-randomized controlled trial

CHAPTER 6 'Secretly, it's a competition': a qualitative study investigating what helped employees quit smoking during a workplace smoking cessation group training program with incentives

CHAPTER 7 The association of peer smoking behavior and social support with quit success in employees who participated in a smoking cessation intervention at the workplace

CHAPTER 8 How financial incentives increase smoking cessation: a two-level path analysis

CHAPTER 9 Cost-effectiveness and cost-utility analysis of a workplace smoking cessation intervention with and without financial incentives

CHAPTER 10 Implementation of financial incentives for successful smoking cessation in real-life company settings: a qualitative needs assessment among employers

CHAPTER 11 General Discussion

Valorization

Summary

Samenvatting

Dankwoord

Publications 


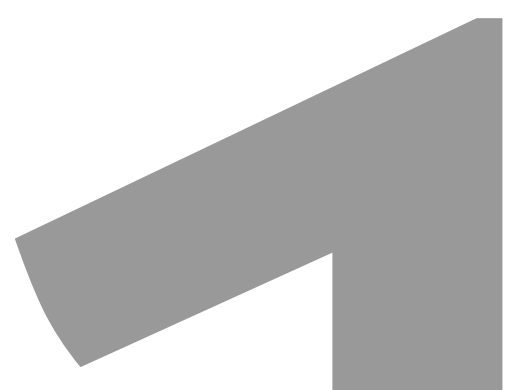

CHAPTER 1 
General introduction 



\section{Socioeconomic inequalities in smoking prevalence}

Tobacco use is the main preventable cause of disease and death worldwide. ${ }^{1}$ Smoking causes cancer, chronic obstructive pulmonary disease (COPD), coronary heart disease, stroke and heart failure. ${ }^{1}$ The life expectancy for smokers is at least 10 years shorter than for nonsmokers. ${ }^{1,2}$ Not only is smoking a major health threat, but also a large cost burden. The global healthcare expenditure due to diseases caused by smoking was estimated to be $€ 325$ billion a year in 2012 , or $5.7 \%$ of global health expenditure. ${ }^{3}$ In Western countries, the proportion of smokers is not equally distributed among low and high socioeconomic groups. ${ }^{4-6}$ Individuals with lower education and income are more likely to smoke and smoke more cigarettes per day than higher educated and more affluent people., ${ }^{7,8}$ This disparity causes smoking to be the single largest contributor to socioeconomic differences in health and mortality observed in Western countries. ${ }^{9,10}$

\section{Smoking in the Netherlands}

Among the general population in the Netherlands in 2018, 22.4\% of individuals aged 18 years and older smoked on a regular basis. ${ }^{11}$ Also in the Netherlands, large socioeconomic differences in smoking are present: $26.9 \%$ of lower educated people smoke compared to $15.7 \%$ of higher educated people. ${ }^{11}$ Additionally, nine out of ten lower educated smokers are daily smokers, compared to only half of higher educated smokers. Although the overall proportion of people who smoke is generally declining in the Netherlands and other Western countries, inequalities in smoking prevalence according to socioeconomic status (SES) have sustained or increased over time..$^{5,6,12-15}$ To decrease the socioeconomic gap in smoking it is crucial to find cessation treatments that are effective for smokers with a low SES, and to make them available and accessible to this important target group. ${ }^{16}$

\section{Socioeconomic barriers for smoking cessation treatment}

Several types of treatments have shown to be effective in helping people to quit smoking, including (a combination of) behavioral counselling, smoking cessation medications, and nicotine replacement therapy. ${ }^{17-20}$ However, a pressing issue presents itself: most tobacco control interventions show lower effectivity in low socioeconomic groups. ${ }^{16,21,22}$ Individuals with a lower SES try to quit just as often as individuals with a higher SES, but they are less likely to succeed. ${ }^{22,23}$ This lower success rate could be caused by multiple factors including higher nicotine addiction levels, less social support for quitting smoking, a lower motivation to quit smoking, more life stress, a lower degree of self-efficacy, and an increased likelihood of not completing smoking cessation treatment. ${ }^{22,24}$ In addition, a literature review found many barriers for lower SES smokers to receive smoking cessation treatment. ${ }^{25}$ These barriers included the use of ineffective (impersonal) recruitment strategies by professionals, poor availability of treatment, and treatment not being attuned to the needs of lower SES smokers (for example not enough treatment sessions and no strategy in case of relapse). Lastly, an important barrier to receive smoking cessation treatment for lower SES smokers is the unaffordability of treatment related to their financial situation or limited health insurance reimbursement. ${ }^{25}$ 


\section{Workplace health promotion}

Health promotion programs at the workplace encourage a healthy lifestyle in employees, with the goal to achieve a better health, higher productivity, and less absence from work due to sickness. The workplace has been considered a suitable environment for health promotion; it provides easy access to large employee populations, people spend a lot of time at work, and coworkers can be a source of social support. ${ }^{26,27}$ Reviews of workplace health promotion interventions have shown their potential effectiveness in improving health and reducing costs, if they use evidence-based methods and are executed and designed well. ${ }^{28}$ Small positive effects of workplace health promotion interventions were shown on various work-related outcomes and health behaviors, such as self-perceived health, absenteeism, productivity at work and work ability; ${ }^{29}$ nutritional behavior;; ${ }^{26,30}$ physical activity; ${ }^{26,31}$ body mass index; ${ }^{32,33}$ and smoking cessation. ${ }^{20,34}$

\section{Smoking cessation at the workplace}

Workplace situated group trainings are effective in helping people quit smoking..$^{35}$ In a group training program, smokers get together for several sessions and collectively quit smoking under the guidance of a stop-smoking coach or health professional. The trainings contain several elements that can contribute to quit success, including teaching knowledge and skills, and increasing motivation. ${ }^{35,36}$

A training at the workplace is a way to make smoking cessation treatment more accessible for employed lower SES smokers. For people who have been considering quitting smoking, the possibility to participate in a smoking cessation program at the workplace can be just the nudge towards a quit attempt. A training during working hours may be perceived as convenient and might lower the threshold to sign up. Other advantages of the workplace as a setting for a smoking cessation intervention are that a large part of the population can be reached, that it may lead to higher participation rates than in a general population, and that by selecting certain companies lower SES smokers can be targeted. ${ }^{20,27}$

Trainings in a group setting may have an advantage over individual counselling because they facilitate social processes such as sharing experiences, peer pressure, and social learning. ${ }^{35}$ These social processes may be an important contributor to successful smoking cessation. ${ }^{37-40}$ Similar to the home environment, in the workplace coworkers can influence each other's smoking and cessation behavior. ${ }^{41,42}$ On the one hand, employees who are exposed to many smoking colleagues may be less likely to attempt to quit smoking. ${ }^{42}$ On the other hand, a network analysis among smokers showed that the likelihood that an employee quit smoking increased by $34 \%$ if a colleague quit smoking. ${ }^{37}$ This shows that employees could influence the health behavior of the people they interact with at work, which can be used to stimulate smoking cessation at the workplace. An additional advantage of quitting smoking in a workplace setting is that it may allow employees to receive social support from fellow quitters during the workday, and that it can create peer pressure to remain abstinent. 


\section{Financial incentives to increase smoking cessation}

Although incentivizing people to adopt healthy behavior has been a known strategy for many decades, there were very few high quality randomized controlled trials on financial incentives for smoking cessation ${ }^{43}$ at the start of this research project. Most studies had been conducted in the United States, only a few in Europe and the rest of the world, and none had been performed in the Netherlands. ${ }^{43,44}$ Reviews at the time indicated that financial incentives could have a moderate positive effect on smoking cessation, 43,44 but the evidence was uncertain because most studies lacked adequate randomization procedures, as well as clear reporting of outcomes, and were at risk for bias. ${ }^{43}$ Moreover, most studies did not combine financial incentives with smoking cessation counseling, while it may be essential to support smokers who are motivated by financial incentives with evidence based behavioral therapy to increase their likelihood of successful smoking cessation.

\section{Indirect effects of financial incentives on smoking cessation}

Why could financial incentives contribute to smoking cessation success? An interesting finding is that simply asking smokers this question may not be sufficient to find the complete answer. Although financial incentives seem effective in randomized controlled trials, ${ }^{45}$ when asked, people do not always state financial incentives to be a motivator to quit smoking, nor do they attribute their quit success to the incentives. ${ }^{46,47}$ The effect of financial incentives on quit success may be mediated by the psychosocial mediators attitudes, social influences and self-efficacy. These mediators can predict a person's intention to change their behaviour, which can lead to actual behavioural change such as quitting smoking. ${ }^{48-51}$ Participants may like the idea of being able to earn rewards for quitting smoking and enjoy receiving the rewards, ${ }^{46}$ which may contribute to a positive attitude toward quitting smoking. Providing financial incentives for quitting smoking may propagate a social norm of non-smoking, ${ }^{50}$ and smokers may receive more encouragement from people in their social environment to reach their goal of quitting smoking and receiving the reward, for example when a spouse also profits from the reward.$^{52}$ Financial incentives may also stimulate smokers to visualize quitting smoking and receiving their reward, and this visualization may consequently increase their self-efficacy. ${ }^{53}$ Finally, financial incentives might motivate smokers to improve their chance of quit success by using smoking cessation medications or nicotine replacement therapy, which enhances smoking cessation success. . $^{18,19}$

\section{Facilitators and barriers for the implementation of financial incentives}

Demonstrating effectiveness of smoking cessation trainings with financial incentives in an experimental setting all but guarantees real-world implementation by companies. To facilitate a successful implementation, it is important to investigate existing barriers that may hinder the implementation, and develop strategies to overcome these ${ }^{54,55}$ Scientific evidence about effective implementation strategies for health promoting activities in 
the workplace setting is scarce and inconsistent. ${ }^{56}$ According to employers, in general the major obstacles for the implementation of employee health programs are a lack of financial and staff resources, insufficient evidence on which programs work best and their return on investment, and a lack of employee interest or low participation in the program. ${ }^{57}$ Of these three, the lack of financial resources is particularly relevant for the decision to implement financial incentives because of the additional investment required on top of the costs of the smoking cessation group training program. A better insight in the cost-effectiveness of financial incentives may therefore contribute to the willingness to implement financial incentives.

Another barrier specific to the implementation of financial incentives for smoking cessation is that rewarding what is considered a 'bad behavior' can be controversial and regarded unfair to employees who do not smoke and cannot earn the incentive. ${ }^{58-60}$ Unlike in the United States, where most of the larger companies offer their employees financial incentives for health promoting behaviors, in Europe, incentivizing employees for health behaviors is still uncommon. ${ }^{57}$ This may be the result of differences in health insurance systems but also in cultural ideas of whether financially incentivizing healthy behavior is acceptable, and whether employers should take an active role in encouraging their employees to take up a healthy lifestyle. ${ }^{57}$

\section{Aim and outline of the dissertation}

Summarizing the above, effective interventions that offer quit success for low-SES smokers are vital to reduce smoking prevalence and to decrease the socioeconomic gap in smoking.

The aim of the current dissertation is to research the effectivity of financial incentives for quit success in combination with a group training program in a workplace setting. The corresponding research questions are the following:

1. Can providing free smoking cessation treatment increase use of treatment, quit attempts and smoking cessation in people who smoke?

2. Are financial incentives for quit success on top of a workplace smoking cessation group training program effective at increasing smoking cessation in employees who smoke?

3. How does a workplace smoking cessation group training program with incentives increase quit success?

4. Are financial incentives on top of a workplace smoking cessation group training program a cost-effective intervention?

5. According to employers, what are the barriers and facilitators for the implementation of a smoking cessation group training program with incentives in workplaces? 
Below, an overview is provided of the chapters in this dissertation, which together contribute to this overall aim.

We aim to answer the first research question in Chapters 2 and 3. Chapter 2, we perform a systematic review that is aimed at assessing the impact of reducing the costs for tobacco smokers or healthcare providers for using or providing smoking cessation treatment on use of smoking cessation treatment, quit attempts, and smoking cessation. Chapter 3 describes a survey study on the relation between lower cost medication and quit attempts and smoking cessation.

The second research question on the effectivity of financial incentives on top of a group training is answered in Chapters 4 and 5. In Chapter 4 we describe the study protocol of a cluster randomized controlled trial on the effect of a workplace-based group training program combined with financial incentives on smoking cessation. Chapter 5 presents the results of this cluster randomized controlled trial.

To answer the third research question, we use quantitative and qualitative methods. In Chapter 6, we conduct qualitative interviews with employees who participated in the study on facilitating factors to quit smoking. In Chapter 7, a questionnaire study is presented to research the association of quit success with peer smoking behavior and social support for quitting. In order to investigate how receiving financial incentives for smoking cessation leads to smoking cessation, we conducted a path-analysis study and tested several mediators of that relationship. These results are presented in Chapter 8.

Chapter 9 assesses the fourth research question regarding the cost-effectiveness of financial incentives. Results are presented of a trial-based economic evaluation to quantify the cost-effectiveness and cost-utility of a workplace smoking cessation intervention with and without financial incentives.

Chapter 10 aims to answer the final research question on the implementation of the intervention. It comprises a study using qualitative interviews with employers aimed at assessing the barriers and facilitators for the implementation of group trainings with financial incentives.

This dissertation concludes with a general discussion in Chapter 11, where the results of all the chapters are discussed and compared to existing literature. Moreover, we deliberate methodological considerations and present our vision on priorities for future research and policy recommendations. 


\section{REFERENCES}

1. US Department of Health and Human Services. The health consequences of smoking-50 years of progress. In. a report of the Surgeon General. Vol 17. Atlanta, GA: US Department of Health and Human Services, Centers for Disease Control and Prevention, National Center for Chronic Disease Prevention and Health Promotion, Office on Smoking and Health; 2014.

2. Jha P, Ramasundarahettige $C$, Landsman V, et al. 21st-Century Hazards of Smoking and Benefits of Cessation in the United States. N Engl J Med. 2013;368(4):341-350.

3. Goodchild M, Nargis N, Tursan d'Espaignet E. Global economic cost of smoking-attributable diseases. Tob Control. 2018;27(1):58-64.

4. Reid JL, Hammond D, Boudreau C, Fong GT, Siahpush M. Socioeconomic disparities in quit intentions, quit attempts, and smoking abstinence among smokers in four western countries: findings from the International Tobacco Control Four Country Survey. Nicotine \& tobacco research : official journal of the Society for Research on Nicotine and Tobacco. 2010;12 SuppI:S20-33.

5. Hiscock R, Bauld L, Amos A, Platt S. Smoking and socioeconomic status in England: the rise of the never smoker and the disadvantaged smoker. J Public Health (Oxf). 2012;34(3):390-396.

6. Nagelhout GE, de Korte-de Boer $D$, Kunst $A E$, et al. Trends in socioeconomic inequalities in smoking prevalence, consumption, initiation, and cessation between 2001 and 2008 in the Netherlands. Findings from a national population survey. BMC Public Health. 2012;12(1):1-9.

7. Huisman M, Kunst AE, Mackenbach JP. Inequalities in the prevalence of smoking in the European Union: comparing education and income. Prev Med. 2005;40(6):756-764.

8. Schaap MM, Kunst AE. Monitoring of socio-economic inequalities in smoking: Learning from the experiences of recent scientific studies. Public Health. 2009;123(2):103-109.

9. Siahpush M, English D, Powles J. The contribution of smoking to socioeconomic differentials in mortality: results from the Melbourne Collaborative Cohort Study, Australia. J Epidemiol Community Health. 2006;60(12):1077-1079.

10. Jha P, Peto R, Zatonski W, Boreham J, Jarvis MJ, Lopez AD. Social inequalities in male mortality, and in male mortality from smoking: indirect estimation from national death rates in England and Wales, Poland, and North America. The Lancet. 2006;368(9533):367-370.

11. Bommelé J, Willemsen M. Kerncijfers roken 2018 [Key figures smoking 2018]. Utrecht2020.

12. Giskes K, Kunst AE, Benach J, et al. Trends in smoking behaviour between 1985 and 2000 in nine European countries by education. J Epidemiol Community Health. 2005;59(5):395-401.

13. Gielkens-Sijstermans CM, Mommers MA, Hoogenveen RT, et al. Reduction of smoking in Dutch adolescents over the past decade and its health gains: a repeated cross-sectional study. Eur J Public Health. 2010;20(2):146-150.

14. Harper S, Lynch J. Trends in socioeconomic inequalities in adult health behaviors among US states, 1990-2004. Public Health Rep. 2007;122(2):177-189.

15. Federico B, Costa G, Kunst AE. Educational inequalities in initiation, cessation, and prevalence of smoking among 3 Italian birth cohorts. Am J Public Health. 2007;97(5):838-845.

16. Brown T, Platt $S$, Amos A. Equity impact of European individual-level smoking cessation interventions to reduce smoking in adults: a systematic review. EurJPublic Health. 2014;24(4):551556.

17. Hartmann-Boyce J, Stead LF, Cahill K, Lancaster T. Efficacy of interventions to combat tobacco addiction: Cochrane update of 2012 reviews. Addiction. 2013;108.

18. Cahill K, Stevens S, Perera R, Lancaster T. Pharmacological interventions for smoking cessation: an overview and network meta-analysis. Cochrane Database Syst Rev. 2013(5):CD009329. 
19. Stead LF, Koilpillai P, Fanshawe TR, Lancaster T. Combined pharmacotherapy and behavioural interventions for smoking cessation. Cochrane Database of Systematic Reviews. 2016(3).

20. Cahill K, Lancaster T. Workplace interventions for smoking cessation. The Cochrane Library. 2014.

21. Hill S, Amos A, Clifford D, Platt S. Impact of tobacco control interventions on socioeconomic inequalities in smoking: review of the evidence. Tob Control. 2014;23(e2):e89-e97.

22. Hiscock R, Bauld L, Amos A, Fidler JA, Munafò M. Socioeconomic status and smoking: a review. Ann N Y Acad Sci. 2012;1248.

23. Kotz D, West R. Explaining the social gradient in smoking cessation: it's not in the trying, but in the succeeding. Tob Control. 2009;18(1):43-46.

24. Burns EK, Levinson AH. Discontinuation of nicotine replacement therapy among smokingcessation attempters. Am J Prev Med. 2008;34(3):212-215.

25. van Wijk EC, Landais LL, Harting J. Understanding the multitude of barriers that prevent smokers in lower socioeconomic groups from accessing smoking cessation support: A literature review. Prev Med. 2019;123:143-151.

26. Hutchinson $A D$, Wilson $C$. Improving nutrition and physical activity in the workplace: a metaanalysis of intervention studies. Health Promot Int. 2011;27(2):238-249.

27. Fishwick D, Carroll C, McGregor M, et al. Smoking cessation in the workplace. Occup Med (Lond). 2013;63(8):526-536.

28. Goetzel RZ, Henke RM, Tabrizi M, et al. Do workplace health promotion (wellness) programs work? J Occup Environ Med. 2014;56(9):927-934.

29. Rongen A, Robroek SJW, van Lenthe FJ, Burdorf A. Workplace Health Promotion: A Meta-Analysis of Effectiveness. Am J Prev Med. 2013;44(4):406-415.

30. Maes L, Van Cauwenberghe E, Van Lippevelde W, et al. Effectiveness of workplace interventions in Europe promoting healthy eating: a systematic review. Eur J Public Health. 2011;22(5):677-683.

31. Conn VS, Hafdahl AR, Cooper PS, Brown LM, Lusk SL. Meta-Analysis of Workplace Physical Activity Interventions. Am J Prev Med. 2009;37(4):330-339.

32. Mulchandani R, Chandrasekaran AM, Shivashankar R, et al. Effect of workplace physical activity interventions on the cardio-metabolic health of working adults: systematic review and metaanalysis. Int J Behav Nutr Phys Act. 2019;16(1):134-134.

33. Anderson LM, Quinn TA, Glanz K, et al. The Effectiveness of Worksite Nutrition and Physical Activity Interventions for Controlling Employee Overweight and Obesity: A Systematic Review. Am J Prev Med. 2009;37(4):340-357.

34. Fichtenberg CM, Glantz SA. Effect of smoke-free workplaces on smoking behaviour: systematic review. BMJ (Clinical research ed). 2002;325(7357):188-188.

35. Stead LF, Carroll AJ, Lancaster T. Group behaviour therapy programmes for smoking cessation. Cochrane Database Syst Rev. 2017(3).

36. Kotsen C, Santorelli ML, Bloom EL, et al. A Narrative Review of Intensive Group Tobacco Treatment: Clinical, Research, and U.S. Policy Recommendations. Nicotine \& Tobacco Research. 2018:nty162-nty162.

37. Christakis NA, Fowler JH. The collective dynamics of smoking in a large social network. N Engl J Med. 2008;358(21):2249-2258.

38. Hitchman SC, Fong GT, Zanna MP, Thrasher JF, Laux FL. The relation between number of smoking friends, and quit intentions, attempts, and success: findings from the International Tobacco Control (ITC) Four Country Survey. Psychol Addict Behav. 2014;28(4):1144-1152. 
39. Holahan CK, Holahan CJ, Li X, Jung SJ. Social influences on smoking in American workers: the role of the presence of smokers in the workplace and in the home. Am J Health Promot. 2013;28(2):105-107.

40. Christakis NA, Fowler JH. The Collective Dynamics of Smoking in a Large Social Network. NEngl J Med. 2008;358(21):2249-2258.

41. Quist HG, Christensen U, Carneiro IG, Hansen JV, Bjorner JB. Do colleagues influence our lifestyle: the matter of smoking, body mass index and leisure-time physical activity? Prev Med. 2014;67:166-170.

42. Yong LC, Luckhaupt SE, Li J, Calvert GM. Quit interest, quit attempt and recent cigarette smoking cessation in the US working population, 2010. Occup Environ Med. 2014;71(6):405-414.

43. Cahill K, Hartmann-Boyce J, Perera R. Incentives for smoking cessation. The Cochrane database of systematic reviews. 2015(5):CD004307.

44. Giles EL, Robalino S, McColl E, Sniehotta FF, Adams J. The Effectiveness of Financial Incentives for Health Behaviour Change: Systematic Review and Meta-Analysis. PLoS One. 2014;9(3):e90347.

45. Volpp KG, Troxel AB, Pauly MV, et al. A randomized, controlled trial of financial incentives for smoking cessation. N Engl J Med. 2009;360(7):699-709.

46. Kim A, Kamyab K, Zhu J, Volpp K. Why are Financial Incentives not Effective at Influencing Some Smokers to Quit? Results of a Process Evaluation of a Worksite Trial Assessing the Efficacy of Financial Incentives for Smoking Cessation. J Occup Environ Med. 2011;53(1):62-67.

47. Ormston R, van der Pol M, Ludbrook A, McConville S, Amos A. quit4u: the effectiveness of combining behavioural support, pharmacotherapy and financial incentives to support smoking cessation. Health Educ Res. 2015;30(1):121-133.

48. Vries HD, Mudde AN. Predicting stage transitions for smoking cessation applying the attitudesocial influence-efficacy model. Psychol Health. 1998;13(2):369-385.

49. Ajzen I. The theory of planned behavior. Organ Behav Hum Decis Process. 1991;50(2):179-211.

50. van den Putte B, Yzer MC, Brunsting S. Social influences on smoking cessation: a comparison of the effect of six social influence variables. Prev Med. 2005;41(1):186-193.

51. de Vries H, Mudde A, Leijs I, et al. The European Smoking Prevention Framework Approach (EFSA): an example of integral prevention. Health Educ Res. 2003;18(5):611-626.

52. Donatelle RJ, Prows SL, Champeau D, Hudson D. Randomised controlled trial using social support and financial incentives for high risk pregnant smokers: Significant Other Supporter (SOS) program. Tob Control. 2000;9.

53. Bandura A. Self-efficacy: toward a unifying theory of behavioral change. Psychol Rev. 1977;84(2):191-215.

54. Glasgow RE, Vogt TM, Boles SM. Evaluating the public health impact of health promotion interventions: the RE-AIM framework. Am J Public Health. 1999;89(9):1322-1327.

55. Hulscher M, Wensing M, Grol R. Multifaceted strategies for improvement. In: Grol R, Wensing $\mathrm{M}$, Eccles M, Davis D, eds. Improving patient care: the implementation of change in health care. Chichester, UK: John Wiley \& Sons, Ltd.; 2013:278-287.

56. Wolfenden L, Goldman S, Stacey FG, et al. Strategies to improve the implementation of workplace-based policies or practices targeting tobacco, alcohol, diet, physical activity and obesity. Cochrane Database of Systematic Reviews. 2018(11).

57. Willis Towers Watson. Employee Health and Business Success. Making the connections and taking action. Global research summary. 2016. 
58. Giles EL, Robalino S, Sniehotta FF, Adams J, McColl E. Acceptability of financial incentives for encouraging uptake of healthy behaviours: A critical review using systematic methods. Prev Med. 2015;73:145-158.

59. Halpern SD, Madison KM, Volpp KG. Patients as mercenaries?: the ethics of using financial incentives in the war on unhealthy behaviors. Circ Cardiovasc Qual Outcomes. 2009;2(5):514516.

60. Giles EL, Sniehotta FF, McColl E, Adams J. Acceptability of financial incentives for health behaviour change to public health policymakers: a qualitative study. BMC Public Health. 2016;16(1):989. 


\section{CHAPTER 2}




\section{Healthcare financing systems for increasing the use of tobacco dependence treatment}

Van den Brand FA, Nagelhout GE, Reda AA, et al. Healthcare financing systems for increasing the use of tobacco dependence treatment. 


\section{ABSTRACT}

Background Tobacco smoking is the leading preventable cause of death worldwide, which makes it essential to stimulate smoking cessation. The financial cost of smoking cessation treatment can act as a barrier to those seeking support. We hypothesized that provision of financial assistance for people trying to quit smoking, or reimbursement of their care providers, could lead to an increased rate of successful quit attempts. This is an update of the original 2005 review.

Objectives The primary objective of this review was to assess the impact of reducing the costs for tobacco smokers or healthcare providers for using or providing smoking cessation treatment through healthcare financing interventions on abstinence from smoking. The secondary objectives were to examine the effects of different levels of financial support on the use or prescription of smoking cessation treatment, or both, and on the number of smokers making a quit attempt (quitting smoking for at least 24 hours). We also assessed the cost effectiveness of different financial interventions, and analyzed the costs per additional quitter, or per quality-adjusted life year (QALY) gained.

Search methods We searched the Cochrane Tobacco Addiction Group Specialized Register in September 2016.

Selection criteria We considered randomized controlled trials (RCTs), controlled trials and interrupted time series studies involving financial benefit interventions to smokers or their healthcare providers, or both.

Data collection and analysis Two reviewers independently extracted data and assessed the quality of the included studies. We calculated risk ratios (RR) for individual studies on an intention-to-treat basis and performed meta-analysis using a random-effects model.

Main results In the current update, we have added six new relevant studies, resulting in a total of 17 studies included in this review involving financial interventions directed at smokers or healthcare providers, or both. Full financial interventions directed at smokers had a favorable effect on abstinence at six months or longer when compared to no intervention (RR $1.77,95 \% \mathrm{Cl} 1.37$ to $2.28,10=33 \%, 9333$ participants). There was no evidence that full coverage interventions increased smoking abstinence compared to partial coverage interventions (RR $1.02,95 \% \mathrm{Cl} 0.71$ to $1.48, \mathrm{IO}=64 \%, 5914$ participants), but partial coverage interventions were more effective in increasing abstinence than no intervention (RR $1.2795 \% \mathrm{Cl} 1.02$ to $1.59, \mathrm{IO}=21 \%, 7108$ participants). The economic evaluation showed costs per additional quitter ranging from USD 97 to USD 7646 for the comparison of full coverage with partial or no coverage. There was no clear evidence of an effect on smoking cessation when we pooled two trials of financial incentives directed at healthcare providers ( $\mathrm{RR} 1.16, \mathrm{Cl} 0.98$ to $1.37, \mathrm{IO}=0 \%, 2311$ participants). Full financial interventions increased the number of participants making a quit attempt when compared to no interventions ( $\mathrm{RR} 1.11,95 \% \mathrm{Cl} 1.04$ to $1.17, \mathrm{IO}=15 \%, 9065$ participants). There was insufficient evidence to show whether partial financial interventions increased quit attempts compared to no interventions (RR $1.13,95 \% \mathrm{Cl} 0.98$ to $1.31, \mathrm{IO}=88 \%, 6944$ 
participants). Full financial interventions increased the use of smoking cessation treatment compared to no interventions with regard to various pharmacological and behavioral treatments: nicotine replacement therapy (NRT): RR 1.79, 95\% Cl 1.54 to $2.09,10=35 \%$, 9455 participants; bupropion: RR 3.22, 95\% Cl 1.41 to 7.34 , IO = 71\%, 6321 participants; behavioral therapy: RR $1.77,95 \% \mathrm{Cl} 1.19$ to 2.65 , IO $=75 \%, 9215$ participants. There was evidence that partial coverage compared to no coverage reported a small positive effect on the use of bupropion (RR $1.15,95 \% \mathrm{Cl} 1.03$ to $1.29, \mathrm{IO}=0 \%, 6765$ participants). Interventions directed at healthcare providers increased the use of behavioral therapy (RR 1.69, 95\% Cl 1.01 to $2.86, \mathrm{IO}=85 \%, 25820$ participants), but not the use of NRT and/ or bupropion (RR $0.94,95 \% \mathrm{Cl} 0.76$ to $1.18, \mathrm{IO}=6 \%, 2311$ participants). We assessed the quality of the evidence for the main outcome, abstinence from smoking, as moderate. In most studies participants were not blinded to the different study arms and researchers were not blinded to the allocated interventions. Furthermore, there was not always sufficient information on attrition rates. We detected some imprecision, but we judged this to be of minor consequence on the outcomes of this study.

Conclusions Full financial interventions directed at smokers when compared to no financial interventions increase the proportion of smokers who attempt to quit, use smoking cessation treatments, and succeed in quitting. There was no clear and consistent evidence of an effect on smoking cessation from financial incentives directed at healthcare providers. We are only moderately confident in the effect estimate because there was some risk of bias due to a lack of blinding in participants and researchers, and insufficient information on attrition rates. 


\section{BACKGROUND}

Tobacco smoking is a major risk factor for non-communicable diseases. A range of interventions such as pharmacotherapy and counselling are currently in use to treat nicotine dependence. ${ }^{1}$ Although the majority of smokers attempt to quit unassisted, ${ }^{2}$ treatments are recommended, as they can increase quit success. ${ }^{1,3-6}$ Existing management strategies for smoking cessation remain limited in effectiveness since a significant majority of smokers attempting to quit either fail to quit or relapse after a successful quit..$^{7-9} \mathrm{Apart}$ from counselling and pharmacotherapy, strategies such as financial interventions may be useful to address the problem in a multifaceted manner and increase cessation rates. ${ }^{10,11}$ Regardless of scientific acceptability, treatment utilization by smokers and selection of treatment strategies by healthcare providers and insurance companies are influenced by cost considerations, as documented in non-experimental studies. ${ }^{12-16} \mathrm{As}$ a result, costs and a variety of financial interventions are becoming increasingly important considerations in medical practice to improve patient outcomes and quality of service. ${ }^{17-19}$ For instance, an international survey reported that in several countries a larger proportion of physicians are likely to receive financial incentives based on quality improvement targets: the percentage of physicians provided with incentives was $89 \%$ in the UK; $81 \%$ in the Netherlands; $70 \%$ in Italy; and 65\% in Austria. ${ }^{20}$ Despite their popularity, financial interventions are shown to have at best weak or mixed impacts on service quality and patient outcomes.18,21-24 In a review of 128 studies that implemented financial incentives in healthcare, Van Herck and colleagues indicated that, for incentive schemes to be effective, they need to be applied to individuals or teams and have prior set targets for quality and patient outcome improvements. ${ }^{19}$ Reviews also indicate that there is little or no information on the cost-effectiveness of these incentives. ${ }^{19,21,25}$ According to reports, smoking cessation treatments are highly cost effective compared to other, commonly implemented preventive interventions, such as hypertension and cholesterol-lowering treatments. ${ }^{17,26}$ In a systematic review of the literature, Ronckers and colleagues reported that despite lack of standardized reporting by the included studies, smoking cessation treatments aimed at reducing smoking prevalence are cost-effective. ${ }^{27}$ Considering limited resources and the increasing cost of health care, it is pertinent to examine the impact of financial interventions provided to smokers and healthcare providers on treatment and process related outcomes such as smoking cessation service use. We hypothesized that provision of financial assistance for smokers trying to quit, or reimbursement of their healthcare providers, could lead to an increased rate of quit attempts, utilization of smoking cessation treatments, and successful quitting. This review evaluates financial interventions directed at individual smokers regardless of whether they achieve cessation and evaluates financial interventions directed at healthcare providers. A separate Cochrane Review evaluates competitions and incentives to reward smokers who achieve cessation or abstinence in smoking cessation schemes. ${ }^{28}$

\section{OBJECTIVES}

The primary objective of this review was to assess the impact of reducing the costs of providing or using smoking cessation treatment by healthcare financing interventions 
on abstinence from smoking. The secondary objectives were to examine the effects of different levels of financial support on the use or prescription of smoking cessation treatment, or both, and on the number of smokers making a quit attempt (quitting smoking for at least 24 hours). We also assessed the cost effectiveness of different financial interventions, and analyzed the costs per additional quitter, or per quality-adjusted life year (QALY) gained.

\section{METHODS}

\section{Criteria for considering studies for this review}

\section{Types of studies}

We considered randomized controlled trials (RCTs), controlled trials (CTs) and interrupted time series (ITS) studies. We included ITS studies if they had at least three time points before and three after the intervention, and if they had a clearly defined intervention point.

\section{Types of participants}

We included those studies in which the study population consisted of smokers or healthcare providers. We assessed the primary and secondary objectives from either a smoker's or a healthcare provider's perspective. For smokers, the aim of the healthcare financing interventions had to be to encourage the use of smoking cessation treatment and successful quit attempts. When the intervention was directed towards healthcare providers, the intervention had to aim to affect the prescribing of smoking cessation treatment or the smoking behavior of the participants, or both, by offering assistance to quit smoking.

\section{Types of interventions}

We included trials that studied the effects of healthcare financing interventions directed at smokers or healthcare providers for increasing the use of smoking cessation treatment (e.g. delivered by government, healthcare insurance plans, or other institution-arranged interventions).

We classified patient-centered financial interventions as:

- Health insurance coverage - changes to the level of benefit available for smoking cessation treatments, including changes to copayment or out-of-pocket payments made by people receiving treatment.

- Direct coverage-changes to the direct cost to the smoker of using smoking cessation treatment, for example by provision of a prescription for free pharmacotherapy.

- Health insurance cost - changes to the premiums or user fees paid for health insurance. 
We defined healthcare provider-centered financing interventions as:

- Salary - payment for a set number of working hours or sessions per time unit.

- Capitation - a set amount of payment per patient for providing specific care.

- Fee-for-service - payment for every item of service or unit of care provided.

- Target payment (pay for performance, P4P) - payment only made with respect to achievement of an agreed target.

- Fund holding- and organization-level payment systems - which can improve the working conditions within an organization and can indirectly influence the salary of a healthcare provider.

We differentiated between healthcare financing interventions for individual smokers and for healthcare providers. In patients, for example, comparisons can be made between full financial coverage and partial financial coverage. For healthcare providers, a maximum target payment can be compared with no target payment. There were no restrictions on the type of smoking cessation treatment for which the financial benefit could be offered. This could include pharmacotherapy (e.g. nicotine replacement therapy, varenicline or bupropion), behavioral support, or a combination thereof. When the financial intervention of a study was aimed at more than one type of smoking cessation treatment, the effect of the financial intervention could be spread out over the different

types of products. Studies of financial interventions that are aimed at more than one type of smoking cessation treatment therefore cannot formally be compared with studies that offer coverage for only one product. This is the reason for differentiating between partial and full interventions as discussed in the next paragraph. As a result, we focused on full and partial financial interventions.

As a smoker could use more than one type of product, summing the use of the different types of smoking cessation treatment could overestimate the number of smokers who used smoking cessation treatment. We classified the patient-directed financial benefits as full, partial or no intervention, based on theoretical and practical considerations. We considered an intervention that covered the cost of both pharmacotherapy and behavioral support to be a full financial intervention. Full financing need not come from the trial. If there were already existing provisions (e.g. insurance) for partial coverage of smoking cessation treatment (pharmacotherapy or behavioral support) and a trial's intervention(s) complemented this benefit by financing either a pharmacotherapy or behavioral support leading to full coverage of smoking cessation treatment, we classified it as a full-coverage intervention. In this case we considered the control group to be partial financial intervention. More specifically, if an intervention provided coverage for either pharmacotherapy or behavioral support, we considered it partial financial coverage. If a study involved gradations of partial financial intervention, we considered the one with more benefits from the trial's perspective to be the intervention group while the group 
receiving lesser benefits was the control group. If, in a given study, a control group received neither trial-based nor already existing financing arrangements for smoking cessation treatment, we considered it a no-financing intervention. On these bases, we employed further stratification depending on the availability of study data.

\section{Types of outcome measures}

We included studies when they used at least one of the following outcome measures to describe the effects of the intervention. The primary outcome measure of this review was:

- abstinence from smoking. We included studies reporting abstinence from smoking at least six months after the start of the intervention, and we used the longest available follow-up as the preferred outcome measure (SRNT 2002; Hughes 2003)..$^{29,30}$ Biochemically validated abstinence was preferred to self-reported abstinence, and continuous or prolonged abstinence was preferred to pointprevalence abstinence.

The secondary outcome measures were:

- number of participants making a quit attempt, defined as the number of participants who attempted to quit at least once. A quit attempt is defined as not having smoked for at least 24 hours.

- Use of smoking cessation treatment, defined as the number of participants who reported having used smoking cessation treatment or who were registered by healthcare providers or medical insurance organizations as having used smoking cessation treatment.

\section{Economic Evaluation}

To evaluate the cost effectiveness of financial interventions for smoking cessation treatment, we considered data from studies that examined both cost and effects and compared two or more alternatives.

The primary outcome measure of the economic evaluation was smoking-related:

- costs per additional quitter.

The secondary outcome measure was:

- costs per quality-adjusted life year saved (QALY). This measure of health outcome incorporates the effect of an intervention on both the length and the quality of life.

\section{Search methods for identification of studies}

We identified eligible studies for the current update by electronic search of the Cochrane Tobacco Addiction Group Specialized Register, limited to records added since April 2012. This Register includes citations identified via highly sensitive searches for potential reports of controlled trials and other evaluations of interventions for smoking cessation 
and prevention. At the time of the search on 1 September 2016, the Register included the results of searches of the Cochrane Central Register of Controlled trials (CENTRAL; 2016, issue 7) in the Cochrane Library; MEDLINE (via OVID), to update 20160729, Embase (via OVID), to update week 201639 and PsycINFO (via OVID) to update 20160725.

The search strategy (Appendix 1) included MeSH and text terms related to health care costs, health insurance coverage, reimbursement, remuneration, incentives, salaries and fees. There was no limitation on language. The Cochrane Tobacco Addition Group's Information Specialist performed the register search, and prescreened retrieved records for relevance to the topic.

In order to retrieve unpublished studies, we contacted experts in the field via a standardized email. We included unpublished studies or abstracts only when sufficient data were available. For the original review and first update, additional searches of MEDLINE (Appendix 2) and Embase were conducted that combined topic-related terms with the same smoking- and design-related terms used in the regular searches for the Register. Since these records are a subset of the records retrieved and screened during regular searches, we judged this no longer necessary.

\section{Data collection and analysis}

\section{Selection of studies}

Based on title, keywords and abstract, three reviewers (FB, GN, AAR) each selected studies independently of each other by applying the inclusion criteria to the studies identified by the literature search. When there was any doubt whether to select a study or not, we resolved it through discussion. Two reviewers (FB and GN) assessed the full-paper versions of the selected studies in duplicate for each study and independently of each other. If disagreements about inclusion were not resolved by consensus, we arranged to consult additional reviewers (DK and AAR).

\section{Data extraction and management}

Two reviewers (FB and GN) separately and independently extracted data from the included studies, after which the extracted data were compared and discussed. Any disagreements between the two reviewers were resolved by discussion. We extracted the following data.

- Methods: setting (location of care, country, and year of study) and study design

- Participants and/or healthcare providers: method of recruitment, inclusion criteria, characteristics of study population (smoking status, age, gender and motivation to quit smoking)

- Interventions: description of the intervention for each group 
- Outcome measures: definition for each study of continuous abstinence or pointprevalence abstinence, number of participants making a quit attempt, prescription and use of smoking cessation treatment

- Results: we extracted the findings of each study for pooling. Two reviewers (AAR and SE) extracted data concerning the economic evaluations that we used to answer our secondary objectives. We resolved any discrepancies by discussion. We extracted the following data.

- Perspective and time horizon of the economic evaluation

- Direct costs: volume and value of costs of the use of smoking cessation treatment, costs of consultations with healthcare providers and overhead costs (no research costs)

- Indirect costs: volume and value of general medical care, lost productivity, time and travel costs spent by participants visiting healthcare providers

- Discounting and sensitivity analyses

- Results of the economic evaluations

\section{Assessment of risk of bias in included studies}

We assessed the risk of bias of the included studies using criteria from Cochrane included in the Review Manager (RevMan) 5 software (Higgins 2011; RevMan 2014). ${ }^{31,32}$ The criteria have three levels (low risk of bias, high risk of bias, unclear risk of bias) and the judgement on each item could be complemented using quotes from the study report and the raters' comments. We rated the following criteria: sequence generation; allocation concealment; blinding of intervention; issues of incomplete data and loss to follow-up; and other unclassified bias identified by the review authors. We summarized findings from these criteria in Figure 1. The reviewers assessing risk of bias were not blinded to the authors, institution or journal title. Two reviewers (FB and GN) independently rated the studies. We held a consensus meeting to discuss and resolve disagreements between the two reviewers. If a study did not contain sufficient information on methodological criteria or the information was unclear, we contacted the study authors for additional information.

\section{Economic Evaluation}

We assessed the quality of the economic evaluations using the Consensus Health Economic Criteria (CHEC) list (Evers 2005). ${ }^{33} \mathrm{As}$ its name indicates, the CHEC list was produced based on expert consensus. It consists of 19 items as listed in Appendix 3 and incorporates the following aspects: clearly described study population (age, gender and educational level), a description of the intervention and the alternatives, a well-defined research question, an economic study design in which the costs and effects of two or more interventions are compared, a time horizon and perspective of the evaluation, the identification of relevant costs and consequences for each alternative, the measurement of costs and consequences, appropriately valued cost and consequences, the performance of an incremental analysis, 
the performance of discounting and sensitivity analysis, the conclusions following from the data reported, the generalizability of results, statement of conflict of interest and appropriate discussion of ethical and distributional issues. SE independently assessed the quality of the economic evaluations. Items scored as 'yes' received one point. Items scored as 'unclear' or 'no' received no points. We calculated a total score by summing the score of the 19 items (range 0 to 19).

\section{Measures of treatment effect}

We used only intention-to-treat analyses. If the study did not present an intention-totreat analysis, then we recalculated the published data on an intention-to-treat basis. We counted all dropouts and participants lost to follow-up as continuing smokers or making no quit attempt or not having used smoking cessation treatment. For each study outcome we calculated the risk ratio (RR) and the corresponding $95 \%$ confidence interval $(\mathrm{Cl})$ as a measure of intervention effect. Since the outcomes (abstinence, quit attempts and use of treatment) were favorable, a ratio greater than unity indicates an outcome favoring financial intervention. Where we calculated pooled effects, we used a Mantel-Haenszel method to estimate the RR using a random-effects model. We considered pooling when at least two trials assessed the effects of healthcare financing interventions and reported data on the same outcome measure. We conducted a formal statistical test for betweenstudies variance, and assessed whether the observed variability in effect sizes was greater than would be expected to occur by chance (sampling error). We used the 12 statistic, given by the formula ((Q-df)/Q)* $100 \%$, to investigate heterogeneity (where $\mathrm{Q}$ represents Cochrane's Chi2 statistic and df is the degree of freedom, Higgins 2003). ${ }^{34}$ We considered a pooled analysis to have moderate and high heterogeneity when the 12 statistic was more than $50 \%$ and $75 \%$ respectively.

\section{Economic Evaluation}

The transferability of cost estimates of different economic evaluations is mostly restricted by differences in setting. These differences can be related to patient characteristics, incidence of smoking-related diseases, availability of health resources, variations in clinical practice, incentives to healthcare providers and relative prices or costs (Drummond 2015). ${ }^{35}$ Pooling of the different economic evaluations is only permissible when there is no interaction between the setting and the effect of the intervention on medical consumption (Drummond 2015)..$^{35}$ Where pooling was allowed, the volumes of medical consumption, like the use of smoking cessation treatment, were to be pooled and multiplied with the pooled costs per unit consumption. The total costs were calculated in US dollars (USD). When the cost estimates of the different economic evaluations were not transferable, we presented cost data of the individual studies. When no incremental ratios were presented, we calculated the incremental cost effectiveness ratios ourselves. First, we calculated the total costs per group. We then divided the difference in costs between the groups by the difference in number of quitters between the groups. The authors of the studies involved checked the calculation. When currencies other than USD were involved, we used the exchange rates provided by study authors. 


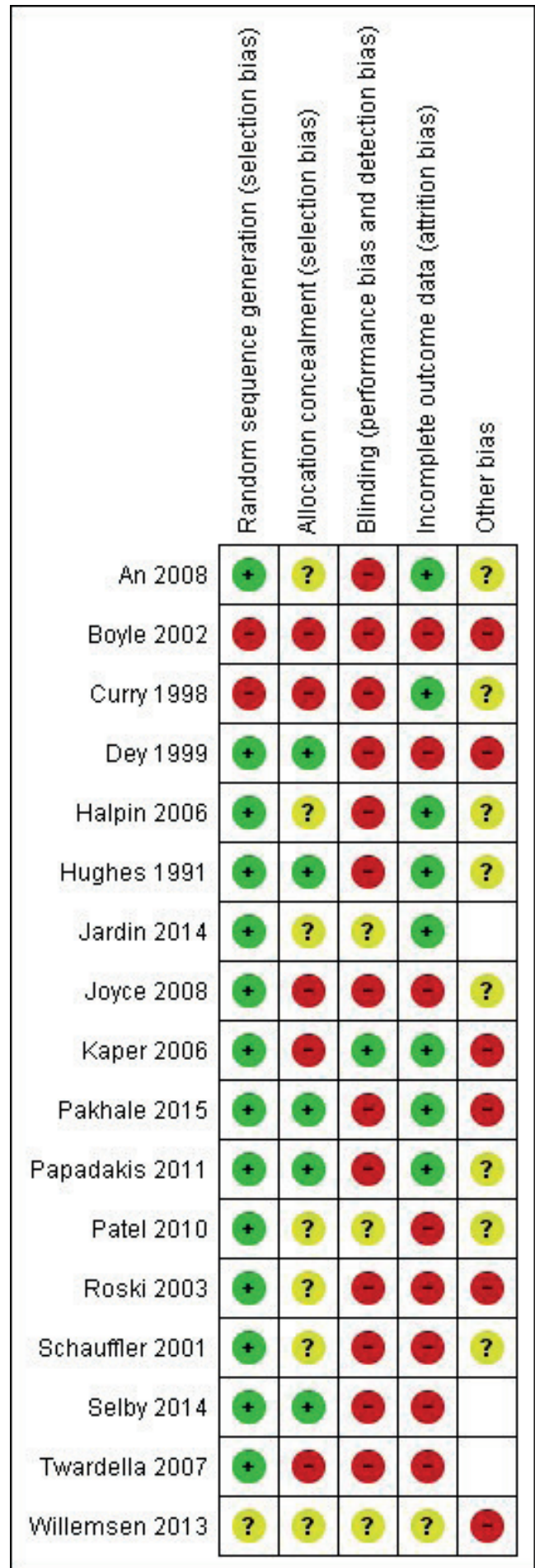

Figure 1. Risk of bias summary: review authors' judgements about each risk of bias item for each included study 


\section{'Summary of findings' table}

Following standard Cochrane methodology (Schünemann 2011), ${ }^{36}$ we created 'Summary of findings' tables for our primary outcome for interventions directed at smokers (Summary of findings for the main comparison) and interventions directed at healthcare providers (Summary of findings 2). This included a GRADE evaluation of the quality of evidence, based on the five standard considerations (study limitations, consistency of effect, imprecision, indirectness and publication bias) (Guyatt 2011). ${ }^{37}$

\section{RESULTS}

\section{Description of studies}

\section{Results of the search}

We identified 3518 references using the search strategy described above, of which 28 were identified from the Register search for this update (for a PRISMA flow chart see Figure 2) (Moher 2009). ${ }^{38}$ After we had removed duplicates, 23 studies remained, of which six new studies were included in the current update (Patel 2010; Papadakis 2011; Willemsen 2013,

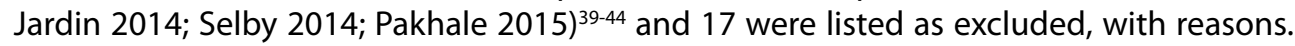
With the six new studies added in this update, we included a total of 17 studies in this review.

\section{Included studies}

The main features of the 17 included studies are described below.

\section{Setting and design}

Ten of the included studies were performed in the USA (Hughes 1991; Curry 1998; Schauffler 2001; Boyle 2002; Roski 2003; Halpin 2006; An 2008; Joyce 2008; Patel 2010; Jardin 2014). ${ }^{39,42,45-52}$ The others were conducted in Canada (Papadakis 2011; Selby 2014; Pakhale 2015), ${ }^{40,41,43}$ the UK (Dey 1999), ${ }^{53}$ the Netherlands (Kaper 2006; Willemsen 2013) ${ }^{44,54}$ and Germany (Twardella 2007). ${ }^{55}$ Four studies were conducted in co-operation with health insurance organizations (Curry 1998; Schauffler 2001; Boyle 2002; Kaper 2006). ${ }^{46,47,52,56}$ Two studies were conducted in family practices (Hughes 1991; Dey 1999), ${ }^{49,53}$ two in an Ottawa hospital clinic (stroke prevention: Papadakis 2011, respirology: Pakhale 2015) ${ }^{40,41}$ and one study was conducted in 40 clinics of a multi-speciality medical group practice (Roski 2003). ${ }^{51}$ Of the seventeen included studies, eleven randomly assigned the individual participants to the treatment group and one or two control groups (Hughes 1991; Dey 1999; Schauffler 2001; Roski 2003; Halpin 2006; Kaper 2006; Patel 2010; Papadakis 2011; Jardin 2014; Selby 2014; Pakhale 2015) $39-43,48,49,51-53,56$ and three randomly assigned medical practices (Twardella 2007; An 2008; Joyce 2008). ${ }^{45,50,55}$ Two studies (Curry 1998; Boyle 2002) ${ }^{46,47}$ were controlled natural experiments with two and four different benefit groups, respectively. One study used a descriptive time-series analysis (Willemsen 2013). ${ }^{44}$ 


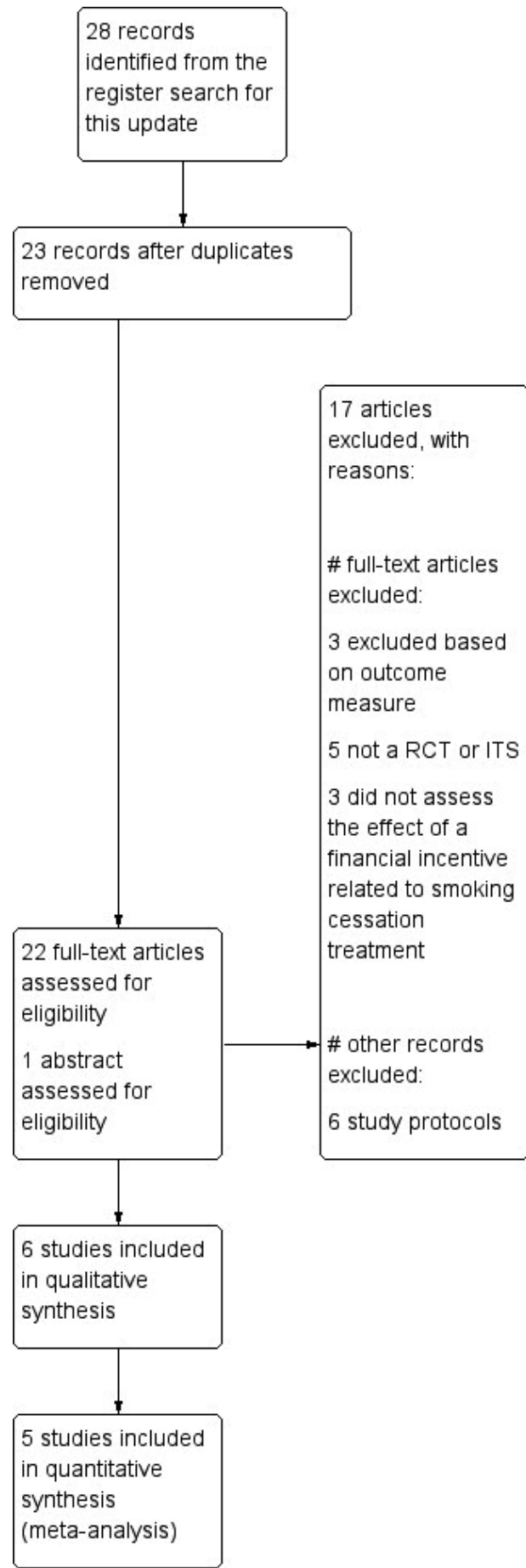

Figure 2. Prisma study flow diagram of studies included in most recent update of this review 


\section{Participants}

Fifteen studies were directed at individuals (Hughes 1991; Curry 1998; Dey 1999; Schauffler 2001; Boyle 2002; Halpin 2006; Kaper2006; Twardella 2007; Joyce 2008; Patel 2010; Papadakis 2011; Jardin 2014; Selby 2014; Willemsen 2013; Pakhale 2015). ${ }^{39-44,46-50,52-55}$ Sample sizes of the included studies varied from 28 participants in Papadakis $2011^{41}$ to 7354 in Joyce $2008 .^{50}$ All participants were at least 18 years old. The age of the participants in the included studies varied from a mean of 38 to more than 65 years. Six studies included a general population of smokers (Curry 1998; Schauffler 2001; Halpin 2006; Kaper 2006; Patel 2010; Willemsen 2013). ${ }^{42,44,47,48,52,54}$ Dey $1999^{53}$ and Selby $2014^{43}$ included only smokers who were motivated to quit. Half of the sample included in Boyle $2002^{46}$ was motivated to quit smoking. All of the participants in An 2008; Papadakis 2011 and Pakhale 2015 40,41,45 were motivated to quit smoking in the next 30 days. Participants in Hughes $1991^{49}$ did not have to be motivated to quit to participate in the study, but were allowed to withdraw from the study after they were told that they would be randomly assigned to different price groups. Participants were voluntarily enrolled and motivated to quit in Joyce $2008 .^{50}$ Jardin $2014^{39}$ included smokers not wanting to quit in the next 30 days. Three studies assessed the effects of financial interventions directed at healthcare providers (Roski 2003; Twardella 2007; An 2008). 45,51,55 Twardella $2007^{55}$ involved both patient- and healthcare provider-directed interventions. In the studies that involved healthcare provider-directed interventions, patient behavior was measured using a baseline survey and a follow-up survey after six months.

\section{Interventions}

\section{Patient-directed interventions}

Six studies investigated the effect of changes to the level of insurance coverage for smoking cessation treatment (Curry 1998; Schauffler 2001; Boyle 2002; Kaper 2006; Willemsen 2013; Selby 2014). ${ }^{43,44,46,47,52,54}$ Nine studies investigated the effect of changes to the direct cost to the smoker of receiving treatment (Hughes 1991; Dey 1999; Halpin 2006; Twardella 2007; Joyce 2008; Patel 2010; Papadakis 2011; Jardin 2014; Pakhale 2015). ${ }^{39-}$ 42,48-50,53,55 Coverage was offered for four different types of smoking cessation treatment: nicotine replacement therapy (NRT), bupropion, varenicline and behavioral support. Three studies each covered two types of smoking cessation treatment: Boyle $2002^{46}$ offered coverage for NRT (patches and gum) and bupropion, and Curry $1998^{47}$ and Schauffler $2001^{52}$ covered NRT (patches and gum) and participation in a behavioral program. Kaper 2006; Halpin 2006; Twardella 2007 and Joyce 2008 48,50,55,56 covered three types of therapy: NRT (patches, gum, sublingual tablets and lozenges), bupropion and behavioral support. Papadakis $2011^{41}$ and Selby $2014^{43}$ covered NRT, bupropion and varenicline. In Pakhale $2015^{40}$ participants were offered free telephone counselling and they received a USD 110 voucher for pharmacotherapy of their own choice. In Willemsen $2013^{44}$ smokers could be reimbursed by their insurance company for pharmacotherapy if they also engaged in (free) telephone counselling. The treatment periods ranged from two weeks (Jardin 2014) ${ }^{39}$ to one year (Curry 1998; Schauffler 2001; Boyle 2002; Kaper 2006 Joyce 2008 and Selby 2014). ${ }^{43,44,46,47,50,52,54}$ The included studies also varied in the extent of insurance coverage or treatment cost and the comparisons made. Seven studies compared full coverage of the cost of treatment with no coverage (Hughes 1991; Schauffler 2001; Kaper 2006; Twardella 
2007; Joyce 2008; Willemsen 2013; Pakhale 2015). ${ }^{40,44,49,50,52,54,55}$ Five studies reported the effect of partial interventions as compared to no financial intervention (Hughes 1991; Boyle 2002; Papadakis 2011; Jardin 2014; Selby 2014). ${ }^{39,41,43,46,49}$ One study compared full coverage of both behavioral treatment and NRT with a partial benefit requiring a $50 \%$ co-payment for either behavioral or NRT components (Curry 1998) ${ }^{47}$ and two studies investigated the differences between a cost to the patient of USD 20, USD 6/USD 10 or USD 0 per box of nicotine gum (Hughes 1991; Patel 2010). ${ }^{42,49}$ Joyce $2008^{50}$ provided four categories of benefit ranging from usual care to benefits of pharmacotherapy and counselling.

\section{Healthcare provider-directed interventions}

Roski $2003^{51}$ distributed printed versions of smoking cessation guidelines to clinics in both the intervention and control group. The intervention group clinics were eligible for payments for reaching targets for registration of participants' smoking status and providing advice to quit. Twardella $2007^{55}$ provided a tutorial to general practitioners on how to conduct counselling and prescribe pharmacotherapy to help smokers; additionally, the practitioners were provided with a financial remuneration of EUR 130 for each participant they treated and who was biochemically confirmed to have quit at the end of six months' follow-up. An $2008^{45}$ compared usual care and a pay-for-performance intervention offering USD 5000 for 50 quitline referrals. Pay-for-performance clinics also received monthly updates on their referral numbers.

\section{Outcomes}

Abstinence from smoking after six months or more from the start of the intervention was the primary and preferred outcome. Boyle $2002^{46}$ presented self-reported continuous (more than six months) abstinence rates. The longest follow-up report comes from Kaper $2006^{54}$ in which biochemically validated continuous abstinence was reported at 12 months, while Selby $2014^{43}$ reported biochemically validated continuous abstinence for weeks 26-52 and Twardella $2007^{55}$ and Papadakis $2011^{41}$ reported biochemically validated continuous abstinence at six months' follow-up. Seven studies presented self-reported point prevalence abstinence data (Hughes 1991; Curry 1998; Schauffler 2001; Roski 2003; Halpin 2006; Joyce 2008; Pakhale 2015).40,47-52 Three studies reported biochemically validated point prevalence abstinence data (Patel 2010; Papadakis 2011; Selby 2014). ${ }^{41-}$ ${ }^{43}$ In Hughes 1991,49 observers named by the participants were asked to verify reported smoking status of participants. Dey $1999^{53}$ assessed abstinence from smoking at 14 weeks after the start of the reimbursement period and Jardin $2014^{39}$ at 12 weeks; therefore, we did not include data from these studies in the analysis of the effects of reimbursement on abstinence rates. Pakhale $2015^{40}$ had problems with data collection and therefore measured point prevalence abstinence at different endpoints between 26 and 52 weeks, with an average of 33 weeks after baseline. One of the secondary outcome measures was the number of participants who made a quit attempt. Eight studies presented data on this outcome (Hughes 1991; Schauffler 2001; Boyle 2002; Halpin 2006; Kaper 2006; Papadakis 2011; Jardin 2014; Selby 2014). ${ }^{39,41,43,46,48,49,52,54}$ The other secondary outcome measure was the self-reported use or registered use of smoking cessation treatment. This was self-reported in Schauffler 2001; Boyle 2002; Kaper 2006; Papadakis 2011; Jardin 2014 and Pakhale 2015, ${ }^{39-41,46,52,54}$ registered by a health maintenance organization in Curry 
1998, ${ }^{47}$ and by the local pharmacy in Hughes 1991; Dey 1999; Halpin 2006; An 2008 and Selby $2014 .{ }^{43,45,48,49,53}$ Utilization of smoking cessation services was reported by general practitioners in Twardella $2007^{55}$ and by telephone counsellors in Papadakis 2011. ${ }^{41}$ Roski $2003^{51}$ recorded self-reports by smokers regarding the use of bupropion or NRT and/or any counselling services. Willemsen $2013^{44}$ used data on treatment enrolment recorded by the national quitline. Patel $2010^{42}$ only registered the number of nicotine boxes acquired by participants and not actual medication use. Eight of the 17 included studies presented data on the costs of the intervention, and compared the costs and effects of the intervention with one or two alternatives (Hughes 1991; Curry 1998; Schauffler 2001; Halpin 2006; Kaper 2006; Twardella 2007; An 2008; Joyce 2008). ${ }^{45,47-50,52,54,55}$ Most of the studies used a time horizon equal to the duration of the intervention, and all used a third-party payer perspective, in which only the direct costs of the intervention were presented. Curry $1998^{47}$ also presented a user's perspective. The cost effectiveness ratio was presented in terms of costs per user who quit smoking or costs per participant enrolled. No study presented data in terms of quality-adjusted life years (QALY) saved. For Kaper $2006,{ }^{56}$ additional information on outcomes on quit attempts, use of treatment and cost were collected from two other related reports (Kaper 2005a, Kaper 2006a). ${ }^{56,57}$

\section{Excluded studies}

Of the 60 studies for which we assessed full reports for eligibility, we excluded 45 . Six studies were research protocols without data (Courtney 2014; Ostroff 2014; Harter 2015; Bonevski 2016; Park 2016; NCT00962988)..$^{58-63}$ Although NCT00962988 ${ }^{63}$ and Ostroff $2014^{61}$ had not yet been published at the time of writing this review, we assessed them as probably relevant and therefore registered as ongoing studies. Most excluded studies were not randomised controlled trials, controlled trials or interrupted time series (ITS) studies (Alberg 2004; Cummings 2006). ${ }^{34,64}$ Oswald $1988^{65}$ and Cox $1990^{13}$ retrospectively compared the outcomes of using free and purchased gum in a non-randomized trial. Russos 1999; Parnes 2002 and Stone $2002^{66-68}$ all used a cross-sectional design. Bailey $2016^{69}$ was a cohort study and Hamilton $2013^{70}$ a systematic review. Fiore 2000, Coleman 2001, Doescher 2002, Latts 2002, Ringen 2002, Solberg 2002, Amundson 2003, Chang 2008 and Weisman $2012^{11,71-78}$ did not have a control group and did not use an ITS design. Land $2010^{79}$ used an ITS design but did not meet the required inclusion criterion of three measuring points before and three after the intervention for the outcomes quit attempts and quit success and we therefore excluded it. There were other reasons for exclusion in addition to study design. Lave $1996^{80}$ compared two different financial systems in two different settings, and did not report data on the smoking status of the control group. The financial intervention in nine studies (Curry 1991; Hovell 1996; Russos 1999; Donatelle 2000; Pardell 2003; Volpp 2006; Kruse 2013; McLeod 2015; Moskowitz 2016) ${ }^{67,81-88}$ was not directly related to the use of smoking cessation treatment. Krist $2010^{89}$ was excluded since its intervention of free counselling was not directed specifically at smoking but also at 'unhealthy' behaviors such as drinking alcohol. Hays $1999^{90}$ and Hockenberry $2012^{91}$ were excluded because they did not explicitly and directly assess the effects of a financial intervention. Shaw $2003^{92}$ assessed the effect of nicotine gum prices on the use of gum and abstinence rates, and did not report the number of participants using smoking cessation treatment or the quit rate. We excluded Walsh 2012; Amemori 2013; Bardach 2013 and Verbiest 2013 ${ }^{93-96}$ because they did not measure relevant outcome measures 
for this review. Fu $2016^{97}$ was aimed at assessing the effect of proactive counselling and lacked a suitable control group.

\section{Risk of bias in included studies}

The summary results of our methodological assessment are displayed in Figure 1.

\section{Allocation}

Ten studies stated that individual participants were randomly allocated to the different benefit groups (Hughes 1991; Dey 1999; Schauffler 2001; Halpin 2006; Kaper 2006; Patel 2010; Papadakis 2011; Jardin 2014; Selby 2014; Pakhale 2015). ${ }^{39-43,48,49,52,53,56}$ Three studies randomly allocated clinics to study conditions and then identified smokers (Roski 2003; Twardella 2007; An 2008). ${ }^{45,51,55}$ One study conducted randomization based on the location or geography of the primary care clinics (Joyce 2008)..$^{50}$ Although the exact method for generating the randomisation sequence was generally not reported, we classified all these 14 studies as having a low risk of bias for this part of the design. Three studies (Curry 1998; Boyle 2002 and Willemsen 2013) ${ }^{44,46,47}$ were not randomized trials and did not involve experimental manipulation of conditions. Willemsen $2013^{44}$ used a natural on-off design in the entire Dutch population and we classified it as having an unclear risk of bias. Boyle $2002^{46}$ and Curry $1998^{47}$ made use of natural experiments in which different insured groups were receiving different smoking cessation benefits. This had the potential for bias due to differences between people in different benefit groups, and therefore we classified these two studies as a having a high risk of bias. Five studies reported allocation concealment with enough detail to judge there to be a low risk of selection bias (Hughes 1991; Dey 1999; Papadakis 2011; Selby 2014; Pakhale 2015). ${ }^{40,41,43,49,53}$ Six studies did not give enough information on allocation concealment procedure and we therefore assessed them as having an unclear risk of bias (Schauffler 2001; Halpin 2006; Jardin 2014; Patel 2010; An 2008). ${ }^{39,42,45,48,52}$ We judged five studies to be at high risk of bias (Curry 1998; Boyle 2002; Kaper 2006; Twardella 2007; Joyce 2008). ${ }^{46,47,50,55,56}$ For Curry $1998^{47}$ and Boyle $2002^{46}$ allocation concealment was not directly applicable because benefit groups were not experimentally manipulated, for the other three studies, we judged allocation concealment to be inadequate. For Willemsen $2013^{44}$ allocation concealment was not applicable because of its ITS design.

\section{Blinding}

Kaper $2006^{56}$ blinded the participants in the control group to the treatment available to the experimental group and evaluated the success of the blinding and concluded that it was successful. Therefore, this was the only study that we assessed as being at low risk of bias. We judged three studies to be at unclear risk of bias due to lack of information provided. We judged 13 studies to be at high risk of bias because of incomplete) or no blinding (An 2008; Boyle 2002; Curry 1998; Dey 1999; Halpin 2006; Hughes 1991; Joyce 2008; Pakhale 2015; Papadakis 2011; Roski 2003; Schauffler 2001; Selby 2014; Twardella 2007). ${ }^{40,41,43,45-53,55}$ In Papadakis $2011^{41}$ clinicians and participants were not blinded, but the research coordinator conducting the outcome assessment was blind to group allocation. Selby $2014^{43}$ unblinded the investigator and participant before deciding on the smoking cessation method to be used, but both were blinded to the results of cotinine tests. 


\section{Incomplete outcome data}

We assessed eight studies to be at low risk of attrition bias (Hughes 1991; Curry 1998; Halpin 2006; Kaper 2006; An 2008; Papadakis 2011; Jardin 2014; Pakhale 2015);39-41,45,47-49,56 the other studies we assessed to be at high risk of attrition bias. In Boyle $2002^{46}$ participants were self-selected respondents to a survey so an ITT analysis was not possible. In addition, the study authors did not account for $20 \%$ 'unusable sample' excluded from the analysis. Dey $1999^{53}$ and Joyce $2008^{50}$ had different attrition rates in the experimental and control groups, and did not provide an explanation for this difference, so it was unclear whether attrition was at random or not. Patel $2010^{42}$ reported significant different attrition rates between experimental groups. In Roski $2003^{51}$ it was not reported how non-response or loss to follow-up were handled in the analysis. Schauffler $2001^{52}$ did not provide sufficient information on missing data and did not report an ITT. In Selby $2014^{43}$ a higher proportion of the participants in the control group discontinued the study because they were 'no longer willing to participate.' The follow-up rate was less than $80 \%$ for all the included studies except in Curry 1998; Halpin 2006; Twardella 2007 and Jardin 2014, ${ }^{39,47,48,55}$ which had higher rates. In Patel $2010^{42} 55 \%$ of the participants in the control group compared to $13 \%$ in the intervention group withdrew from the study. Five studies did not address incomplete data (Dey 1999; Schauffler 2001; Boyle 2002; Roski 2003; Twardella 2007). ${ }_{4}^{46,51-}$ 53,55 Hughes 1991; Halpin 2006; Kaper 2006; Papadakis 2011; Jardin 2014 and Selby $2014^{39,41,43,48,49,56}$ used an ITT analysis.

\section{Other potential sources of bias}

We classified six studies as being at high risk of another source of bias (Dey 1999; Boyle 2002; Roski 2003; Kaper 2006; Willemsen 2013; Pakhale 2015). ${ }^{40,44,46,51,53,56}$ In Boyle 2002; Kaper 2006 and Pakhale $2015^{40,46,56}$ self reports of quitting or abstinence were not properly validated. Dey $1999^{53}$ lacked power and reported only short-term abstinence. Roski $2003^{51}$ reported a possible Hawthorne effect on all clinics regardless of randomization status. A potential source of bias for the ITS study of Willemsen $2013^{44}$ is that the start of the reimbursement period was accompanied by a large media campaign to encourage smokers to apply for the free smoking cessation program. We judged eight studies to be at unclear risk of bias (Hughes 1991; Curry 1998; Schauffler 2001; Halpin 2006; An 2008; Joyce 2008; Patel 2010; Papadakis 2011).41,42,45,47-50,52 There was no or unclear baseline comparability reported with regard to important predictors such as smoking levels and dependence, age, sex, income etc. in four studies (Hughes 1991; Boyle 2002; Roski 2003; Joyce 2008). ${ }^{46,49-51}$ In Papadakis $2011^{41}$ participants in the intervention group reported smoking more cigarettes per day and they also reported a higher self-efficacy with quitting than participants in the control group. Patel $2010^{42}$ reported a higher mean number of cigarettes smoked per day in the intervention group and an unequal distribution of male/ female participants across intervention groups.

\section{Quality of economic evaluation studies}

The methodological quality assessment regarding the economic evaluations is presented in Appendix 3. The score of the five studies varied between 6 and 15 (out of a possible maximum of 19). In only one study were all relevant costs identified, measured and valued appropriately (Kaper 2006). ${ }^{56}$ For example, costs of visits to healthcare providers were not measured, no contact times were presented, the volumes of the use of smoking cessation 
treatment were incomplete and the sources of cost valuation were not described. Incremental analyses and sensitivity analyses were not performed. Direct costs were not discounted, but this was appropriate as the time frame of the cost analysis was less than 12 months. No statements of potential conflicts of interest were presented.

\section{Effects of interventions}

To determine the general effect of healthcare financing interventions, we performed meta-analysis using a random-effects model. When only one study examined the effects of an intervention on a specific outcome, we presented the results of this individual study graphically.

\section{The effect of financial interventions directed at smokers}

\section{Abstinence from smoking}

In this section we report the effect of financial interventions directed at smokers on continuous and point prevalence abstinence rates. The reported abstinence rates were stratified into four different intervention subgroups and the pooled effect of each of the financial benefit combinations is reported.

\section{Full coverage versus no financial intervention}

Six studies reported the effect of full versus no financial intervention. Among these, two reported biochemically validated continuous abstinence rates at six months (Twardella 2007) $)^{55}$ and one year (Kaper 2006)..$^{56}$ The other studies reported six-month (Hughes 1991; Schauffler 2001) ${ }^{49,52}$ and 12-month (Joyce 2008) ${ }^{50}$ self-reported point prevalence abstinence, and abstinence between 26 and 52 weeks (Pakhale 2015), ${ }^{40}$ which we considered as sixmonth abstinence. In all six studies the abstinence rate favored the intervention group, however, only Schauffler 2001; Kaper 2006 and Joyce 2008 50,52,56 reached statistical significance. Upon pooling of the six studies there was a statistically significant favorable effect of financial interventions on abstinence but with a moderate level of heterogeneity (RR $1.77,95 \% \mathrm{Cl} 1.37$ to $2.28, \mathrm{IO}=33 \%, 9333$ participants) (Analysis 1.1 ).

\section{Full coverage versus partial coverage interventions}

Five studies reported the effect of full compared to partial financial interventions. One study reported six-month CO-validated continuous abstinence (Papadakis 2011), ${ }^{41}$ the other studies reported six-month (Curry 1998; Hughes 1991), ${ }^{47,49}$ eight-month (Halpin 2006) ${ }^{48}$ and 12-month (Joyce 2008) ${ }^{50}$ self-reported point prevalence abstinence. Two of the studies, Hughes 1991 and Joyce 2008,49,50 had point estimates favoring the full intervention group but only Joyce $2008^{50}$ reached statistical significance. Papadakis $2011^{41}$ was underpowered to find statistically significant results. The overall pooled estimate did not show an additional positive effect of full interventions on top of partial interventions on smoking abstinence (RR $1.02,95 \% \mathrm{Cl} 0.71$ to $1.48,12=64 \%, 5914$ participants) (Analysis 1.2).

\section{Partial coverage versus another partial coverage intervention or no intervention}

Five studies reported the effect of partial financial intervention as compared to no financial intervention on abstinence from smoking. Selby $2014^{43}$ reported 12-month continuous abstinence and Boyle $2002^{46}$ and Joyce $2008^{50}$ reported 12-month point 
prevalence abstinence. Two studies reported six-month point prevalence abstinence: Hughes $1991^{49}$ and Patel $2010^{42}$ (CO-validated). The pooled estimate showed a favorable effect of financial intervention on abstinence rates (RR $1.27,95 \% \mathrm{Cl} 1.02$ to $1.59, \mathrm{I}=21 \%$, 7108 participants) (Analysis 1.3). When assessing the effect of financial interventions involving one type of partial financial intervention compared with another, where the partial intervention group with more benefits was considered an experimental group, Curry $1998^{47}$ was the only study that provided self-reported, six-month point prevalence abstinence. There was no statistically significant difference between the two intervention strategies on abstinence rates (RR 1.20, 95\% Cl 0.86 to 1.68, 298 participants) (Analysis 1.4).

\section{Number of participants making a quit attempt}

Four studies evaluated the impact of full financial interventions versus no intervention on quit attempts (Hughes 1991; Schauffler 2001; Kaper 2006; Joyce 2008). ${ }^{49,50,52,98}$ All four studies indicated a favorable effect of full financial intervention; this effect was statistically significant in Schauffler $2001^{52}$ and Joyce 2008. ${ }^{50}$ When the estimates were pooled there was a small positive effect of full benefit on the rate of quit attempts with RR of 1.11 (95\% Cl 1.04 to $1.17,12=15 \%, 9065$ participants) (Analysis 2.1). Four studies assessed the impact of full versus partial financial interventions on the rate of quit attempts (Hughes 1991; Halpin 2006; Joyce 2008; Papadakis 2011). ${ }^{41,48-50}$ Joyce $2008^{50}$ showed a small favorable effect of full financial incentives on quit attempts, but the pooled estimate did not show a statistically significant effect (RR $0.99,95 \% \mathrm{Cl} 0.84$ to $1.17,12=57 \%, 5486$ participants) (Analysis2.2). Five studies reported the effect of partial financial interventions as compared to no financial benefit on the rate of quit attempts (Hughes 1991; Boyle 2002; Joyce 2008; Jardin 2014; Selby 2014)..$^{39,43,46,49,50}$ All studies had confidence intervals including the line of no effect except for Selby 2014, which showed a statistically significant beneficial effect of financial intervention. The pooled estimate marginally favored partial financial interventions but was not statistically significant (RR $1.13,95 \% \mathrm{Cl} 0.98$ to $1.31,12=88 \%$, 6944 participants) (Analysis 2.3). However, the studies showed substantial statistical heterogeneity.

\section{Use of smoking cessation treatment}

In this section we present the pooled estimates of financial interventions on the use of the smoking cessation treatments such as nicotine replacement therapy (NRT), bupropion, varenicline and behavioral interventions.

\section{Full coverage versus no financial intervention}

Seven studies reported outcomes on the number of smokers using smoking cessation treatment by study groups with full financial benefit compared to those with no benefit. The overall or pooled effect of full financial benefit compared to no benefit on the use of NRT, bupropion and behavioral interventions was positive and significant in each subgroup, though we could not pool the individual subgroups as four studies were included in multiple subgroups (Analysis 3.1). The studies by Hughes 1991; Dey 1999; Schauffler 2001; Kaper 2006; Twardella 2007; Joyce 2008 and Pakhale 201540,49,50,52,53,55 reported the utilization rate of NRT. In all studies full financial interventions increased the use of NRT except for Pakhale $2015,{ }^{40}$ which showed a positive effect on NRT utilization but did not reach statistical significance. The pooled estimate indicated a significantly higher 
use of NRT in participants receiving full interventions with a RR of 1.79 (95\% Cl 1.54 to 2.09, $12=34 \%, 9455$ participants) (Analysis 3.1). Three studies reported the rate of utilization of bupropion (Kaper 2006; Twardella 2007; Joyce 2008). ${ }^{50,55,56}$ All the studies showed an increased use of bupropion in the financial intervention group. The pooled estimate also showed a large and significant positive effect of full financial intervention on the use of bupropion treatment with a RR of 3.22 ( $95 \% \mathrm{Cl} 1.41$ to $7.34,12=71 \%, 6321$ participants) (Analysis 3.1). However, the high heterogeneity calls for a cautious interpretation. Four studies reported on the utilization of behavioral interventions (Schauffler 2001; Kaper 2006; Twardella 2007; Joyce 2008). ${ }^{50,52,55,56}$ Kaper $2006^{56}$ and Joyce $2008^{50}$ indicated a positive effect of full financial intervention on the use of behavioral smoking cessation therapy; Schauffler $2001^{52}$ and Twardella $2007^{55}$ also showed a positive effect on the use of therapy but the results were statistically non-significant. Upon pooling, there was substantial heterogeneity, but financial interventions had a statistically significant positive effect of on the use of behavioral therapy (RR 1.77, 95\% Cl 1.19 to $2.65,12=75 \%$, 9215 participants) (Analysis 3.1). One study reported the combined use of NRT and oral medications (Pakhale 2015) ${ }_{1}^{40}$ which did not differ significantly between research groups that received a financial intervention and that did not (RR $1.11,95 \% \mathrm{Cl} 0.73$ to 1.68).

\section{Full coverage versus partial coverage interventions}

Six studies reported the impact of full compared to partial financial interventions on the utilization of pharmacotherapy and behavioral support (Hughes 1991; Curry 1998; Halpin 2006; Joyce 2008. Papadakis 2011; Willemsen 2013).41,44,47-50 Because of its ITS design, we did not include Willemsen $2013^{44}$ in the subgroup analysis. As Curry $1998^{47}$ was included in multiple subgroups in this analysis, we were unable to pool the results and report usage of each type of therapy separately. One study showed a large and statistically significant positive effect of full interventions on the use of NRT (Curry 1998).$^{47}$ Joyce $2008^{50}$ was also significant, but the remaining two studies had similar sized smaller effects that were not statistically significant. There was a high level of heterogeneity in the estimate of the pooled effect of the four studies in regard to effect on use of NRT (RR 1.76, 95\% Cl 1.27 to $2.43,12$ $=87 \%, 22,380$ participants) (Analysis 3.2). Pertaining to use of behavioral interventions, there was a significantly increased use in Curry $1998^{47}$ (RR 3.95, 95\% Cl 3.15 to 4.95, 16,922 participants) - the only study in this category. Willemsen $2013^{44}$ measured the number of people using behavioral therapy via the national quitline before and after the introduction of a national reimbursement system. The number of participants increased from 848 smokers enrolled in the year before the reimbursement to 9091 smokers enrolled during the year after reimbursement was instated. When the reimbursement was discontinued, only 151 smokers enrolled in the first 18 weeks of that year. Both Halpin $2006^{48}$ and Joyce $2008^{50}$ reported the utilization of bupropion, in which there was no statistically significant effect of full financial intervention as compared with partial financial intervention with a RR of 1.42 ( $95 \%$ Cl 0.84 to 2.41, I2 = 61\%, 3700 participants) (Analysis 3.2) upon pooling. Papadakis $2011^{41}$ had a very small sample size and showed no significant results on the use of pharmacotherapy in general (RR $1.19,95 \% \mathrm{Cl} 0.70$ to $2.02,28$ participants). 
Partial coverage versus another partial coverage intervention or no financial intervention Hughes 1991; Boyle 2002; Joyce 2008; Jardin 2014 and Selby 201439,43,46,49,50 reported on the effect of partial as compared to no financial intervention on utilization of pharmacotherapy. The pooled estimate of the five studies showed a small positive but not statistically significant effect of the intervention on utilization of NRT with a RR of 1.37 (95\% Cl 0.99 to $1.91,12=91 \%, 6944$ participants) (Analysis 3.3). Unlike the other studies where participants had to take action themselves to receive pharmacotherapy, in Jardin $2014^{39}$ participants in the intervention group were sent a two-week supply of NRT by mail, which could make the results not entirely comparable with the other studies. If Jardin $2014^{39}$ is left out of the pooled results, the RR declines to 1.13 (95\% Cl 0.88 to 1.45 , $12=86 \%, 6840$ participants) (Analysis 3.3). Boyle 2002; Joyce 2008 and Selby 201443,46,50 presented the effect of partial interventions on bupropion use. Members of the group with coverage for pharmacotherapy had a slightly higher use of bupropion than those without (RR $1.15,95 \% \mathrm{Cl} 1.03$ to $1.29,12=0 \%, 6765$ participants) (Analysis 3.3). A single study reported the effect of a partial financial intervention on the use of varenicline (Selby 2014). ${ }^{43}$ The analysis showed a positive result on the use of varenicline (RR $1.85, \mathrm{Cl} 1.68$ to $2.03,1380$ participants) (Analysis 3.3). Only the small study of Jardin $2014^{39}$ reported the use of behavioral interventions, which was not affected by the financial intervention (RR $0.77,95 \% \mathrm{Cl} 0.22$ to $2.71,104$ participants) (Analysis 3.3). Curry $1998^{47}$ was the only study that reported the impact of partial versus other partial financial interventions on the use of pharmacotherapy and behavioral support. It did not show an effect on the use of NRT or behavioral interventions (RR $0.83,95 \% \mathrm{Cl} 0.68$ to 1.02 and $\mathrm{RR} 0.82,95 \% \mathrm{Cl} 0.61$ to 1.11 , 14,155 participants) (Analysis 3.4).

\section{The effect of financial interventions directed at healthcare providers}

Three studies reported the effect of financial interventions directed at healthcare providers (Roski 2003; Twardella 2007; An 2008)..$^{45,51,55}$ Roski $2003^{51}$ and Twardella $2007^{55}$ reported outcomes on abstinence. Neither showed statistically significant effects. When the effects of financial interventions on abstinence were pooled the results showed that interventions targeting healthcare providers did not affect abstinence from smoking (RR $1.16,95 \% \mathrm{Cl} 0.98$ to $1.37,12=0 \%, 2311$ participants) (Analysis 4.1 ). In the same two studies, financial interventions did not influence the use of pharmacotherapy (NRT) when pooled, with a RR of 0.94 (95\% Cl 0.76 to $1.18,12=6 \%, 2311$ participants) (Analysis 4.2). However, there was a statistically significant positive effect of interventions directed at healthcare providers on the use of behavioral support when the three studies (Roski 2003; Twardella 2007; and An 2008) ${ }^{45,51,55}$ were combined (RR 1.69, 95\% Cl 1.01 to $1.28,12=85 \%, 25,820$ participants) (Analysis 4.3).

\section{The cost effectiveness of financial interventions}

Data on the costs of the intervention were available for eight studies (Hughes 1991; Curry 1998; Schauffler 2001; Halpin 2006; Kaper 2006; Twardella 2007; An 2008; Joyce 2008).45,47$50,52,55,56$ All studies had interventions directed at smokers and Twardella $2007^{55}$ also included an intervention directed at physicians. As pooling of the different economic evaluations is only allowed when there is no interaction between the setting and the effect of the intervention (Drummond 2015), ${ }^{35}$ we did not pool the results of the individual studies. For the most part, the review authors presented smoking-related outcomes and performed 
the incremental analyses. One study calculated the costs per QALY saved (Kaper 2006). ${ }^{56}$ Hughes $1991^{48,49}$ included the following direct costs: nicotine gum, a smoking cessation booklet and healthcare providers' time. Participants' time was regarded as an indirect cost. The calculated financial gain per participant enrolled was USD 1120 with full coverage when gum was provided free, USD 280 when gum was provided at a cost of USD 6 per box and USD 413 when gum cost USD 20. For the incremental analyses, we calculated the costs per additional quitter for the different comparisons. When we compared a full incentive with a partial incentive, the costs per additional quitter were USD 260. When we compared a full incentive with no incentive, the costs were USD 716. A partial incentive was not cost effective when compared with no incentive. Curry $1998^{47}$ presented the direct costs of NRT and a behavioral intervention program for the different coverage groups. Indirect costs were not registered. With full coverage, the average costs per benefit user who quit were USD 21 for users and USD 1117 for the health plan. With partial coverage, the costs per benefit user who quit were respectively USD 326 and USD 801. We also calculated the incremental cost-effectiveness ratio: if full coverage were introduced instead of partial coverage, the financial gain for users would be USD 5316 for each benefit user who quit. For the health plan, the costs would be USD 7646 per benefit user who quit. Schauffler $2001^{52}$ reported the total costs of NRT, the behavioral program and the self-help kit for the treatment group, but not for the control group. The study authors have subsequently advised us that the control group costs amounted to USD 29 per participant, for the selfhelp kit. The average costs per quitter were USD 1495. The costs per additional quitter for full coverage compared with no coverage were USD 1247. Halpin $2006^{48}$ calculated the cost of treatment (self-help kit, NRT and proactive telephone counselling). The cost per prevalent abstinence at eight months were USD 449 per quitter in the 'drugs only' group and USD 842 in the 'drugs and counselling' group. The incremental cost-effectiveness ratio we calculated was USD 731 for each additional quitter in the'drugs and counselling' group. Kaper 2006 reported the costs of treatment per participant as USD 378 and USD 491 in the control group and intervention group, respectively. The costs per additional quitter were calculated by bootstrap replicated with a mean cost-effectiveness ratio of USD 1453 per quitter. The mean costs per additional QALY were USD 2342. If society is willing to pay USD 12,990 for an additional 12-month quitter, the probability that reimbursement for smoking cessation treatment would be cost effective was $95 \%$. Twardella $2007^{55}$ (as reported in Salize 2009) indicated that the costs of treating a participant ranged from USD 0 in the treatment-as-usual group to USD 2039 in the training and incentive group, USD 6404 in the training and medication group and USD 12,821 in the training, incentive and medication group. We used the bootstrap replication method to calculate the costs per additional quitter, which were USD 108 and USD 97 in the two interventions compared to treatment as usual. An $2008^{45}$ indicated that the marginal cost for the intervention clinics was USD 83 per additional referral and USD 300 per additional enrollee for treatment. In Joyce $2008{ }^{50}$ the additional cost per quitter in the intervention groups relative to the usual-care group ranged from USD 463 to USD 6450 per quitter. The costs escalated with increased use of resources for treatment. 


\section{DISCUSSION}

We identified 14 RCTs, two controlled natural experiments and an ITS study to answer questions on the importance of financial interventions directed at smokers and healthcare providers in increasing abstinence from smoking, quit attempts by smokers, and the use of smoking cessation treatment, and where possible to assess their cost effectiveness. We investigated the effect of financial interventions by identifying comparison subcategories between full, partial, and no financial intervention. Full financial benefits directed at smokers provided very positive outcomes compared to no benefits on continuous abstinence, point prevalence abstinence, and utilization of smoking cessation treatment. When compared to no benefits, full financial coverage showed a modest but positive effect on quit attempts. Though not consistently, full financial benefits seem to also have more beneficial effect than partial coverage. We detected a mixed effect of the different levels of financial interventions with regard to the different endpoints with low to high levels of between-studies variance (heterogeneity). There is scant evidence to pass judgement on financial interventions directed at healthcare providers, but the available evidence shows only limited impact on smoking cessation.

\section{Summary of main results}

We detected a statistically significant, positive effect of full financial interventions targeting smokers with regard to abstinence from smoking compared to provision of no financial intervention at six months' follow-up or more (all abstinence measures: RR 1.77, $95 \% \mathrm{Cl} 1.37$ to $2.28, \mathrm{IO}=33 \%, 9333$ participants). The effect of full financial interventions was also extended to favorable outcomes on the use of smoking cessation treatments: the pooled effect of full coverage compared with no financial intervention on the use of smoking cessation treatments was highly significant for each treatment type (NRT, bupropion, and behavioral interventions). Full financial interventions had a small, significant effect on quit attempts (RR 1.11, Cl 1.04 to $1.17, \mathrm{IO}=15 \%, 9065$ participants). When full coverage was compared to partial coverage, results showed no significant additional effect of full coverage on smoking cessation or quit attempts. Pooling together results from four trials, full coverage did increase the use of NRT when compared with partial coverage, though the level of heterogeneity was high $(1 \mathrm{O}=87 \%)$. There were not enough data to investigate the effects of full coverage versus partial coverage on the utilization of bupropion or behavioral therapy. These findings could mean that full financial interventions may have a significant effect over partial interventions regarding the use of treatment. However, these findings should be interpreted with caution given the high heterogeneity, which reflects the reality that partial interventions could come in various shapes and combinations. Upon pooling, we found a small positive effect of partial financial interventions compared to no interventions on abstinence (RR 1.27, Cl 1.02 to $1.59, \mathrm{IO}=21 \%, 7108$ participants). We did not detect an effect of partial financial interventions compared to other partial or no financial interventions on attempts at quitting. There may be an effect of partial financial interventions when compared to no interventions with regard to the use of pharmacotherapy. Upon the pooling of three studies, the use of bupropion was increased (RR 1.15, 95\% Cl 1.03 to $1.29,10=0 \%, 6765$ participants), The use of NRT was also increased but pooling of the studies just failed to reach statistical significance (RR $1.37,95 \% \mathrm{Cl} 0.99$ to $1.91, \mathrm{IO}=91 \%, 6944$ participants). The 
single study that investigated varenicline (Selby 2014) showed a positive effect on its use (RR $1.85,95 \% \mathrm{Cl} 1.68$ to $2.03,1380$ participants). Only three included studies were directed at healthcare providers, which should be considered when interpreting the results. We detected no effect of healthcare provider-directed benefits on continuous abstinence, point prevalence abstinence or on the use of NRT. However, healthcare provider-directed interventions compared to no intervention increased the use of behavioral therapy (RR $1.69,95 \% \mathrm{Cl} 1.01$ to $2.86, \mathrm{IO}=85 \%, 25,820$ participants). Financial benefits may induce healthcare providers to provide behavioral-intervention support to smokers, though the high level of heterogeneity suggests results should be interpreted carefully. It should also be borne in mind that financial resource should not be invested merely for increasing smoking cessation treatments unless it can be translated into favorable quitting rates. Generally speaking, our findings seem to support the general trend of mixed and nonconclusive findings on healthcare provider-directed financial interventions for improving patient outcomes (Steel 2007; Peckham 2010; Van Herck 2010; De Bruin 2011; Flodgren 2011; Ryan 2016). ${ }^{18,19,21-24}$ Eight studies (Hughes 1991; Curry 1998; Schauffler 2001; Halpin 2006; Kaper 2006; Twardella 2007; An 2008; Joyce 2008) ${ }^{45,47-50,52,55,56}$ presented data on cost effectiveness. When full benefit was compared with partial or no benefit, the costs per quitter ranged from USD 97 to USD 7646. We conclude that full financial interventions directed at smokers when compared to no financial interventions can increase the rate of successful quitting, quit attempts and utilization of pharmacotherapy in smokers. Although the absolute differences were small, the costs per additional quitter were low. We did not detect a difference in effect between full and partial financial interventions in abstinence from smoking or increased quit attempts, but we did find a significant difference in effect between full and partial interventions in use of NRT and behavioral therapy. Partial versus no financial coverage showed an increase in abstinence and quit attempts and in the use of bupropion and varenicline. There is inadequate evidence to determine the effect of partial financial interventions when compared to other partial financial interventions or no financial interventions on the other outcomes. The conclusions need to be interpreted in light of the reservations discussed below with regard to comparability, classification and methodological quality.

\section{Overall completeness and applicability of evidence}

Despite the creation of relatively homogeneous subcategories for analyses based on theoretical and practical considerations, the studies are still heterogeneous with respect to the study setting, motivation to participate in the study and motivation to quit smoking. Because of such sources of heterogeneity, the results of the meta-analysis have to be interpreted with care. The setting of the included studies ranged from family practices and hospital clinics in the UK, USA and Canada, to health insurance organizations in the USA, Germany and the Netherlands. As each country has a different healthcare system, comparisons between studies in various settings should be made in the knowledge of these differences. In Dey 1999,53 for example, participants had to be motivated to quit in order to participate. On entry into the study, motivated participants received free prescriptions for nicotine patches, and as a result, $97 \%$ of the participants in the full incentive group used at least one prescription. Such differences potentially limit the interpretability of the effect size estimates that come out of pooling. On the other hand, Kaper $2006^{56}$ and Schauffler $2001^{52}$ offered coverage in a general population. As participants were not enrolled based 
on motivation to quit smoking, the use of NRT among the intervention groups was $20.6 \%$ in Schauffler $2001^{52}$ and $4 \%$ in Kaper $2006 .{ }^{56}$ Furthermore, the interventions varied in the extent of financial benefit, the methods of smoking cessation treatment for which the benefit was available, the conditions for receiving the benefit and the information concerning the new benefit. In 12 of the 14 patient-directed financial intervention studies, a financial benefit was available for different types of smoking cessation treatment. In two studies (Halpin 2006 and Twardella 2007) ${ }^{48,55}$ the trial arms did not enable us to examine the independent effect of financial interventions and hence were excluded from the analysis. In Twardella 2007,,5 one of the arms involved both patient-directed and healthcaredirected interventions, while in Halpin $2006,{ }^{48}$ special constraints were put on participants where they were required to attend counselling before free medications were financed. During subgroup analyses, the main emphasis was on the level of access to smoking cessation treatments: full, partial or no access through financing to enable smokers to quit. Partial interventions could refer to either pharmacotherapy or counselling only. In our subgroup analyses no further stratification was considered for partial interventions for smoking cessation since, in all studies, partial interventions included pharmacotherapy only except Joyce $2008 . .^{50}$ As a result, there is limited confounding by type of treatment provided in the partial intervention group. However, future systematic reviews, with the hope that additional studies would accumulate, need to further stratify partial treatment benefits highlighting the type of smoking cessation treatments covered, at least based on behavioral interventions and pharmacotherapy. This is due to the fact that there could be differences in effect between counselling and pharmacotherapy. It is fair to expect that the kind of pharmacotherapy covered could also influence the outcomes of the financing interventions. All patient-directed studies included in this review covered NRT while nine studies (Boyle 2002; Halpin 2006; Kaper 2006; Twardella 2007; Joyce 2008; Papadakis 2011; Willemsen 2013; Selby 2014; Pakhale 2015) ${ }^{40,41,43,44,46,48,50,55,56}$ also covered bupropion therapy and four studies additionally covered varenicline (Papadakis 2011; Willemsen 2013; Selby 2014; Pakhale 2015). ${ }^{40,41,43,44}$ An example of different conditions for receiving a financial benefit is related to voluntary or obligatory visits to healthcare providers. In Kaper 2006, ${ }^{56}$ participants received coverage after a statement of contact with a healthcare provider was sent to the health insurance company. The use of behavioral support in Kaper $2006^{56}$ is therefore not comparable with Schauffler $2001^{52}$ or Joyce $2008,{ }^{50}$ in which some or all of the participants voluntarily chose to participate in a behavioral support intervention. There were also differences in the information provided to participants about their benefit and the extension of the benefit. In Boyle 2002, ${ }^{46}$ for example, participants were not explicitly informed of the intervention, and as a result only $30 \%$ of smokers in the treatment group knew about the offer of financial benefit. This may be the reason for the statistically non-significant effects found in Boyle $2002^{46}$ when compared with other studies, in which participants were informed about their new benefit. Participants' awareness of the available benefits could contribute significantly to an increase in the effect of the intervention, as it would likely increase the use of smoking cessation treatments and hence increase the absolute number of quitters. The term 'full intervention' should be interpreted with caution as it is not synonymous with optimal level of care. Furthermore, an intervention classified as partial does not exclude the possibility that some motivated participants may have used additional treatment to complement those financed (e.g. Schauffler 2001)..$^{52}$ This phenomenon has also been documented 
by other investigators (Hall 2002). ${ }^{99}$ As utilization of smoking cessation treatment was loosely investigated using patient and physician reports or care provider registries, the actual utilization rates of the services may be lower or higher. It is also difficult to estimate adherence to a prescribed pharmacotherapy from the included studies. As a result, such complexities could have influenced the efficacy of the interventions.

\section{Quality of the evidence}

An important limitation of the included studies was that not all used random or concealed allocation. Twelve studies randomly allocated the participants to the treatment or control groups, and only five of these employed concealed allocation. Particularly in Boyle $2002^{46}$ and Curry $1998,{ }^{47}$ there is the possibility of selection bias as the studies were natural experiments. In the remaining studies, the possibility exists that the effect is biased. Furthermore, not all of the studies blinded the participants, healthcare providers or outcome assessors. In studies that assess the effect of a financial intervention, blinding the control group may be important, since control participants who knew that they would not receive a financial benefit for treatment might have felt disadvantaged and changed their behavior. Such a change would be a threat to the validity of the study. We therefore could not rule out the possibility of biased results in the unblinded studies. However, we also acknowledge that financial interventions may be more difficult to blind than drug interventions. Another methodological issue in the included studies is the low follow-up rate, which was below $80 \%$ in most of the included studies. This may be related to the type of intervention. If, for example, participants are less interested in the financial intervention when they do not want to use smoking cessation treatments or are not motivated to quit, then the number of dropouts could increase. In a conservative analysis, dropouts would be considered to be continuing smokers. However, such analyses were not performed in five studies (Dey 1999; Schauffler 2001; Boyle 2002; Roski 2003; Twardella 2007). ${ }^{46,51-53,55}$ For this review, we have rectified the problem by recalculating the results of the studies using an ITT analysis that counted dropouts and participants lost to follow-up as continuing smokers who had not attempted to quit and not used the treatment(s) offered. Eight studies reported abstinence outcomes based on self-reported smoking status, although guidelines recommend the use of biochemically validated outcomes (West 2005). ${ }^{100}$ Only six studies reported biochemically validated smoking cessation (Dey 1999; Kaper 2006; Twardella 2007; Patel 2010; Papadakis 2011; Selby 2014), ${ }^{41-43,53,55,56}$ but Dey $1999^{53}$ was not eligible for inclusion into the meta-analysis for the abstinence comparison because follow-up was terminated at 14 weeks. Fortunately, the two studies that assessed the effect of full financing interventions (Kaper 2006; Twardella 2007) ${ }^{55,56}$ (for which the effect of financial interventions was significant) reported longer term biochemically validated abstinence rates, and hence, have lower risk of bias in this respect. It is possible that reliance on self report could have introduced bias in the rest of the studies, as participants who were benefiting from free treatment might be more likely to give socially desirable answers than those in the control group. From the methodological quality assessment of the economic evaluations, it became clear that only one of the studies reporting on cost effectiveness had performed a full economic evaluation (Kaper 2006). ${ }^{56}$ Results were presented in terms of costs per additional quitter or costs per person enrolled. Only one study examined cost effectiveness in terms of quality-adjusted life years (QALY) saved 
(Kaper 2006). ${ }^{56}$ As a result, no comparisons can be made with economic evaluations of other preventive healthcare treatments.

\section{Agreements and disagreements with other studies or reviews}

This is the only review to assess the effects of financial interventions directed at healthcare providers and smokers to encourage the prescription and use of smoking cessation treatments. We found four reviews (Bains 1998; Hamilton 2013; Cahill 2014; Cahill 2015) ${ }^{28,70,101,102}$ examining the effects of financial interventions, but they included studies that offered a financial benefit for abstinence rather than coverage of the cost of smoking cessation treatment. Bains $1998^{101}$ and Cahill $2015^{28}$ discussed the use and impact of incentives in population-based smoking cessation programs. Smokers participated in contests and lotteries or received financial incentives. Cahill $2014^{102}$ addressed the effectiveness of workplace interventions for smoking cessation. Hamilton $2013^{70}$ examined the effect of providing financial incentives to healthcare professionals on the provision and impact of smoking cessation interventions. They did not find sufficient evidence to show an effect of financial incentives for healthcare professionals on a reduction in smoking rates.

\section{CONCLUSIONS}

\section{Implications for practice}

In this review, covering the full cost to smokers of using smoking cessation treatment increased the number of participants making a quit attempt, the use of smoking cessation treatment, and the number of successful quitters, when compared with no financial coverage. As the majority of the studies were rated at high or unclear risk of bias in three or more domains, and there was variation between the settings, interventions and participants of the included studies, the results should be interpreted cautiously. The differences in self-reported abstinence rate, number of participants making a quit attempt and use of smoking cessation treatments were modest. However, considering the large population of smokers worldwide and the severe health risks of smoking, even modest effects of financial coverage of treatment on smoking cessation could have a substantial effect on public health. The results of this review suggest that full interventions, which cover the cost of pharmacotherapy in combination with behavioral therapy are most promising. This review did not detect an effect of financial interventions directed at healthcare providers on smoking cessation, which suggests that financial interventions may be more beneficial when allocated to smokers directly. However, due to the small number of studies on financial interventions directed at healthcare providers included in this review it may be premature to draw conclusions based on these results.

\section{Implications for research}

More randomized controlled trials should be performed that are comparable with the studies that are included in this review so that future analyses can be stratified by setting, intervention and participants. This is also true for interventions directed at healthcare providers as only three randomized trials are available thus far. More emphasis needs to be put on appropriate reporting of the primary endpoint of smoking cessation, 
particularly biochemical validation and long-term quitting rates (six months or longer). More randomized trials should assess whether financial interventions aimed at healthcare providers can affect the prescribing pattern and uptake of smoking cessation treatments in addition to the smoking behavior of their participants. Furthermore, full economic evaluations need to be performed to aid academics, policy makers and stakeholders alike. To assess the financial impact of healthcare financing interventions for smoking cessation, it is important to determine the cost effectiveness of these interventions more precisely. A full economic evaluation is needed to enable comparison of cost effectiveness with other preventive healthcare treatments. We also recommend use of a standard definition and classification of healthcare financing interventions, particularly those directed at healthcare providers, as this could facilitate future intervention research, between-studies comparison, and rational allocation of resources. 


\section{Summary of findings for the main comparison}

Interventions directed at individuals: full financial coverage compared to no financial coverage for increasing abstinence from smoking

Interventions directed at individuals: full financial coverage compared to no financial coverage for increasing abstinence from smoking

Patient or population: smokers Setting: medical practices, companies, members of a health insurance company, outpatient respirology clinic Intervention: full financial coverage for abstinence from smoking Comparison: no coverage

\begin{tabular}{|c|c|c|c|c|c|c|}
\hline \multirow[t]{2}{*}{ Outcomes } & \multicolumn{2}{|c|}{$\begin{array}{l}\text { Anticipated absolute } \\
\text { effects* }(95 \% \mathrm{Cl})\end{array}$} & \multirow{2}{*}{$\begin{array}{l}\text { Relative } \\
\text { effect }(95 \% \\
\text { Cl) }\end{array}$} & \multirow{2}{*}{$\begin{array}{l}\text { № of } \\
\text { participants } \\
\text { (studies) }\end{array}$} & \multirow{2}{*}{$\begin{array}{l}\text { Quality of } \\
\text { the evidence } \\
\text { (GRADE) }\end{array}$} & \multirow[t]{2}{*}{ Comments } \\
\hline & $\begin{array}{l}\text { Risk with no } \\
\text { coverage }\end{array}$ & $\begin{array}{l}\text { Risk with } \\
\text { full financial } \\
\text { coverage }\end{array}$ & & & & \\
\hline \multirow{2}{*}{$\begin{array}{l}\text { Abstinence } \\
\text { from smoking }\end{array}$} & \multicolumn{2}{|c|}{ Study population } & \multirow{2}{*}{$\begin{array}{l}\text { RR } 1.77 \\
(1.37 \text { to } \\
2.28)\end{array}$} & \multirow[t]{2}{*}{9333 (6 RCTs) } & \multirow{2}{*}{$\begin{array}{l}\bigoplus \bigoplus \bigoplus \bigoplus \\
\text { Moderate }^{1,2}\end{array}$} & \\
\hline & 84 per 1000 & $\begin{array}{l}149 \text { per } 1000 \\
\text { (115 to } 192)\end{array}$ & & & & \\
\hline
\end{tabular}

*The risk in the intervention group (and its 95\% confidence interval) is based on the assumed risk in the comparison group and the relative effect of the intervention (and its $95 \% \mathrm{Cl}$ ). $\mathrm{Cl}$ : Confidence interval; RR: Risk ratio

GRADE Working Group grades of evidence High quality: we are very confident that the true effect lies close to that of the estimate of the effect Moderate quality: we are moderately confident in the effect estimate: the true effect is likely to be close to the estimate of the effect, but there is a possibility that it is substantially different Low quality: our confidence in the effect estimate is limited: the true effect may be substantially different from the estimate of the effect Very low quality: we have very little confidence in the effect estimate: the true effect is likely to be substantially different from the estimate of effect

'Downgraded one level because of risk of bias: all studies except for Hughes 1991 had a serious risk of bias. 2We rated Hughes 1991 and Twardella 2007 serious for imprecision, and Pakhale 2015 very serious for imprecision, however, we did not downgrade the evidence because these studies were small and had a minor effect on the outcome. 


\section{Summary of findings 2 \\ Interventions directed at healthcare providers compared to placebo for increasing the use of smoking cessation treatment}

Interventions directed at healthcare providers compared to no interventions for increasing the use of smoking cessation treatment

Patient or population: physicians and clinics from a multispecialty group practice Setting: health clinics in the USA and group practices in Germany Intervention: financial interventions directed at healthcare providers (pay for performance and direct payment) Comparison: no financial intervention

\begin{tabular}{|c|c|c|c|c|c|c|}
\hline \multirow[t]{2}{*}{ Outcomes } & \multicolumn{2}{|c|}{$\begin{array}{l}\text { Anticipated absolute effects }{ }^{*}(95 \% \\
\mathrm{CI})\end{array}$} & \multirow{2}{*}{$\begin{array}{l}\text { Relative } \\
\text { effect } \\
(95 \% \mathrm{Cl})\end{array}$} & \multirow{2}{*}{$\begin{array}{l}\text { № of } \\
\text { participants } \\
\text { (studies) }\end{array}$} & \multirow{2}{*}{$\begin{array}{l}\text { Quality of } \\
\text { the evidence } \\
\text { (GRADE) }\end{array}$} & \multirow[t]{2}{*}{ Comments } \\
\hline & $\begin{array}{l}\text { Risk with no } \\
\text { interventions }\end{array}$ & $\begin{array}{l}\text { Risk with } \\
\text { interventions } \\
\text { directed at } \\
\text { healthcare } \\
\text { providers }\end{array}$ & & & & \\
\hline \multirow{2}{*}{$\begin{array}{l}\text { Abstinence } \\
\text { from } \\
\text { smoking }\end{array}$} & \multicolumn{2}{|c|}{ Study population } & \multirow{2}{*}{$\begin{array}{l}\text { RR } 1.16 \\
(0.98 \text { to } \\
1.37)\end{array}$} & \multirow[t]{2}{*}{2311 (2 RCTs) } & \multirow{2}{*}{$\begin{array}{l}\oplus \bigoplus \bigoplus \ominus \\
\text { Moderate }^{1}\end{array}$} & \\
\hline & 181 per 1000 & $\begin{array}{l}209 \text { per } 1000(177 \\
\text { to } 247)\end{array}$ & & & & \\
\hline
\end{tabular}
*The risk in the intervention group (and its $95 \%$ confidence interval) is based on the assumed risk in the
comparison group and the relative effect of the intervention (and its $95 \% \mathrm{CI}$ ). Cl: Confidence interval; RR: Risk
ratio
GRADE Working Group grades of evidence High quality: we are very confident that the true effect lies close
to that of the estimate of the effect Moderate quality: we are moderately confident in the effect estimate:
the true effect is likely to be close to the estimate of the effect, but there is a possibility that it is substantially
different Low quality: our confidence in the effect estimate is limited: the true effect may be substantially
different from the estimate of the effect Very low quality: we have very little confidence in the effect estimate:
the true effect is likely to be substantially different from the estimate of effect

'Downgraded one level because both studies were judged to be at serious risk of bias. 


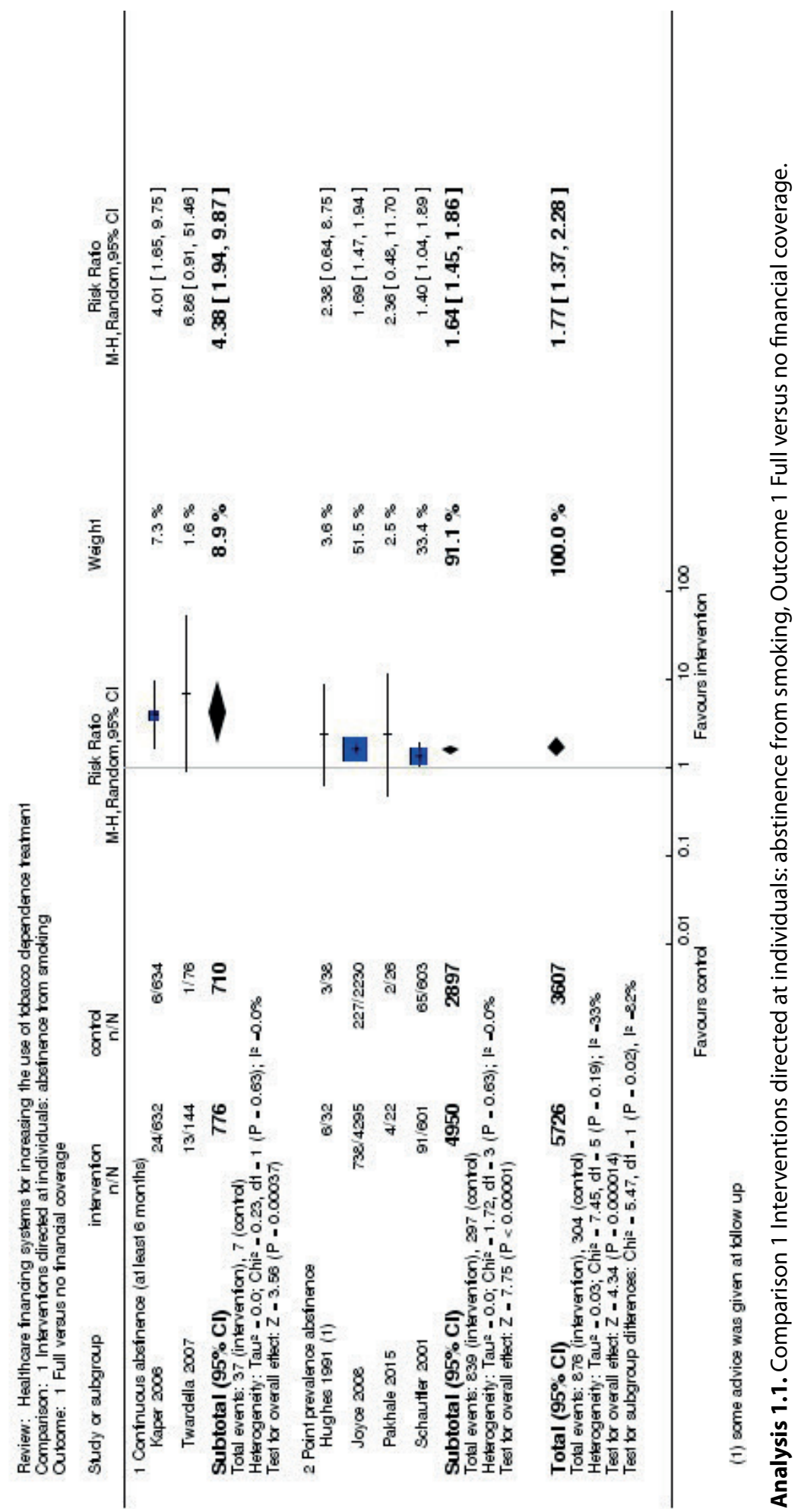




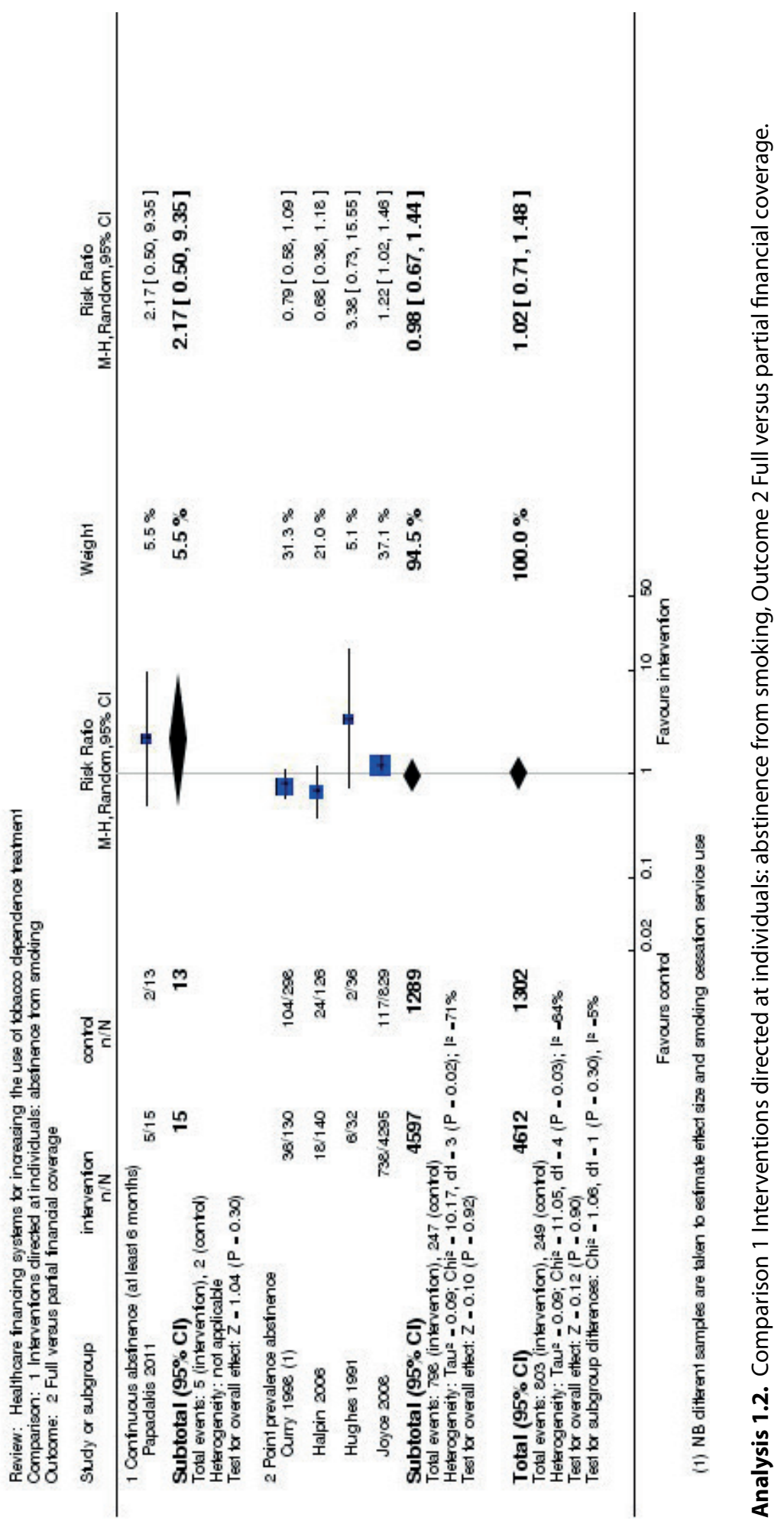




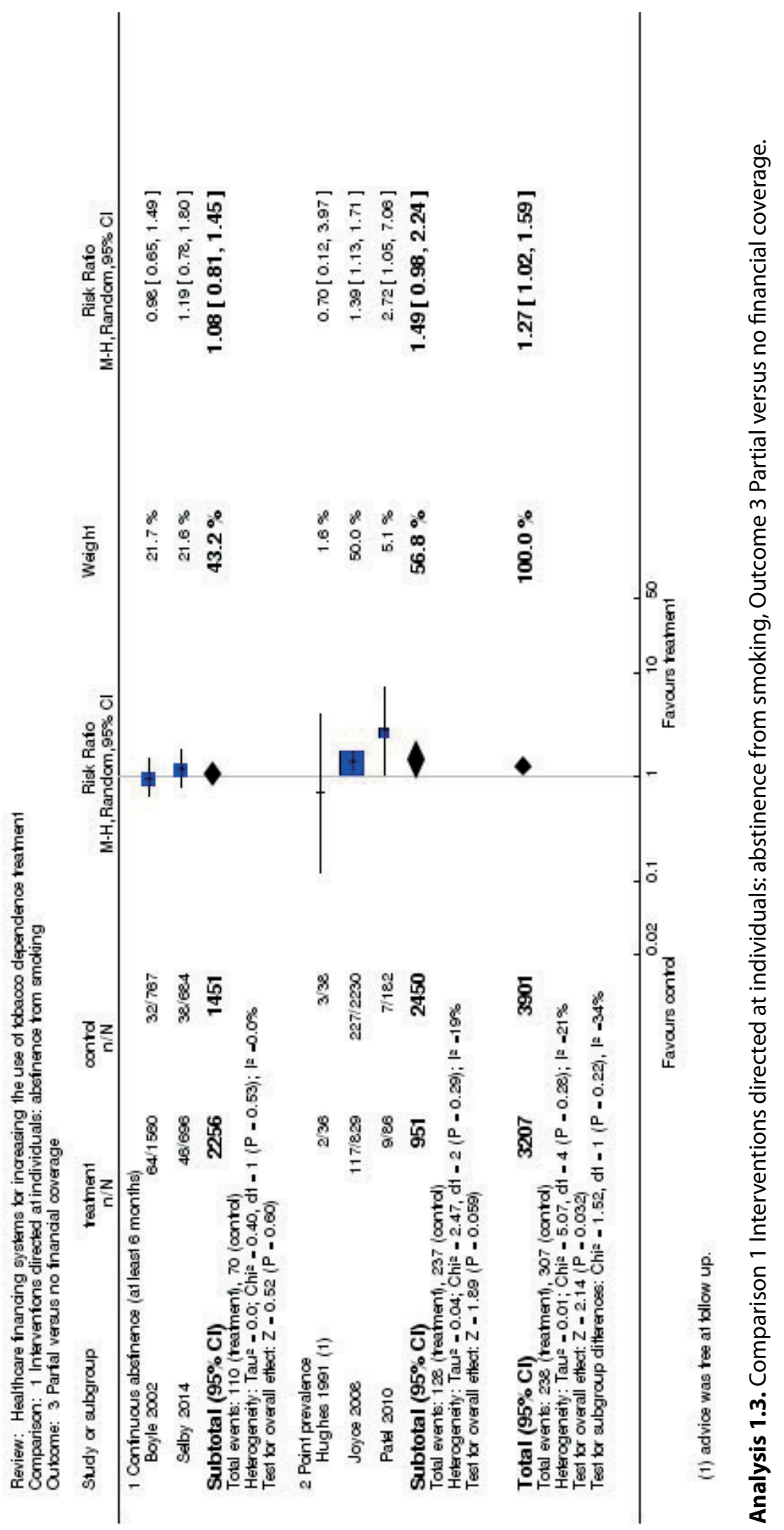




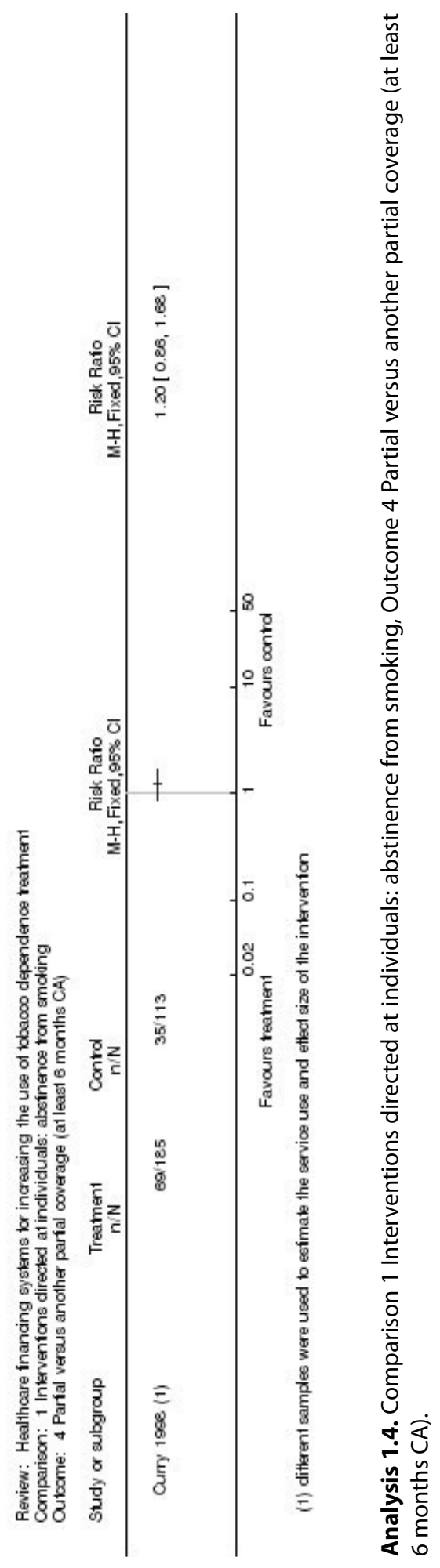




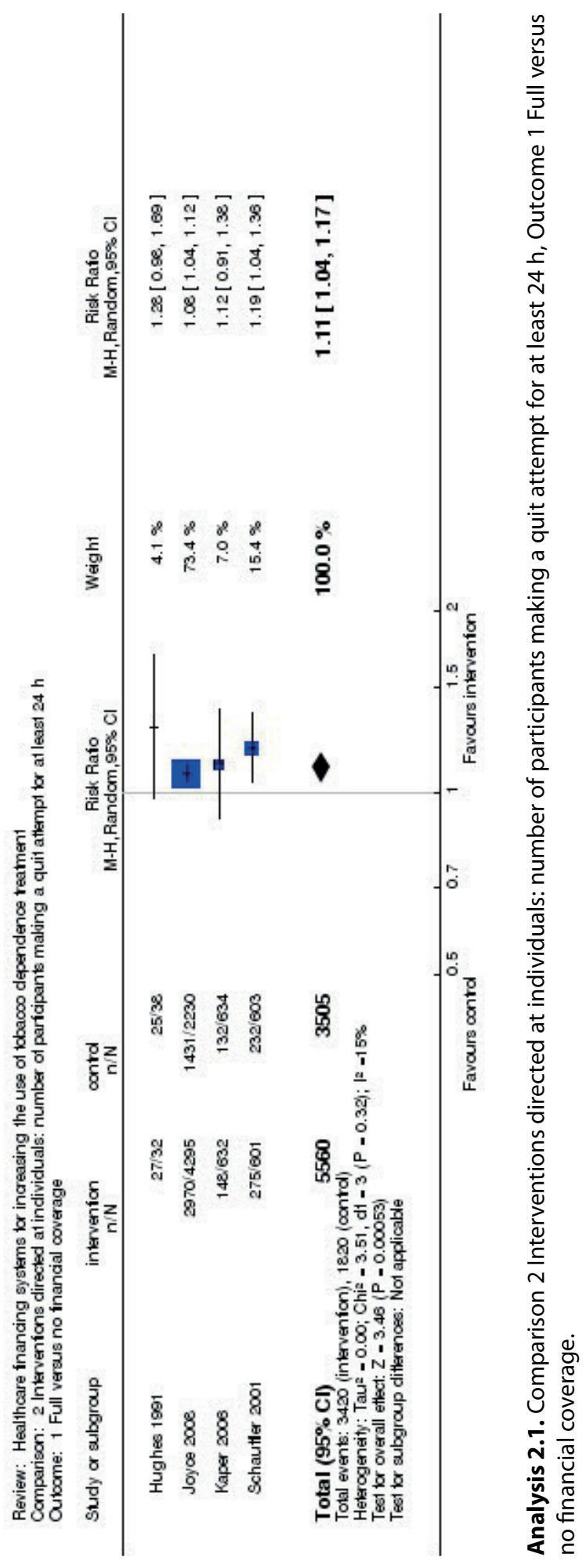




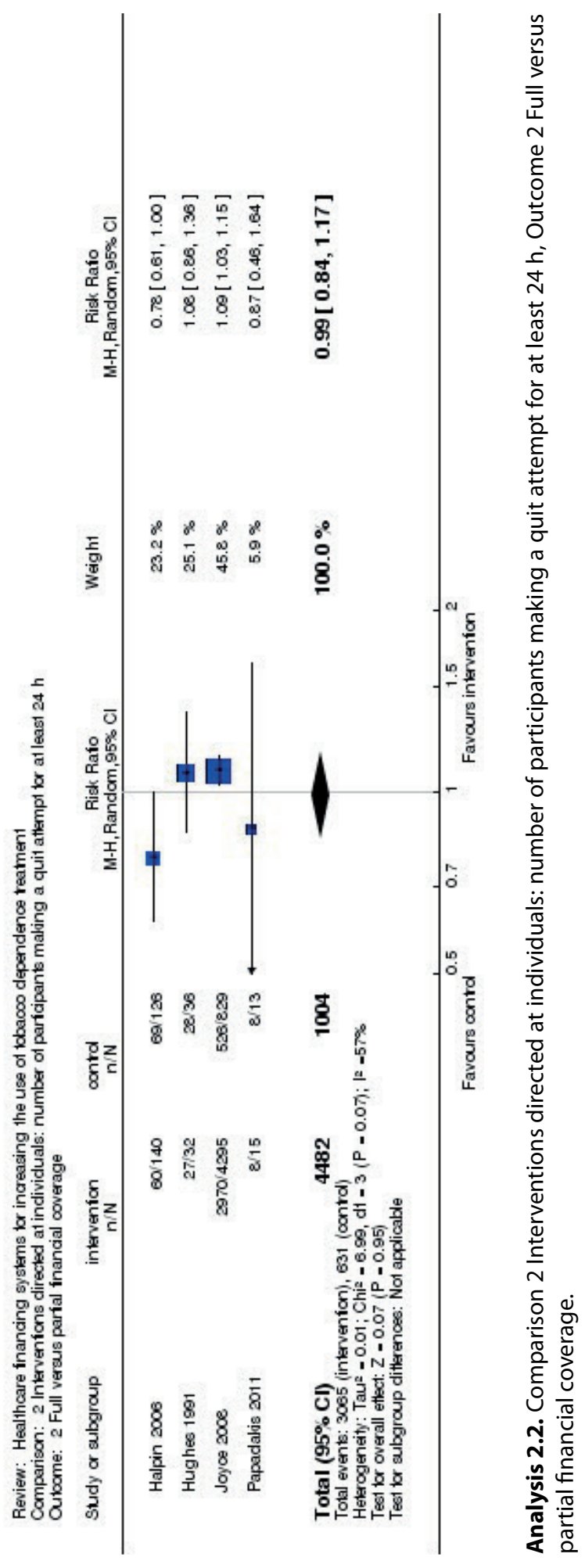




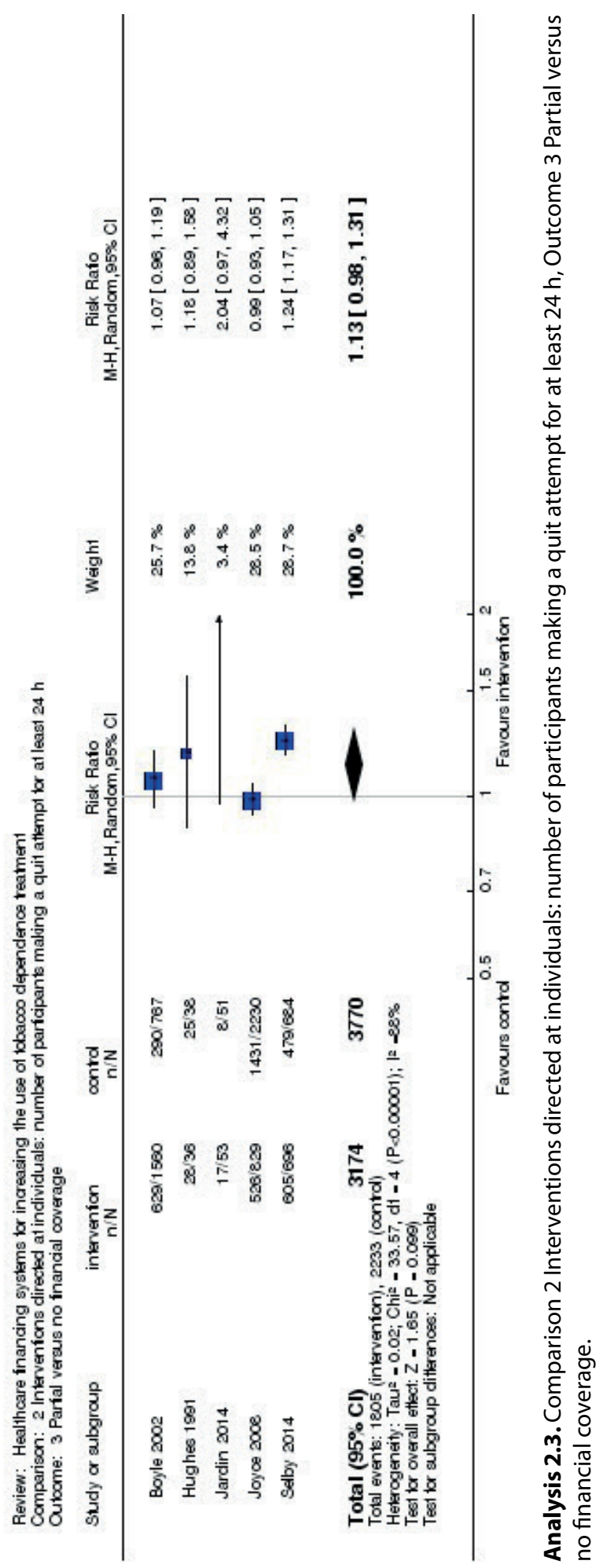




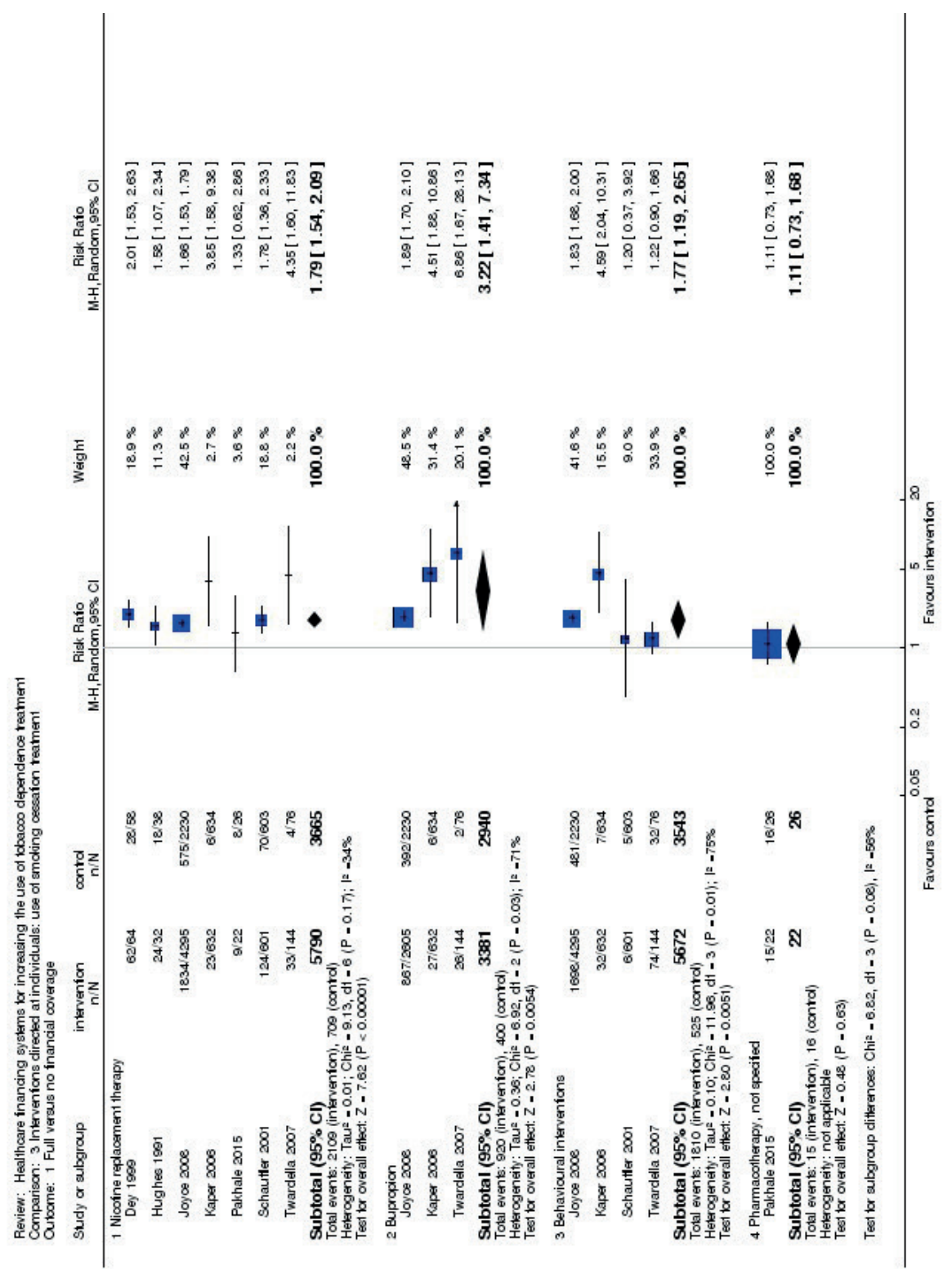

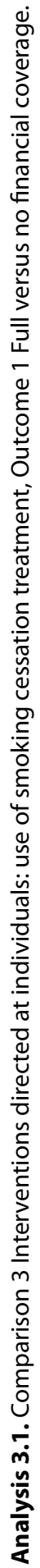




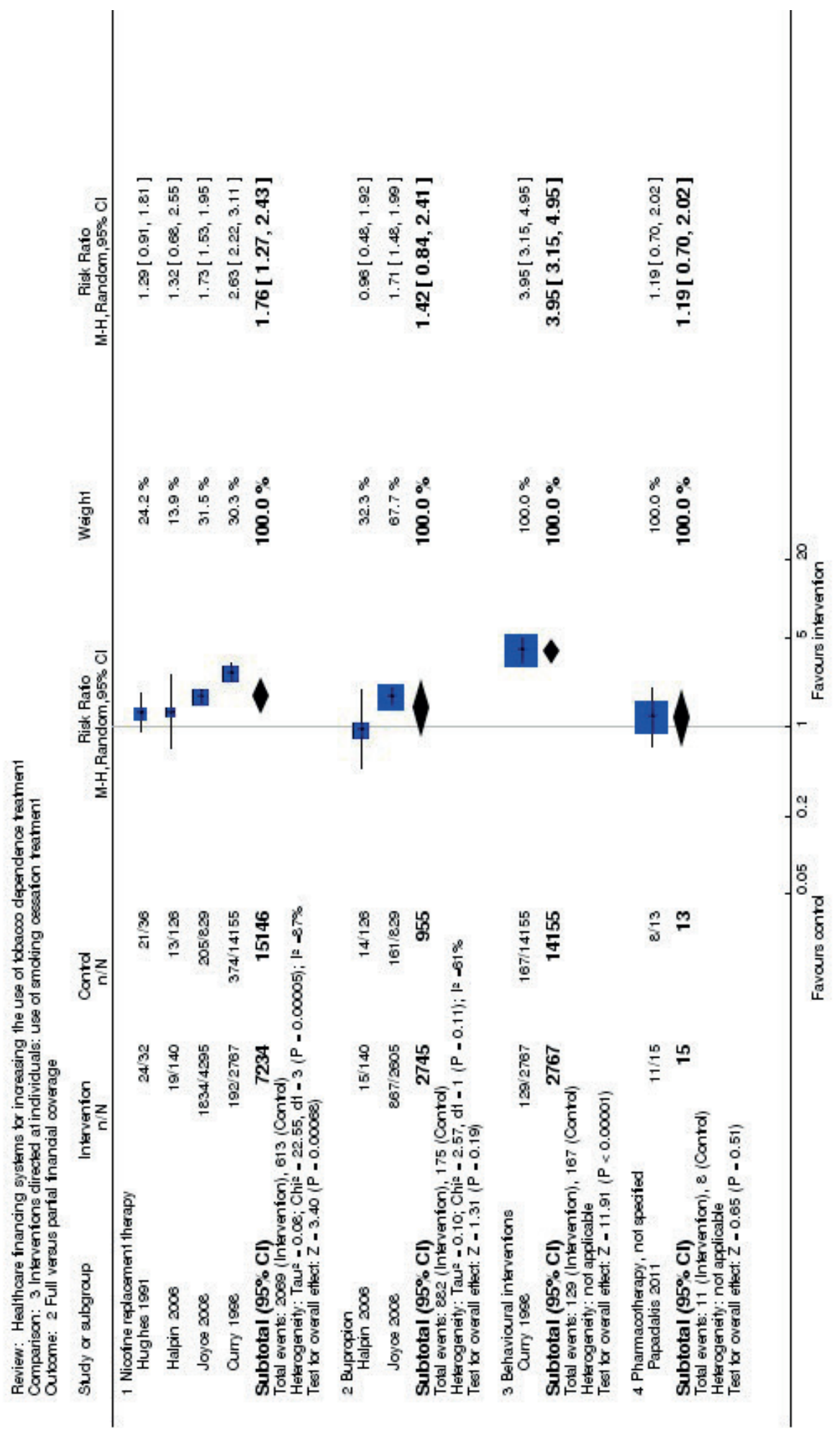

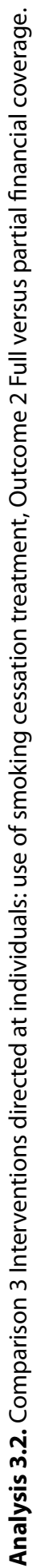




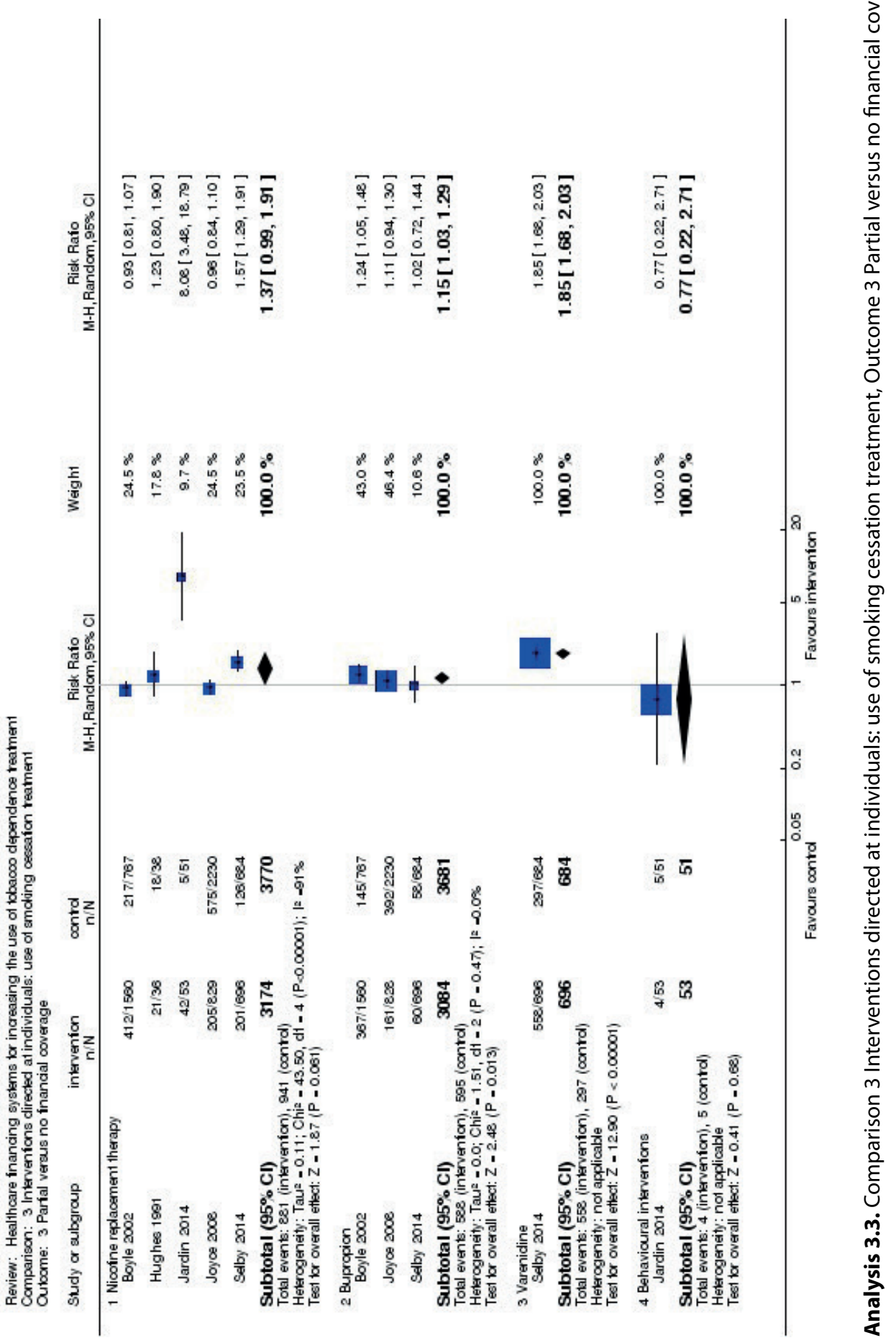




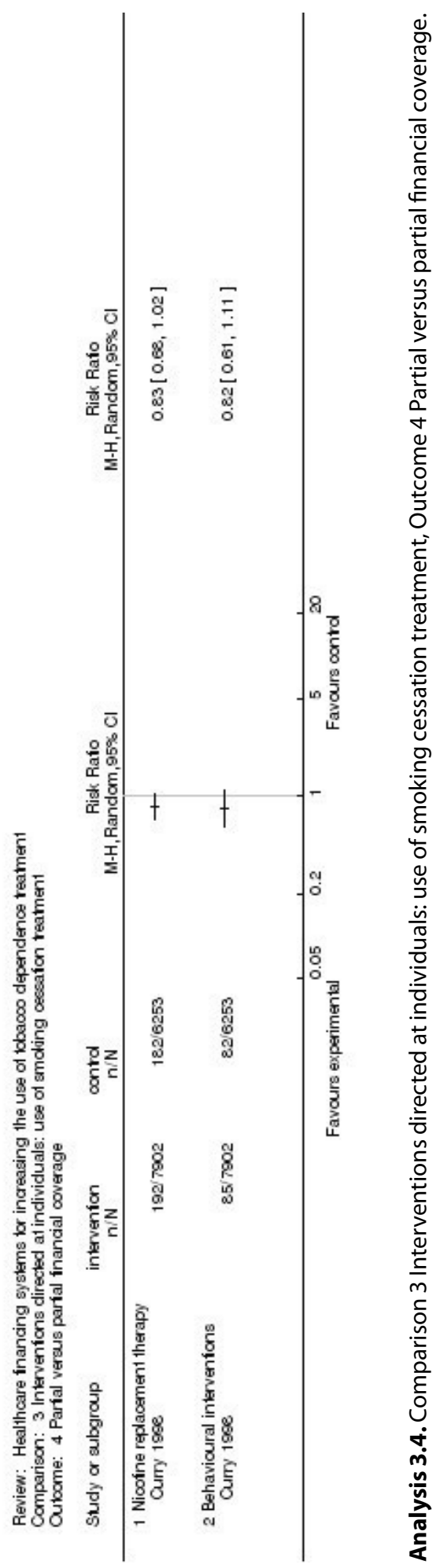




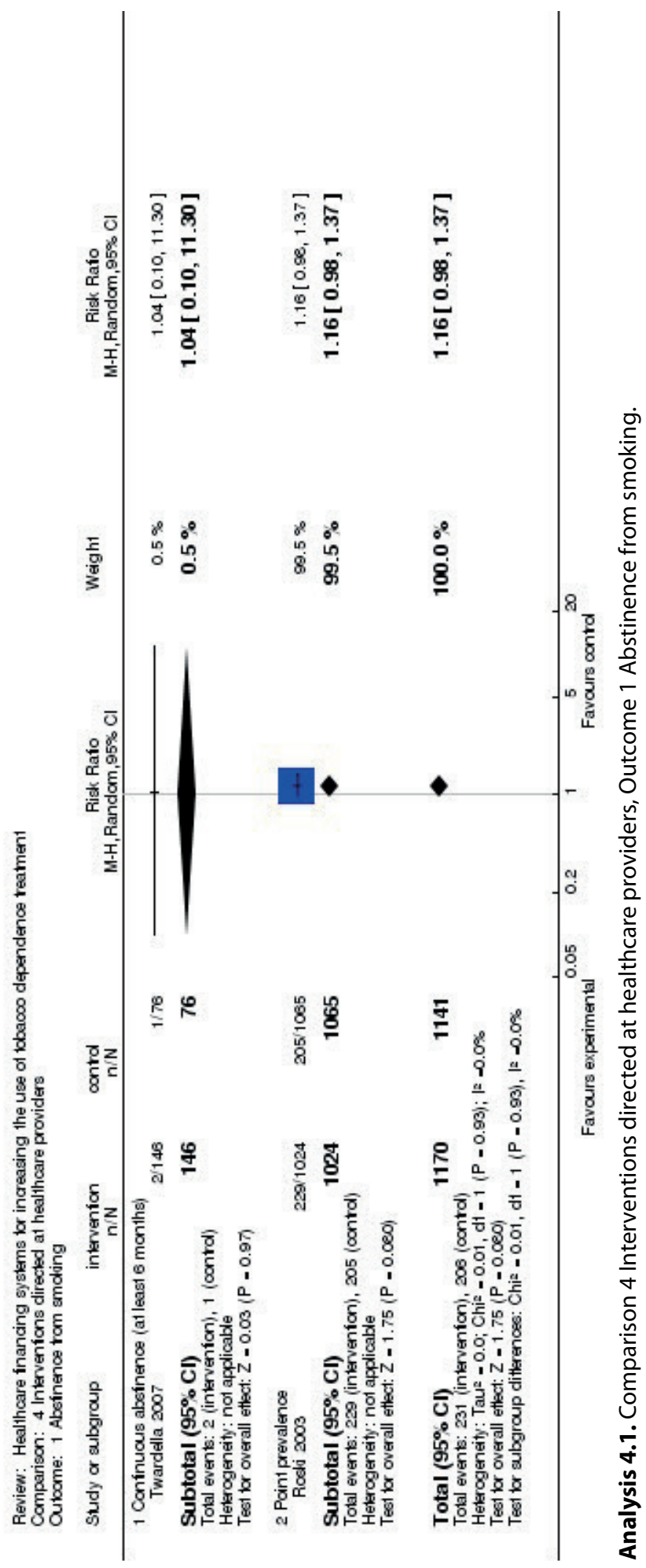




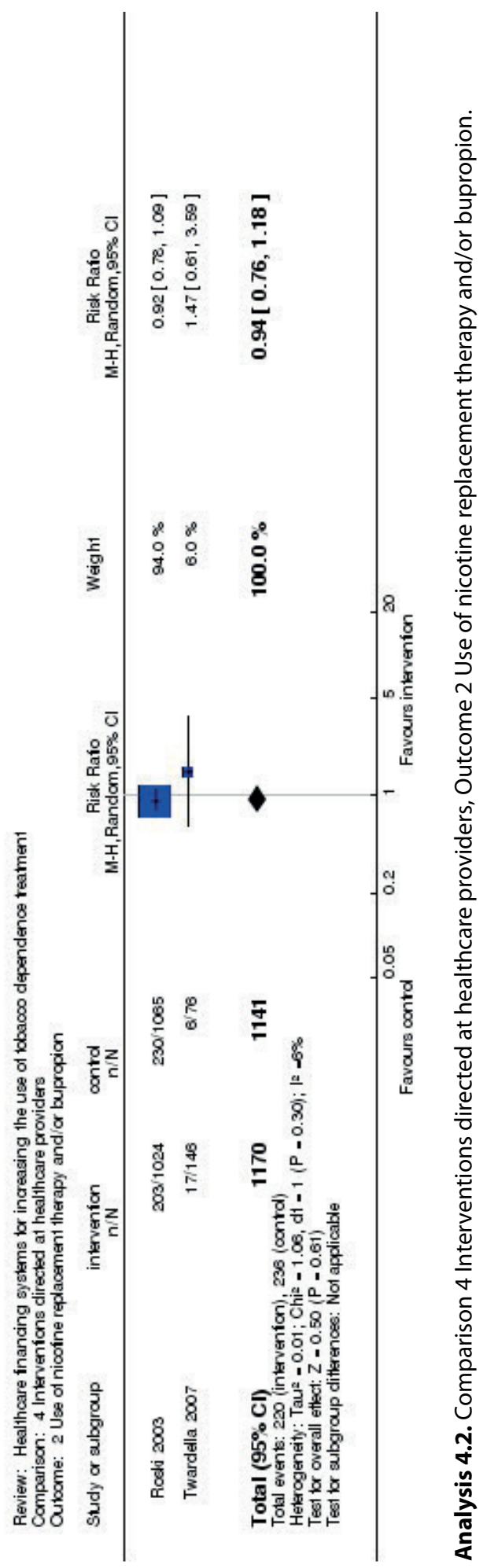




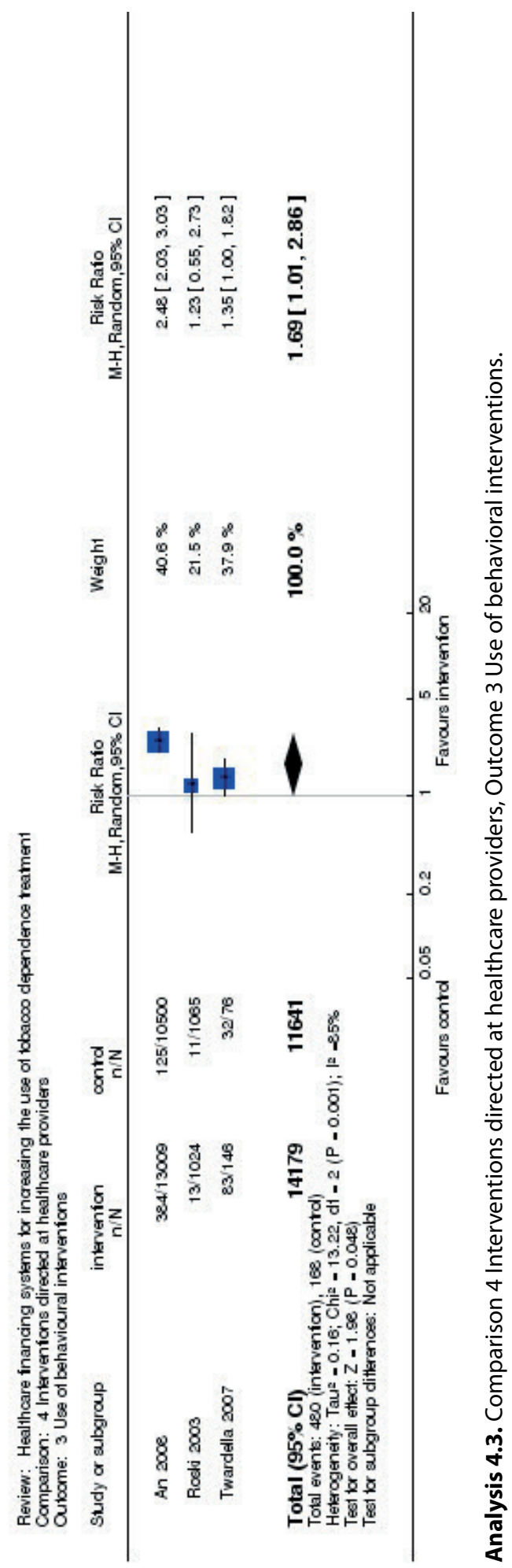




\section{REFERENCES}

1. Aveyard P, West R. Managing smoking cessation. Bmj. 2007;335(7609):37-41.

2. Edwards SA, Bondy SJ, Callaghan RC, Mann RE. Prevalence of unassisted quit attempts in population-based studies: a systematic review of the literature. Addict Behav. 2014;39(3):512519.

3. West R, McNeill A, Raw M. Smoking cessation guidelines for health professionals: an update. Health Education Authority. Thorax. 2000;55(12):987-999.

4. Lancaster T, Stead LF. Individual behavioural counselling for smoking cessation. Cochrane database of systematic reviews. 2017(3).

5. Nides M. Update on pharmacologic options for smoking cessation treatment. Am J Med. 2008;121(4 Suppl 1):S20-31.

6. Stead LF, Carroll AJ, Lancaster T. Group behaviour therapy programmes for smoking cessation. Cochrane database of systematic reviews. 2017(3).

7. Foulds J. The neurobiological basis for partial agonist treatment of nicotine dependence: varenicline. Int J Clin Pract. 2006;60(5):571-576.

8. Mitrouska I, Bouloukaki I, Siafakas NM. Pharmacological approaches to smoking cessation. Pulm Pharmacol Ther. 2007;20(3):220-232.

9. West R, Raw M, McNeill A, et al. Health-care interventions to promote and assist tobacco cessation: a review of efficacy, effectiveness and affordability for use in national guideline development. Addiction. 2015;110(9):1388-1403.

10. Benowitz NL. Neurobiology of nicotine addiction: implications for smoking cessation treatment. Am J Med. 2008;121(4 Suppl 1):S3-10.

11. Coleman T, Wynn AT, Barrett S, Wilson A, Adams S. Intervention study to evaluate pilot health promotion payment aimed at increasing general practitioners' antismoking advice to smokers. BMJ (clinical research ed). 2001;323(7310):435-436.

12. Briesacher BA, Gurwitz JH, Soumerai SB. Patients at-risk for cost-related medication nonadherence: a review of the literature. Journal of general internal medicine. 2007;22(6):864-871.

13. Cox JL, McKenna JP. Nicotine gum: does providing it free in a smoking cessation program alter success rates? Journal of family practice. 1990;31:278-280.

14. Gencarelli D. Medicaid prescription drug coverage: state efforts to control costs. 2003.

15. Goto R, Nishimura S, Ida T. Discrete choice experiment of smoking cessation behaviour in Japan. Tob Control. 2007;16(5):336-343.

16. Hollenbeck BK, Nallamothu BK. Financial incentives and the art of payment reform. Jama. 2011;306(18):2028-2030.

17. Cheung AM, Tsevat J. Economic evaluations of smoking interventions. Prev Med. 1997;26(2):271273.

18. Peckham S, Wallace A. Pay for performance schemes in primary care: what have we learnt? Qual Prim Care. 2010;18(2):111-116.

19. Van Herck P, De Smedt D, Annemans L, Remmen R, Rosenthal MB, Sermeus W. Systematic review: Effects, design choices, and context of pay-for-performance in health care. BMC Health Serv Res. 2010;10:247.

20. Schoen C, Osborn R, Doty MM, Squires D, Peugh J, Applebaum S. A Survey Of Primary Care Physicians In Eleven Countries, 2009: Perspectives On Care, Costs, And Experiences: Doctors say problems exist across all eleven countries, although some nations are doing a better job than others. Health affairs. 2009;28(Suppl1):w1171-w1183. 
21. de Bruin SR, Baan CA, Struijs JN. Pay-for-performance in disease management: a systematic review of the literature. BMC Health Serv Res. 2011;11:272.

22. Flodgren G, Eccles MP, Shepperd S, Scott A, Parmelli E, Beyer FR. An overview of reviews evaluating the effectiveness of financial incentives in changing healthcare professional behaviours and patient outcomes. Cochrane database of systematic reviews. 2011(7).

23. Ryan AM, Krinsky S, Kontopantelis E, Doran T. Long-term evidence for the effect of payfor-performance in primary care on mortality in the UK: a population study. The Lancet. 2016;388(10041):268-274.

24. Steel N, Maisey S, Clark A, Fleetcroft R, Howe A. Quality of clinical primary care and targeted incentive payments: an observational study. Br J Gen Pract. 2007;57(539):449-454.

25. Scott A, Sivey $P$, Ouakrim DA, et al. The effect of financial incentives on the quality of health care provided by primary care physicians. Cochrane database of systematic reviews. 2011(9).

26. Parrott S, Godfrey C. Economics of smoking cessation. Bmj. 2004;328(7445):947-949.

27. Ronckers ET, Groot W, Ament AJ. Systematic review of economic evaluations of smoking cessation: standardizing the cost-effectiveness. Med Decis Making. 2005;25(4):437-448.

28. Cahill K, Hartmann-Boyce J, Perera R. Incentives for smoking cessation. Cochrane Database Syst Rev. 2015(5):Cd004307.

29. Hughes JR, Keely JP, Niaura RS, Ossip-Klein DJ, Richmond RL, Swan GE. Measures of abstinence in clinical trials: issues and recommendations. Nicotine \& Tobacco Research. 2003;5(1):13-25.

30. Verification SSoB. Biochemical verification of tobacco use and cessation. Nicotine \& Tobacco Research. 2002;4(2):149-159.

31. Higgings JA, D. G. . Chapter 8: Assessing risk of bias in included studies. In. Cochrane handbook for systematic reviews of interventions, version 5.1.02011.

32. Review Manager 5 (RevMan 5) [computer program]. Version 5.3. Copenhagen: The Nordic Cochrane Centre 2014.

33. Evers $S$, Goossens $M$, de Vet $H$, van Tulder $M$, Ament A. Criteria list for assessment of methodological quality of economic evaluations: Consensus on Health Economic Criteria. Int $J$ Technol Assess Health Care. 2005;21(2):240-245.

34. Alberg AJ, Stashefsky Margalit R, Burke A, et al. The influence of offering free transdermal nicotine patches on quit rates in a local health department's smoking cessation program. Addict Behav. 2004;29(9):1763-1778.

35. Drummond MF, Sculpher MJ, Claxton K, Stoddart GL, Torrance GW. Methods for the economic evaluation of health care programmes. 4th Edition ed. New York: Oxford university press; 2015.

36. Schünemann HJ, Oxman AD, Higgins JP, Vist GE, Glasziou PG, GH. Chapter 11: Presenting results and 'Summary of findings' tables. In. Cochrane Handbook for Systematic Reviews of Interventions. Version 5.1.0 (updated March 2011)2011.

37. Guyatt GH, Oxman AD, Schunemann HJ, Tugwell P, Knottnerus A. GRADE guidelines: a new series of articles in the Journal of Clinical Epidemiology. J Clin Epidemiol. 2011;64(4):380-382.

38. Moher D, Liberati A, Tetzlaff J, Altman DG. Preferred reporting items for systematic reviews and meta-analyses: the PRISMA statement. PLoS Med. 2009;6(7):e1000097.

39. Jardin $\mathrm{BF}$, Cropsey $\mathrm{KL}$, Wahlquist $\mathrm{AE}$, et al. Evaluating the effect of access to free medication to quit smoking: a clinical trial testing the role of motivation. Nicotine \& tobacco research. 2014;16(7):992-999.

40. Pakhale S, Baron J, Armstrong MA, et al. A pilot randomized controlled trial of smoking cessation in an outpatient respirology clinic. Canadian respiratory journal. 2015;22(2):91-96. 
41. Papadakis S, Aitken D, Gocan S, Riley D, Laplante MA, Bhatnagar-Bost A. A randomised controlled pilot study of standardised counselling and cost-free pharmacotherapy for smoking cessation among stroke and TIA patients. BMJ open. 2011;1(2):e000366.

42. Patel V, Shaw JW, Leischow SJ, Ranger-Moore J, Muramoto M. Effect of nicotine gum price on medication acquisition and smoking cessation in an over-the-counter setting. Value in health. 2010;13(3):A197.

43. Selby P, Brosky G, Oh P, Raymond V, Arteaga C, Ranger S. A pragmatic, randomized, controlled study evaluating the impact of access to smoking cessation pharmacotherapy coverage on the proportion of successful quitters in a Canadian population of smokers motivated to quit (ACCESSATION). BMC public health. 2014;14:433.

44. Willemsen MC, Segaar D, van Schayck OC. Population impact of reimbursement for smoking cessation: a natural experiment in The Netherlands. Addiction. 2013;108(3):602-604.

45. An LC, Bluhm JH, Foldes SS, et al. A randomized trial of a pay-for-performance program targeting clinician referral to a state tobacco quitline. Archives ofinternal medicine. 2008;168(18):1993-1999.

46. Boyle RG, Solberg LI, Magnan S, Davidson G, Alesci NL. Does insurance coverage for drug therapy affect smoking cessation? Health Affairs. 2002;21(6):162-168.

47. Curry SJ, Grothaus LC, McAfee T, Pabiniak C. Use and cost effectiveness of smoking-cessation services under four insurance plans in a health maintenance organization. New england journal of medicine. 1998;339(10):673-679.

48. Halpin HA, McMenamin SB, Rideout J, Boyce-Smith G. The costs and effectiveness of different benefit designs for treating tobacco dependence: results from a randomized trial. Inquiry. 2006;43(1):54-65.

49. Hughes JR, Wadland WC, Fenwick JW, Lewis J, Bickel WK. Effect of cost on the self-administration and efficacy of nicotine gum: a preliminary study. Preventive medicine. 1991;20(4):486-496.

50. Joyce GF, Niaura R, Maglione M, et al. The effectiveness of covering smoking cessation services for medicare beneficiaries. Health services research. 2008;43(6):2106-2123.

51. Roski J, Jeddeloh R, An L, et al. The impact of financial incentives and a patient registry on preventive care quality: increasing provider adherence to evidence-based smoking cessation practice guidelines. Preventive medicine. 2003;36(3):291-299.

52. Schauffler HH, McMenamin S, Olson K, Boyce-Smith G, Rideout JA, Kamil J. Variations in treatment benefits influence smoking cessation: results of a randomised controlled trial. Tobacco control. 2001;10(2):175-180.

53. Dey P, Foy R, Woodman M, Fullard B, Gibbs A. Should smoking cessation cost a packet? A pilot randomized controlled trial of the cost-effectiveness of distributing nicotine therapy free of charge. British journal of general practice. 1999;49(439):127-128.

54. Kaper J, Wagena EJ, van Schayck CP, Severens JL. Encouraging smokers to quit: the cost effectiveness of reimbursing the costs of smoking cessation treatment. Pharmacoeconomics. 2006;24(5):453-464.

55. Twardella $D$, Brenner $H$. Effects of practitioner education, practitioner payment and reimbursement of patients' drug costs on smoking cessation in primary care: a cluster randomised trial. Tobacco control. 2007;16(1):15-21.

56. Kaper J, Wagena EJ, van Schayck CP, Severens JL. Encouraging smokers to quit: the cost effectiveness of reimbursing the costs of smoking cessation treatment. Pharmacoeconomics. 2006;24(5):453-464. 
57. Kaper J, Wagena EJ, Willemsen MC, van Schayck CP. Reimbursement for smoking cessation treatment may double the abstinence rate: results of a randomized trial. Addiction. 2005;100(7):1012-1020.

58. Bonevski B, Guillaumier A, Shakeshaft A, et al. An organisational change intervention for increasing the delivery of smoking cessation support in addiction treatment centres: study protocol for a randomized controlled trial. Trials. 2016;17(1):290.

59. Courtney RJ, Bradford D, Martire KA, et al. A randomized clinical trial of a financial education intervention with nicotine replacement therapy (NRT) for low socio-economic status Australian smokers: a study protocol. Addiction (abingdon, england). 2014;109(10):1602-1611.

60. Härter M, Bartsch AL, Egger N, et al. Evaluating a collaborative smoking cessation intervention in primary care (ENTER): study protocol for a cluster-randomized controlled trial. Trials. 2015; 16:447.

61. Ostroff JS, Li Y, Shelley DR. Dentists United to Extinguish Tobacco (DUET): a study protocol for a cluster randomized, controlled trial for enhancing implementation of clinical practice guidelines for treating tobacco dependence in dental care settings. Implementation science. 2014;9:25.

62. Park ER, Ostroff JS, Perez GK, et al. Integrating tobacco treatment into cancer care: study protocol for a randomized controlled comparative effectiveness trial. Contemporary clinical trials. 2016;50:54-65.

63. NCT00962988. Efficacy and Cost-Effectiveness of Cost-free Pharmacotherapy for Smoking Cessation for High-risk Smokers With Cerebrovascular Disease. In. Canada, Ontario2009.

64. Cummings KM, Fix B, Celestino P, Carlin-Menter S, O'Connor R, Hyland A. Reach, efficacy, and cost-effectiveness of free nicotine medication giveaway programs. Journal of Public Health Management and Practice. 2006;12(1):37-43.

65. Oswald JS, Worden WL, Cox JL. The efficacy of nicotine gum in group-centered smoking cessation therapy in a family practice. J Fam Pract. 1988;27(2):179-183.

66. Parnes B, Main DS, Holcomb S, Pace W. Tobacco cessation counseling among underserved patients: a report from CaReNet. J Fam Pract. 2002;51(1):65-69.

67. Russos S, Keating K, Hovell MF, et al. Counseling youth in tobacco-use prevention: determinants of clinician compliance. Preventive medicine. 1999;29(1):13-21.

68. Stone TT, Longo DR, Phillips JR, Hewett JE, Riley SL. Health care system and insurer support for smoking cessation guideline implementation. Journal of health care finance. 2002;29(2):78-86.

69. Bailey SR, Hoopes MJ, Marino M, et al. Effect of Gaining Insurance Coverage on Smoking Cessation in Community Health Centers: A Cohort Study. J Gen Intern Med. 2016;31(10):11981205.

70. Hamilton FL, Greaves F, Majeed A, Millett C. Effectiveness of providing financial incentives to healthcare professionals for smoking cessation activities: systematic review. Tob Control. 2013;22(1):3-8.

71. Amundson G, Solberg LI, Reed M, Martini EM, Carlson R. Paying for quality improvement: compliance with tobacco cessation guidelines. The Joint Commission Journal on Quality and Safety. 2003;29(2):59-65.

72. Andrews $M Z$, Korberly $B$, Schensky $A E$, Baker T. Helping Wisconsin women quit smoking: a successful collaboration. Wisconsin Medical Journal. 2000:68-72.

73. Chang FC, Hu TW, Lin M, Yu PT, Chao KY. Effects of financing smoking cessation outpatient services in Taiwan. Tob Control. 2008;17(3):183-189. 
74. Doescher MP, Whinston MA, Goo A, Cummings D, Huntington J, Saver BG. Pilot study of enhanced tobacco-cessation services coverage for low-income smokers. Nicotine \& tobacco research. 2002;4(Suppl_1):S19-S24.

75. Latts LM, Prochazka AV, Salas NM, Young DA. Smoking cessation in pregnancy: failure of an $\mathrm{HMO}$ pilot project to improve guideline implementation. Nicotine \& tobacco research. 2002;4(Suppl_1):S25-S30.

76. Ringen K, Anderson N, McAfee T, Zbikowski SM, Fales D. Smoking cessation in a blue-collar population: results from an evidence-based pilot program. American journal of industrial medicine. 2002;42(5):367-377.

77. Solberg LI, Davidson G, Alesci NL, Boyle RG, Magnan S. Physician smoking-cessation actions: are they dependent on insurance coverage or on patients? Am J Prev Med. 2002;23(3):160-165.

78. Weisman SR, Hennrikus D, Choi K, et al. Influencing Taft-Hartley Funds to provide tobacco cessation benefits. Am J Prev Med. 2012;43(5 Suppl 3):S237-241.

79. Land T, Warner D, Paskowsky $M$, et al. Medicaid coverage for tobacco dependence treatments in Massachusetts and associated decreases in smoking prevalence. PLoS One. 2010;5(3):e9770.

80. Lave JR, Ives DG, Traven ND, Kuller LH. Evaluation of a health promotion demonstration program for the rural elderly. Health services research. 1996;31(3):261-281.

81. Curry SJ, Wagner EH, Grothaus LC. Evaluation of intrinsic and extrinsic motivation interventions with a self-help smoking cessation program. Journal of consulting and clinical psychology. 1991;59(2):318-324.

82. Donatelle RJ, Prows SL, Champeau D, Hudson D. Randomised controlled trial using social support and financial incentives for high risk pregnant smokers: significant other supporter (SOS) program. Tobacco control. 2000;9 Suppl 3:II167-69.

83. Hovell MF, Slymen DJ, Jones JA, et al. An adolescent tobacco-use prevention trial in orthodontic offices. American journal of public health. 1996;86(12):1760-1766.

84. Kruse GR, Chang Y, Kelley JH, Linder JA, Einbinder JS, Rigotti NA. Healthcare system effects of pay-for-performance for smoking status documentation. Am J Manag Care. 2013;19(7):554-561.

85. McLeod H, Blissett D, Wyatt $S$, Mohammed MA. Effect of pay-for-outcomes and encouraging new providers on national health service smoking cessation services in England: a cluster controlled study. PloS one. 2015;10(4):e0123349.

86. Moskowitz JM, McDonnell DD, Kazinets G, Lee HJ. Online smoking cessation program for Korean Americans: randomized trial to test effects of incentives for program completion and interim surveys. Preventive medicine. 2016;86:70-76.

87. Pardell H, Faixedas M, Salto E, Valverde A, Tresserras TJ, Taberner J. Influence of an economical incentive on smoking cessation at community level. Paper presented at: Society for Research on Nicotine and Tobacco 5th European Meeting November2003.

88. Volpp KG, Gurmankin Levy A, Asch DA, et al. A randomized controlled trial of financial incentives for smoking cessation. Cancer epidemiology, biomarkers \& prevention. 2006;15(1):12-18.

89. Krist $\mathrm{AH}$, Woolf $\mathrm{SH}$, Johnson $\mathrm{RE}$, et al. Patient costs as a barrier to intensive health behavior counseling. Am J Prev Med. 2010;38(3):344-348.

90. Hays JT, Croghan IT, Schroeder DR, et al. Over-the-counter nicotine patch therapy for smoking cessation: results from randomized, double-blind, placebo-controlled, and open label trials. American journal of public health. 1999;89(11):1701-1707.

91. Hockenberry JM, Curry SJ, Fishman PA, et al. Healthcare costs around the time of smoking cessation. American journal of preventive medicine. 2012;42(6):596-601. 
92. Shaw JW, Muramoto M, Moore JRH, W. C., Leischow SJ. A conceptual model of the effects of nicotine gum price on self medication and cigarette consumption in an over the-counter setting. . Society for Research on Nicotine and Tobacco 5th European Meeting 2003; Padua.

93. Amemori $M$, Virtanen J, Korhonen $\mathrm{T}$, Kinnunen $\mathrm{TH}$, Murtomaa H. Impact of educational intervention on implementation of tobacco counselling among oral health professionals: a cluster-randomized community trial. Community dentistry and oral epidemiology. 2013;41(2):120-129.

94. Bardach NS, Wang JJ, De Leon SF, et al. Effect of pay-for-performance incentives on quality of care in small practices with electronic health records: a randomized trial. JAMA. 2013;310(10):1051-1059.

95. Verbiest ME, Chavannes NH, Crone MR, et al. An increase in primary care prescriptions of stopsmoking medication as a result of health insurance coverage in the Netherlands: population based study. Addiction. 2013;108(12):2183-2192.

96. Walsh MM, Belek M, Prakash $P$, et al. The effect of training on the use of tobacco-use cessation guidelines in dental settings. Journal of the American Dental Association (1939). 2012;143(6):602-613.

97. Fu SS, van Ryn M, Nelson D, et al. Proactive tobacco treatment offering free nicotine replacement therapy and telephone counselling for socioeconomically disadvantaged smokers: a randomised clinical trial. Thorax. 2016;71(5):446-453.

98. Kaper J, Wagena EJ, Willemsen MC, van Schayck CP. A randomized controlled trial to assess the effects of reimbursing the costs of smoking cessation therapy on sustained abstinence. Addiction (abingdon, england). 2006;101(11):1656-1661.

99. Hall SM, Humfleet GL, Reus VI, Munoz RF, Hartz DT, Maude-Griffin R. Psychological intervention and antidepressant treatment in smoking cessation. Archives of General Psychiatry. 2002;59(10):930-936.

100. West R, Hajek P, Stead L, Stapleton J. Outcome criteria in smoking cessation trials: proposal for a common standard. Addiction. 2005;100(3):299-303.

101. Bains N, Pickett W, Hoey J. The use and impact of incentives in population-based smoking cessation programs: a review. Am J Health Promot. 1998;12(5):307-320.

102. Cahill K, Lancaster T. Workplace interventions for smoking cessation. Cochrane database of systematic reviews. 2014(2). 


\section{APPENDICES}

Appendix 1. Specialised Register Search Strategy

Search strategy used for Specialised Register (using Cochrane Register of Studies (CRS) software)

\#1 MeSH DESCRIPTOR Insurance Explode All

\#2 MeSH DESCRIPTOR Insurance Coverage Explode All

\#3 MeSH DESCRIPTOR Insurance, Health Explode All

\#4 MeSH DESCRIPTOR Reimbursement Mechanisms Explode All

\#5 MeSH DESCRIPTOR Insurance, Health, Reimbursement Explode All

\#6 MeSH DESCRIPTOR social control policies Explode All

\#7 MeSH DESCRIPTOR health care costs Explode All

\#8 MeSH DESCRIPTOR Quality of Health Care Explode All

\#9 MeSH DESCRIPTOR Fee-for-Service Plans Explode All

\#10 MeSH DESCRIPTOR Physician Incentive Plans Explode All

\#11 MeSH DESCRIPTOR Costs and Cost Analysis Explode All

\#12 MeSH DESCRIPTOR Cost-Benefit Analysis Explode All

\#13 health care costs

\#14 health insurance

\#15 coverage ${ }^{*}: A B, T I$

\#16 reimburse*

\#17 payment*

\#18 remunerat*

\#19 incentive*

\#20 salary or salaries 
\#21 fee or fees

\#22 deductible*

\#23 co?insurance

\#24 co?payment

\#25 capita*

\#26 fund?hold*

\#27 prepay or prepaid

\#28 financ* NEAR incentive*:AB,TI

\#29 cost? NEAR (shar* or free or no):TI,AB

\#30 \#1 OR \#2 OR \#3 OR \#4 OR \#5 OR \#6 OR \#7 OR \#8 OR \#9 OR \#10 OR \#11 OR \#12 OR \#13 OR \#14 OR \#15 OR \#16 OR \#17 OR \#18 OR \#19 OR \#20 OR \#21 OR \#22 OR \#23 OR \#24 OR \#25 OR \#26 OR \#27 or \#28 


\section{Appendix 2. MEDLINE search strategy}

The following topic related terms were combined with $\mathrm{MeSH}$ and free text terms concerning smoking and tobacco use, and with terms to identify trials and other evaluations of healthcare effects used by Cochrane Tobacco Addiction for regular searches of MEDLINE. (See Specialized Register section of Tobacco Addiction Group Module). The free text term 'time series' was included in the trials identification set. exp Insurance, Health, Reimbursement/ or exp Insurance/ or exp Insurance Coverage/ or exp Insurance, Health/ or exp Reimbursement Mechanisms/ or exp Insurance, Health, Reimbursement/ or exp social control policies/ or exp health care costs/ or "Quality of Health Care"/ec or exp Fee-for-Service Plans/ or exp Managed Care Programs/ or exp Physician Incentive Plans/ or exp Employee Incentive Plans/ or (coverage or reimburs\$ or target\$ or payment\$ or remunerat\$ or incentive\$ or financ\$ or salar\$ or fee or fees or deductible\$ or coinsurance or copayment or capita\$ or cost\$ or payment\$ or fundhold\$ or prepay\$ or prepaid).mp. [mp=title, original title, abstract, name of substance word, subject heading word] 


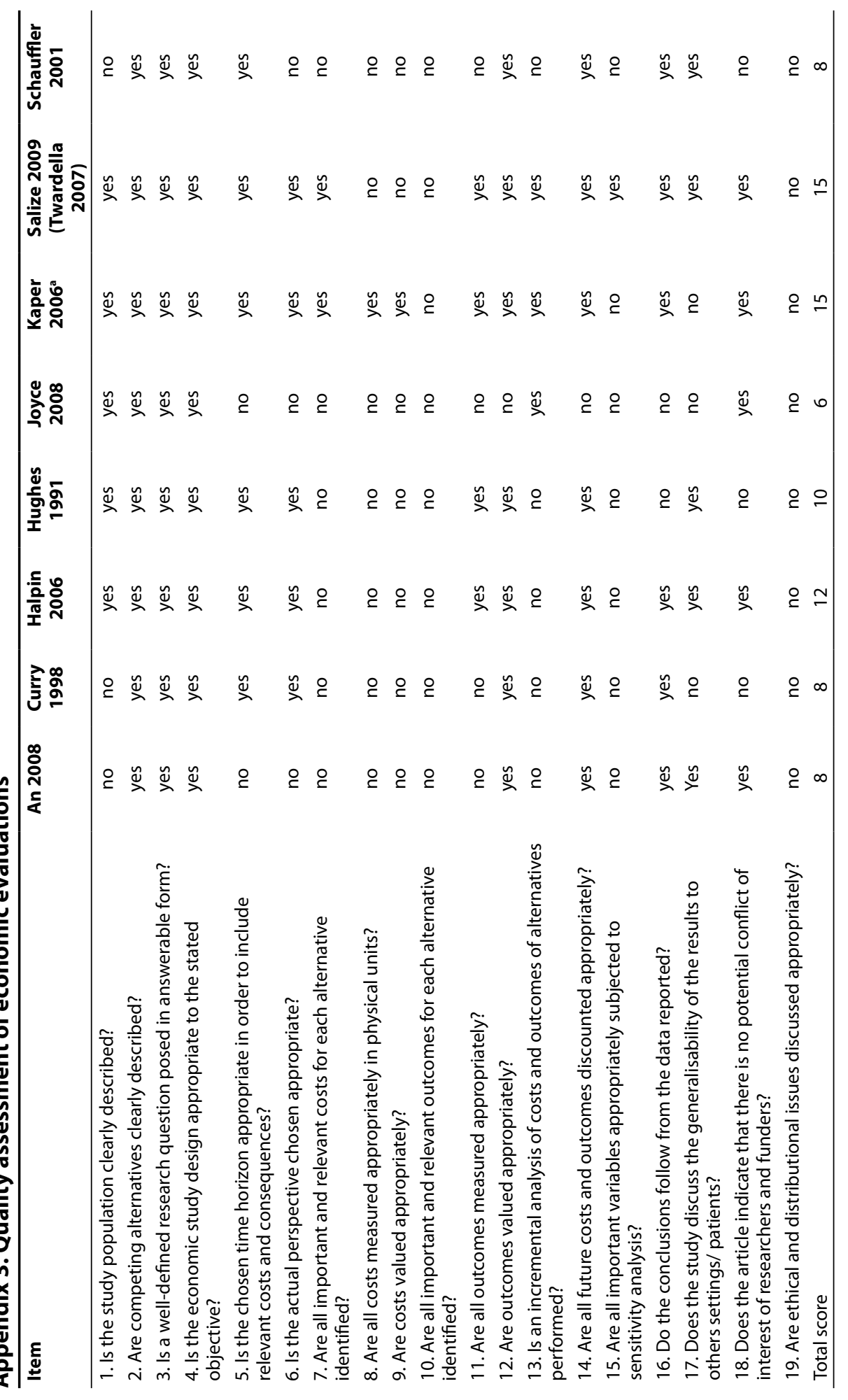




\section{CHAPTER 3}

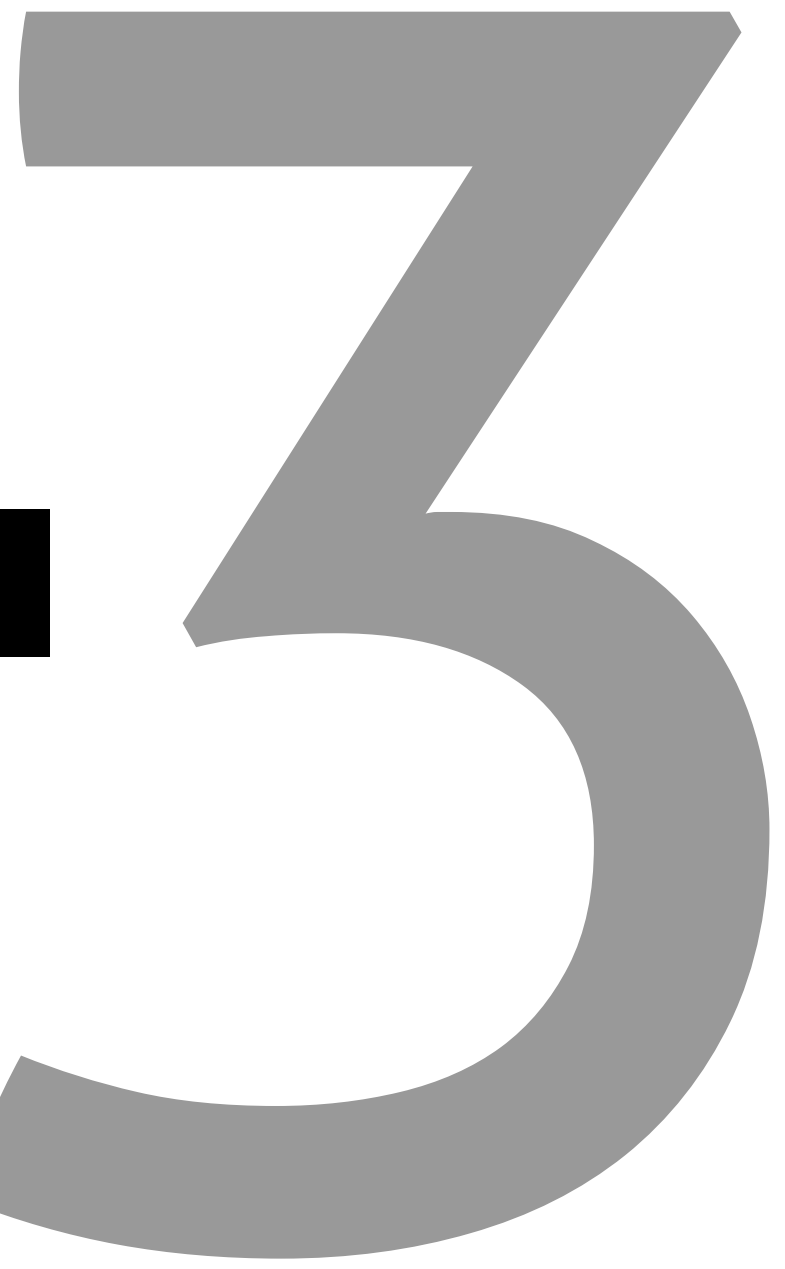




\section{Does free or lower cost smoking cessation medication stimulate quitting? Findings from the International Tobacco Control (ITC) Netherlands and United Kingdom Surveys}

Van den Brand FA, Nagelhout GE, Hummel K, Willemsen MC, McNeill A, van Schayck OCP. Does free or lower cost smoking cessation medication stimulate quitting? Findings from the International Tobacco Control (ITC) Netherlands and UK Surveys. 


\section{ABSTRACT}

Objective: To investigate whether mentioning free or lower cost smoking cessation medication as a trigger for thinking about quitting is related to higher medication use, more quit attempts and quit success, and whether these associations are modified by education and income.

Methods: Data were derived from the 2013 and 2014 surveys of the International Tobacco Control (ITC) Netherlands ( $n=1164)$ and United Kingdom $(n=768)$ cohort. Logistic regression analyses were used to assess associations between mentioning in 2013 that free/lower cost smoking cessation medication was a trigger for thinking about quitting smoking and the use of medication, quit attempts and smoking cessation in 2014.

Results: $37.0 \%$ of smokers in the UK and $24.9 \%$ of smokers in the Netherlands mentioned free/lower cost medication as a trigger for thinking about quitting. Smokers who mentioned this trigger were more likely to have used cessation medication during a quit attempt both in the UK $(O R=4.19, p<0.001)$ and in the Netherlands $(O R=2.14, p=0.033)$. The association between mentioning free/lower cost medication as a trigger for thinking about quitting and actual quit attempts was significant in the UK $(O R=1.45, p=0.030)$, but not in the Netherlands $(O R=1.10, p=0.587)$. There was no significant association with quit success. Associations did not differ across income and education groups.

Conclusion: Free/lower cost smoking cessation medication may increase the use of cessation medication and stimulate quit attempts among smokers with low, moderate, and high education and income. 


\section{INTRODUCTION}

In Western countries, the proportion of smokers is not equally divided among low and high socioeconomic groups. ${ }^{1-3}$ Individuals with lower education and income are more likely to smoke and smoke more cigarettes a day than higher educated and more affluent people. ${ }^{4,5}$ This disparity causes smoking to be the largest contributor to socioeconomic differences in health and mortality observed in Western countries. ${ }^{6,7}$ Although the proportion of people who smoke is generally declining, inequalities according to socioeconomic status (SES) have sustained or increased over time. ${ }^{2,3,8-11}$ In the United Kingdom (UK), $21 \%$ of individuals with no formal qualifications smoke compared to $9 \%$ of individuals with a degree. ${ }^{12}$ In the Netherlands (NL), a comparable SES gap in smoking prevalence exists: $28 \%$ of lower educated adults smoke compared to $19 \%$ of higher educated adults. ${ }^{13}$ In order to reduce this socioeconomic gap, it is vital to develop methods and policies effective for smokers with a lower socioeconomic status.

Having to pay for smoking cessation treatment like bupropion, varenicline or nicotine replacement therapy-from here on referred to as'cessation medication'- can be a barrier for seeking treatment, particularly for people on a low income. ${ }^{14}$ More smokers use cessation medication when it is cost-free, ${ }^{15}$ and this can substantially increase the number of longterm quitters. ${ }^{16}$ In a study based on data from the International Tobacco Control (ITC) Europe surveys, respondents with lower education and income mentioned the availability of free/lower cost medication more often as a trigger for thinking about quitting smoking than other education and income groups. ${ }^{17}$ Still, mentioning free/lower cost medication as a trigger for thinking about quitting does not necessarily lead to more use of medication, nor to more quit attempts and a greater likelihood of quit success. Data from the ITC Four Country surveys ${ }^{18}$ suggested that mentioning free/lower cost medication as a trigger to think about quitting was positively associated with making a quit attempt. Yet, it was not examined whether this association was dependent on respondents' income or education. Furthermore, it was not assessed whether free/lower cost medication as a trigger was associated with actual use of cessation medication and quit success.

In the current study, the ITC data from the UK and the Netherlands is used to investigate SES differences in mentioning free/lower cost medication as a trigger to think about quitting smoking and quitting behavior. The cultural and economic similarities of the UK and the Netherlands make it interesting to compare these two countries. In both countries, smokers have the opportunity to receive free/lower cost smoking cessation treatment. In the UK, the National Health Service provides smokers with free or subsidized cessation medication (dependent on smokers' income) by trained practitioners in routine practice and via the stop-smoking services; behavioral support can also be obtained free via the stop-smoking services for all. ${ }^{19,20}$ Stop smoking services vary across the UK, but the standard model of treatment is an assessment before quitting which takes place about a week later, and then weekly sessions until four weeks after the quit date. ${ }^{21}$ In the Netherlands, pharmacotherapy can be reimbursed once every year by the Dutch health insurance system only in combination with behavioral therapy, irrespective of treatment completion or outcome. There are possibilities for receiving behavioral therapy, such as the general practitioner, outpatient services, or independent healthcare providers registered 
in the Dutch quality register for scientifically proven effective smoking cessation treatment. Still, reimbursement applies only after patients have spent a mandatory deductible for general healthcare costs (at least 350 euro in 2013).

The aim of this study is to investigate whether in the UK and the Netherlands free/lower cost cessation medication mentioned as a trigger for thinking about quitting is related to use of medication, quit attempts and quit success and whether these associations are modified by education and income. It is hypothesized that smokers who mentioned free or lower cost medication as a trigger were more likely to make a quit attempt, to use cessation medication and had higher quit rates. Moreover, it is expected that this association is stronger for smokers with lower income and education than smokers with higher income and education, as the cost of cessation treatment has been mentioned as a barrier to quitting smoking by smokers with lower income and education. ${ }^{22,23}$

\section{METHODS}

Data were used from the 2013 and 2014 surveys of the ITC Netherlands and UK cohorts. The ITC Project is a prospective cohort study of a representative sample of smokers and ex-smokers in each country. ${ }^{24}$ Survey data were collected by web (59\%) and telephone $(41 \%)$ for the UK and by web only for the Netherlands. Surveys were collected from February to September 2013 and from August to December 2014 in the UK, and from May to June 2013 and from May to June 2014 in the Netherlands. All respondents were current smokers at time of enrolment. More details on the methods of the ITC data collection can be found in previous publications. ${ }^{24,25}$ Drop out between the two survey waves was $18 \%$ for the Netherlands and $30 \%$ for the UK. In the current study, participants were selected of 18 years and older who participated in both waves and who smoked at least monthly in the 2013 survey. This formed a sample of $n=1164$ smokers from the Netherlands, and $n=768$ from the UK.

\section{Free/lower cost medication as a trigger for thinking about quitting}

The main independent variable of this study was whether respondents mentioned free/ lower cost smoking cessation medication as a trigger for thinking about quitting. This is referred to as 'free/lower cost medication as a trigger' in the remainder of this paper. It was measured in the 2013 surveys with the following question:'In the past 6 months, has free, or lower cost, stop-smoking medication led you to think about quitting? ${ }^{17}$.

The question was part of a list with four policy triggers, of which each could be selected independently from the others by the respondent. The other triggers (which were not the focus in this paper) were: the price of cigarettes, smoking restrictions in public places and warning labels on cigarette packages. Response options were: 'not at all', 'somewhat' and 'very much'. For the analyses, the response options were dichotomized: 'not at all' was classified as no trigger for thinking about quitting; 'somewhat' and 'very much' was classified as a positive trigger for thinking about quitting smoking. 


\section{Use of smoking cessation medication}

Participants in both countries were asked in the 2014 surveys whether they had previously used smoking cessation medications. In the Netherlands, respondents were asked about medication use in the last year while in the UK, medication use during the last quit attempt was assessed. Therefore, these survey items were not entirely comparable.

\section{Quit attempts and quit success}

To measure quit attempts, participants were asked the following question in the 2014 survey: 'Have you made any attempts (successful or not) to stop smoking in the last year? Participants who responded affirmatively on this question were defined as having made a quit attempt. Successful quitters were defined as current smokers in 2013 who reported having made a quit attempt and not smoking at all or less than once a month in 2014 . ${ }^{26}$

\section{Income and education}

Respondents from the Netherlands were asked about their monthly gross household income while UK respondents reported their annual gross household income. Response categories also differed between the two countries. The income variables were recoded into three categories based on tertiles in each country: defined as 'low' if income $<€ 2000$ (NL) or $<£ 15000$ (UK), 'moderate' if between $€ 2000$ and $€ 3000$ (NL) or between $£ 15000$ and $£ 30000$ (UK) and 'high' if $>€ 3000$ (NL) or $>£ 30000$ (UK). Respondents had the option not to disclose their income. These responses were treated as missing values. The level of education for both countries was recoded into three groups: 'low' for none completed, elementary school and lower secondary education; 'moderate' for secondary vocational education and middle secondary education; and 'high' for upper secondary education, university and post-graduate.

\section{Covariates}

Several covariates were used in the analyses, including gender and age (divided into four categories: 18-24 years, 25-39 years, 40-54 years, and 55 years and older). Nicotine dependence was measured by the Heaviness of Smoking Index (HSI). ${ }^{27,28}$ The HSI is a score ranging from 0-6 (low to high nicotine dependence) and is calculated based on both the number of cigarettes smoked per day and the time before smoking the first cigarette after waking up.

\section{Analyses}

IBM SPSS version $21.0{ }^{29}$ was used to analyze the data. Cross-sectional and longitudinal weights were calculated using age and gender for each country separately. ${ }^{24}$ Three multivariate logistic regression analyses were used to analyze the data. The associations were assessed between mentioning free/lower cost medication as a trigger to quit and (1) use of smoking cessation medication, (2) making a quit attempt and (3) smoking cessation one year later. Covariates in all analyses were gender, age, and HSI. To account for possible "time-in-sample" effects where answers from respondents who have participated in multiple survey waves vary from newly recruited respondents, ${ }^{30}$ analyses were adjusted for the respondents' number of times they participated in the survey. 31 Two-way interactions between education and trigger and between income and trigger were included in the analyses. Due to between-country differences in data collection 
and survey items, analyses were conducted separately for the Netherlands and the UK. As secondary analyses, the other three policy triggers (the price of cigarettes, smoking restrictions in public places and warning labels on cigarette packages) were added as independent variables in separate multivariate logistic regression analyses.

\section{RESULTS}

\section{Demographics}

The socio-demographic characteristics of the respondents are presented in Table 1. The UK research sample had a higher percentage $(53 \%)$ of high educated smokers than the Netherlands $(25 \%)(p<0.001)$. In the Netherlands, $30 \%$ of respondents did not report their household income, compared to $8 \%$ in the UK. Appendix 1 shows medication use, quit attempts and quit success for respondents who did and who did not disclose their income. Smokers in both countries had a comparable level of nicotine dependence $(p=$ 0.448), with the largest group of respondents reporting an HSI between 2 and 4. Loss to follow up was higher among younger participants in both countries and higher among lighter smokers in the UK (Appendix 2).

Table 1. Characteristics of participants in the first wave in the UK and the Netherlands (2013). Weighted data.

\begin{tabular}{lccc}
\hline & $\begin{array}{c}\text { United Kingdom } \\
(\mathbf{n}=\mathbf{7 6 8})\end{array}$ & $\begin{array}{c}\text { The Netherlands } \\
(\mathbf{n}=\mathbf{1 1 6 4 )}\end{array}$ & $\begin{array}{c}\text { Between country } \\
\text { differences }\end{array}$ \\
\hline Gender & & & \\
Female (\%) & 48.6 & 49.3 & $\begin{array}{c}\chi^{2}(1)=0.08 \\
\mathrm{p}=(0.777)\end{array}$ \\
& & & \\
Age & 11.9 & 12.8 & $\chi^{2}(3)=4.90$ \\
18-24 years (\%) & 27.2 & 23.1 & $(\mathrm{p}=0.179)$ \\
25-39 years (\%) & 33.4 & 33.4 & \\
40-54 years (\%) & 27.6 & 30.6 & \\
55 years and older (\%) & & & \\
Heaviness of Smoking Index & 27.5 & 29.6 & $(\mathrm{p}=0.448)$ \\
0-1 (\%) & 65.8 & 63.0 & \\
2-4 (\%) & 6.7 & 7.5 & \\
5-6 (\%) & & & $\chi^{2}(2)=144.40$ \\
Income level & 28.5 & 22.0 & \\
Low (\%) & 31.7 & 20.1 & \\
Moderate (\%) & 32.0 & 27.8 & \\
High (\%) & 7.7 & 30.1 & \\
Not reported (\%) & & & $\chi^{2}(2)=146.59$ \\
Educational level & 17.4 & 29.2 & $(\mathrm{p}<0.001)$ \\
Low (\%) & 30.1 & 45.4 & \\
Moderate (\%) & 52.5 & 25.4 & \\
High (\%) & & & \\
\hline
\end{tabular}




\section{Free/lower cost medication as a trigger for thinking about quitting}

In 2013, free/lower cost medication was mentioned as a trigger for thinking about quitting by $24.9 \%$ of the respondents in the Netherlands and $37.0 \%$ of the respondents in the UK (results not shown in table). When comparing income groups, free/lower cost medication as a trigger was mentioned in the Netherlands by $30.7 \%$ of low income smokers compared to $20.9 \%$ of moderate income smokers and $21.1 \%$ of high income smokers $\left(\chi^{2}(2)=9.45\right.$, $\mathrm{p}=0.009)$. In the UK, free/lower cost medication as a trigger was mentioned by $40.2 \%$, $33.6 \%$ and $39.2 \%$ of low, moderate and high income groups respectively $\left(\chi^{2}(2)=2.47, p\right.$ $=0.290$ ). When education groups were compared, in the Netherlands $28.8 \%$ of smokers with low education, $25.7 \%$ of smokers with moderate education and $21.2 \%$ of smokers with high education mentioned free/lower cost medication as a trigger $\left(\chi^{2}(2)=4.98, p=\right.$ 0.083). In the UK, $38.8 \%$ of smokers with low education, $41.1 \%$ of smokers with moderate education, and $34.1 \%$ smokers with high education mentioned free/lower cost medication as a trigger $\left(\chi^{2}(2)=3.17, p=0.205\right)$.

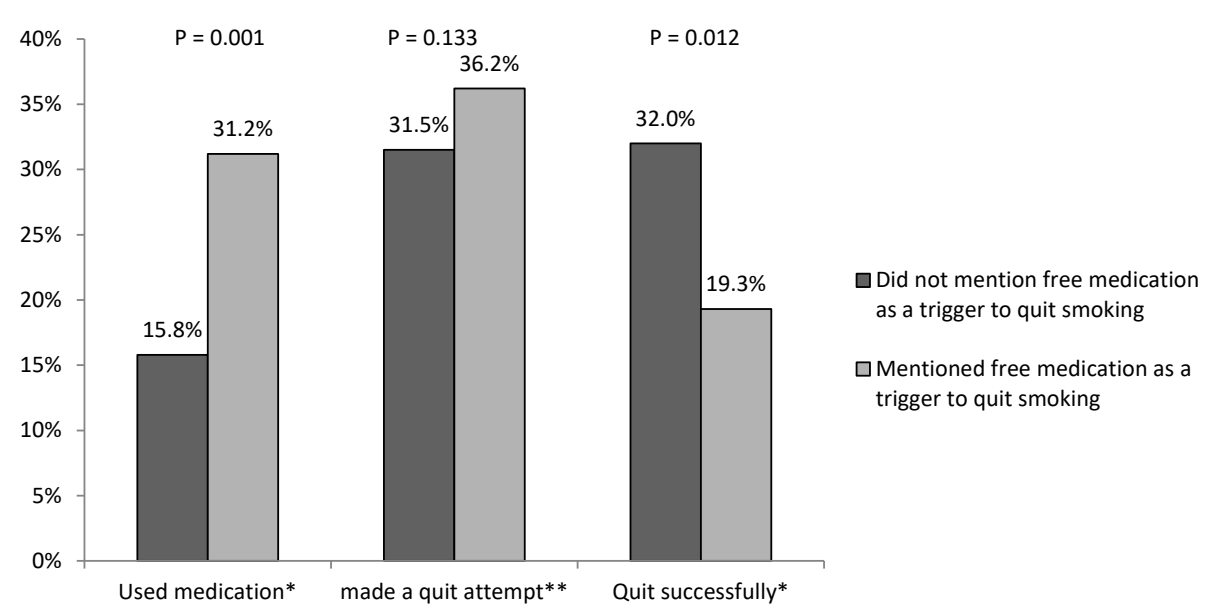

Figure 1. Medication use, quit attempts and successful quitters among smokers who mentioned and smokers who did not mention free/lower cost medication as a trigger to think about quitting smoking in the Netherlands. *The denominator is all participants who made a quit attempt in the last year. ${ }^{* *}$ The denominator is all smokers. 


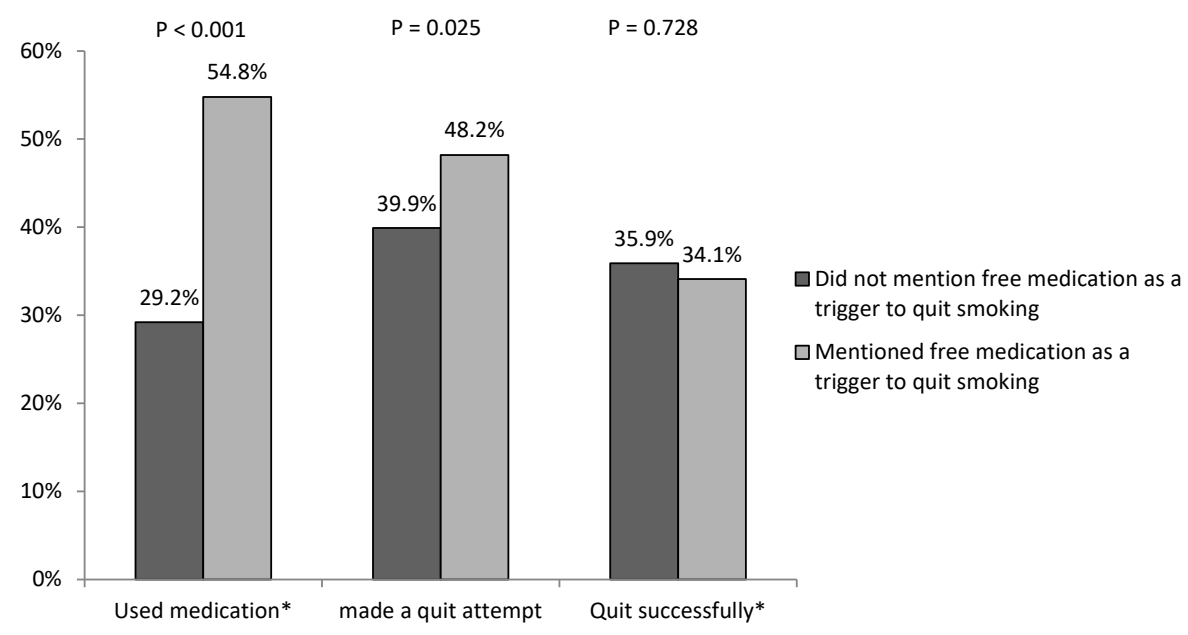

Figure 2. Medication use, quit attempts and successful quitters among smokers who mentioned and smokers who did not mention free/lower cost medication as a trigger to think about quitting smoking in the UK. *The denominator is all participants who made a quit attempt in the last year.**The denominator is all smokers.

\section{Medication use}

In the Netherlands, $31.2 \%$ of smokers who made a quit attempt in the last year and mentioned free/lower cost medication as a trigger, used smoking cessation medication in the last year compared to $15.8 \%$ who did not report this trigger $(p=0.001$, Figure 1 and Table 2). In the UK, the medication use in the group of respondents that mentioned free/ lower cost medication as a trigger was $54.8 \%$ compared to $29.2 \%$ in the group that did not report the trigger ( $p<0.001$, Figure 2$)$. Free/lower cost medication as a trigger was also positively associated with medication use in a multivariate analyses both in the UK (OR = 4.19, $\mathrm{p}<0.001)$ and in the Netherlands $(\mathrm{OR}=2.14, \mathrm{p}=0.033)$ (Table 3). The multivariate analyses showed no significant interactions between free/lower cost medication as a trigger, medication use, and education and income groups. With the other policy triggers (smoking restrictions in public places, the price of cigarettes and warning labels on cigarette packages) added to the model, free/lower cost medication as a trigger was no longer significantly associated with medication use in the Netherlands $(O R=1.83, p=$ $0.113)$, but remained significant in the UK $(O R=4.26, p<0.001)$ (results not shown in table). 
Table 2. Medication use, quit attempts and quit success within the entire sample and within respondents who mentioned free/lower cost medication as a trigger (weighted data).

\begin{tabular}{lcccccc}
\hline & & Entire sample & \multicolumn{4}{c}{$\begin{array}{c}\text { Mentioned free/lower cost medication } \\
\text { as a trigger }\end{array}$} \\
\hline & UK & NL & p-value & UK & NL & p-value \\
\hline Medication use (\%) & 39.8 & 20.1 & $<0.001$ & 54.8 & 31.2 & $<0.001$ \\
Quit attempts (\%) & 43.0 & 32.7 & $<0.001$ & 48.2 & 36.2 & 0.003 \\
Quit success (\%) & 35.2 & 28.5 & 0.064 & 34.1 & 19.3 & 0.014 \\
\hline
\end{tabular}

\section{Quit attempts}

A bivariate analysis showed that $36.2 \%$ of respondents in the Netherlands who mentioned free/lower cost medication as a trigger made at least one quit attempt between 2013 and 2014 compared to $31.5 \%$ who did not report this trigger $(p=0.133$, see Figure 1$)$. In the UK, these numbers were $48.2 \%$ versus $39.9 \%$ ( $p=0.025$, see Figure 2 ). The multivariate analyses showed that in the UK, respondents who mentioned free/lower cost medication as a trigger had a higher rate of quit attempts within the next year than those who did not report this trigger ( $O R=1.45, p=0.030)$, but this association was not found in the Netherlands $(O R=1.10, p=0.587$, see Table 3 ). There were no significant interactions between free/lower cost medication as a trigger and education and income levels for quit attempts in the multivariate analyses. When other policy triggers were added to the analysis as independent variables, free/lower cost medication as a trigger was no longer significantly associated with quit attempts in the UK $(O R=1.14, p=0.489)$, and remained non-significant in the Netherlands.

\section{Quit success}

Within the group of smokers who made a quit attempt, in the Netherlands $19.3 \%$ of respondents who mentioned medication as a trigger quit smoking successfully compared to $32.0 \%$ who did not report this trigger ( $p=0.012$, Figure 1$)$. In the UK, this was $34.1 \%$ versus $35.9 \%$ ( $p=0.728$, Figure 2). The multivariate analyses showed no significant association between free/lower cost medication as a trigger for thinking about quitting smoking and quit success (Table 3). The analyses showed no significant interactions between free/ lower cost medication as a trigger and quit success for different income and education levels. With the other three policy triggers added to the model, the association between free/lower cost medication as a trigger and quit success remained not significant. 


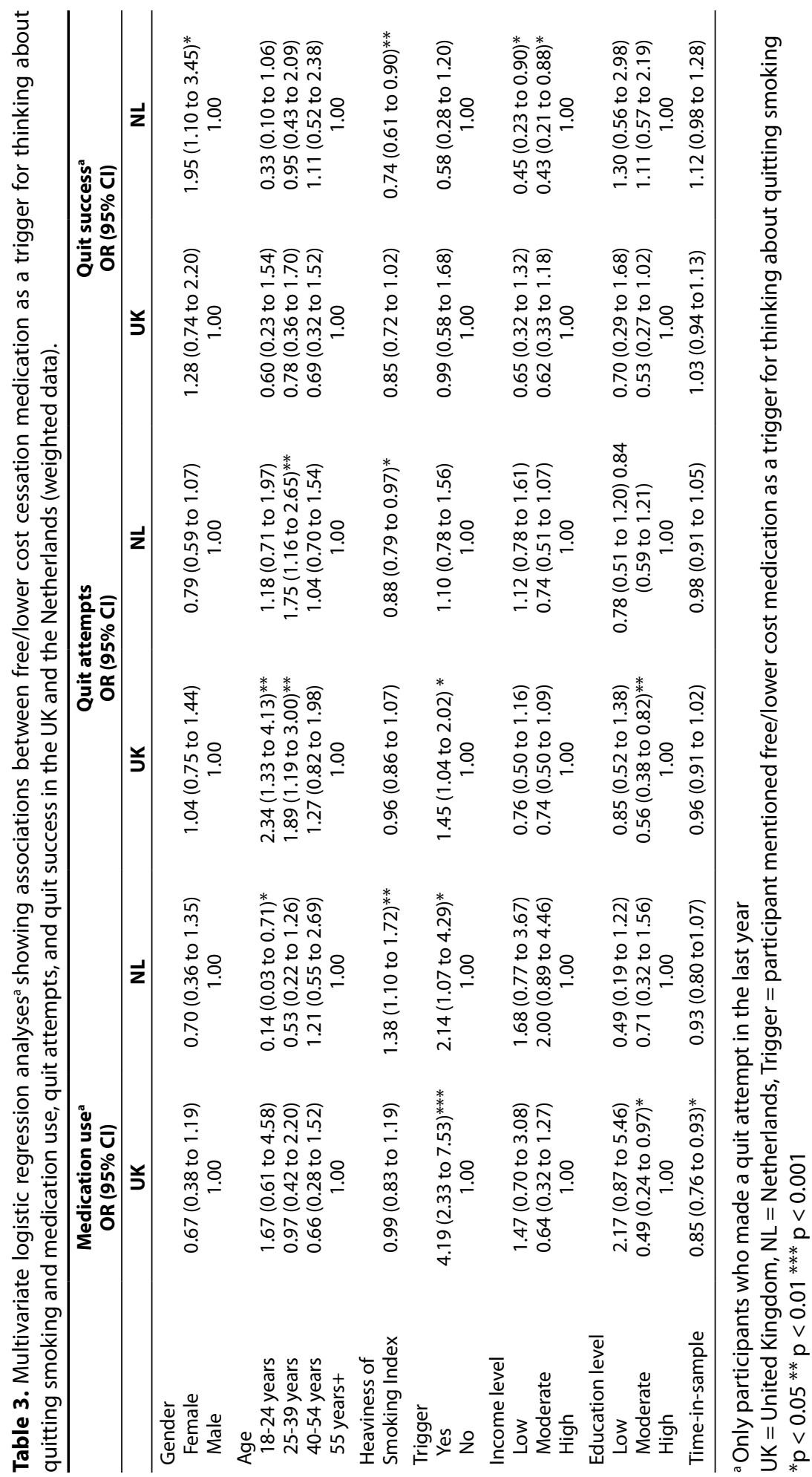




\section{DISCUSSION}

The aim of this study was to investigate whether mentioning free/lower cost medication as a trigger to think about quitting smoking was related to the use of cessation medication, quit attempts and quit success in the UK and the Netherlands, and whether these associations were modified by education and income. The results showed a positive association between mentioning free/lower cost medication as a trigger and the use of smoking cessation medication in both the UK and the Netherlands. This finding is in line with previous research, which showed that financial coverage leads to an increased utilization of smoking cessation medication. ${ }^{15}$

Smokers who mentioned free/lower cost medication as a trigger for thinking about quitting smoking were more likely to have made a quit attempt a year later in the UK, but not in the Netherlands. The association between free/lower cost medication as a trigger to quit smoking and quit attempts has also been demonstrated in Canada, the USA and Australia. ${ }^{18}$ The finding that there was no significant association between free/ lower cost medication as a trigger and quit attempts in the Netherlands may result from the difference in availability of free/lower cost medication compared to the UK. The UK's stop smoking services and health professionals provide subsidized medications for any smokers who want to quit, while in the Netherlands health insurance reimbursement for smoking cessation medication is only available after a mandatory deductible amount is first spent. Not being able to receive free medication could demotivate smokers to follow through with their intended quit attempt. ${ }^{22,32}$

Smokers who mentioned free/lower cost medication as a trigger were not more likely to quit successfully which emphasizes that it is a large step from contemplating quitting to actually achieving this goal. Firstly, there may be barriers preventing smokers to start and sustain a quit attempt with medication, such as availability of free medication, self-efficacy and motivation. ${ }^{32-35}$ Likewise, many factors can influence whether a quit attempt leads to successful quitting. ${ }^{36}$ The current study did not investigate which hindering or promoting factors influence the relation between free/lower cost medication as a trigger and quit success, but further research focusing on this question could give important insights in how to achieve quit success in smokers who are triggered to think about quitting by free medication.

When the other policy triggers (smoking restrictions in public places, the price of cigarettes and warning labels on cigarette packages) were added to the analyses, the associations between free/lower cost medication as a trigger and the use of medication in the Netherlands, and quit attempts in the UK were no longer significant. However, the association between free/lower cost medication as a trigger and the use of smoking cessation medication in the UK remained significant after correcting for the other policy triggers, which makes this the most robust finding of this study.

Another aim of this study was to investigate whether the income and education level of smokers influenced the association between mentioning free/lower cost medication as a trigger to quit smoking and use of cessation medications, quit attempts, and quit success. 
Contrary to our hypothesis, the results showed that this association was not influenced by education or income level. This may mean that smokers with a lower SES as well as smokers with a higher SES who are triggered to think about quitting smoking by free cessation medication are equally likely to use medication, to make a quit attempt, and potentially be successful in this attempt.

\section{Limitations}

This study has some limitations that should be taken into consideration. The validity of the survey question about whether respondents considered free/lower cost medication as a trigger to quit smoking was assumed but not investigated in this study. Loss to follow-up was higher among younger respondents in both countries, which could have influenced the results since research has shown that young adults were more likely to try quitting smoking, were more successful in quitting and were less likely to use cessation medication than older adults. ${ }^{37,38}$ The UK and Netherlands data were not completely comparable since there were significant differences in income and education levels and differences in measuring the use of smoking cessation medication. In the UK, medication use was measured for the last quit attempt while in the Netherlands it was measured for the last year. Therefore, the use of cessation medication may have predated the (by free medication triggered) thought about quitting smoking. In the UK, the lower education group was underrepresented. In the Netherlands, a large portion of the respondents did not disclose their income, which could have influenced the results if income was not equally distributed among these non-responders. Additionally, in the Netherlands, data were collected by web questionnaires only and in the UK also by telephone. This difference in data collection could have affected survey outcomes but was expected to be of minor influence on the results of this study. ${ }^{39}$

\section{Practical implications}

This study found that smokers who considered free/lower cost medication as a trigger to quit smoking were more likely to actually use cessation medication, and that smokers from the UK were more likely to have attempted to quit smoking. To promote smoking cessation, it may therefore be beneficial to raise awareness of the availability of free cessation medication, for example through mass media campaigns or healthcare providers.

The finding that the association between mentioning free/lower cost medication as a trigger for thinking about quitting smoking and actual quit attempts was not influenced by education or income level suggests that free medication can motivate a large part of the smoking population to quit, and that this effect is not restricted to particular socioeconomic groups. Yet, since free medication is mentioned more often as a trigger to quit smoking by lower income smokers in the Netherlands in the current study and in previous research also in the UK, Ireland and Germany, ${ }^{17}$ free medication may be an important strategy to decrease the socioeconomic gap in smoking. 


\section{Conclusion}

Considering free/lower cost smoking cessation medication as a trigger for thinking about quitting smoking could, irrespective of smokers' income or education, be positively associated with quit attempts and may promote the use of cessation medication during this attempt. Therefore, making cessation medication freely accessible to smokers may be an important strategy to decrease smoking in the population. 


\section{REFERENCES}

1. Reid JL, Hammond D, Boudreau C, Fong GT, Siahpush M. Socioeconomic disparities in quit intentions, quit attempts, and smoking abstinence among smokers in four western countries: findings from the International Tobacco Control Four Country Survey. Nicotine \& tobacco research : official journal of the Society for Research on Nicotine and Tobacco. 2010;12 Suppl:S20-33.

2. Hiscock R, Bauld L, Amos A, Platt S. Smoking and socioeconomic status in England: the rise of the never smoker and the disadvantaged smoker. J Public Health (Oxf). 2012;34(3):390-396.

3. Nagelhout GE, de Korte-de Boer $D$, Kunst $A E$, et al. Trends in socioeconomic inequalities in smoking prevalence, consumption, initiation, and cessation between 2001 and 2008 in the Netherlands. Findings from a national population survey. BMC Public Health. 2012;12(1):1-9.

4. Huisman M, Kunst AE, Mackenbach JP. Inequalities in the prevalence of smoking in the European Union: comparing education and income. Prev Med. 2005;40(6):756-764.

5. Schaap MM, Kunst AE. Monitoring of socio-economic inequalities in smoking: Learning from the experiences of recent scientific studies. Public Health. 2009;123(2):103-109.

6. Siahpush M, English D, Powles J. The contribution of smoking to socioeconomic differentials in mortality: results from the Melbourne Collaborative Cohort Study, Australia. J Epidemiol Community Health. 2006;60(12):1077-1079.

7. Jha P, Peto R, Zatonski W, Boreham J, Jarvis MJ, Lopez AD. Social inequalities in male mortality, and in male mortality from smoking: indirect estimation from national death rates in England and Wales, Poland, and North America. The Lancet. 2006;368(9533):367-370.

8. Giskes K, Kunst AE, Benach J, et al. Trends in smoking behaviour between 1985 and 2000 in nine European countries by education. J Epidemiol Community Health. 2005;59(5):395-401.

9. Gielkens-Sijstermans CM, Mommers MA, Hoogenveen RT, et al. Reduction of smoking in Dutch adolescents over the past decade and its health gains: a repeated cross-sectional study. Eur $J$ Public Health. 2010;20(2):146-150.

10. Harper S, Lynch J. Trends in socioeconomic inequalities in adult health behaviors among US states, 1990-2004. Public Health Rep. 2007;122(2):177-189.

11. Federico B, Costa G, Kunst AE. Educational inequalities in initiation, cessation, and prevalence of smoking among 3 Italian birth cohorts. Am J Public Health. 2007;97(5):838-845.

12. Office for National Statistics. Dataset: Smoking habits in the UK and its constituent countries. In:2016.

13. Verdurmen J, Monshouwer K, Van Laar M, Van Bon-Martens M. Factsheet Continu Onderzoek Rookgewoonten 2013. Utrecht: Trimbos-instituut;2014.

14. Roddy E, Antoniak M, Britton J, Molyneux A, Lewis S. Barriers and motivators to gaining access to smoking cessation services amongst deprived smokers - a qualitative study. BMC Health Serv Res. 2006;6(1):147.

15. Van den Brand FA, Nagelhout $G E$, Reda AA, et al. Healthcare financing systems for increasing the use of tobacco dependence treatment. Cochrane Database Syst Rev. 2017(9).

16. Kaper J, Wagena EJ, Willemsen MC, Van Schayck CP. Reimbursement for smoking cessation treatment may double the abstinence rate: results of a randomized trial. Addiction. 2005;100(7):1012-1020.

17. Hummel K, Nagelhout GE, Willemsen MC, et al. Trends and socioeconomic differences in policy triggers for thinking about quitting smoking: Findings from the International Tobacco Control (ITC) Europe Surveys. Drug Alcohol Depend. 2015;155:154-162. 
18. Kasza KA, Hyland AJ, Borland R, et al. Cross-country comparison of smokers' reasons for thinking about quitting over time: findings from the International Tobacco Control Four Country Survey (ITC-4C), 2002-2015. Tob Control. 2016.

19. Bauld L, Bell K, McCullough L, Richardson L, Greaves L. The effectiveness of NHS smoking cessation services: a systematic review. Journal of Public Health. 2010;32(1):71-82.

20. NHS. National Health Service. https://www.nhs.uk/smokefree. Accessed 31-03-2017.

21. NCSCT. Standard treatment programme. A guide to providing behavioural support for smoking cessation National Centre for Smoking Cessation and Training. http://www.ncsct.co.uk/usr/ pub/standard_treatment_programme.pdf. Published 2014. Accessed 22-01-2018, 2018.

22. Rosenthal L, Carroll-Scott A, Earnshaw VA, et al. Targeting cessation: Understanding barriers and motivations to quitting among urban adult daily tobacco smokers. Addict Behav. 2013;38(3):1639-1642.

23. Bryant J, Bonevski B, Paul C, O'Brien J, Oakes W. Developing cessation interventions for the social and community service setting: A qualitative study of barriers to quitting among disadvantaged Australian smokers. BMC Public Health. 2011;11(1):493.

24. Thompson ME, Fong GT, Hammond D, et al. Methods of the International Tobacco Control (ITC) Four Country Survey. Tob Control. 2006;15(suppl 3):iii12-iii18.

25. Zethof $D$, Nagelhout GE, de Rooij M, et al. Attrition analysed in five waves of a longitudinal yearly survey of smokers: findings from the ITC Netherlands survey. Eur J Public Health. 2016;26(4):693699.

26. Hyland A, Borland R, Li Q, et al. Individual-level predictors of cessation behaviours among participants in the International Tobacco Control (ITC) Four Country Survey. Tob Control. 2006;15(suppl 3):iii83-iii94.

27. Heatherton TF, Kozlowski LT, Frecker RC, Fagerstrom K-O. The Fagerström Test for Nicotine Dependence: a revision of the Fagerstrom Tolerance Questionnaire. Br JAddict. 1991;86(9):11191127.

28. Heatherton TF, Kozlowski LT, Frecker RC, Rickert W, Robinson J. Measuring the Heaviness of Smoking: using self-reported time to the first cigarette of the day and number of cigarettes smoked per day. Br J Addict. 1989;84(7):791-799.

29. IBM Corp. IBM SPSS Statistics for Windows, Version 21.0 [computer program]. Version Version 21.0. Armonk, NY: IBM Corp.; 2012.

30. Thompson ME. Using Longitudinal Complex Survey Data. Annual Review of Statistics and Its Application. 2015;2(1):305-320.

31. Driezen $P$, Thompson M. Comparing policy measures across multiple ITC countries: Adjusting for time-in-sample. University of Waterloo. 2011.

32. Smith AL, Carter SM, Chapman S, Dunlop SM, Freeman B. Why do smokers try to quit without medication or counselling? A qualitative study with ex-smokers. BMJ Open. 2015;5(4):e007301.

33. Jardin $\mathrm{BF}$, Cropsey $\mathrm{KL}$, Wahlquist $\mathrm{AE}$, et al. Evaluating the effect of access to free medication to quit smoking: a clinical trial testing the role of motivation. nicotine \& tobacco research. 2014:ntu025.

34. Twyman L, Bonevski B, Paul C, Bryant J. Perceived barriers to smoking cessation in selected vulnerable groups: a systematic review of the qualitative and quantitative literature. BMJ Open. 2014;4(12):e006414.

35. Gwaltney CJ, Metrik J, Kahler CW, Shiffman S. Self-efficacy and smoking cessation: a metaanalysis. Psychol Addict Behav. 2009;23(1):56-66. 
36. Vangeli E, Stapleton J, Smit ES, Borland R, West R. Predictors of attempts to stop smoking and their success in adult general population samples: a systematic review. Addiction. 2011;106(12):21102121.

37. Messer K, Trinidad DR, Al-Delaimy WK, Pierce JP. Smoking Cessation Rates in the United States: A Comparison of Young Adult and Older Smokers. Am J Public Health. 2008;98(2):317-322.

38. Curry SJ, Sporer AK, Pugach O, Campbell RT, Emery S. Use of Tobacco Cessation Treatments Among Young Adult Smokers: 2005 National Health Interview Survey. Am J Public Health. 2007;97(8):1464-1469.

39. Nagelhout GE, Willemsen MC, Thompson ME, Fong GT, van den Putte $B$, de Vries $H$. Is web interviewing a good alternative to telephone interviewing? Findings from the International Tobacco Control (ITC) Netherlands Survey. BMC Public Health. 2010;10(1):351. 


\section{APPENDIX 1}

Proportion of smokers from the Netherlands and the UK who made a quit attempt, used medications and who quit successfully for respondents with different income levels and respondents who did not report their income (weighted data).

\begin{tabular}{|c|c|c|c|c|c|}
\hline & \multicolumn{4}{|c|}{ Income level } & \multirow[b]{2}{*}{ p-value } \\
\hline & Low & Moderate & High & Not reported* & \\
\hline \multicolumn{6}{|c|}{ Quit attempt (\%) } \\
\hline UK & 37.4 & 38.2 & 50.0 & 50.0 & 0.012 \\
\hline NL & 36.0 & 29.6 & 36.2 & 27.6 & 0.037 \\
\hline \multicolumn{6}{|c|}{ Used medication (\%) } \\
\hline UK & 50.6 & 31.5 & 39.5 & 37.9 & 0.085 \\
\hline NL & 26.3 & 23.3 & 15.4 & 18.6 & 0.214 \\
\hline \multicolumn{6}{|c|}{ Quit success (\%) } \\
\hline UK & 31.2 & 27.2 & 41.9 & 41.4 & 0.107 \\
\hline $\mathrm{NL}$ & 21.3 & 21.9 & 41.5 & 23.5 & 0.002 \\
\hline
\end{tabular}

* In the UK 7.7\% did not report their income, in the Netherlands $30.1 \%$ 


\section{APPENDIX 2}

Baseline characteristics of respondents included in the analysis and respondents lost to follow-up for the Netherlands and the UK.

\begin{tabular}{|c|c|c|c|c|c|c|}
\hline & \multicolumn{2}{|c|}{ United Kingdom } & \multicolumn{4}{|c|}{ The Netherlands } \\
\hline & $\begin{array}{c}\text { Included in } \\
\text { analysis } \\
(n=768)\end{array}$ & $\begin{array}{c}\text { Lost to } \\
\text { follow-up } \\
(n=335)\end{array}$ & p-value & $\begin{array}{l}\text { Included in } \\
\text { analysis } \\
(n=1164)\end{array}$ & $\begin{array}{l}\text { Lost to } \\
\text { follow-up } \\
(n=256)\end{array}$ & p-value \\
\hline \multicolumn{7}{|l|}{ Gender } \\
\hline Female (\%) & 52.6 & 47.2 & 0.102 & 49.0 & 45.3 & 0.301 \\
\hline \multicolumn{7}{|l|}{ Age } \\
\hline $18-24$ years $(\%)$ & 3.8 & 9.6 & $<0.001$ & 17.9 & 23.4 & $<0.001$ \\
\hline $25-39$ years $(\%)$ & 21.9 & 30.1 & & 27.8 & 36.3 & \\
\hline $40-54$ years $(\%)$ & 42.2 & 36.1 & & 30.7 & 28.9 & \\
\hline $55+$ years $(\%)$ & 32.2 & 24.2 & & 23.6 & 11.3 & \\
\hline \multicolumn{7}{|c|}{ Heaviness of Smoking Index } \\
\hline $0-1(\%)$ & 27.0 & 36.3 & 0.011 & 31.1 & 27.2 & 0.075 \\
\hline $2-4(\%)$ & 65.4 & 57.4 & & 62.2 & 69.0 & \\
\hline $5-6(\%)$ & 7.5 & 6.3 & & 6.7 & 3.8 & \\
\hline \multicolumn{7}{|l|}{ Income level } \\
\hline Low (\%) & 31.0 & 32.2 & 0.863 & 21.4 & 19.1 & 0.646 \\
\hline Moderate (\%) & 31.1 & 28.7 & & 18.9 & 21.9 & \\
\hline High (\%) & 29.9 & 31.3 & & 29.2 & 27.7 & \\
\hline Not reported (\%) & 7.9 & 7.8 & & 30.5 & 31.2 & \\
\hline \multicolumn{7}{|l|}{ Educational level } \\
\hline Low (\%) & 21.4 & 16.6 & 0.054 & 26.3 & 26.0 & 0.770 \\
\hline Moderate (\%) & 27.2 & 24.4 & & 46.9 & 49.2 & \\
\hline High (\%) & 51.5 & 59.0 & & 26.7 & 24.8 & \\
\hline
\end{tabular}


CHAPTER 4 


\section{The effect of financial incentives on top of behavioral support on quit rates in tobacco smoking employees: study protocol of a cluster-randomized trial}

Van den Brand FA, Nagelhout GE, Winkens B, et al. The effect of financial incentives on top of behavioral support on quit rates in tobacco smoking employees: study protocol of a cluster-randomized trial 


\section{ABSTRACT}

Background: Stimulating successful tobacco cessation among employees has multiple benefits. Employees who quit tobacco are healthier, more productive, less absent from work, and longer employable than employees who continue to use tobacco. Despite the evidence for these benefits of tobacco cessation, a successful method to stimulate employees to quit tobacco is lacking. The aim of this study is to evaluate whether adding a financial incentive to behavioral support (compared with no additional incentive) is effective and cost-effective in increasing abstinence rates in tobacco smoking employees participating in a smoking cessation group training.

Methods/design: In this cluster-randomized trial employees in the intervention and control group both participate in a smoking cessation group training consisting of seven weekly counseling sessions of ninety minutes each. In addition to the training, employees in the intervention group receive a voucher as an incentive for being abstinent from smoking at the end of the training (€50), after three months ( $€ 50)$, after six months $(€ 50)$, and after one year $(€ 200)$. The control group does not receive any incentive. The primary outcome is carbon monoxide validated 12-month continuous abstinence from smoking (Russel's standard). Additionally, an economic evaluation is performed from a societal and an employer perspective.

Discussion: The present paper describes the methods and design of this clusterrandomized trial in detail. We hypothesize that the financial incentive for abstinence in the form of vouchers increases abstinence rates over and above the group training. The results of this study can provide important recommendations for enhancement of employee tobacco cessation. 


\section{INTRODUCTION}

Tobacco use is a major health threat and responsible for deaths due to cancer, chronic obstructive pulmonary disease (COPD), coronary heart disease, stroke and heart failure. ${ }^{1}$ The life expectancy for smokers is at least 10 years shorter than for nonsmokers. ${ }^{1,2}$ Not only is smoking a serious health risk, it is also responsible for an estimated $8.7 \%$ of annual healthcare costs in the US. ${ }^{3}$ Employees who smoke also represent a significant cost to their employers. ${ }^{4}$ These costs can be distinguished as the cost due to sickness absenteeism and the cost due to smoking breaks. Smokers are about 1.5 more days per year absent from work than non-smokers. ${ }^{5,6}$ Smoking is associated with absenteeism, ${ }^{5,7}$ and with reduced performance. 8,9 The amount of time that employees spend smoking each workday depends on several factors, such as the number of smoking breaks and whether the employee has to go outside to smoke. An employee who works fulltime and takes four smoking breaks of ten minutes per day spends about 150 (work) hours per year on smoking. It was estimated that in the US, employees who smoke cost their employer an excess of $\$ 5816$ per smoker per year. ${ }^{4}$ On the other hand, when employees quit smoking, their absenteeism declines within several years. ${ }^{5}$ In addition, the productivity of former smokers is higher than that of current smokers. ${ }^{5}$ It is therefore profitable for employers to invest in smoking cessation both from a company perspective as well as from a societal viewpoint.

An approach in stimulating employees to stop smoking is to use incentives for quit success. The rationale behind incentives for healthy behavior is twofold. Firstly, people value present benefits and costs more than future ones. ${ }^{10}$ Secondly, people are more motivated by tangible gains such as a financial benefit, than by long-term intangible gains like a reduction in the chance of negative health outcomes. These two irrational 'decision biases' can be used to nudge people toward healthy behavior that is beneficial for them in the long run. ${ }^{11}$ An incentive in the form of money or vouchers can be the immediate, concrete reward which can motivate people to stop smoking.

Several studies have shown that financial incentives for smoking cessation success can be an effective method to stimulate smoking cessation. ${ }^{12-18}$ For instance, incentives have proven to be successful in increasing continuous abstinence in pregnant women, ${ }^{15}$ in homeless smokers, ${ }^{16}$ and in substance abusers. ${ }^{17}$ A long-term study by Volpp et al. ${ }^{18}$ conducted in the United States involved 878 participating employees of a multinational corporation. Participants were given the opportunity to follow behavioral counseling for smoking cessation near their hometown and, if desired, in combination with pharmacological treatment. Both behavioral counseling and medication were fully reimbursed by the employer. Participants in the intervention group received an incentive of $\$ 100$ for completing a smoking cessation program, $\$ 250$ for smoking abstinence at 6 months after study enrollment, and $\$ 400$ for smoking abstinence at 12 months after study enrollment. Participants in the intervention group (with incentive) were significantly more likely to be completely abstinent after 6,12 , and 15 months than in the control group. Furthermore, participants in the intervention group were more likely to have started a smoking cessation program and to have completed the program. While incentives thus can motivate smokers to enroll in a cessation program, only few participants in the Volpp 
study ${ }^{18}$ actually did enroll (incentive group $15.4 \%$ vs control group 5.4\%). Importantly, the rate of quitters was substantially higher in participants from the incentive group who participated in the smoking cessation program than participants from the control group who enrolled in the training (46.3\% vs $20.8 \%$ ). It is therefore likely that the combination of an effective smoking cessation program with incentives for quit success will prove to be the most effective in increasing smoking cessation rates.

The chance to quit successfully increases when people receive professional stop smoking support. ${ }^{19}$ Yet, smoking cessation treatment is relatively expensive for people with low incomes and the cost of medication can be a barrier. ${ }^{20}$ Since a lower income is also related to a higher smoking prevalence, ${ }^{21}$ it is particularly important to make smoking cessation counseling accessible for people with lower incomes. If smoking cessation therapy is fully reimbursed, more smokers will make use of it which can lead to twice as many long term quitters. ${ }^{22,23}$ It is therefore conceivable that smokers are more willing to start a smoking cessation treatment when their employer accounts for the costs. For a smoking cessation training combined with incentives to be widely adopted by commercial companies as a common smoking cessation intervention, it needs to be cost-effective. To our knowledge, no study that has investigated the effect of incentives on long-term smoking abstinence in a workplace setting has incorporated an economic evaluation. We are only aware of a randomized controlled trial in pregnant women which found that incentives of 400 pounds were highly cost-effective. ${ }^{24}$ Smoking cessation training in combination with incentives might therefore be attractive to employers in light of employee health improvement but also from a cost saving perspective.

The proposed "Continuous Abstinence Through Corporate Healthcare" (CATCH) is conducted in Dutch companies. Currently, about a quarter of Dutch adults smoke. Smoking prevalence is much higher among low (28\%) and moderate educated adults (27\%) than among high educated adults (19\%). ${ }^{25}$ This socioeconomic difference has been increasing over time in the Netherlands. ${ }^{26}$ The higher prevalence of smoking in individuals from lower socioeconomic groups is the single most important cause of socioeconomic differences in mortality. ${ }^{27,28}$ In order to decrease these differences, it is vital to specifically target the lower socioeconomic population.

The aim of this study is to investigate whether financial incentives combined with a smoking cessation training can improve quit success. In addition, an economic evaluation is conducted to assess the costs and benefits of this intervention. The results of this study can provide key evidence for the applicability and effectiveness of incentives for smoking cessation and can offer recommendations for implementing incentives to reduce employee smoking in a corporate context. 


\section{METHODS}

The primary aim of this study is to evaluate whether adding a financial incentive (compared with no additional incentive) is effective and cost-effective in increasing 12-month continuous abstinence rates in tobacco smoking employees participating in a smoking cessation group training. A secondary aim is to investigate the effect of the incentive on the quit rate immediately after the training, after three and after six months. Furthermore, this study investigates the cost-effectiveness of the incentive in terms of quit rate and utilities, both from a societal perspective and from the employer's perspective. A cluster-randomized controlled trial is conducted in 44 Dutch companies who offer their employees an evidence-based treatment for smoking cessation. Participants from the intervention companies receive incentives when smoking abstinence is achieved at a fixed time schedule (Figure 1), whereas participants from the control companies do not receive incentives (care as usual). 


\begin{tabular}{|c|c|c|c|}
\hline & Recruitment of companies & \\
\hline & & $\begin{array}{l}\text { Communication of study to } \\
\text { employees }\end{array}$ & \\
\hline & & $\begin{array}{l}\text { Inclusion of employees and } \\
\text { informed consent }\end{array}$ & \\
\hline & & Randomization of companies & \\
\hline \multicolumn{2}{|c|}{ Intervention companies } & & Control companies \\
\hline \multicolumn{2}{|c|}{$\begin{array}{l}\text { T0 questionnaire (immediately } \\
\text { before the smoking cessation } \\
\text { training) }\end{array}$} & & $\begin{array}{l}\text { T0 questionnaire (immediately } \\
\text { before the smoking cessation } \\
\text { training) }\end{array}$ \\
\hline \multicolumn{2}{|c|}{$\begin{array}{l}\text { Smoking cessation training } \\
\text { (including weekly CO measurement } \\
\text { session 2-7) }\end{array}$} & & $\begin{array}{l}\text { Smoking cessation training } \\
\text { (including weekly CO measurement } \\
\text { session 2-7) }\end{array}$ \\
\hline \multicolumn{2}{|c|}{$\begin{array}{l}\text { T1 questionnaire and biochemical } \\
\text { validation (immediately after the } \\
\text { smoking cessation training) }\end{array}$} & & $\begin{array}{l}\text { T1 questionnaire and biochemical } \\
\text { validation (immediately after the } \\
\text { smoking cessation training) }\end{array}$ \\
\hline $\begin{array}{l}\text { ABSTINENT = } \\
\text { voucher } € 50\end{array}$ & $\begin{array}{l}\text { NOT ABSTINENT = } \\
\text { No incentive }\end{array}$ & & No incentive \\
\hline \multicolumn{2}{|c|}{$\begin{array}{l}\text { T2 questionnaire and biochemical } \\
\text { validation ( } 3 \text { months after the } \\
\text { counseling sessions) }\end{array}$} & & $\begin{array}{l}\text { T2 questionnaire and biochemical } \\
\text { validation ( } 3 \text { months after the } \\
\text { counseling sessions) }\end{array}$ \\
\hline $\begin{array}{l}\text { ABSTINENT = } \\
\text { voucher } € 50\end{array}$ & $\begin{array}{l}\text { NOT ABSTINENT = } \\
\text { No incentive }\end{array}$ & & No incentive \\
\hline \multicolumn{2}{|c|}{$\begin{array}{l}\text { T3 questionnaire and biochemical } \\
\text { validation ( } 6 \text { months after the } \\
\text { counseling sessions) }\end{array}$} & & $\begin{array}{l}\text { T3 questionnaire and biochemical } \\
\text { validation ( } 6 \text { months after the } \\
\text { counseling sessions) }\end{array}$ \\
\hline $\begin{array}{l}\text { ABSTINENT = } \\
\text { voucher } € 50\end{array}$ & $\begin{array}{l}\text { NOT ABSTINENT = } \\
\text { No incentive }\end{array}$ & & No incentive \\
\hline \multicolumn{2}{|c|}{$\begin{array}{l}\text { T4 questionnaire and biochemical } \\
\text { validation ( } 12 \text { months after the } \\
\text { counseling sessions) }\end{array}$} & & $\begin{array}{l}\text { T4 questionnaire and biochemical } \\
\text { validation ( } 12 \text { months after the } \\
\text { counseling sessions) }\end{array}$ \\
\hline $\begin{array}{l}\text { ABSTINENT }= \\
\text { voucher } € 200\end{array}$ & $\begin{array}{l}\text { NOT ABSTINENT }= \\
\text { No incentive }\end{array}$ & & No incentive \\
\hline
\end{tabular}

Figure 1. Flow-chart of design and measurements 


\section{Study population}

Approximately 516 tobacco smoking employees, randomized from 22 intervention companies and 22 control companies, participate in this study. To be eligible for participating in the study, companies have to meet certain criteria: (1) the management is willing to pay for the counseling sessions and (2) the management agrees that employees participate in the counseling sessions and carbon monoxide (CO) measurements during working hours or directly after on a location arranged by the company. In order to be eligible to participate in this study, an employee needs to be a current tobacco smoker and at least 18 years old. At the request of the employer, spouses who smoke are allowed to enroll in the study. Subjects are not eligible for participation in this study when they have an acute life-threatening disease, are not able to read or speak the Dutch language or have already started an attempt to quit smoking at the moment of inclusion.

\section{Recruitment}

In order to recruit companies, SineFuma - an organization that provides smoking cessation group training to companies - presents their clients with the possibility of participating in this research project. Additionally, the research assistant actively approaches companies by telephone and via a recruitment letter sent by post or e-mail with a visually attractive flyer attached. Press releases, media interviews and social media (Facebook, LinkedIn, Twitter) are used to create awareness of the study, and advertisements are placed in digital newsletters of several organizations, such as the Dutch Cancer Society and Lung Alliance Netherlands. Companies are targeted based on number of employees ( $n>200)$ and socioeconomic status of employees (preferably lower educated workers). In the recruitment process, companies are informed that they can be randomized to the incentive group or the control group. To which group the company is assigned is revealed during the first session of the smoking cessation training. This means that a company needs to decide to participate in the research without knowing the final result of the randomization. When a company decides to participate in the study, participants within the company are recruited using advertisements via the corporate website, email, posters and informative flyers that are spread through the company building. On these promotion materials it is advertised that participants have a chance to earn financial incentives with a total amount of 350 euro. On request of the company, SineFuma or the research assistant organizes an informal presentation at the company to inform employees who consider participating in the training. Prior to enrolling in the study, employees receive an information letter and are asked to give their signed informed consent. In this information letter, the chance of being eligible to earn the financial incentives is explained, and detailed information on the smoking cessation training and the research procedures is provided.

\section{Intervention}

\section{Smoking cessation counseling}

Evidence-based smoking cessation counseling is provided by experienced trainers of SineFuma to both employees in the intervention companies and the control companies. Groups of approximately 8-16 participants receive counseling in seven sessions of ninety minutes on a location arranged by the company. The training is practical in nature and focuses on ways to quit successfully and how to avoid relapse. It relies on a buddy system 
group dynamic to effectively promote peer support. The trainer informs participants about the possibility to use smoking cessation aids like nicotine replacement therapy and medication during their quit attempt. The company decides whether it reimburses the medication or if their employees have to claim it from their health insurance. After two sessions in which the participants are prepared, they stop smoking at the third session. In session four to seven, the participants receive counseling to get through the first difficult weeks of smoking abstinence. As a standard part of the smoking cessation training, participants perform a CO measurement every week starting the second session, which serves as a tool to motivate and encourage them to stay quit.

\section{Incentives}

As part of the intervention, participants from the intervention group who are continuously abstinent from smoking receive financial incentives. There is a fixed time schedule for the incentives to help maximize the effect on smoking cessation success. At T1, T2, and T3, participants who report to have been abstinent since the end of the smoking cessation training (continuous abstinence) and who have a CO score lower than $10 \mathrm{ppm}^{29}$ at the respective visit receive $€ 50$ credit. At T4, abstinent participants are given credit representing a value of $€ 200$. Participants who did not succeed in quitting smoking at T1 get a second chance to earn a voucher at T2 and to be eligible for the vouchers at T3 and T4 if they are verifiable smoking abstinent at T2 (point prevalence abstinence). The participants receive a digital code by e-mail which they can use to access a digital gift shop where they can exchange their voucher for a broad range of products and activities.

\section{Outcomes}

The primary outcome is carbon monoxide validated 12 months (T1-T4) continuous abstinence from smoking (Russel's standard). ${ }^{29}$ This is evaluated by self-report of smoking and validated by biochemical measurements. Self-report of smoking abstinence is assessed by measuring seven-day point prevalence abstinence and prolonged abstinence ${ }^{29}$ and biochemically verified using a CO measurement. Abstinence is defined as smoking not more than five cigarettes from the start of the abstinence period. Secondary outcomes are smoking abstinence immediately after smoking cessation counseling (T1), after three months (T2) and after six months (T3). The same methods and definitions to determine smoking abstinence are used as for the primary outcome.

\section{Carbon monoxide measurement}

Smoking abstinence is biochemically validated by measuring expired air carbon monoxide concentrations using a cut-off point of 9 parts per million among those who report having quit smoking using a handheld monitor ( $\mathrm{PiCO}^{\mathrm{TM}}$ Smokerlyzer, Bedfont Scientific Ltd., Kent, England). This is a non-invasive and reliable method to detect recent smoking. ${ }^{29}$ As a part of the smoking cessation training, participants will perform a CO measurement in session 2-7. After the training, CO measurements will be performed by the research assistant to validate smoking abstinence. The validation takes place at the company within two weeks after distribution of the questionnaire at T1, T2, T3, and T4. When there is discordance between self-report of smoking abstinence and biochemical validation or when participants do not cooperate with biochemical validation, participants are assumed to be smokers. ${ }^{29}$ 


\section{Questionnaires}

Participants fill out web-based questionnaires about their smoking and cessation behavior, use of resources (health care and other societal costs), and generic quality of life at T0 - T4. At T0, participants are asked about demographic characteristics, current and past smoking (cessation) behavior, number of pack years smoked, ${ }^{30}$ quit intention, use of smoking cessation treatment, and nicotine dependence. ${ }^{31}$ At T1, participants are asked to evaluate the counseling sessions via multiple choice questions concerning the quality of the training and the coach, the individual components of the training, and the fact that the training was organized within the company. At T1 - T4, self-report of smoking abstinence is assessed by measuring seven-day point prevalence abstinence and prolonged abstinence. ${ }^{29}$ At T4, participants from the intervention group are asked to evaluate the incentives via five multiple choice questions about appreciation of the incentives and their perceived effectiveness. At all measurement points, participants are asked about quit intention, use of smoking cessation treatment, nicotine dependence, stress, attitudes, self-efficacy, risk perception, (peer) social support, smoking regulations at the workplace, medical care and medication use.

\section{Process evaluation}

A process evaluation, consisting of questionnaires and interviews, is conducted to assess participants' experience with the study. At 6 months after the smoking cessation training, qualitative interviews are conducted with participants from the intervention companies who quit smoking successfully and participants from the intervention companies who did not. At least 15 interviews are conducted, after which the point of data saturation will be reached if in three further consecutive interviews no new themes or relationships between themes have emerged. ${ }^{32}$ From the participants who did not manage to quit, participants are interviewed who did not accomplish smoking abstinence in the early phase during the training, and participants who relapsed later on. The aim of the interviews is to gain insight in the participants' motivation to enroll in the training, to hear their opinion on the effect of the incentive and to inquire their appraisal of the program. The interviews are recorded, transcribed and coded using NVivo software. Coding is performed by two independent researchers. Discrepancies are discussed with a third researcher. Researchers involved in interviewing and data analysis keep a diary to evaluate their own subjective views on the interpretation of the data.

\section{Sample size calculation}

The study from Volpp et al. ${ }^{18}$ is used to obtain estimates for the sample size calculation. After 12 months, $15 \%$ of participants in the intervention group and $5 \%$ in the control group were abstinent.Withanalphaof0.05, therequired samplesizetoobtainthisclinicallyrelevanteffect size with $80 \%$ power is 141 participants per treatment group, based on the Chi-square test. Since the randomization is on cluster (company) level and assuming a mean number of participants per company $(\mathrm{m})$ of 12 and an intra-class correlation (ICC) of 0.05 [31], the design effect $1+(\mathrm{m}-1)$ ICC $=1.55$, yields a sample size of 219 participants per group. Taking into account $15 \%$ loss to follow up due to unexpected employee turnover, ${ }^{18} 516$ participants (44 companies) have to be included in the study in total. 


\section{Randomization}

Participants are allocated via cluster-randomization on a company level to the intervention or control group. For large corporations with several branches in which the participants are not in direct contact with each other, each location is entered separately in the randomization. The randomization procedure is performed by an independent research assistant. The sequence of the randomization is generated with a digital randomization program using the biased urn method, ${ }^{33,34}$ where the proportion of being randomized into a group is inversely related with the proportion of participants who are already randomized into that group, in order to maintain treatment balance throughout the trial.

\section{Statistical analysis}

Based on the intention-to-treat principle, all randomized participants are included in the denominator for calculating abstinence rates with the exception of unavoidable loss to follow-up as stated the Russel Standard. ${ }^{29}$ The primary effectiveness analysis examines the difference in prolonged smoking abstinence between intervention group and control group over a period of twelve months after the counseling sessions. To be able to account for repeated measures and nesting of participants within companies, generalized mixed models with the logit link are used. Time, group (intervention or control) and the interaction between time and group are entered in the model as fixed factors. Baseline measurements such as socioeconomic status (based on income and education) and nicotine dependence are considered as potential effect modifiers and will therefore be explored by moderation in the analysis. If those are indeed effect-modifiers, the effects for each level of socioeconomic status and nicotine dependence are reported. In case of missing values in these potential effect modifiers, multiple imputation approach is used. Missing data in the outcome variable are not being imputed, since the likelihood-based approach is used to deal with missing values..$^{35} \mathrm{~A}$ two-tailed test is considered statistically significant with $\mathrm{p}$-values $<0.05$.

\section{Economic evaluation}

An economic evaluation in the form of a cost-effectiveness (CEA) and cost-utility analysis (CUA) from a societal perspective and from an employer's perspective ${ }^{36}$ is embedded in this trial-based economic evaluation. The time horizon and the measurement point are combined with the effectiveness study, i.e. the T0-T4 measurement. The primary outcome for the CEA is cost per continuously abstinent ex-smoker. The primary outcome of the CUA is cost per Quality Adjusted Life Year (QALYs). The utility value derived from the standard quality of life questionnaire, EuroQol 5D5L, ${ }^{37}$ using Dutch tariff ${ }^{38}$ will be used to compute QALYs. The utilities at the various time points are used to compute QALYs by means of the area under the curve method..$^{39}$ As this economic evaluation is also performed from a societal perspective, this implies that all relevant costs and outcomes are considered. A separate analysis is performed from the employer's perspective. It is hypothesized that the intervention is associated with an increased number of quitters, increase in quality of life, and decreased costs. The time horizon is 12 months. Costs (the use of resources) are measured continuously; outcomes for the economic evaluation study are measured before the start of the smoking cessation training, at 3 months, 6 months, and 12 months. 


\section{Cost measurement}

Total costs are estimated using a bottom-up (or micro-costing) approach, where information on each element of service used is multiplied by an appropriate unit cost and summed to provide an overall total cost. Intervention costs, healthcare costs, respondent and family costs, and costs outside the health care sector are assessed, especially the cost for the employer. A cost questionnaire was especially designed for this study, based on existing questionnaires, ${ }^{40-43}$ which identifies all relevant costs aspects. Subjects are asked to report the data from their cost questionnaire relating to the previous 3 months at T0, and relating to the period in between measurements at T1-T4. The valuation of costs is based mainly on the updated Dutch manual for cost analysis. ${ }^{44}$ Cost prices are expressed in 2017 euros. If necessary, existing cost-prices are updated to 2017 using the consumer price index.

\section{Analysis of economic evaluation}

The analysis of the economic evaluation contains several uncertainty analyses, including bootstrapping analysis for sample uncertainty. The results of the economic evaluation are presented in cost-effectiveness plane and cost-effectiveness acceptability curves (CEAC). The Incremental cost-effectiveness ratio (ICER) is determined on the basis of incremental costs and effects of evidence-based interventions for smoking cessation in a corporate setting compared to care as usual. The cost-effectiveness ratio is stated in terms of costs per outcomerate,thecost-utilityratiofocusesonthenetcostperQualityAdjustedLifeYeargained. The ICER is calculated as follows. ICER $=(\mathrm{Ci}-\mathrm{Cc}) /(\mathrm{Ei}-\mathrm{Ec})$, where $\mathrm{Ci}$ is the annual total cost of the evidence-based interventions for smoking cessation in a company setting group, $\mathrm{Cc}$ is the annual total cost of the care as usual group, Ei is the effects at one year follow-up for the evidence-based interventions for smoking cessation in a company setting group and Ec is the effect at one year follow-up for the care as usual group. The robustness of the ICER is checked by non-parametric bootstrapping. Bootstrap simulations are also conducted in order to quantify the uncertainty around the ICER, yielding information about the joint distribution of cost and effect differences. The bootstrapped cost-effectiveness ratios are subsequently plotted in a cost-effectiveness plane, in which the vertical line reflects the difference in costs and the horizontal line reflects the difference in effectiveness. The choice of treatment depends on the maximum amount of money that society is prepared to pay for a gain in effectiveness, which is called the ceiling ratio. Therefore, the bootstrapped ICERs are also depicted in a cost-effectiveness acceptability curve showing the probability that evidence-based interventions for smoking cessation in a business setting is cost-effective using a range of ceiling ratios. Additionally, to demonstrate the robustness of our base-case findings, a multi-way sensitivity analysis is performed. In the sensitivity analysis, uncertain factors of assumptions in the base case analysis are recalculated in order to assess whether the assumptions have influenced the incremental cost-effectiveness ratio (ICER), for example by varying cost-prices and volumes between minimum and maximum. ${ }^{45}$ 


\section{DISCUSSION}

This paper presents the protocol of the intervention study "Continuous AbstinenceThrough Corporate Healthcare" (CATCH). The aim of the study is to investigate the effectiveness of incentives on continuous abstinence in employees. A total of 516 employees, divided over 22 control and 22 intervention companies participate in a cluster-randomized controlled trial. In both groups, participants follow a seven-week smoking cessation program. In the intervention group, employees additionally receive vouchers for cessation success up to a total value of 350 euro. It is hypothesized that employees in the incentive group achieve a higher 12-month continuous abstinence rate.

\section{Strengths and limitations}

The proposed study has several strengths, starting with its company setting. A company setting can have several advantages compared to other settings; corporations have the potential to reach a large amount of people, can motivate their employees to participate and thereby achieve high attendance rates, and may inspire peer support and positive peer pressure among colleagues. ${ }^{46}$ Moreover, businesses have an interest in keeping their employees healthy. ${ }^{4}$

Another strong point of this study is that the proposed study performs a cost-effectiveness and cost-utility analysis from a societal perspective and specifically from an employer's perspective. To our best knowledge, the cost-effectiveness of financial incentives to stimulate long term smoking cessation in a company setting has not been established yet. The outcomes of the cost-effectiveness analysis can help inform employers who are considering providing smoking cessation support in combination with incentives for their employees.

What makes this study also particularly relevant is its target population of employees with a lower socioeconomic status. People with a lower education and income smoke more often ${ }^{25,47}$ smoke more cigarettes per day and have lower quit rates than people with a higher SES. ${ }^{26,48}$ Although smokers with low SES are as likely to start a quit attempt as smokers with a high SES, they are less likely to succeed in quitting smoking. ${ }^{49}$ Potential barriers in quitting successfully are suggested to be lack of social support and financial barriers to smoking cessation treatment. ${ }^{47}$ The current intervention aims to remove potential obstacles and to make the smoking cessation program easily accessible for employees in three ways: (1) by providing it via the employer, (2) by organizing the training sessions at the workplace, and (3) by designing the training as a group program so that colleagues can support each other both during and between sessions.

A possible limitation of this study is the lack of blinding of the research participants to the existence of the intervention and control conditions. It is expected that the incentive group is more attractive to subjects than the control group. If smokers would know for certain that the incentive is within reach, it could lead to employees signing up who only smoke occasionally and are not dependent on the training for a successful quit attempt. In contrast, the control group may appeal only to heavy smokers who are highly motivated to quit. If nothing would be arranged to avoid these potential problems, it was 
anticipated that the study would end up with a substantial larger number of subjects in the intervention condition than in the control condition, which could cause selection bias. To avoid this possibility, it was decided not to randomize participants prior to enrolment, but to inform them merely about their $50 \%$ chance of being eligible to earn the vouchers. Only after enrolment, during the first training session, it is revealed whether participants are randomized into the control or intervention condition. This approach could, however, lead to attrition bias, when participants in the control group are disappointed about not being able to earn the vouchers and therefore drop out more frequently. Furthermore, it is anticipated that some larger companies will participate with more than one group. In order to avoid inequality among coworkers, it was decided that all groups within the same location of the corporation would be randomized together. A disadvantage of this decision is that when these groups do not start at the same time, it could cause selection bias, since employees in the second group of the company may find out whether they will receive the incentive or not.

\section{Conclusion}

The proposed study is the first in the Netherlands that investigates the effect of financial rewards on smoking cessation and the first study worldwide that assesses the costeffectiveness of incentives to decrease smoking in employees. The results of this study can provide important recommendations for the use of financial incentives to motivate employees to quit smoking. 


\section{REFERENCES}

1. US Department of Health and Human Services. The health consequences of smoking-50 years of progress. In. a report of the Surgeon General. Vol 17. Atlanta, GA: US Department of Health and Human Services, Centers for Disease Control and Prevention, National Center for Chronic Disease Prevention and Health Promotion, Office on Smoking and Health; 2014.

2. Jha P, Ramasundarahettige C, Landsman V, et al. 21st-Century Hazards of Smoking and Benefits of Cessation in the United States. New England Journal of Medicine. 2013;368(4):341-350.

3. Xu X, Bishop EE, Kennedy SM, Simpson SA, PechacekTF. Annual healthcare spending attributable to cigarette smoking: an update. Am J Prev Med. 2015;48.

4. Berman M, Crane R, Seiber E, Munur M. Estimating the cost of a smoking employee. Tobacco Control. 2014;23(5):428-433.

5. Halpern MT, Shikiar R, Rentz AM, Khan ZM. Impact of smoking status on workplace absenteeism and productivity. Tobacco Control. 2001;10(3):233-238.

6. CBS. Gezondheid en zorg in cijfers. Voorburg: Centraal Bureau voor de Statistiek;2007.

7. Weng SF, Ali S, Leonardi-Bee J. Smoking and absence from work: systematic review and metaanalysis of occupational studies. Addiction. 2013;108(2):307-319.

8. Eriksen MP, Gottlieb NH. A review of the health impact of smoking control the workplace. American journal of health promotion : AJHP. 1998;13(2):83-104.

9. Osinubi OY, Slade J. Tobacco in the workplace. Occupational medicine (Philadelphia, Pa). 2002;17(1):137-158, vi.

10. O'Donoghue T, Rabin M. Doing it now or later. American Economic Review. 1999:103-124.

11. Loewenstein $\mathrm{G}$, Brennan $\mathrm{T}$, Volpp KG. ASymmetric paternalism to improve health behaviors. JAMA. 2007;298(20):2415-2417.

12. Giles EL, Robalino S, McColl E, Sniehotta FF, Adams J. The Effectiveness of Financial Incentives for Health Behaviour Change: Systematic Review and Meta-Analysis. PLoS ONE. 2014;9(3):e90347.

13. Haff N, Patel MS, Lim R, et al. The Role of Behavioral Economic Incentive Design and Demographic Characteristics in Financial Incentive-Based Approaches to Changing Health Behaviors: A MetaAnalysis. American Journal of Health Promotion. 2015;29(5):314-323.

14. Cahill K, Hartmann-Boyce J, Perera R. Incentives for smoking cessation. Cochrane Database of Systematic Reviews. 2015(5).

15. Tappin D, Bauld L, Purves D, et al. Financial incentives for smoking cessation in pregnancy: randomised controlled trial. BMJ. 2015;350:h134.

16. Businelle MS, Kendzor DE, Kesh A, et al. Small financial incentives increase smoking cessation in homeless smokers: A pilot study. Addictive Behaviors. 2014;39(3):717-720.

17. Shoptaw $S$, Rotheram-Fuller $E$, Yang $X$, et al. Smoking cessation in methadone maintenance. Addiction. 2002;97(10):1317-1328.

18. Volpp KG, Troxel AB, Pauly MV, et al. A Randomized, Controlled Trial of Financial Incentives for Smoking Cessation. New England Journal of Medicine. 2009;360(7):699-709.

19. Hartmann-Boyce J, Stead LF, Cahill K, Lancaster T. Efficacy of interventions to combat tobacco addiction: Cochrane update of 2012 reviews. Addiction. 2013;108(10):1711-1721.

20. Giskes K, Kunst AE, Ariza C, et al. Applying an Equity Lens to Tobacco-Control Policies and Their Uptake in Six Western-European Countries. Journal of Public Health Policy. 2007;28(2):261-280.

21. Huisman M, Kunst AE, Mackenbach JP. Inequalities in the prevalence of smoking in the European Union: comparing education and income. Preventive Medicine. 2005;40(6):756-764. 
22. Kaper J, Wagena EJ, Willemsen MC, Van Schayck CP. Reimbursement for smoking cessation treatment may double the abstinence rate: results of a randomized trial. Addiction. 2005;100(7):1012-1020.

23. Reda AA, Kotz D, Evers SM, van Schayck CP. Healthcare financing systems for increasing the use of tobacco dependence treatment. The Cochrane Library. 2012.

24. Boyd KA, Briggs AH, Bauld L, Sinclair L, Tappin D. Are financial incentives cost-effective to support smoking cessation during pregnancy? Addiction. 2015.

25. Verdurmen J, Monshouwer K, Van Laar M, Van Bon-Martens M. Factsheet Continu Onderzoek Rookgewoonten 2013. Utrecht: Trimbos-instituut;2014.

26. Nagelhout GE, de Korte-de Boer $D$, Kunst $A E$, et al. Trends in socioeconomic inequalities in smoking prevalence, consumption, initiation, and cessation between 2001 and 2008 in the Netherlands. Findings from a national population survey. BMC public health. 2012;12(1):1-9.

27. Strand $\mathrm{BH}$, Tverdal $\mathrm{A}$. Can cardiovascular risk factors and lifestyle explain the educational inequalities in mortality from ischaemic heart disease and from other heart diseases? 26 year follow up of 50000 Norwegian men and women. Journal of Epidemiology and Community Health. 2004;58(8):705-709.

28. Stringhini $S$, Sabia $S$, Shipley $M$, et al. Association of socioeconomic position with health behaviors and mortality. JAMA. 2010;303(12):1159-1166.

29. West RPLJ. Outcome criteria in smoking cessation trials: proposal for a common standard. Addiction. 2005;100(3):299-303.

30. Janjigian YY, McDonnell K, Kris MG, et al. Pack-years of cigarette smoking as a prognostic factor in patients with stage IIIB/IV nonsmall cell lung cancer. Cancer. 2010;116(3):670-675.

31. Kozlowski LT, Porter CQ, Orleans CT, Pope MA, Heatherton T. Predicting smoking cessation with self-reported measures of nicotine dependence: FTQ, FTND, and HSI. Drug and Alcohol Dependence. 1994;34(3):211-216.

32. Francis $\mathrm{JJ}$, Johnston $\mathrm{M}$, Robertson $\mathrm{C}$, et al. What is an adequate sample size? Operationalising data saturation for theory-based interview studies. Psychology \& Health. 2010;25(10):1229-1245.

33. Schouten HJ. Adaptive biased urn randomization in small strata when blinding is impossible. Biometrics. 1995;51(4):1529-1535.

34. Efron B. Forcing a sequential experiment to be balanced. Biometrika. 1971;58(3):403-417.

35. Little RJ, Rubin DB. Statistical analysis with missing data. John Wiley \& Sons; 2014.

36. Noben C, Evers S, Nieuwenhuijsen K, et al. Protecting and promoting mental health of nurses in the hospital setting: Is it cost-effective from an employer's perspective? International journal of occupational medicine and environmental health. 2015;28(5):891-900.

37. Group TE. EuroQol-a new facility for the measurement of health-related quality of life. Health policy. 1990;16(3):199-208.

38. Versteegh MM, Vermeulen KM, Evers SM, de Wit GA, Prenger R, Stolk EA. Dutch Tariff for the FiveLevel Version of EQ-5D. Value in health. 2016.

39. Drummond MF, Sculpher MJ, Claxton K, Stoddart GL, Torrance GW. Methods for the economic evaluation of health care programmes. Oxford university press; 2015.

40. Thorn J, Coast J, Cohen D, et al. Resource-Use Measurement Based on Patient Recall: Issues and Challenges for Economic Evaluation. Appl Health Econ Health Policy. 2013;11(3):155-161.

41. Bouwmans $C$, Krol M, Severens $H$, Koopmanschap M, Brouwer W, Roijen LH-v. The iMTA Productivity Cost Questionnaire: A Standardized Instrument for Measuring and Valuing HealthRelated Productivity Losses. Value in Health. 2015;18(6):753-758. 
42. Bouwmans $C$, Hakkaart-van Roijen L, Koopmanschap M, Krol M, Severens H, Brouwer W. Medical Cost Questionnaire (iMCQ). In: Rotterdam: iMTA, Erasmus Universiteit Rotterdam; 2013.

43. Dirum. Database of instruments for resource use management. www.dirum.org. Published 2016. Accessed.

44. Tan SS, Bouwmans CAM, Rutten FFH, Hakkaart-van Roijen L. UPDATE OF THE DUTCH MANUAL FOR COSTING IN ECONOMIC EVALUATIONS. International Journal of Technology Assessment in Health Care. 2012;28(02):152-158.

45. Briggs $A H$, Wonderling DE, Mooney CZ. Pulling cost-effectiveness analysis up by its bootstraps: A non-parametric approach to confidence interval estimation. Health economics. 1997;6(4):327-340.

46. Cahill K, Lancaster T. Workplace interventions for smoking cessation. The Cochrane Library. 2014.

47. Hiscock R, Bauld L, Amos A, Fidler JA, Munafò M. Socioeconomic status and smoking: a review. Annals of the New York Academy of Sciences. 2012;1248(1):107-123.

48. Reid JL, Hammond D, Boudreau C, Fong GT, Siahpush M. Socioeconomic disparities in quit intentions, quit attempts, and smoking abstinence among smokers in four western countries: findings from the International Tobacco Control Four Country Survey. Nicotine \& tobacco research : official journal of the Society for Research on Nicotine and Tobacco. 2010;12 Suppl:S20-33.

49. Kotz D, West R. Explaining the social gradient in smoking cessation: it's not in the trying, but in the succeeding. Tob Control. 2009;18(1):43-46. 

CHAPTER 5

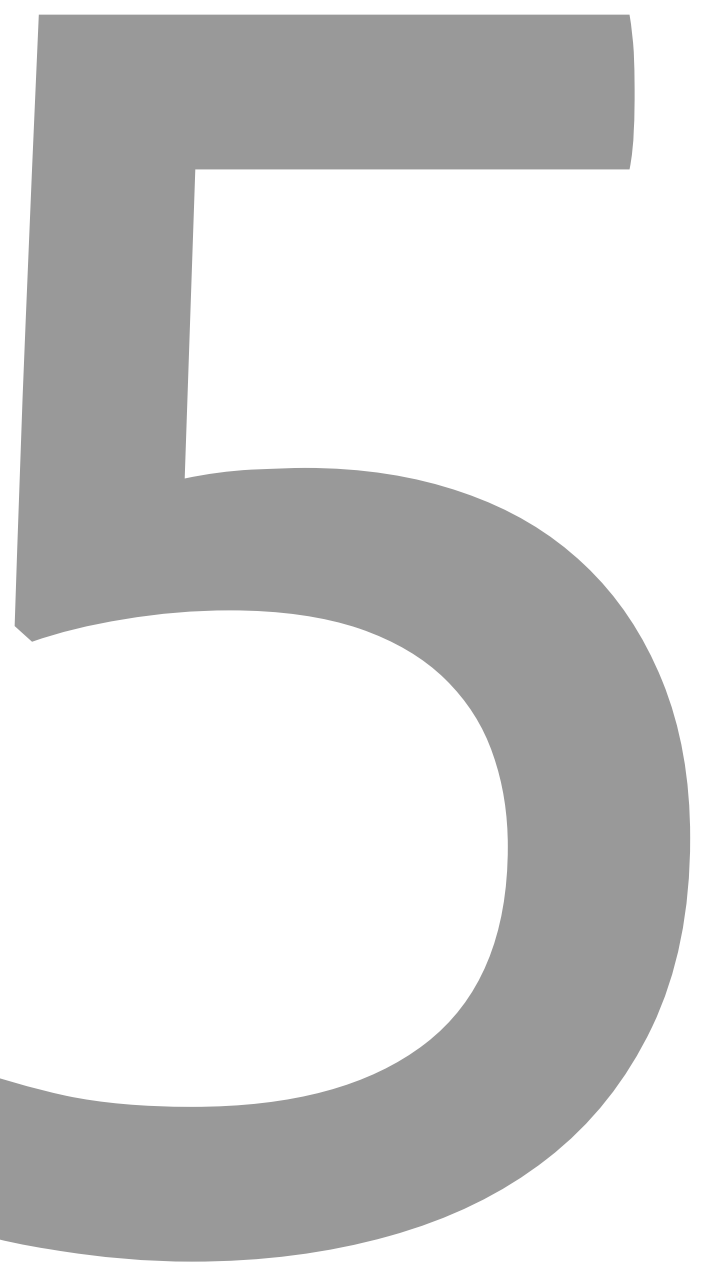




\section{Effect of a workplace-based group training program combined with financial incentives on smoking cessation: a cluster-randomized controlled trial}

Van den Brand FA, Nagelhout GE, Winkens B, Chavannes NH, van Schayck OCP. Effect of a workplace-based group training program combined with financial incentives on smoking cessation: a cluster-randomized controlled trial 


\section{ABSTRACT}

Background Financial incentives are potentially useful tools to aid smoking cessation, but robust evidence to support their efficacy, particularly in combination with other interventions (e.g., group counselling), has not been reported. We aimed to investigate whether financial incentives combined with a smoking cessation group training program (compared with a training program with no incentives) organized at the workplace would increase 12-month abstinence rates in tobacco-smoking employees with different education and income levels.

Methods This cluster-randomized controlled trial was done in the Netherlands with companies that offer a smoking cessation group training program to all of their smoking employees. Eligible participants were tobacco-smoking employees and spouses of employees who were at least 18 years of age. Participants in the control group received a weekly 90-min session of smoking cessation group training for 7 weeks at the workplace; in addition to the group training, participants in the intervention group received vouchers for being abstinent ( $€ 50$ at the end of the training program, $€ 503$ months after completion of the program, $€ 50$ after 6 months, and $€ 200$ after 12 months). Companies were randomly assigned by an independent research assistant to the intervention group or the control group with a digital randomization program, using a biased urn method. The primary outcome was carbon monoxide-validated continuous abstinence at 12 months. All randomized participants were included in the modified intention-to-treat analyses, with the exception of unavoidable loss (participants who had died or moved to an untraceable address (according to the Russell Standard), and in the sensitivity analyses, except the complete case analysis, which included only participants for whom all variables included in the model were not missing. This study is registered with the Dutch Trial Register, number NTR5657.

Findings Between March 1, 2016, and March 1, 2017, 61 companies with 604 participating smokers were enrolled. 31 companies (319 smokers) were randomly assigned to the intervention group and 30 companies ( 285 smokers) to the control group. 12 months after finishing the smoking cessation program, the proportion of individuals abstaining from smoking in the intervention group was significantly higher than that in the control group (131 [41\%] of 319 vs 75 [26\%] of 284 ; adjusted odds ratio $1.93,95 \% \mathrm{Cl} 1.31-2.85, \mathrm{p}=0.0009$; adjusted for education level, income level, and Fagerström score).

Interpretation Financial incentives in addition to a smoking cessation group training program can significantly increase long-term smoking abstinence. The results of the current study could motivate employers to facilitate a workplace smoking cessation program with financial incentives to help employees to quit smoking. 


\section{INTRODUCTION}

Financial incentives have shown to be effective in increasing smoking cessation rates in diverse populations. ${ }^{1-3}$ Reviews on the effect of incentives showed moderate effects of incentives on smoking cessation. ${ }^{2-4}$ However, the quality of the evidence is low because of inadequate randomization and allocation procedures, deficient outcome reporting and confounding. ${ }^{2}$ Furthermore, a wide variety in type and size of the incentives impairs drawing conclusions on their effectiveness. Additionally, previous studies were mainly done in the USA, which might limit generalizability. 2,3 Finally, most studies have solely investigated the effect of incentives, without accompanying group counselling for smoking cessation..$^{5-7}$ The effect of the combination of incentives with counselling is especially important to assess, considering evidence that group counselling can effectively enhance quit success. ${ }^{8}$

Previous studies provided an indication of which aspects of incentive-based programs are effective. A study comparing different incentive schemes showed that rewardbased incentive programs (smokers receive a reward) can possibly be more effective than deposit-based programs (smokers receive a refund of their own money deposit) in increasing smoking cessation, because of relatively high acceptance rates of rewardbased incentive programmes. ${ }^{9}$ Additionally, group counselling is more effective than selfhelp or less intensive help, such as brief support from a healthcare provider. ${ }^{8}$

The workplace can be a constructive environment to stimulate tobacco cessation ${ }^{10}$ and promotion of tobacco cessation is financially beneficial for employers. Employees who smoke tobacco show increased workplace absenteeism, loss of productivity due to smoking breaks or withdrawal symptoms, and increased healthcare cost. ${ }^{11-13}$ Moreover, exsmokers show decreased absenteeism and productivity losses compared with continuing smokers within 1-4 years. ${ }^{11}$ For employees, a smoking cessation training program at the workplace is easily accessible, because it does not require a financial contribution, does not cost extra travel time, and it is situated in a familiar environment. Furthermore, group counselling with colleagues can have an extra advantage of social support and peer pressure during and between counselling sessions. ${ }^{10,14}$

In the Netherlands in 2017, 23\% of individuals aged 18 years and older among the general population smoked on a regular basis. ${ }^{15}$ However, smoking prevalence is much higher among people with a low level of education (defined as elementary school and lower secondary education) in the Netherlands (29\%) than among people with a high level of education (18\%; defined as upper secondary education and university), and this difference in smoking prevalence is also found between people with lower and higher incomes. ${ }^{15}$ Smoking among employees strongly varies between work settings and can be substantially higher within certain companies. ${ }^{16}$ Since 2004 , by government decree, all employees are entitled to a smoke-free workplace and smoking in public places is prohibited; however, many companies have special rooms or cabins for smoking inside their building, or sheltered smoking areas on the company premises. 
Although incentives for smoking cessation have been studied in different ways, these studies show modest quit rates at best. Although previous studies have shown evidence that reward-based incentives, group counselling and workplace situated interventions are effective, ${ }^{5,8,10}$ to our knowledge no study has effectively combined these elements. The aim of the current study was to examine the effectiveness of a combination of these effective components within a cluster-randomized trial. It was hypothesized that incentives in combination with a group smoking cessation training program organized at the workplace would increase quit rates compared with a group training program alone. $A$ second aim was to investigate whether incentives might result in different cessation rates for employees with different levels of education, income, or nicotine dependency.

\section{METHODS}

\section{Study design}

The design of this study is a cluster-randomized controlled trial among Dutch companies who offer a smoking cessation group training program to all their smoking employees. The protocol of this study with a detailed description of the methods has been published, ${ }^{17}$ and can be consulted for additional information regarding research methods. The study protocol was approved by the Medical Research Ethics Committee of Atrium Medical Centre Heerlen, Heerlen, Netherlands (number 16-N-13).

\section{Participants}

We approached companies of varying size and from different industry types in the Netherlands to participate in this study by email and phone. Companies were required to facilitate a smoking cessation training program at the workplace during or directly after working hours. Employees within participating companies were recruited by the company management using flyers, posters, email, and intranet messages, and spouses could also participate. Participants needed to be current tobacco smokers aged at least 18 years. Exclusion criteria were an acute life-threatening disease, not being a currently active smoker, not being able to read or speak Dutch, and having already started an attempt to quit smoking at the moment of inclusion. Before the start of the study, all participants were informed about the design of the study and the possibility to receive vouchers for quit success. All participants gave written informed consent

\section{Randomization and masking}

Participants were randomly assigned on a cluster level to the intervention or control group. Participants from the intervention companies received financial incentives of up to $€ 350$ for successful smoking cessation, whereas participants from the control companies did not receive financial incentives. A cluster was defined as the assembly of participants within a company who followed the smoking cessation training program together as a group. By cluster randomization, all participants within a group were allocated to the same (intervention or control) group to prevent possible feelings of unfairness. The randomization sequence was generated by a digital program using the biased urn method, in order to maintain allocation to intervention groups as balanced as possible. ${ }^{18}$ The randomization program was written by a statistician (BW), but companies were 
randomly allocated by an independent research assistant not involved in the study. Group allocation was not revealed to participants or employers until the start of the first training session.

Because of the nature of the intervention, it was not possible to mask the outcome assessor for the intervention groups. However, during the statistical analyses, which were first done at the 6-month follow-up and repeated at the 12-month follow-up, all researchers, including the statistician, were masked to treatment allocation. Allocation was unblinded after the first analyses were completed and the research team unanimously agreed on the interpretation of the findings.

\section{Procedures}

A smoking cessation group training program was organized at each of the participating companies. The training program consisted of a 90-min session per week for 7 weeks. The training sessions were given by professional coaches from the Dutch company SineFuma, which is experienced in giving smoking cessation group training in a workplace setting. The pre-existing training program was designed to help participants to initiate a quit attempt and guide them through the first few difficult weeks of quitting smoking, with an important role for group dynamics and peer support. Participants quit together at the start of the third session and had quit smoking for about 1 month at the last session.

Participants in the intervention group could earn four vouchers with a total worth of $€ 350$. The first $€ 50$ voucher was received on the condition of validated smoking abstinence at the end of the smoking cessation training program. The second and third $€ 50$ vouchers could be earned when participants were abstinent three and six months after finishing the cessation program. At the end of the study (12 months post cessation program), participants could earn an additional $€ 200$ voucher. ${ }^{17}$ The amount of the incentives was chosen to be high enough to motivate quitting smoking, while remaining acceptable for employers to implement in the future. The payment schedule was designed so that the first reward was received relatively soon, i.e. directly after finishing the training, and so that the reward that was given after the longest period of abstinence (12 months) was the largest, to be attractive to the participants from the start of the intervention taking into account the typical discounting of delayed rewards. ${ }^{19}$ The vouchers were sent by email in the form of a digital code which could be exchanged via a web shop for a large variety of products or activities.

\section{Outcomes}

The primary outcome was 12-month carbon monoxide (CO) validated continuous smoking abstinence according to the Russell Standard. ${ }^{20}$ Smoking abstinence was assessed by selfreport of continuous abstinence, biochemically validated by $\mathrm{CO}$ measurement. Secondary outcomes were CO-validated smoking abstinence and self-reported abstinence directly after completing the smoking cessation training program, after three months, and after six months. A PiCO ${ }^{\mathrm{TM}}$ Smokerlyzer (Bedfont Scientific Ltd., Kent, England) was used to measure expired-air $\mathrm{CO}$ concentrations, with a cut-off point of 9 parts per million. ${ }^{20}$ In case of disagreement between participants' self-report of smoking and CO measurement, participants were classified as smokers. Missing values on self-reported smoking and 
missing $\mathrm{CO}$ validation on endpoint measurements were counted as smokers. That is, if an individual self-reported or was found to be smoking at 3 or 6 months but was abstinent at the 12-month visit, they would not receive the $€ 200$ financial incentive and would not be classified has having met the primary endpoint.

We used a baseline questionnaire to assess participant characteristics. Participants were asked to state their highest completed education, which was recoded into three categories: low (none completed, primary school and lower secondary education), moderate (middle secondary education) and high (upper secondary education and university). We assessed net monthly household income with a multiple-choice item with 13 categories. Income was individualized for each participant by taking the mean of the lower and upper boundary of each household income category and dividing this amount by the square root of the number of people in the household ${ }^{21}$ The individualized income was divided into three categories based on tertiles. Furthermore, we assessed whether participants had attempted to quit smoking in the past (yes or no), and how many cigarettes on average they smoked per day. We calculated pack-years to assess lifetime smoking and we measured nicotine dependence with the Fagerström Nicotine Addiction questionnaire, in which a scale from 1 (not addicted) to 10 (heavily addicted) determines the level of nicotine addiction. ${ }^{22}$

\section{Statistical analysis}

Estimates for the sample size calculation were based on a previous study with $15 \%$ quitters in the intervention group versus $5 \%$ in the control group. ${ }^{5}$ With an alpha of 0.05 and $80 \%$ power to detect this effect, a sample size of 141 participants per group was required. Considering the cluster-randomized design and assuming an average of 12 participants per cluster (m), and an intra-class correlation (ICC) of $0.05,{ }^{23}$ the design effect $(=1+(m-1)$ ICC $)$ equals 1.55 , which yielded a sample size of 219 participants per group. Considering $15 \%$ loss to follow up due to unexpected employee turnover ${ }^{5}$ at least 516 participants within at least 44 clusters needed to be included in the study. We present participant characteristics using frequencies (\%) for categorical variables and means (SDs) for numerical variables. In the modified intention-to-treat analysis, all randomly assigned participants were included in the denominator for calculating abstinence with the exception of unavoidable loss (participants who had died or moved to an untraceable address), as stated in the Russell Standard. ${ }^{20}$ We assessed the difference in continuous smoking abstinence (within 1, 3, 6, or 12 months) between the intervention group and the control group using a generalized linear mixed-effects model analysis with a logit link (binary outcome) and a random intercept at the company level to adjust for the clustering of participants within a company. The fixed part of the model included group (intervention vs control) and prespecified variables known to be related to the outcome: income (three categories), education (three categories), and nicotine dependency (Fagerström score). We imputed missing data on these variables using the other fixed variables that were included in the model, and used sex, company, number of attended sessions (0-7), and continuous abstinence as predictors. We used multiple imputation to create 50 complete datasets, where the maximum number of iterations was set to 20 and trace lines were used to check convergence. We used the complete case analysis (including only participants for whom all variables included in the model were not missing), as well as the model that 
only included group as the fixed factor (no correction for other variables), as sensitivity analyses. We investigated the possible effect modification of income, educational level, and nicotine dependency (Fagerström score) by including these factors as interactions with group in the model. Two-sided $p$ values of 0.05 or less were considered statistically significant. We used IBM SPSS Statistics for Windows (version 23.0) to compute descriptive statistics. We used the RStudio version 1.1.383 mice package for multiple imputation and Ime4 package (glmer function) for the generalized linear mixed-effects models.

\section{RESULTS}

61 companies were recruited from March 1, 2016, to March 1, 2017 (table 1). 694 smokers were contacted, of whom four did not meet the inclusion criteria, 50 were not able or did not want to participate in the study, and 36 did not provide a signed informed consent form (figure 1). The remaining 604 participants (of whom one was a spouse) were randomly assigned to the control group (30 clusters, 285 participants) or the intervention group (31 clusters, 319 participants), and used as the denominator in all analyses according to the modified intention-to-treat protocol, with the exception of the biochemically validated outcome at 12 months, where one participant from the control group was excluded from the analysis because of unavoidable loss to follow-up (figure 1). The mean age of the participants was 45 years (SD 10.17), and the majority were men (table 2). 481 (80\%) of 604 participants reported having tried to quit in the past (table 2). Participants reported a mean of 22 pack-years (SD 13.17) and a mean Fagerström score of 4.4 (1.98; table 2). The mean net annual individualized household income in our study was $€ 24$ 170.97 (SD 8788.53; appendix), which makes the $€ 350$ incentive $1.45 \%$ of the participants' average annual household income. 
Table 1. Company characteristics

\begin{tabular}{|c|c|c|}
\hline Characteristics of companies & $\begin{array}{c}\text { Intervention } \\
\text { group }(\mathrm{n}=\mathbf{3 1})\end{array}$ & $\begin{array}{c}\text { Control group } \\
(\mathbf{n}=\mathbf{3 0 )}\end{array}$ \\
\hline \multicolumn{3}{|l|}{ Company size, n (\%) } \\
\hline $0-50$ & $2(6 \%)$ & $2(7 \%)$ \\
\hline $51-100$ & $2(6 \%)$ & $3(10 \%)$ \\
\hline $101-200$ & $3(10 \%)$ & $4(13 \%)$ \\
\hline $201-500$ & $8(26 \%)$ & $5(17 \%)$ \\
\hline $501-1000$ & $9(29 \%)$ & $4(13 \%)$ \\
\hline$>1000$ & $7(23 \%)$ & $12(40 \%))$ \\
\hline \multicolumn{3}{|l|}{ Industry type ${ }^{a}$} \\
\hline Administrative and support service activities & $3(10 \%)$ & 0 \\
\hline Agriculture, Forestry and Fishing & $1(3 \%)$ & 0 \\
\hline Education & $3(10 \%)$ & $1(3 \%)$ \\
\hline Financial and insurance activities & $2(6 \%)$ & $2(7 \%)$ \\
\hline Human health and social work activities & $4(13 \%)$ & $8(27 \%)$ \\
\hline Manufacturing & $7(23 \%)$ & $9(30 \%)$ \\
\hline Mining and Quarrying & $1(3 \%))$ & 0 \\
\hline Professional, scientific and technical activities & 0 & $2(7 \%)$ \\
\hline Public administration and defense; compulsory social security & $2(6 \%)$ & $3(10 \%)$ \\
\hline Real estate activities & 0 & $1(3 \%)$ \\
\hline Transportation and storage & $4(13 \%)$ & $2(7 \%)$ \\
\hline Water supply, sewerage, waste management and remediation activities & $1(3 \%)$ & $2(7 \%)$ \\
\hline Wholesale and retail trade & $3(10 \%)$ & 0 \\
\hline \multicolumn{3}{|l|}{$\begin{array}{l}\text { Company smoking regulations } \mathbf{s}^{\mathbf{b}} \\
\text { Smoking is allowed }\end{array}$} \\
\hline $\begin{array}{l}\text { Smoking is allowed } \\
\text { Yes }\end{array}$ & $254 / 319(80 \%)$ & $236 / 285(83 \%)$ \\
\hline No & $51 / 319(16 \%)$ & $38 / 285(13 \%)$ \\
\hline Missing & $14 / 319(4 \%)$ & $11 / 285(4 \%)$ \\
\hline \multicolumn{3}{|l|}{ Employees may take a smoking break whenever they like } \\
\hline Yes & $174 / 319(55 \%)$ & $188 / 285(66 \%)$ \\
\hline No & $130 / 319(41 \%)$ & $86 / 285(30 \%)$ \\
\hline Missing & $15 / 319(5 \%)$ & $11 / 285(4 \%)$ \\
\hline Cluster size, mean (SD) & $10.6(3.5)$ & $10.4(3.6)$ \\
\hline
\end{tabular}

Data are $\mathrm{n}(\%)$ of companies, $\mathrm{n} / \mathrm{N}$ (\%) of employees, or mean (SD).

${ }^{a}$ Based on the Standard Industrial Classification.

${ }^{\mathrm{b}}$ Based on self-report of individual employees, since possibilities to take a smoking break could vary depending on the type of job the employee had within a company. 


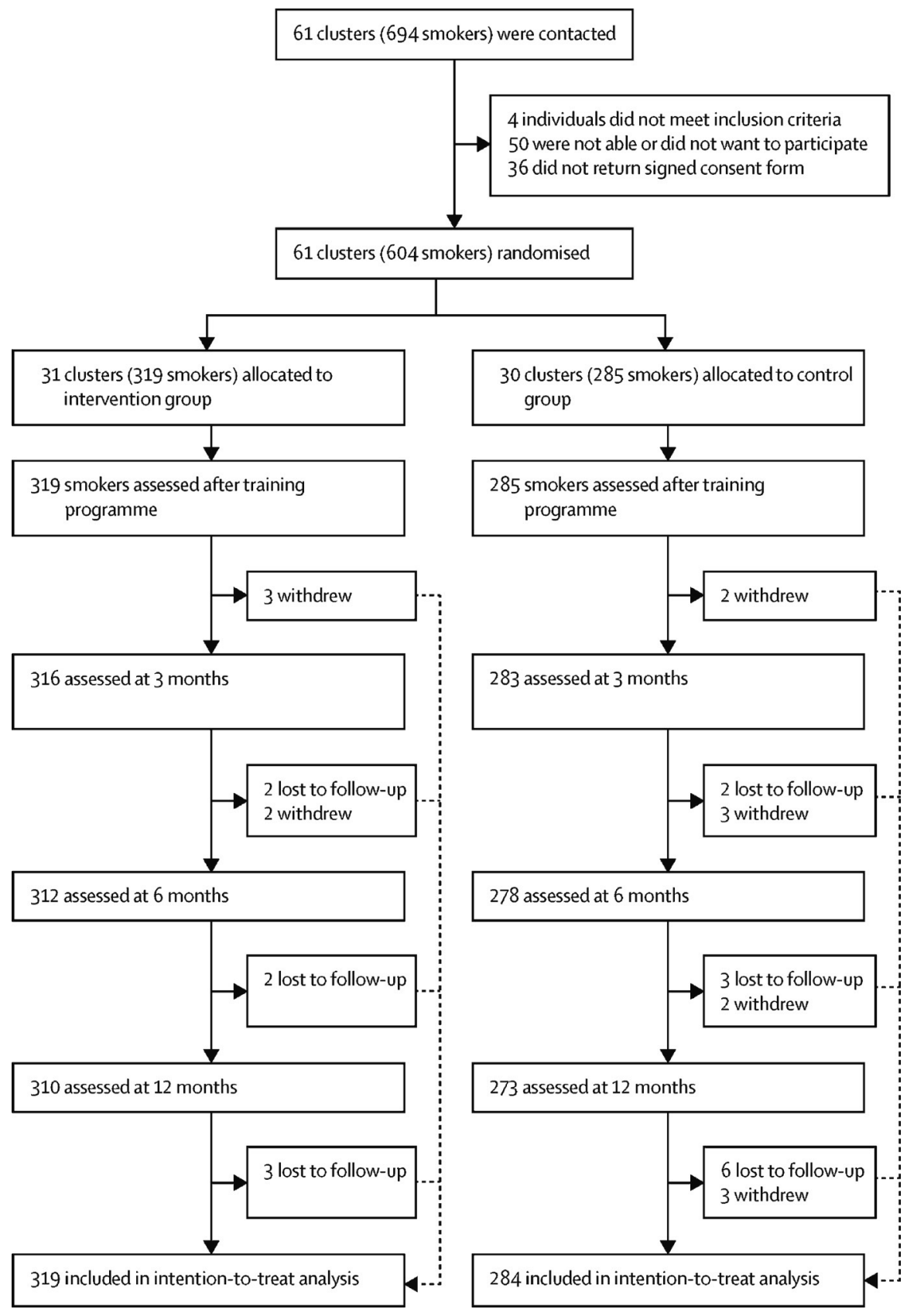

Figure 1. Trial profile 
The smoking cessation training program consisted of seven sessions, of which participants attended a mean of 5.5 sessions (SD 1.6) in the control group and a mean of 5.8 sessions (1.4) in the intervention group (appendix). Participants from both groups who were abstinent from smoking at 12 months after the training program had attended, on average, about one session more than participants who had relapsed to smoking.

In all cases in which participants self-reported that they were abstinent and $\mathrm{CO}$ verification was done, the $\mathrm{CO}$ score confirmed the participants' self-report (figure 2; table 3). For the primary outcome at 12 months, 131 (41\%) of 319 participants in the intervention group and $75(26 \%)$ of 284 participants in the control group were verified quitters (adjusted odds ratio [OR] $1.93,95 \% \mathrm{Cl} 1.31-2 \cdot 85 ; \mathrm{p}=0.0009$; table 3 ). Directly after completion of the smoking cessation training program, $266(83 \%)$ of 319 participants in the intervention group and $216(76 \%)$ of 285 participants in the control group were verified quitters (adjusted OR 1.77, 95\% Cl 1.00-3.12; $\mathrm{p}=0.050$; table 3). At 3 months, the proportion of quitters was 172 (54\%) of 319 in the intervention group and 125 (44\%) of 285 in the control group (adjusted OR 1.55, 95\% Cl 1.07 to 2.24; $\mathrm{p}=0.021$; table 3 ). At 6 months, the difference in the proportion of participants who were abstinent between the intervention group and control group increased to $19 \%$ (adjusted OR 2.39, 95\% Cl 1.62-3.52; $p<0.0001$; table 3).

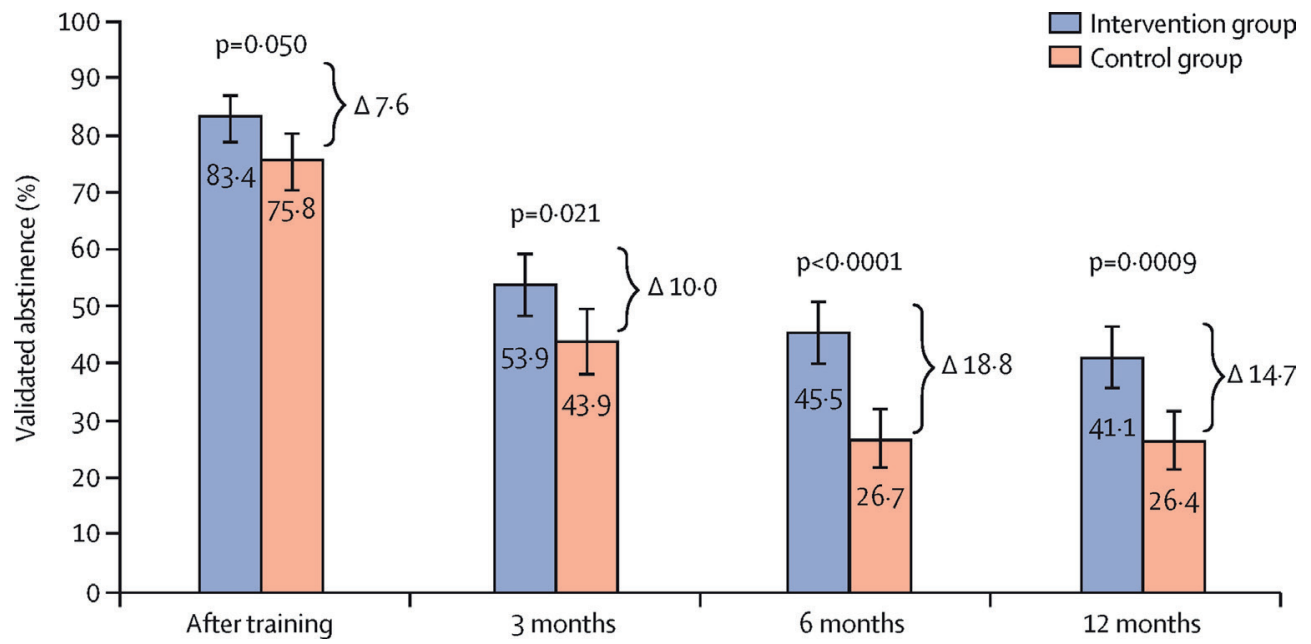

Figure 2. Validated smoking abstinence in the intervention vs control groups

Figure shows histogram of modified intention-to-treat analysis with multiple imputation, adjusted for education level, income level, and Fagerström score, with percentages of validated abstinence in the intervention (smoking cessation program plus financial incentive) and control (smoking cessation program only) groups directly after the smoking cessation training program, at 3 months, 6 months, and 12 months. $\Delta=$ risk difference.

The analyses investigating possible effect modification of income, education, or nicotine dependency showed no significant interactions (all $p$ values $\geq 0.079$; appendix), indicating similar effects for these subgroups. The results for the lowest income and education groups show similar patterns to the whole group; that is, an intervention effect was obtained after 6 months (table 4). 
Table 2. Participant baseline characteristics

\begin{tabular}{|c|c|c|}
\hline & $\begin{array}{l}\text { Intervention group } \\
(\mathrm{n}=319)\end{array}$ & $\begin{array}{l}\text { Control group } \\
(n=285)\end{array}$ \\
\hline Age, years & $43.9(10.4)$ & $46.6(9.7)$ \\
\hline $\begin{array}{l}\text { Sex } \\
\text { Women } \\
\text { Men }\end{array}$ & $\begin{array}{l}102(32 \%) \\
217(68 \%)\end{array}$ & $\begin{array}{l}121(42 \%) \\
164(58 \%)\end{array}$ \\
\hline $\begin{array}{l}\text { Educational level } \\
\text { Low } \\
\text { Middle } \\
\text { High } \\
\text { Missing }\end{array}$ & $\begin{array}{l}97(30.4) \\
136(42.6) \\
75(23.5) \\
11(3.4)\end{array}$ & $\begin{array}{l}62(22 \%) \\
119(42 \%) \\
90(32 \%) \\
14(5 \%)\end{array}$ \\
\hline $\begin{array}{l}\text { Income level } \\
\text { Low } \\
\text { Middle } \\
\text { High } \\
\text { Missing }\end{array}$ & $\begin{array}{l}111(35 \%) \\
91(29 \%) \\
76(24 \%) \\
41(13 \%)\end{array}$ & $\begin{array}{c}68(24 \%) \\
84(29 \%) \\
105(37 \%) \\
28(10 \%)\end{array}$ \\
\hline $\begin{array}{l}\text { Attempted to quit in the past } \\
\text { yes } \\
\text { No } \\
\text { Missing }\end{array}$ & $\begin{array}{c}253(79 \%) \\
55(17 \%) \\
11(3 \%)\end{array}$ & $\begin{array}{c}228(80 \%) \\
48(17 \%) \\
9(3 \%)\end{array}$ \\
\hline $\begin{array}{l}\text { Cigarettes per day } \\
10 \text { or less } \\
11 \text { to } 20 \\
21 \text { to } 30 \\
31 \text { or more } \\
\text { Missing }\end{array}$ & $\begin{array}{l}58(18.2) \\
179(56.1) \\
59(18.5) \\
9(2.8) \\
14(4.4)\end{array}$ & $\begin{array}{l}55(19 \%) \\
159(56 \%) \\
58(20 \%) \\
3(1 \%) \\
10(4 \%)\end{array}$ \\
\hline $\begin{array}{l}\text { Pack years } \\
\text { Missing }\end{array}$ & $\begin{array}{l}21.6(13.2) \\
24(8 \%)\end{array}$ & $\begin{array}{l}23.5(13.0) \\
11(4 \%)\end{array}$ \\
\hline $\begin{array}{l}\text { Fagerström Test of Nicotine Dependence } \\
\text { Missing }\end{array}$ & $\begin{array}{l}4.4(1.9) \\
19(6 \%)\end{array}$ & $\begin{array}{l}4.5(2.0) \\
12(4 \%)\end{array}$ \\
\hline $\begin{array}{l}\text { Used NRT during quit attempt } \\
\text { Yes } \\
\text { No } \\
\text { Missing }\end{array}$ & $\begin{array}{c}134(42 \%) \\
156(49 \%) \\
29(9 \%))\end{array}$ & $\begin{array}{c}130(46 \%) \\
117(41 \%) \\
38(13 \%)\end{array}$ \\
\hline $\begin{array}{l}\text { Used medications* during quit attempt } \\
\text { Yes } \\
\text { No } \\
\text { Missing }\end{array}$ & $\begin{array}{c}77(24 \%) \\
212(66 \%) \\
30(9 \%)\end{array}$ & $\begin{array}{c}43(15 \%) \\
204(72 \%) \\
38(13 \%)\end{array}$ \\
\hline $\begin{array}{l}\text { Used e-cigarettes during quit attempt } \\
\text { Yes } \\
\text { No } \\
\text { Missing }\end{array}$ & $\begin{array}{c}52(16 \%) \\
235(74 \%) \\
32(10 \%)\end{array}$ & $\begin{array}{c}58(20 \%) \\
184(65 \%) \\
43(15 \%)\end{array}$ \\
\hline
\end{tabular}

Data are mean (SD) or $\mathrm{n}(\%)$.

* Varenicine, buproprion, or other smoking cessation medications.

In the sensitivity analysis (appendix) in which only study group was included as the fixed factor, with no correction for other variables, ORs and corresponding $p$ values were in the same direction and of similar magnitude as in the main analysis. The results of the adjusted $(n=507)$ complete case analysis that included only participants for whom none of the variables in the model were missing also showed results similar in size and direction to those of the main analysis (12-month CO-validated abstinence complete case analysis OR 2.14; main analysis OR 1.93; appendix). The intra-class correlation in the CO-validated adjusted complete case analysis was 0.043 for the 12-month measurement point (appendix). 


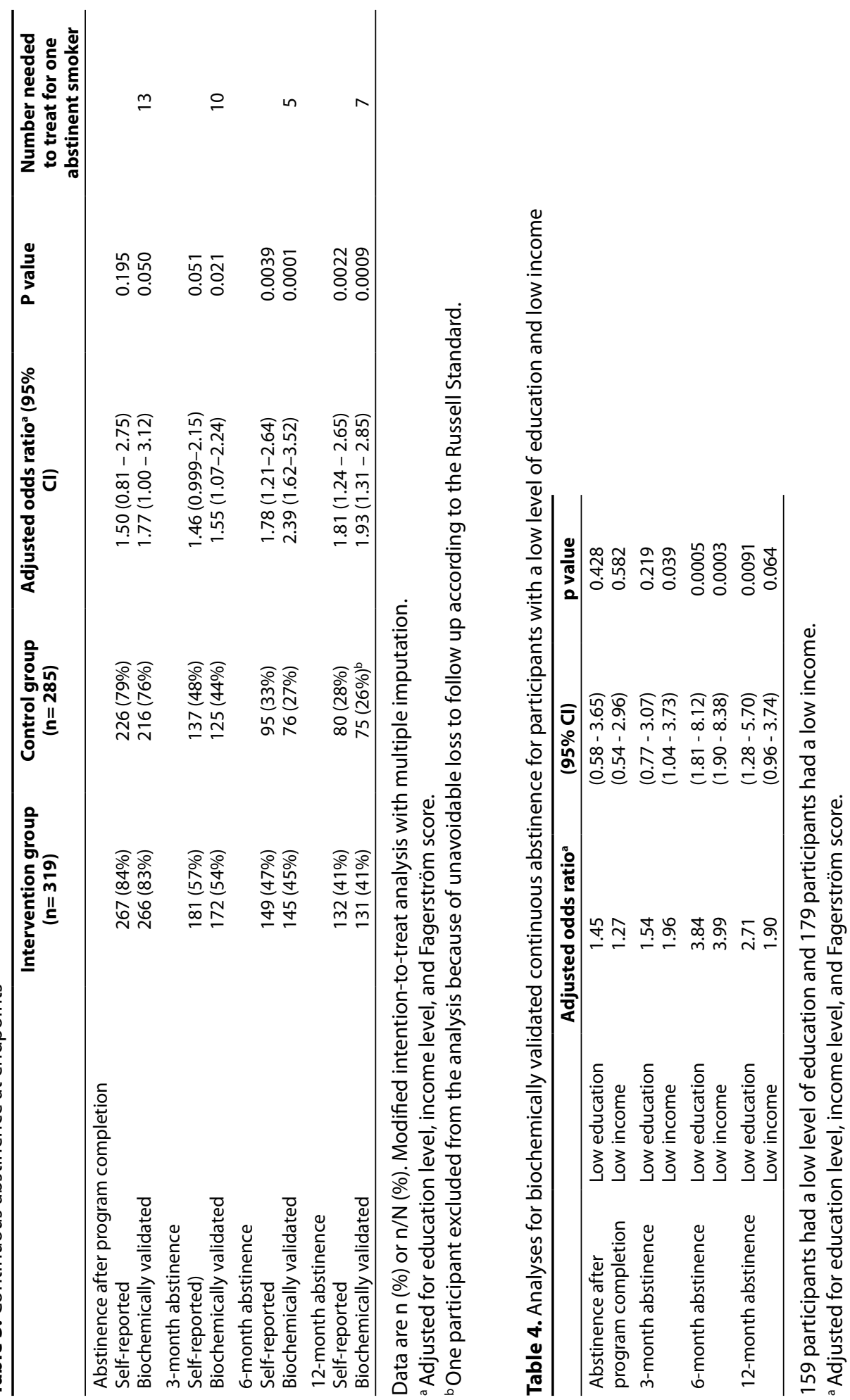




\section{DISCUSSION}

In this cluster-randomized controlled trial, we aimed to determine whether financial incentives in addition to a smoking cessation group training program (compared with a training program with no incentives) was effective in increasing abstinence in tobaccosmoking employees. Our findings show that financial incentives for abstinence in combination with a tobacco cessation group training program at the workplace stimulate smoking cessation and are effective in smokers with a low level of education and income. Incentives can increase the number of successful quitters compared with a group training program alone.

Incentives significantly increased 12-month continuous abstinence, with validated quit percentages of $41 \%$ in the intervention group with $26 \%$ in the control group. In two similar, large studies ${ }^{5,7}$ that investigated incentives for smoking cessation within companies, 12-month quit rates were considerably lower than in the current study. In one of those studies, ${ }^{5} 12$-month quit rates were $9.4 \%$ in the incentive group and $3.6 \%$ in the control group, even though the amount of money used as an incentive in that study (US\$750) was considerably higher than in the current study ( $€ 350$, which is approximately $\$ 410)$. Although participants in that study ${ }^{5}$ were encouraged with a $\$ 100$ incentive to seek smoking cessation counselling, counselling was not a standard part of the protocol, and few people participated in a counselling program (15.4\% in the incentive group and $5.4 \%$ in the control group). Therefore, the higher quit rates observed in the current study than in other workplace-based incentive studies 5,7 might be explained by the addition of the smoking cessation program, in which all participants of the current study were enrolled. Although the current study was not designed to assess the effectiveness of the training program, and quit success might also result from factors outside the training program, motivation to quit smoking elicited by the incentive is likely to increase quit success if smokers are supported in a professional group training program that increases knowledge and skills, provides social support, and removes barriers to quitting. This notion is supported by the observation that, in the current study, the control group (no incentives) also showed high quit rates at 3 months (44\%), while in the control group of the study by Volpp and colleagues ${ }^{5}$ only $11.8 \%$ of participants were abstinent at the first measurement. These numbers might reflect the effectiveness of a comprehensive group training program with multiple sessions, as was used in the current study, and are in line with earlier research that showed the value of smoking cessation counselling and its contribution to successful quitting., ${ }^{8,24}$ Another similar study done in Switzerland investigated the effect of large incentives up to $\$ 1650$ (without counselling) for 12-month abstinence. ${ }^{6}$ The results showed 12 -month abstinence rates of $9.5 \%$ in the incentive group (and $3.7 \%$ in the control group). The current study used much smaller incentives but had higher quit rates than the study of Etter and Schmid, ${ }^{6}$ which suggests that modest incentives might have the potential to be as effective as large incentives if combined with additional smoking cessation support. 
This study showed that the effect of incentives on smoking abstinence was similar among smokers with high, medium, and low levels of education and income, indicating that the intervention was effective even for the low income and education groups. This result is important, because smokers with low socioeconomic status are less likely to quit, ${ }^{25}$ and many existing smoking cessation interventions are more effective among those from higher socioeconomic status groups, ${ }^{26}$ which leads to increasing differences in smoking prevalence. ${ }^{27}$ Therefore, financial incentives for smokers with low socioeconomic status could be an effective strategy to decrease the socioeconomic gap in smoking.

Although most participants in this study were initially successful in quitting smoking during the smoking cessation training program, quit rates rapidly declined within the first 3 months after the training program had finished. Steep relapse curves are also found in smokers who quit without assistance, where most relapses occur in the first 8 days of a quit attempt. ${ }^{28}$ The results showed that the proportion of participants who were abstinent remained fairly stable between the 6 and 12 months of follow-up in both groups, which is in line with research suggesting that the risk of relapse declines to almost zero after 100 days of continuous abstinence..$^{29}$ Importantly, in the present study, the difference in abstinence between the intervention and control group increased over time (from about $8 \%$ to about $15 \%$ ), which shows that incentives are not only effective in motivating initial quit attempts, but can also be a crucial factor for sustained abstinence. The results of this study suggest that professional support can help to lengthen a quit attempt, but raises the question about whether smoking cessation guidance should be extended to a longer period, with, for example, a refresher session within the first 6 months after finishing the smoking cessation program. The right incentive size and timing of delivery might flatten the relapse curve, but research in this area is scarce. ${ }^{2,30,31}$ Therefore, future studies should determine the optimal size and timing of incentives to facilitate quitting, by varying incentive schemes and amounts.

The current study has some limitations that should be taken into consideration. Although this study was executed within a large number of diverse companies, the results might not be generalizable to every company, since employee characteristics might affect outcomes. ${ }^{16}$ Participants volunteered to follow the smoking cessation training program and were therefore assumed to be motivated and ready to quit. Participants in the control group were not masked to the intervention condition of receiving vouchers for quit success. This had the advantage that both groups could be recruited with an identical strategy, which prevented selection bias between the intervention and control group while using the incentives to increase enrolment. ${ }^{32}$ However, this might have led to disappointment or loss of motivation when the randomization result was revealed during the first training session. However, only a small number of participants withdrew from the study in either study group. Furthermore, the mean number of training sessions attended was similar between the intervention and control groups, with a small (but significant) difference ( 5.8 vs 5.5 sessions). This difference might have been caused by a mediating effect of the incentives; for example, the incentives might have increased the participant's motivation to be present at the training sessions. Another limitation of the current study was that these results show quit rates while the incentives were still in place. More research is needed to determine whether incentives also contribute to long-term 
smoking cessation when the incentives are removed. ${ }^{2}$ Although a considerable effort was made to verify each self-reported quitter with biochemical testing (e.g., by making extra company visits and scheduling home appointments) $\mathrm{CO}$ verification could not be done for every participant. This was mostly caused by scheduling problems, vacations, or illness. According to protocol, the participants were included as smokers in the analysis. Since participants in the intervention group could have been more willing to attend the CO measurement appointments in order to receive the incentive, this could explain the small difference between self-reported and biochemically validated quit rates. In the current study, expired air CO measurement was chosen as biochemical verification. Although CO is the preferred method to detect recent smoking, ${ }_{1}^{20}$ there is no objective way of verifying abstinence over the full 12 months. A particular concern with incentive-based trials is that the opportunity to receive a reward in combination with the relatively short detection period of $\mathrm{CO}$ measurement might enable gaming (in which participants deliberately mislead study investigators about their smoking behavior to obtain financial incentives). A previous study ${ }^{33}$ involving financial incentives for smoking cessation in pregnant women that investigated gaming by comparing smoking abstinence based on self-report and CO measurement with cotinine measurement found that only $4 \%$ of participants showed false reporting of smoking status. Although gaming in the current study cannot be ruled out completely, the fact that there were no CO measurements that did not correspond with the participant's self-report of abstinence strengthens confidence in the results. Notably, three participants who at first claimed to be abstinent, upon further inquiry, turned out to have a different definition of abstinence from the research criteria; all participants had quit smoking cigarettes, but one participant still smoked cigars, another smoked marijuana, and the third used a hookah to smoke tobacco. This shows that it is important to clarify what is considered as tobacco smoking when assessing self-report of smoking.

The results of the current study could have implications for current tobacco cessation treatment methods. Previous research has shown that proof of effectiveness of incentives is an important contributor to the acceptability of incentives for quitting smoking. ${ }^{34,35}$ The results of the current study might therefore increase support for this relatively new strategy to decrease smoking. The number needed to treat for a single additional quitter during at least 12 months in the current study was seven (table 3), which shows that incentives are a very effective treatment strategy, whereas previous research showed less favorable numbers need to treat for a single additional quitter in case of pharmacotherapy (number needed to treat for varenicline was ten, for bupropion 18, and 23 for nicotine replacement therapy). ${ }^{36}$ This study showed that modest incentives can elicit substantial quit success. The results of the current study might therefore motivate employers to facilitate a smoking cessation program combined with incentives at the workplace to help employees to quit smoking. 


\section{REFERENCES}

1. Haff N, Patel MS, Lim R, et al. The Role of Behavioral Economic Incentive Design and Demographic Characteristics in Financial Incentive-Based Approaches to Changing Health Behaviors: A MetaAnalysis. Am J Health Promot. 2015;29(5):314-323.

2. Cahill K, Hartmann-Boyce J, Perera R. Incentives for smoking cessation. The Cochrane database of systematic reviews. 2015(5):CD004307.

3. Giles EL, Robalino S, McColl E, Sniehotta FF, Adams J. The Effectiveness of Financial Incentives for Health Behaviour Change: Systematic Review and Meta-Analysis. PLoS One. 2014;9(3):e90347.

4. Van den Brand FA, Nagelhout GE, Reda AA, et al. Healthcare financing systems for increasing the use of tobacco dependence treatment. Cochrane Database Syst Rev. 2017(9).

5. Volpp KG, Troxel AB, Pauly MV, et al. A Randomized, Controlled Trial of Financial Incentives for Smoking Cessation. N Engl J Med. 2009;360(7):699-709.

6. Etter J-F, Schmid F. Effects of Large Financial Incentives for Long-Term Smoking CessationA Randomized Trial. J Am Coll Cardiol. 2016;68(8):777-785.

7. Halpern SD, Harhay MO, Saulsgiver K, Brophy C, Troxel AB, Volpp KG. A Pragmatic Trial of E-Cigarettes, Incentives, and Drugs for Smoking Cessation. N Engl J Med. 2018.

8. Stead LF, Carroll AJ, Lancaster T. Group behaviour therapy programmes for smoking cessation. Cochrane Database Syst Rev. 2017(3).

9. Halpern SD, French B, Small DS, et al. Randomized Trial of Four Financial-Incentive Programs for Smoking Cessation. N Engl J Med. 2015;372(22):2108-2117.

10. Cahill K, Lancaster T. Workplace interventions for smoking cessation. The Cochrane Library. 2014.

11. Halpern MT, Shikiar R, Rentz AM, Khan ZM. Impact of smoking status on workplace absenteeism and productivity. Tob Control. 2001;10(3):233-238.

12. Berman M, Crane R, Seiber E, Munur M. Estimating the cost of a smoking employee. Tob Control. 2014;23.

13. Lundborg P. Does smoking increase sick leave? Evidence using register data on Swedish workers. Tob Control. 2007;16(2):114-118.

14. Carroll C, Rick J, Leaviss J, Fishwick D, Booth A. A qualitative evidence synthesis of employees' views of workplace smoking reduction or cessation interventions. BMC Public Health. 2013;13:1095.

15. CBS. Leefstijl en (preventief) gezondheidsonderzoek; persoonskenmerken. 2017.

16. Centers for Disease Control and Prevention (CDC). Current cigarette smoking prevalence among working adults--United States, 2004-2010. MMWR Morbidity and mortality weekly report. 2011;60(38):1305.

17. Van den Brand FA, Nagelhout GE, Winkens B, et al. The effect of financial incentives on top of behavioral support on quit rates in tobacco smoking employees: study protocol of a clusterrandomized trial. BMC Public Health. 2016;16(1):1056.

18. Schouten HJ. Adaptive biased urn randomization in small strata when blinding is impossible. Biometrics. 1995;51(4):1529-1535.

19. Kirby KN, Maraković NN. Delay-discounting probabilistic rewards: Rates decrease as amounts increase. Psychonomic bulletin \& review. 1996;3(1):100-104.

20. West RPLJ. Outcome criteria in smoking cessation trials: proposal for a common standard. Addiction. 2005;100.

21. OECD. What are equivalence scales? http://www.oecd.org/eco/growth/OECD-NoteEquivalenceScales.pdf. Updated 15-12-2017. Accessed 15-12-2017, 2017. 
22. Heatherton TF, Kozlowski LT, Frecker RC, Fagerstrom K-O. The Fagerström Test for Nicotine Dependence: a revision of the Fagerstrom Tolerance Questionnaire. Br J Addict. 1991;86(9):11191127.

23. Kozlowski LT, Porter CQ, Orleans CT, Pope MA, Heatherton T. Predicting smoking cessation with self-reported measures of nicotine dependence: FTQ, FTND, and HSI. Drug Alcohol Depend. 1994;34(3):211-216.

24. Lancaster T, Stead LF. Individual behavioural counselling for smoking cessation. Cochrane Database of Systematic Reviews. 2017(3).

25. US Department of Health and Human Services. The health consequences of smoking- 50 years of progress. In. a report of the Surgeon General. Vol 17. Atlanta, GA: US Department of Health and Human Services, Centers for Disease Control and Prevention, National Center for Chronic Disease Prevention and Health Promotion, Office on Smoking and Health; 2014.

26. Hill S, Amos A, Clifford D, Platt S. Impact of tobacco control interventions on socioeconomic inequalities in smoking: review of the evidence. Tob Control. 2014;23(e2):e89-e97.

27. Nagelhout GE, de Korte-de Boer $D$, Kunst $A E$, et al. Trends in socioeconomic inequalities in smoking prevalence, consumption, initiation, and cessation between 2001 and 2008 in the Netherlands. Findings from a national population survey. BMC Public Health. 2012;12(1):1-9.

28. Hughes JR, Keely J, Naud S. Shape of the relapse curve and long-term abstinence among untreated smokers. Addiction. 2004;99(1):29-38.

29. Kirshenbaum AP, Olsen DM, Bickel WK. A quantitative review of the ubiquitous relapse curve. $J$ Subst Abuse Treat. 2009;36(1):8-17.

30. Higgins ST, Washio Y, Lopez AA, et al. Examining two different schedules of financial incentives for smoking cessation among pregnant women. Prev Med. 2014;68:51-57.

31. Donatelle R, Hudson D, Dobie S, Goodall A, Hunsberger M, Oswald K. Incentives in smoking cessation: status of the field and implications for research and practice with pregnant smokers. Nicotine \& tobacco research : official journal of the Society for Research on Nicotine and Tobacco. 2004;6 Suppl 2:S163-179.

32. Hennrikus DJ, Jeffery RW, Lando HA, et al. The SUCCESS Project: The Effect of Program Format and Incentives on Participation and Cessation in Worksite Smoking Cessation Programs. Am J Public Health. 2002;92(2):274-279.

33. lerfino D, Mantzari E, Hirst J, Jones T, Aveyard P, Marteau TM. Financial incentives for smoking cessation in pregnancy: a single-arm intervention study assessing cessation and gaming. Addiction. 2015;110(4):680-688.

34. Promberger M, Dolan P, Marteau TM. "Pay them if it works": Discrete choice experiments on the acceptability of financial incentives to change health related behaviour. Soc Sci Med. 2012;75(12):2509-2514.

35. Robertson L, Gendall P, Hoek J, Marsh L, McGee R. Perceptions of financial incentives for smoking cessation: a survey of smokers in a country with an endgame goal. Nicotine \& Tobacco Research. 2017:ntx268-ntx268.

36. Van Schayck OCP, Williams S, Barchilon V, et al. Treating tobacco dependence: guidance for primary care on life-saving interventions. Position statement of the IPCRG. NPJ Primary Care Respiratory Medicine. 2017;27:38. 


\section{APPENDIX}

Table 5. Number of attended training sessions in intervention and control group.

\begin{tabular}{lccc}
\hline & $\begin{array}{c}\text { Intervention group } \\
(\mathbf{n = 3 1 9 )}\end{array}$ & $\begin{array}{c}\text { Control group } \\
(\mathbf{n = 2 8 5})\end{array}$ & p-value $^{\mathbf{b}}$ \\
\hline Number of training sessions attended ${ }^{\text {a }}$, mean (SD) & & & \\
Entire sample & $5.8(1.4)$ & $5.5(1.6)$ & 0.028 \\
Participants who were abstinent at 12 months & $6.3(0.9)$ & $6.0(1.3)$ & 0.575 \\
Participants who were smokers at 12 months & $5.4(1.6)$ & $5.3(1.6)$ & 0.064 \\
\hline
\end{tabular}

${ }^{a}$ From a total of 7 training sessions. ${ }^{b}$ t-test.

Table 6. Sensitivity analyses for continuous abstinence after the training, at 3 months, at 6 months and at 12 months.

\begin{tabular}{|c|c|c|c|c|c|c|c|}
\hline & \multicolumn{3}{|c|}{$\begin{array}{l}\text { MI, unadjusted }^{a} \\
n=604\end{array}$} & \multicolumn{3}{|c|}{$\begin{array}{c}\text { CCA, adjusted }^{b} \\
n=527\end{array}$} & \multirow[b]{2}{*}{ ICC } \\
\hline & OR & $(95 \% \mathrm{CI})$ & p-value & OR & $(95 \% \mathrm{CI})$ & p-value & \\
\hline \multicolumn{8}{|l|}{ After training } \\
\hline Self-reported & 1.42 & $(0.77-2.60)$ & 0.262 & 1.59 & $(0.82-3.07)$ & 0.171 & 0.164 \\
\hline Biochemically validated & 1.70 & $(0.96-2.99)$ & 0.068 & 1.81 & $(0.98-3.32)$ & 0.056 & 0.130 \\
\hline \multicolumn{8}{|l|}{3 months } \\
\hline Self-reported & 1.43 & $(0.98-2.10)$ & 0.065 & 1.61 & $(1.04-2.50)$ & 0.033 & 0.054 \\
\hline Biochemically validated & 1.50 & $(1.04-2.17)$ & 0.031 & 1.67 & $(1.10-2.54)$ & 0.015 & 0.037 \\
\hline \multicolumn{8}{|l|}{6 months } \\
\hline Self-reported & 1.77 & $(1.21-2.60)$ & 0.003 & 1.85 & $(1.21-2.83)$ & 0.005 & 0.040 \\
\hline Biochemically validated & 2.32 & $(1.58-3.40)$ & $<0.001$ & 2.31 & $(1.51-3.52)$ & $<0.001$ & 0.029 \\
\hline \multicolumn{8}{|l|}{12 months } \\
\hline Self-reported & 1.82 & $(1.25-2.63)$ & 0.002 & 2.03 & $(1.31-3.16)$ & 0.002 & 0.046 \\
\hline Biochemically validated & 1.93 & $(1.32-2.82)$ & $<0.001$ & 2.14 & $(1.37-3.33)$ & $<0.001$ & 0.043 \\
\hline
\end{tabular}

a Only group as fixed factor

${ }^{\mathrm{b}}$ Adjusted for education, income and Fagerström score

$\mathrm{MI}=$ multiple imputation

$\mathrm{CCA}=$ complete case analysis

ICC $=$ intra class correlation

Table 7. P-values for interactions

\begin{tabular}{lccccc}
\hline & \multicolumn{2}{c}{ Education } & \multicolumn{2}{c}{ Income } & Fagerström score \\
\cline { 2 - 5 } & low vs middle & low vs high & low vs middle & low vs high & \\
\hline After training & 0.378 & 0.932 & 0.532 & 0.251 & 0.765 \\
3 months & 0.734 & 0.652 & 0.356 & 0.524 & 0.478 \\
6 months & 0.317 & 0.079 & 0.115 & 0.191 & 0.359 \\
12 months & 0.586 & 0.135 & 0.968 & 0.943 & 0.240 \\
\hline
\end{tabular}


Table 8. Participant baseline characteristics

\begin{tabular}{lc}
\hline Characteristics of participants & $\begin{array}{c}\text { Intervention group + control group } \\
\text { (n = 604) }\end{array}$ \\
\hline Age, years & $45(10 \cdot 2)$ \\
Sex & \\
Women & $223(36 \cdot 9)$ \\
Men & $381(63 \cdot 1)$ \\
Attempted to quit in the past & \\
yes & $481(79 \cdot 6)$ \\
No & $103(17 \cdot 1)$ \\
Missing (\%) & $20(3 \cdot 3)$ \\
Pack-years & $22.5(13 \cdot 2)$ \\
Missing & $35(5 \cdot 8)$ \\
Fagerström Test of Nicotine Dependence & $4 \cdot 4(2 \cdot 0)$ \\
Missing & $31(5 \cdot 1)$ \\
Individualized household income, $€$ & $24170(8789)$ \\
Missing & $69(11 \cdot 4)$ \\
\hline
\end{tabular}

Data are mean (SD) or $\mathrm{n}(\%)$. 
CHAPTER 6 


\section{'Secretly, it's a competition': a qualitative study investigating what helped employees quit smoking during a workplace smoking cessation group training program with incentives}

Van den Brand FA, Dohmen LME, Van Schayck OCP, Nagelhout GE. 'Secretly, it's a competition': a qualitative study investigating what helped employees quit smoking during a workplace smoking cessation group training program with incentives 


\section{ABSTRACT}

Objectives Smoking cessation programs in combination with financial incentives have shown to increase quit rates in smokers, but it is not clear which elements of this intervention help smokers to succeed in their quit attempt. The aim of this study was to explore the view of successful and unsuccessful quitters about which factors had affected their ability to quit smoking.

Design Semi-structured qualitative interviews were conducted and analyzed using the Framework method.

Setting Interviews were conducted in 2017 with employees from nine different Dutch companies.

Participants 24 successful and unsuccessful quitters from the intervention group of a cluster randomized controlled trial (RCT) who participated in a workplace smoking cessation group training program in which smoking abstinence was rewarded with financial incentives.

Results Themes that emerged were the workplace setting, quitting with colleagues, motivation, family support, strategies, and the financial incentives. The interviewees reported that the smoking cessation program was appreciated in general, was convenient, lowered the threshold to sign up, stimulated peer support and competition, and provided strategies to resist smoking. Personal motivation and a mind set to never smoke again were regarded as important factors for quit success. The financial incentives were not considered as a main motivator to quit smoking, which contradicts the results from the RCT. The financial incentives were considered as more attractive to smokers with a low income.

Conclusions According to participants, contributors to quitting smoking were the workplace cessation program, personal motivation, and peer support, but not the incentives. More research is needed on the contradiction between the perceived effects of financial incentives on quit success and the actual difference in quit rates. 


\section{INTRODUCTION}

Within companies, promoting smoking cessation is becoming more frequently part of stimulating a healthy lifestyle among employees. The workplace can be a suitable setting for smoking cessation interventions. ${ }^{1}$ It allows reaching smokers in a more direct way than through the healthcare system or mass media campaigns. In addition, attending a smoking cessation intervention facilitated by the employer can be convenient for smokers and relieve financial barriers, ${ }^{2,3}$ while it is simultaneously cost-effective for the employer if it increases productivity. ${ }^{4,5}$ However, smoking cessation at the workplace can be a sensitive topic, and before companies would consider offering a smoking cessation program to their employees, they need to be convinced that this would be appreciated, and not lead to resistance. ${ }^{6}$

Workplace situated smoking cessation group programs have shown to be effective. Led by a health professional, group programs are designed to aid quitting by increasing motivation, improving knowledge and skills, and providing social support. ${ }^{78}$ However, the role of social processes is usually not evaluated in trials. ${ }^{79}$ Co-worker interaction may stimulate or hinder quit success, as may family or friend's influence outside the work environment. ${ }^{3}$ Additionally, individual factors such as motivation to quit ${ }^{10}$, perceived ability to quit, beliefs about smoking ${ }^{3}$ and an identity change towards being a 'nonsmoker'11 may play a role in achieving long term abstinence.

Smoking cessation interventions are sometimes combined with financial incentives to promote attendance or to enhance abstinence rates, which has shown to be effective in various experimental trials. ${ }^{1,12,13}$ Regardless of their effect, smokers do not necessarily consider incentives as a motivation to quit smoking. ${ }^{10,14}$ In a previous study, ${ }^{14}$ incentives were considered as a reward or bonus for quitting, and something "to work towards". Some participants indicated that the reward was a "tipping point" to quitting. It was suggested by service providers that the incentives could encourage participants to stick to their treatment, thereby increasing their chances to quit.

In April 2016, a cluster randomized controlled trial started in the Netherlands that aimed to investigate the effects of a financial incentive on smoking abstinence. ${ }^{15}$ Employees from Dutch companies attended a smoking cessation group training program at the workplace and could earn vouchers for smoking abstinence. The results of this trial showed that after six months, a significantly higher number of participants in the intervention group (training program + incentives) was abstinent compared to the control group (training program only). ${ }^{16}$

Apart from the quantifiable effect of a smoking cessation intervention on quit rates, it is important to investigate how a smoking cessation program with incentives at the workplace is experienced by the employees who actually have participated in such a program, and which factors they consider to be key in achieving quit success. Little research has been done on how employees feel about their employer's initiative to promote smoking cessation, there is a lack of knowledge on the effect of social interaction 
on smoking cessation in a workplace setting, and it is unclear how exactly financial incentives may motivate smokers and lead to higher quit rates.

Therefore, the aim of the current study was to investigate how employees who participated in a workplace group smoking cessation program with financial incentives evaluated this intervention, to assess their perspectives on the incentives, and to identify which success factors had affected their ability to stop smoking.

\section{METHODS}

\section{The randomized controlled trial}

The interviewed participants in the current study were part of a randomized controlled trial (RCT) investigating the effect of incentives on smoking abstinence. ${ }^{15}$ In the RCT, 604 cigarette smoking employees followed a 7-week smoking cessation group training program organized at the workplace by professional stop-smoking coaches experienced in giving smoking cessation group training in a workplace setting. Participants in the intervention group earned gift vouchers with a total value of 350 euros for carbon monoxide (CO) validated continuous smoking abstinence. Participants earned the first voucher of 50 euros if they were abstinent based on self-report, validated by COmeasurement directly after finishing the smoking cessation training. The next 50-euro vouchers were earned if participants were abstinent three and six months after the cessation training, and the final 200 euro voucher was received for smoking abstinence 12 months after finishing the training. The vouchers could be exchanged in a digital gift shop for a broad range of products and activities. The interviews and the qualitative analyses were performed before the results on abstinence rates from the trial were deblinded. Neither the participants, nor the researchers were aware of the outcomes of the RCT during the interviews.

\section{Participants and setting}

Between June and November 2017 we invited 26 Dutch employees from nine companies who were participating in the $\mathrm{RCT}^{15}$ for an interview. Only participants from companies that were within 30 days of the 6-month follow-up moment and from companies that were randomly selected to receive vouchers for quit success were invited for the interviews. Participants were purposefully sampled to obtain an oversampling of successful quitters, in accordance with the aim of investigating the factors contributing to quit success. More participants with a lower income were invited, because the relatively high proportion of smokers in this group makes it an important target group for smoking cessation interventions. Participants first received an email to inform them about the purpose of the interviews and were later called by telephone to ask whether they were interested in participating and to make arrangements. Two persons refused to be interviewed with no specific reason. The interviewers and interviewees had not met before.

\section{Interviews}

A qualitative approach was chosen for this study, in order to investigate participants' experiences and perspectives. Based on a pilot-tested interview guide (main questions 
shown in Table 1), semi-structured interviews were conducted with participants individually. Interviews were conducted by FAB and GEN. FAB was trained in interviewing by GEN, who is a trained and experienced interviewer. In order to match the interview style, for the first two interviews both interviewers were present. Interviews were conducted in a quiet and private space at the workplace or at the participant's home. Participants were informed about the confidentiality of the interview. All participants gave their written informed consent for participating in the study. The interviews explored the participant's experiences with the smoking cessation training program and with their quit attempt. Because the influence of incentives on smoking cessation was of special interest to this research, participants were asked at the end of the interview what they thought about the incentives, and if the topic did not come up spontaneously, whether the incentives had influenced their enrolment in the program or their quit success. Interviews lasted between 20 and 40 minutes each. All interviews were conducted in Dutch and audio recorded.

\section{Analysis}

The audio recordings of the interviews were transcribed verbatim. Interviews were uploaded and analyzed in NVivo 11 according to the "Framework" method. ${ }^{17}$ This is an analytic approach developed for conducting applied qualitative research, and involves a number of stages through which data are analyzed. The stages are: familiarization with the data; creating a thematic framework; indexing; charting; and mapping and interpretation. The data were analyzed by two researchers (FAB and LD). A mixed approach was used of coding based on predefined themes from the topic list, and "open" coding, in which codes were ascribed to data based on their emergence upon reading the transcript. Two researchers (FAB and LD) each coded three assigned transcripts individually, after which the codes were discussed, and a set of codes was agreed upon. Thereafter, based on the set of codes, three more transcripts were analyzed by the two researchers individually, and results were compared for a second time. The code set was adapted after deliberation and used to index the remainder of the interviews. Any newly emerging codes were discussed between the researchers. Codes were combined and rearranged into themes and sub-themes that arose from the data. Based on the topic list, the theme 'financial incentives' was divided into influence of vouchers on enrolment, influence of vouchers on motivation to quit, and appraisal of vouchers. Furthermore, the data were rearranged into success factors for quitting smoking. Additional themes that arose from the data were reasons to quit smoking and barriers to quitting smoking, but these themes were not further described in the current article; instead we chose to focus on the themes that were considered most relevant, which were financial incentives and success factors. Comparing the responses of successful quitters with responses of unsuccessful quitters on these themes revealed no different patterns between these groups. Therefore, the results for the themes are presented for all respondents together. Framework matrices were constructed for the themes financial incentives and success factors for quitting smoking, in which the relevant data for each respondent were integrated. By reviewing the framework matrices and by checking the original transcripts, the data was synthesized and interpreted. Data saturation was reached for the theme incentives after 5 interviews and for the theme success factors after 18 interviews (Figure 1). 


\section{RESULTS}

\section{Participant characteristics}

Table 2 presents summary characteristic of the interviewees. Seventeen men and seven women were interviewed. Half of the participants had a low income. Fifteen participants had been abstinent since the end of the smoking cessation training program, i.e. at least six months. Nine participants had initially quit smoking but relapsed somewhere within the first six months. The participants' age was between 25 and 61 years old. The majority of participants had attempted to quit smoking in the past.

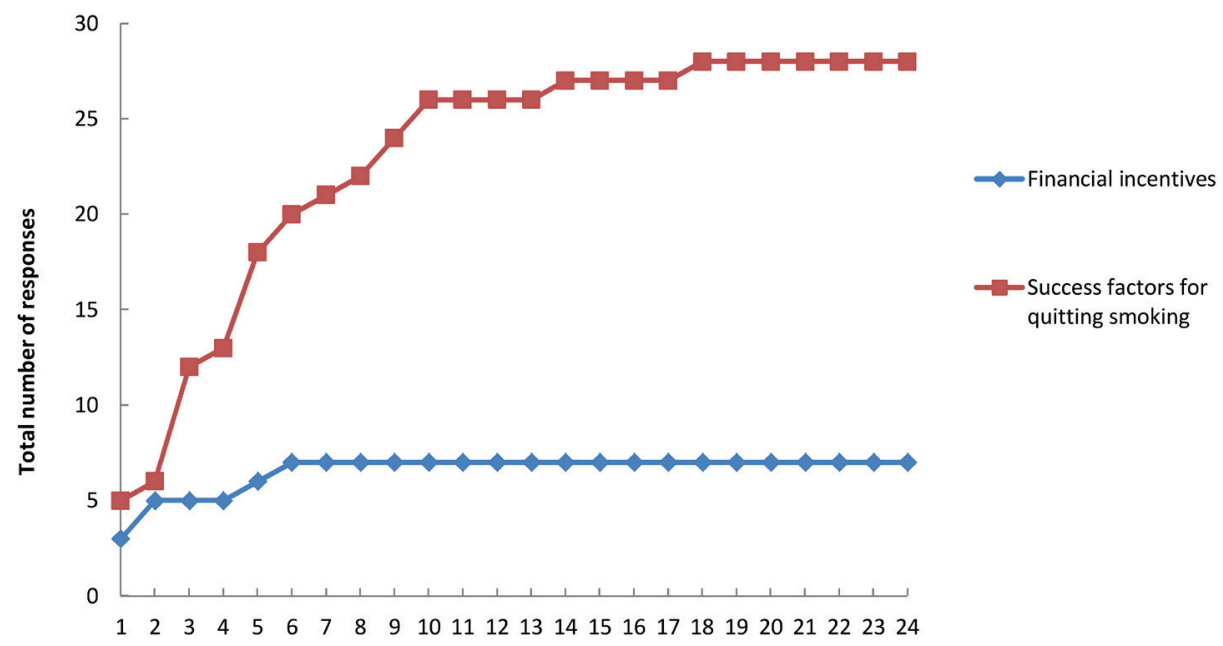

Figure 1. Total number of codes mentioned by the participants for the themes financial incentives and success factors for quitting smoking. 
Table 1. Interview guide

\begin{tabular}{|c|c|}
\hline Topic & Question \\
\hline Opening question & What kind of work do you do? \\
\hline Previous quit attempts & $\begin{array}{l}\text { Have you ever tried to quit smoking before the } \\
\text { smoking cessation training program? }\end{array}$ \\
\hline Choice to participate in the group training program & $\begin{array}{l}\text { How did you learn about the smoking cessation } \\
\text { training program? }\end{array}$ \\
\hline Experiences with the group training program & $\begin{array}{l}\text { If you look back at the smoking cessation training } \\
\text { program, how did you experience it? }\end{array}$ \\
\hline Quitting smoking in the working environment & $\begin{array}{l}\text { What do you think about the fact that the training } \\
\text { program was organized at the workplace? }\end{array}$ \\
\hline Quitting smoking successful or not & How did your attempt to quit smoking go? \\
\hline Success factors in quitting & What helped you to stay quit? \\
\hline The stimulating effect of the reward & What did you think about the reward? \\
\hline Final question & $\begin{array}{l}\text { Would you like to add something to what we have } \\
\text { discussed? }\end{array}$ \\
\hline
\end{tabular}


Table 2. Participant characteristics

\begin{tabular}{|c|c|c|c|}
\hline \multicolumn{2}{|l|}{ Characteristics } & \multicolumn{2}{|c|}{$\begin{array}{c}\text { Number from interviewed } \\
\text { sample }(n=24)\end{array}$} \\
\hline Gender & $\begin{array}{l}\text { Men } \\
\text { Women }\end{array}$ & $\begin{array}{c}17 \\
7\end{array}$ & $\begin{array}{l}70.8 \% \\
29.2 \%\end{array}$ \\
\hline Age group years & $\begin{array}{l}18-25 \\
26-45 \\
46-65 \\
>65\end{array}$ & $\begin{array}{c}1 \\
13 \\
10 \\
0\end{array}$ & $\begin{array}{c}4.2 \% \\
54.2 \% \\
41.7 \% \\
0.0 \%\end{array}$ \\
\hline $\begin{array}{l}\text { Attempted to quit in } \\
\text { the past }\end{array}$ & $\begin{array}{l}\text { Yes } \\
\text { No }\end{array}$ & $\begin{array}{c}19 \\
5\end{array}$ & $\begin{array}{l}79.2 \% \\
20.8 \%\end{array}$ \\
\hline Smoking status & $\begin{array}{l}\text { Current smoker } \\
\text { Abstinent }\end{array}$ & $\begin{array}{c}9 \\
15\end{array}$ & $\begin{array}{l}37.5 \% \\
62.5 \%\end{array}$ \\
\hline $\begin{array}{l}\text { Duration of quit } \\
\text { attempt }\end{array}$ & $\begin{array}{l}<1 \text { week } \\
1-4 \text { weeks } \\
5-12 \text { weeks } \\
13-26 \text { weeks } \\
\text { Abstinent up to } 26 \text { weeks }\end{array}$ & $\begin{array}{c}1 \\
6 \\
2 \\
0 \\
15\end{array}$ & $\begin{array}{c}4.2 \% \\
25.0 \% \\
8.3 \% \\
0.0 \% \\
62.5 \%\end{array}$ \\
\hline Education & $\begin{array}{l}\text { Low } \\
\text { Moderate } \\
\text { High }\end{array}$ & $\begin{array}{c}4 \\
17 \\
3\end{array}$ & $\begin{array}{l}16.7 \% \\
70.8 \% \\
12.5 \%\end{array}$ \\
\hline Income & $\begin{array}{l}\text { Low } \\
\text { Moderate } \\
\text { High } \\
\text { Missing }\end{array}$ & $\begin{array}{l}12 \\
7 \\
3 \\
2\end{array}$ & $\begin{array}{l}50.0 \% \\
29.2 \% \\
12.5 \% \\
8.3 \%\end{array}$ \\
\hline Occupation* & $\begin{array}{l}1 \text { Managers } \\
2 \text { Professionals } \\
3 \text { Technicians and associate professionals } \\
4 \text { Clerical support workers } \\
5 \text { Service and sales workers } \\
6 \text { Skilled agricultural. forestry and fishery workers } \\
7 \text { Craft and related trades workers } \\
8 \text { Plant and machine operators. and assemblers } \\
9 \text { Elementary occupations } \\
0 \text { Armed forces occupations }\end{array}$ & $\begin{array}{l}2 \\
3 \\
4 \\
4 \\
3 \\
1 \\
2 \\
2 \\
3 \\
0\end{array}$ & $\begin{array}{c}8.3 \% \\
12.5 \% \\
16.7 \% \\
16.7 \% \\
12.5 \% \\
4.2 \% \\
8.3 \% \\
8.3 \% \\
12.5 \% \\
0.0 \%\end{array}$ \\
\hline $\begin{array}{l}\text { Number of training } \\
\text { sessions attended } \\
\text { out of } 7\end{array}$ & $\begin{array}{l}1-2 \\
3-4 \\
5-6 \\
7\end{array}$ & $\begin{array}{c}0 \\
2 \\
14 \\
8\end{array}$ & $\begin{array}{c}0.0 \% \\
8.3 \% \\
58.3 \% \\
33.3 \%\end{array}$ \\
\hline $\begin{array}{l}\text { Could attend } \\
\text { majority of training } \\
\text { session within } \\
\text { working hours }\end{array}$ & $\begin{array}{l}\text { Yes } \\
\text { No } \\
\text { Missing }\end{array}$ & $\begin{array}{c}6 \\
16 \\
2\end{array}$ & $\begin{array}{c}25.0 \% \\
66.7 \% \\
8.3 \%\end{array}$ \\
\hline
\end{tabular}

* International Standard Classification of Occupations 2008 (ISCO 2008)

Education categories: low: none completed, primary school and lower secondary education; moderate: middle secondary education; high: upper secondary education and university.

Income categories: individualized net monthly household income,$^{18}$ divided into three categories based on tertiles. 


\section{Financial incentives}

In the interview, participants were asked what they thought about the vouchers, and whether the vouchers had influenced them to enroll in the smoking cessation training program or to quit smoking.

\section{Influence of vouchers on enrolment}

Before enrolling in the smoking cessation training program, participants knew that there was a chance to be eligible to earn vouchers for quit success, but they did not know that they were randomized into the incentives condition until the first session of the training program. The majority of participants said that the gift vouchers had not had any effect on their motivation to enroll, while some thought it added somewhat to their own motivation. Almost all subjects stated that if they would not have been able to earn the reward, they would also have applied for the training program. 'It was not the motive for me. Even if it had been without a reward, I would have done it.' Participant 9, successful quitter, moderate income. The few participants who did consider the gift voucher as a stimulator for enrolling in the training program fell in the low income category. 'So, the intervention group. Sure, that was really something nice to experience. But I would absolutely have taken part anyway, even without it. But yeah - it was definitely an extra incentive.' Participant 2, smoker, low income.

\section{Influence of vouchers on motivation to quit}

None of the participants spontaneously brought up the vouchers as a reason to quit smoking. When asked whether the vouchers were a motivation to quit smoking, most participants stated that they experienced the vouchers as an added bonus, but not as a reason for quitting. 'For me, that wasn't the motivation to do it. It was more like a nice bonus.' Participant 8, successful quitter, income unknown. Participants found it nice to receive the voucher, and it felt good to have earned one, but they quit because they were intrinsically motivated, and had more important reasons to quit smoking than the vouchers, such as their health their children. Some participants had the feeling that it would not be possible to quit smoking just to earn a voucher; inner motivation is crucial if you want to succeed in quitting. 'Being offered a gift certificate wasn't a matter of, 'Oh gee, I better quit because, you know, I can earn money if I do.' No, that's pointless, because you won't be successful that way. You definitely won't go the distance like that.' Participant 5, successful quitter, low income. It was mentioned that the urge to smoke was stronger than even a much larger hypothetical reward. One subject thought that the voucher could subconsciously have contributed to quit success. 'Subconsciously, it will indeed be a stimulus that makes you think: Oh, that's a nice added benefit.' Participant 3, successful quitter, low income. Another participant thought that the voucher had been a motivator to stay abstinent from smoking. 'Yes, it did factor in. I think that it did actually serve as a motivation to keep going.' Participant 2, smoker, low income.

\section{Appraisal of the vouchers}

Participants were asked how they felt about the incentive. Most participants liked and appreciated the vouchers as a reward for quit success. 'I like it; I've been using it to do fun things, too.' Participant 17, successful quitter, moderate income. There were also a few subjects who had not used the vouchers yet. Some participants had not paid much 
attention towards the gift. 'Well, to be honest: I haven't done anything with it yet. I haven't given it a bit of thought, either.' Participant 12, smoker, high income. It is notable that the participants who had not yet spent the vouchers had a relatively high income. Although most participants considered the amount of the vouchers generous, some participants found that the vouchers were not large enough to motivate them because they saved a lot more money by not buying cigarettes anymore compared to the amount of the vouchers. 'I mean, most people think the same way that I do: you earn more by quitting on your own than with that reward. If you smoke a pack a day at around $€ 6.50$, you have already earned more per week than the gift voucher is worth.' Participant 6, smoker, low income.

\section{Success factors for quitting smoking}

The following paragraphs describe the factors that according to the participants had positively affected their ability to stop smoking.

\section{Smoking cessation at the workplace}

Participants found it encouraging and saw it as an opportunity that the employer offered a smoking cessation training program. Some participants mentioned that their employer's involvement was an additional motivator to quit smoking. 'It motivates as well if management asks: oh, did you manage to quit? You know, that's also a motivation and nice to hear.' Participant 7, successful quitter, low income. Several participants mentioned that by offering the smoking cessation program, employers showed their care for the employees' health and wellbeing. The majority of participants found it easy and convenient that the training program was situated at the workplace. Participants reported that the threshold to sign up was low because the workplace was a familiar and safe environment, because they did not have to travel to attend meetings, and because it did not cost them extra time. 'Somehow, you sign up sooner than if it were outside work.' Participant 15, smoker, high income. Only a couple of participants said that the location of the smoking cessation training program was all the same to them and that they would have attended the training program as well when it was held at a different location.

\section{Quitting smoking with colleagues}

The majority of participants enjoyed the social aspect of quitting smoking in a group. They felt supported and encouraged by their fellow quitters and enjoyed sharing experiences during the training sessions. It felt motivating to quit with others to have a mutual goal. 'It's good that such a group gives you confirmation that you're on the right track, but also that you can reach out to each other. Yes, you're going through the same things, basically.' Participant 11, successful quitter, moderate income. Many participants described a feeling of peer pressure and competition among colleagues, which motivated them to refrain from smoking. 'Secretly, it's a competition, of course; I mean, you're looking around, going: "Right, who's the weakling that's going to cave in first." Everyone placed their bet, obviously. It wasn't me, thank goodness, but still. No one wants to be a loser, it seems to me.' Participant 10, smoker, high income. Attending the training program with colleagues compared to strangers was an added value to some participants. 'Yeah, you almost spend more time with them, on a daily basis, than with your own family. So, yeah, I guess what that means is, that when you quit at work, you see those people for a big part of every day and then there's the check too and wanting to prove to them, 'See, that's right, I'm still off smoking.' That's quite 
nice.' Participant 22, smoker, moderate income. Other participants stated that it did not matter to them who the other group members were. For some participants, an additional advantage of following the training program with colleagues was that they could go to them during the workday for support when they were craving for a cigarette. 'Then at $a$ certain point, you start to crave a cigarette again, so you walk over there (laughs) and you're like: 'How're things going with you, then?' Participant 6, smoker, low income.

\section{Motivation}

Many participants mentioned they had succeeded in quitting smoking because they continued to be very motivated along the quit attempt. They said that without enough motivation and willpower it would not be possible to quit smoking. 'First of all, you have to be completely ready for it, you have to be open to it, you have to really want it fully and the motivation has to be there. And also, that you can do it and that everyone can do it. Even if you're a heavy smoker, everyone can do it.' Participant 14, successful quitter, low income. Participants constantly reminded themselves that they were no longer smokers, and that they would never smoke again. 'You've quit. You have to flip a switch, because if you don't flip that switch yourself by saying: "I've quit or l absolutely want to quit," well, it's not going to work.' Participant 5, successful quitter, low income. The decision not to smoke again seemed a very important contributor to quit success and was reported by almost half of the successful quitters.

\section{Family support}

The support from close family members such as spouse or children was an important factor to stay motivated during the quit attempt for many participants. Some participants said that their family members did not smoke and disapproved of smoking, which motivated them to quit and persevere in their attempt. 'My kids really hate smoking. Nobody else around them smokes. So yeah, they think it's dirty, they think it stinks, they think I smell of the smoke. Those are things that really helped keep me motivated to quit.' Participant 1, successful quitter, high income. Participants also reported mental support and positive affirmation from their families, who expressed happiness and pride for the participant's quit success. Some participants mentioned that their spouse had simultaneously decided to quit smoking, and that quitting together facilitated mutual support during cravings at home. 'It was nice to quit together with someone else. That way, there's two of you, both in the same boat - and both of you are nice and cranky (laughs). But it does help. It means you get that support from one another, even when you're at home.' Participant 21, successful quitter, high income. Some of these participants believed that they would not have succeeded in their quit attempt if their spouse had not quit smoking as well.

\section{Strategies}

An additional important success factor was the strategies to cope with craving and methods to stay quit that participants had learned during the smoking cessation training program. Almost half of the subjects gave an example of a strategy they used at difficult moments, for example the knowledge that craving only lasts a few minutes and that they should distract themselves at those moments. 'Those are the moments you have to make a change by doing something else instead: going for a walk, maybe, or grabbing a cup of coffee. I got that tip from [name of trainer] actually, and it's made things a lot easier.' Participant 13, 
smoker, moderate income. The strategies not only helped as a practical advice on how to act in difficult situations, but also provided reassurance and gave participants the confidence that they would be able to cope with the situation. 'It got me thinking. When I have a craving for a cigarette, for example, I think: oh, it'll only be three minutes and then it will have passed. Well, that's good. For me, that's a reassuring thought: Okay, it will be over quickly. And then I can go back to doing whatever I'm doing.' Participant 12, smoker, low income.

\section{DISCUSSION}

By interviewing employees who had participated in a workplace smoking cessation training program in which they could earn vouchers for quit success, this study aimed to investigate what successful and unsuccessful quitters considered to have contributed to their quit success. In particular, it was assessed whether incentives had motivated participants to quit smoking.

The vouchers for quit success were appreciated by the participants, but most participants said that the vouchers had not been a reason for them to sign up for the smoking cessation group training and had not been a motivation to quit smoking. They indicated that they had quit smoking because they were very motivated to quit, that you should not quit for a reward, and if you did, you probably would not be successful. Interestingly, the interviewed participants were part of a large cluster randomized trial that showed significantly higher abstinence rates in the incentive group compared to the control group. ${ }^{16}$ Both the interviewed participants and the interviewers were unaware of this result during the interviews. How can it be explained that although participants in the interviews claimed that they were not motivated by the vouchers, the trial results do show a substantial effect of the vouchers? Because in the trial the intervention and control group received the same smoking cessation group training, the difference in quit rates between the groups cannot be attributed to the group training. In previous research with pregnant women,${ }^{19}$ it was suggested that there is a possibility that participants considered it socially unacceptable to quit smoking for money and were therefore hesitant to admit (perhaps even to themselves) that the vouchers were a motivating factor. In the current study, both interviewers perceived that the interviewees were very open and honest about their experiences. As a result, the participants' responses should be valued as truthful, which leaves open the possibility that participants may have been influenced by the vouchers subconsciously. This idea is supported by research in the field of behavioral sciences and decision-making, showing that people may not always have insight in what guides their behavior. ${ }^{20,21} \mathrm{~A}$ possible explanation based on the interviews in the current study, is that participants did not recognize an additional effect of the vouchers because they were intrinsically motivated to quit smoking to such a large degree and found the group training so helpful, that this overshadowed the smaller additional motivational value of the vouchers. Another hypothesis posed in a previous study is that smokers who successfully quit and are satisfied with their achievement, in retrospect possibly discount the importance of the incentives on their quit attempt. ${ }^{10}$ This self-serving bias, ${ }^{22}$ where individuals tend to attribute their successes to internal factors but failure to external causes, may explain why the vouchers were not recognized as a contributor to quit 
success. Considering these potential subconscious influences, future studies may use a combination of different data collection methods and employ interview techniques that stimulate introspection. For example, to prevent social desirable answers, participants could be asked whether they think vouchers would be motivating for other smokers. ${ }^{23}$ Likewise, participants could be asked about hypothetical incentives, which was done in a qualitative study where employees endorsed incentives as potentially effective because they were "motivating, affirming and challenging". ${ }^{6}$

An interesting finding was that the few participants who mentioned that the incentives were motivating had a lower income. It is likely that financial incentives are valued more by smokers who have less money to spend. Although previous research has not yet found a decisive answer on this hypothesis, a systematic review found evidence that financial incentives may be more effective in influencing health behavior in participants with a higher deprivation level. ${ }^{24}$

The current study showed that a workplace situated smoking cessation training program is convenient to smokers because they do not have to spend extra time to travel to a different location and the workplace is a familiar setting. In line with this result, in a previous study participants named lack of time and inconvenience as reasons for not participating in a smoking cessation programme. ${ }^{10}$ It is also conceivable that participants are more likely to sign up for a smoking cessation program when it is "brought to them" via their work, instead of having to search for counselling options themselves. This way, smokers could be reached that would not actively search for smoking cessation counselling. The participants appreciated that the employer covered the cost of the training. Previous research has also found that covering the cost of smoking cessation therapy increases its use. ${ }^{25}$ Finally, the interviewed employees appreciated the employers' initiative to offer a smoking cessation training program, because it was seen as an expression of caring for the employees' health. Employees viewed the training program as an opportunity and considered it an appropriate addition to the current company health plan. This is an important result for employers, since they could be hesitant to offer a smoking cessation training if they think that it would mean intervening too much in employees' personal health decisions. ${ }^{6}$ In order to maximize participation, it may therefore be important to think about how a smoking cessation training program is framed and communicated. ${ }^{26}$ This was confirmed by a study in which participants said that employers should avoid being "pushy" and should avoid activities "shoved down [their] throats". ${ }^{\text {The employers from }}$ the current study presented the training as an opportunity for employees who wanted to quit smoking, and enrolment was on a voluntary basis without imposed pressure. This has most likely contributed to the positive appraisal of the employer offering the smoking cessation program by the interviewees.

The interviews also show that the social aspect of participating in a group training with colleagues was an important success factor. Participants felt supported by their colleagues both within counselling sessions and during the workday, and the social pressure of not wanting to be the first person to relapse was a motivator to stay abstinent. The group setting stimulated competition between the participants; friendly competition was also considered an important motivating element that should be incorporated in smoking 
cessation interventions according to a qualitative study involving current smokers. ${ }^{27}$ In previous research it was suggested that group training participants develop a "team-stop smoker" identity where quitting smoking was considered as a collective effort. ${ }^{11}$ These results indicate that group dynamics are an meaningful contributor to quit success. It should be further investigated which factors influence the effectiveness of the group process, and how social networks of colleagues at the workplace influence quit success. For example, in previous research it was found that in smaller companies where employees knew one another, the likelihood that a participant stopped smoking increased by $34 \%$ if a co-worker quit smoking. ${ }^{28}$ In the current study, there were mixed reactions on type of fellow quitters; some participants said they felt more supported by close colleagues while others said that it did not matter to them whether they knew the other group members or not. Additionally, it is possible that other group members' success or failure in quitting smoking influences the success of the entire group. It was suggested in another study that a"rolling" group where new people can join may have the benefit of including experienced members who went through the quitting process and can share their advice. ${ }^{14}$

\section{Strengths and limitations}

An important strength of this study is the use of a qualitative methodology which enabled us to assess in-depth the personal experiences of participants. Furthermore, this study had a high response and reached saturation on the main topics. A limitation of this methodology is that the small number of participants may represent a particular selection of the general population and results may not be generalizable. For example, employees disapproving of a workplace smoking cessation group training program probably did not subscribe. However, generalizability is not the purpose of qualitative research, and this approach provided the possibility to investigate successful and unsuccessful quitters' view on the incentives and on success factors in the process of quitting smoking. Finally, incentives for quitting smoking may be a sensitive topic and could be prone to socially desirable answers.

\section{Conclusion}

This study showed that according to participants, a workplace smoking cessation group training program with incentives provided several facilitators that helped them quit smoking. As stated by the interviewees, the workplace environment was convenient and facilitated peer support. The training program provided helpful strategies which participants could employ to resist craving. From the participants' testimonies, it was also apparent that personal motivation to quit smoking and a mind set to never smoke again were important promoting factors for quit success. The vouchers were appreciated as an additional factor and were not a main reason to quit smoking according to the participants. The effect of vouchers on abstinence found in the RCT could be explained by a subconscious stimulating effect. The findings of this study are an important contribution to understanding which specific factors according to employees who participated in a workplace group smoking cessation training program combined with incentives have influenced their quit success and can be used to improve existing smoking cessation interventions. More research is needed to explain the contradictory effect of incentives on quit success which is found if opinions of successful and unsuccessful quitters are compared with actual quit rates. 


\section{REFERENCES}

1. Cahill K, Lancaster T. Workplace interventions for smoking cessation. The Cochrane Library. 2014.

2. Smith AL, Carter SM, Chapman S, Dunlop SM, Freeman B. Why do smokers try to quit without medication or counselling? A qualitative study with ex-smokers. BMJ Open. 2015;5(4):e007301.

3. Carroll C, Rick J, Leaviss J, Fishwick D, Booth A. A qualitative evidence synthesis of employees' views of workplace smoking reduction or cessation interventions. BMC Public Health. 2013;13:1095.

4. Berman M, Crane R, Seiber E, Munur M. Estimating the cost of a smoking employee. Tob Control. 2014;23.

5. Sherriff NS, Coleman L. Understanding the needs of smokers who work as routine and manual workers on building sites: results from a qualitative study on workplace smoking cessation. Public Health. 2013;127(2):125-133.

6. Tiede LP, Hennrikus DJ, Cohen BB, Hilgers DL, Madsen R, Lando HA. Feasibility of Promoting Smoking Cessation in Small Worksites: An Exploratory Study. Nicotine \& Tobacco Research. 2007;9(Suppl_1):S83-S90.

7. Stead LF, Carroll AJ, Lancaster T. Group behaviour therapy programmes for smoking cessation. Cochrane Database Syst Rev. 2017(3).

8. Kotsen C, Santorelli ML, Bloom EL, et al. A Narrative Review of Intensive Group Tobacco Treatment: Clinical, Research, and U.S. Policy Recommendations. Nicotine \& Tobacco Research. 2018:nty162-nty162.

9. Dimoff JD, Sayette MA. The case for investigating social context in laboratory studies of smoking. Addiction. 2017;112(3):388-395.

10. Kim A, Kamyab K, Zhu J, Volpp K. Why are Financial Incentives not Effective at Influencing Some Smokers to Quit? Results of a Process Evaluation of a Worksite Trial Assessing the Efficacy of Financial Incentives for Smoking Cessation. J Occup Environ Med. 2011;53(1):62-67.

11. Vangeli $E$, West R. Transition towards a 'non-smoker' identity following smoking cessation: An interpretative phenomenological analysis. Br J Health Psychol. 2012;17(1):171-184.

12. Cahill K, Hartmann-Boyce J, Perera R. Incentives for smoking cessation. Cochrane Database Syst Rev. 2015(5).

13. Giles EL, Robalino S, McColl E, Sniehotta FF, Adams J. The Effectiveness of financial incentives for health behaviour change: systematic review and meta-analysis. PLoS One. 2014;9.

14. Ormston R, van der Pol M, Ludbrook A, McConville S, Amos A. quit4u: the effectiveness of combining behavioural support, pharmacotherapy and financial incentives to support smoking cessation. Health Educ Res. 2015;30(1):121-133.

15. Van den Brand FA, Nagelhout GE, Winkens B, et al. The effect of financial incentives on top of behavioral support on quit rates in tobacco smoking employees: study protocol of a clusterrandomized trial. BMC Public Health. 2016;16(1):1056.

16. Van den Brand FA, Nagelhout GE, Winkens B, Chavannes NH, van Schayck OCP. Effect of a workplace-based group training program combined with financial incentives on smoking cessation: a cluster-randomized controlled trial. The Lancet Public Health. 2018;3(11):e536-e544. doi:10.1016/S2468-2667(18)30185-3.

17. Ritchie J, Spencer L. Qualitative data analysis for applied policy research. The qualitative researcher's companion. 2002;573(2002):305-329.

18. OECD. What are equivalence scales? http://www.oecd.org/eco/growth/OECD-NoteEquivalenceScales.pdf. Updated 15-12-2017. Accessed 15-12-2017, 2017. 
19. Mantzari E, Vogt $F$, Marteau TM. The effectiveness of financial incentives for smoking cessation during pregnancy: is it from being paid or from the extra aid? BMC Pregnancy Childbirth. 2012;12(1):24.

20. Newell BR, Shanks DR. Unconscious influences on decision making: A critical review. Behav Brain Sci. 2014;37(1):1-19.

21. Nisbett RE, Wilson TD. Telling more than we can know: Verbal reports on mental processes. Psychol Rev. 1977;84(3):231.

22. Weiner B. An attributional theory of achievement motivation and emotion. Psychol Rev. 1985;92(4):548-573.

23. Krefting L. Rigor in qualitative research: The assessment of trustworthiness. Am J Occup Ther. 1991;45(3):214-222.

24. Mantzari E, Vogt F, Shemilt I, Wei Y, Higgins JP, Marteau TM. Personal financial incentives for changing habitual health-related behaviors: A systematic review and meta-analysis. Prev Med. 2015;75:75-85.

25. Van den Brand FA, Nagelhout GE, Reda AA, et al. Healthcare financing systems for increasing the use of tobacco dependence treatment. Cochrane Database Syst Rev. 2017(9).

26. Fishwick D, Carroll C, McGregor M, et al. Smoking cessation in the workplace. Occup Med (Lond). 2013;63(8):526-536.

27. Bottorff JL, Oliffe JL, Sarbit G, Sharp P, Kelly MT. Smoke-Free Men: Competing and Connecting to Quit. Am J Health Promot. 2018;32(1):135-142.

28. Christakis NA, Fowler JH. The Collective Dynamics of Smoking in a Large Social Network. NEngl J Med. 2008;358(21):2249-2258. 



\section{CHAPTER 7}
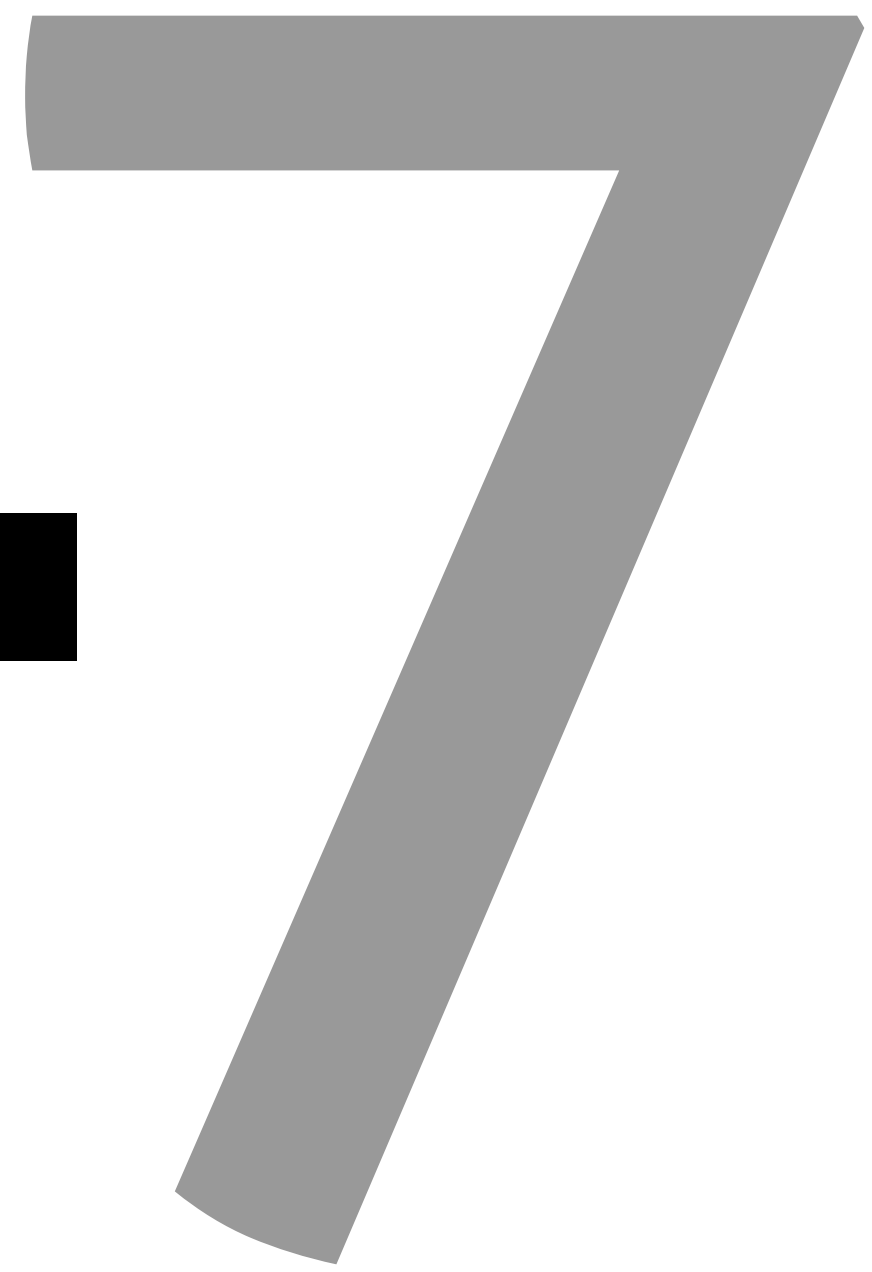


\section{The association of peer smoking behavior and social support with quit success in employees who participated in a smoking cessation intervention at the workplace}

Van den Brand FA, Nagtzaam P, Nagelhout GE, Winkens B, van Schayck CP. The Association of Peer Smoking Behavior and Social Support with Quit Success in Employees Who Participated in a Smoking Cessation Intervention at the Workplace 


\section{ABSTRACT}

The current study investigated whether quit success among employees who participated in a smoking cessation intervention at the workplace was associated with social support from, and the smoking behavior of, people in their environment. Tobacco-smoking employees $(n=604)$ from 61 companies participated in a workplace group smoking cessation program. Participants completed questionnaires assessing social support from, and the smoking behavior of, people in their social environment. They were also tested for biochemically validated continuous abstinence directly after finishing the training and after 12 months. The data were analyzed using mixed-effects logistic regression analyses. Social support from colleagues was positively associated with 12-month quit success (odds ratio $(\mathrm{OR})=1.85,95 \%$ confidence interval $(\mathrm{Cl})=1.14-3.00, p=0.013)$. Support from a partner was positively associated with short-term quit success $(\mathrm{OR}=2.01,95 \% \mathrm{Cl}=$ $1.23-3.30, p=0.006$ ). Having a higher proportion of smokers in the social environment was negatively associated with long-term abstinence $(\mathrm{OR}=0.81,95 \% \mathrm{Cl}=0.71-0.92, p=$ 0.002). Compared to having a non-smoking partner, long-term quit success was negatively associated with having no partner $(\mathrm{OR}=0.48,95 \% \mathrm{Cl}=0.26-0.88, p<0.019)$, with having a partner who smokes $(\mathrm{OR}=0.40,95 \% \mathrm{Cl}=0.24-0.66, p<0.001)$, and with having a partner who used to smoke $(\mathrm{OR}=0.47,95 \% \mathrm{Cl}=0.26-0.86, p=0.014)$. In conclusion, people in a smoker's social environment, particularly colleagues, were strongly associated with quit success. The workplace may, therefore, be a favorable setting for smoking cessation interventions. 


\section{INTRODUCTION}

Current smoking cessation therapy focusses mainly on the individual smoker who wants to quit, although smoking is a social behavior that is greatly influenced by a smoker's social environment. ${ }^{1-3}$ Smoking cessation group therapy, where groups of individual smokers quit together, is designed to stimulate peer support and peer pressure in order to improve the quit success of participants. ${ }^{4}$ However, during most smoking cessation treatments, the wider social environment of the quitter, including family, friends and colleagues, is usually not actively involved. This is unfortunate, since social support from, and the smoking behavior of people in the social environment may be key factors in quit success. ${ }^{2,5,6}$

Longitudinal studies have shown evidence that smokers with a partner who smokes are less likely to attempt to quit and to quit successfully, while having a non-smoking partner can promote quitting. ${ }^{6,7}$ Smokers who live in a household with a larger proportion of smokers are less likely to quit smoking ${ }^{8,9}$ and smokers who have more smokers among their friends are less likely to attempt to quit and to succeed in a quit attempt., ${ }^{2,10}$ The social environment can also promote quitting smoking, for example if smokers perceive that their community has strong anti-smoking norms ${ }^{11}$ or if smokers are motivated to quit by people close to them. ${ }^{12}$ The negative influence of other smokers on quit success may be caused by a lack of social support for quitting smoking, ${ }^{13,14}$ more positive norms toward ${ }^{1}$ and more exposure to smoking cues during a quit attempt. ${ }^{15}$ These results show that it can be more difficult to quit smoking for people who have many smokers in their social environment.

Apart from the home environment, the workplace can be considered as another social setting where people spend a large part of the day together, interact, and may affect each other's beliefs and behaviors. ${ }^{16,17}$ Frequent exposure to others smoking at work was associated with a lower likelihood of recent smoking cessation among workers in a nationally representative sample of US adults. ${ }^{17}$ Another study hypothesized that coworkers may function as models, and found clustering effects of smoking status and the amount smoked in work groups. ${ }^{16} \mathrm{~A}$ network analysis showed that a co-worker who quits smoking increased the likelihood of a subject's smoking cessation by $34 \%$. The influence of co-workers could, therefore, potentially be used in interventions to stimulate smoking cessation in the social setting of the workplace. For instance, group smoking cessation programs with colleagues can have the extra advantage of stimulating social support and peer pressure from people who are nearby for an important part of the day. ${ }^{18}$ However, little research has been done on the potentially valuable effect of social support from colleagues on smoking cessation in a workplace intervention setting. ${ }^{4}$

Although people in the social environment have been shown to influence smoking cessation, it is not entirely clear at what stage of the cessation process social dynamics are particularly influential. Some studies found that positive social support was associated with short-term, but not with long-term quit success, while the absence of negative support such as criticism predicted continuous abstinence. ${ }^{5,19,20}$ Having a partner who also quits smoking at the time of a quit attempt was positively associated with smoking abstinence in older couples up to many years later. ${ }^{6}$ It is important to examine further 
whether support from, and the smoking behavior of the social environment can affect only short-term or also long-term quit success, as this can inform health promotors on when social support can be effective or show when social support should be enhanced.

The current study is a secondary analysis from a cluster randomized trial, in which employees participated together with colleagues in a smoking cessation group training program at their workplace. In the current study, we investigated whether the social influence of different types of people in the environment of the participant, including colleagues who were peer quitters in the group smoking cessation program, was associated with quit success. We also assessed associations of the number of smokers among people in the close circle of the participant (family, friends, and colleagues) and the smoking status of their partner with successful smoking cessation. Additionally, we investigated whether the extent to which these persons were supportive toward the participant's quit attempt was related to quit success. We assessed this social influence on short-term quit success directly after the smoking cessation group training program, and on long-term quit success 12 months later.

\section{METHODS}

\section{Design}

The current study is a secondary analysis of data from a cluster randomized trial (RCT), of which the methods and results have been described in more detail in previous publications..$^{21,22}$ In short, the RCT involved 61 companies organizing a smoking cessation group training at the workplace, which consisted of seven weekly 90-minute sessions divided over a period of two months. In the RCT, 31 companies were randomized into the intervention group and 30 into the control group. In total, 604 employees participated in the RCT. In the intervention group, employees earned vouchers of $€ 350$ in total if they successfully quit smoking for 12 months. The study was registered in the Netherlands Trial Register under number NL5537. The study was approved by the medical ethical committee METC-Z, Heerlen, The Netherlands (No. 16-N-63). All participants signed for their informed consent.

\section{Setting and Participants}

The participants were 604 tobacco-smoking employees aged at least 18 from companies in the Netherlands. Data were collected between March 2016 and March 2018.

\section{Measurements}

To assess social influence, the same questionnaire was sent out at baseline, and at four follow-up measurements: directly after the smoking cessation training had ended (two months after baseline), three months, six months and 12 months after the training had ended. In case of no response, participants were called by phone or were sent a text message with a reminder to fill out the questionnaires. Participants were awarded a voucher of $€ 25$ for completing the questionnaires. Smoking abstinence was assessed directly after the training and at 12 months after the training by a research assistant who 
visited the study participants at their workplace or at home. The research assistant used a Smokerlyzer (Bedfont) to measure expired-air carbon monoxide (CO) concentrations.

\begin{abstract}
Variables
The main outcome variables were continuous abstinence from smoking directly after finishing the smoking cessation program and continuous abstinence 12 months after finishing the program, confirmed by $\mathrm{CO}$ measurement with a threshold value of 9 parts per million. ${ }^{23}$ Participants with measurements above the threshold or participants who we were not able to measure were considered smokers in the analyses. ${ }^{23}$ Among the independent variables was the number of smokers in a participant's social environment, which was measured at baseline with the question: "How many of the five closest friends, acquaintances or colleagues that you regularly spend time with are smokers?" Response categories were $0-5$.
\end{abstract}

We assessed the smoking status of the partner at baseline (T0) and directly after finishing the training (T1), using the question: "Does your partner smoke?". Response categories were: "yes", "no", "I do not have a partner" or "don't know". A new variable was created by combining the smoking status of the partner at baseline and at the end of the cessation program. This resulted in the categories "never smoker","continued smoking", and "stopped between T0 and T1". Participants who answered "I do not have a partner" at both T0 and T1 were categorized as "no partner". All other combinations of answers were categorized as "other". “Don't know" was categorized as missing.

Other independent variables were the social support that participants received from colleagues who also participated in the group smoking cessation program, colleagues who did not participate in the cessation program, their partner, and friends and family. These were assessed with the question "How supportive were (colleagues who participated in the training program/other colleagues/partner/friends and family) of your quit attempt in the last two months?" at follow-up directly after the smoking cessation program. Response categories were "don't know", "very supportive", "moderately supportive", and "a little supportive". For the analyses, the "very supportive" category was retained. The last two response categories were combined into "not very supportive", and the "don't know" category was classified as missing. For the variable "social support from partner", the response category "I do not have a partner" was combined with "not very supportive" in the multivariable analysis to prevent collinearity effects with the "I do not have a partner" response option in the questions assessing the smoking status of the partner.

Additional covariates in the multivariable analysis were measured at baseline and included intervention group (incentive vs. no incentive), nicotine dependence (Fagerström score $0-10){ }^{24}$ income level, and educational level. Income level consisted of individualized net household income and was based on tertiles. Educational level was 'low' for none completed, elementary school and lower secondary education; 'moderate' for middle secondary education; and 'high' for upper secondary education and university. 


\section{Statistical Analyses}

Differences between participants lost to follow-up and participants included in the analyses were tested using independent $T$ tests for numerical variables and Chi square tests for categorical variables. To assess the influence of the social environment on smoking abstinence, we performed separate analyses for each individual variable, correcting only for the covariates (intervention group, income level, educational level, and nicotine dependence). For the outcome "smoking abstinence after 12 months", we included the same covariates and we additionally selected the variables with a $P$ value lower than 0.200 and included these in a multivariable analysis, because the expected that a small number of quitters did not allow including all variables and covariates in the model. We used multivariable mixed-effects logistic regression analysis with a random intercept at company level to adjust for the clustering of participants within a company. Participants with missing data on the outcome "smoking abstinence" were considered as smokers and included in the intention to treat analyses. Other missing data were imputed using all other fixed variables that were included in the model, and values on these variables from other measuring points. We created 50 complete datasets using multiple imputation with a maximum number of 20 iterations and used trace lines to check convergence. We performed a complete case analysis as a sensitivity analysis that only included participants without any missing values. We considered two-sided $p$ values $\leq 0.05$ as statistically significant. IBM SPSS statistics for Windows (version 25.0) (Arrmonk, New York, USA) was used to compute descriptive statistics, RStudio version 1.1.383 MICE (Boston, MA, USA) package for multiple imputation, and Ime4 package (glmer function) for the mixed-effects logistic regression analysis.

\section{RESULTS}

\section{Participants}

The mean age of the participants was 45.1 (standard deviation (SD) 10.2) and there were more male than female participants (Table 1). The mean Fagerström score was 4.4 (SD 2.0), which indicates a moderate nicotine dependence. ${ }^{25}$ Of the 469 participants who had a partner at baseline, $43 \%$ had a partner who smoked (result not reported in Table 1). The mean number of smokers within the participants' close circle of five friends, family or colleagues at baseline was 2.8 (SD 1.5).

\section{Loss to Follow-Up}

There were no missing data on the outcome "smoking cessation". Compared to participants who completed the follow-up questionnaire directly after finishing the smoking cessation training $(n=542)$, participants who did not complete this questionnaire $(n=62)$ more often had a low income and a higher nicotine dependence, but did not differ significantly in terms of educational level, age, sex, or intervention group. 
Table 1. Baseline characteristics of participants.

\begin{tabular}{lc}
\hline Characteristic & Participants \\
\hline Age $(\boldsymbol{n}=\mathbf{5 9 9})$ & \\
Mean (standard deviation (SD)) & $45.1(10.2)$ \\
Sex ( $\boldsymbol{n}=\mathbf{6 0 4})$ & \\
Women & $381(37 \%)$ \\
Men & $223(63 \%)$ \\
Educational level ( $\boldsymbol{n}=\mathbf{5 7 9 )}$ & \\
Low & $159(27 \%)$ \\
Moderate & $255(44 \%)$ \\
High & $165(29 \%)$ \\
Income level ( $\boldsymbol{n}=\mathbf{5 3 5})$ & \\
Low & $179(33 \%)$ \\
Moderate & $175(33 \%)$ \\
High & $181(34 \%)$ \\
Nicotine dependence (Fagerström score 0-10) (n=573) & \\
Mean (SD) & $4.4(2.0)$ \\
Minimally dependent (<4) & $184(32.1 \%)$ \\
Moderately dependent (4-6) & $305(53.2 \%)$ \\
Highly dependent (7-10) & $84(14.7 \%)$ \\
Number of smokers within five friends, acquaintances or colleagues $(\boldsymbol{n}=\mathbf{5 7 9})$ & \\
Mean (SD) & \\
0 & \\
1 & $3.8(1.5)$ \\
2 & $42(7 \%)$ \\
3 & $76(12 \%)$ \\
4 & $130(22 \%)$ \\
5 & $124(21 \%)$ \\
\hline
\end{tabular}

Data are $\mathrm{n}(\%)$ or mean (SD).

\section{Short-Term and Long-Term Abstinence of Participants}

Table 2 presents the CO-validated continuous smoking abstinence of participants. Directly after completing the smoking cessation program, 482 of 604 participants (80\%) had successfully quit smoking. At 12 months follow-up, 206 of 603 participants (34\%) abstained from smoking (one participant was excluded from the 12-month analysis because of unavoidable loss to follow-up according to the Russell Standard). 
Table 2. Participant smoking status, partner smoking status, and social support during the period of the smoking cessation program.

\begin{tabular}{lc}
\hline Characteristic & Participants \\
\hline Participant smoking status ${ }^{\text {A }}$ & \\
Abstinent after smoking cessation program completion $(n=604)$ & \\
Yes & $482(80 \%)$ \\
No & $122(20 \%)$ \\
Abstinent 12 months after smoking cessation program completion $(n=603)^{\text {B }}$ & \\
Yes & $206(34 \%)$ \\
No & $397(66 \%)$ \\
Partner smoking status ( $\boldsymbol{n}=\mathbf{5 0 8})$ & \\
Never smoked & $237(47 \%)$ \\
Continued smoking & $108(21 \%)$ \\
Stopped & $63(12 \%)$ \\
No partner & $96(19 \%)$ \\
Other & $4(1 \%)$ \\
Group training colleague support ( $\boldsymbol{n}=\mathbf{5 0 3})$ & $112(22 \%)$ \\
Not very supportive & $391(78 \%)$ \\
Very supportive & \\
Other colleague support $(\boldsymbol{n}=\mathbf{5 0 0})$ & $304(61 \%)$ \\
Not very supportive & $196(39 \%)$ \\
Very supportive & \\
Friends and family support $(\boldsymbol{n}=\mathbf{5 0 9})$ & $247(49 \%)$ \\
Not very supportive & $262(51 \%)$ \\
Very supportive & \\
Partner support $(\boldsymbol{n}=\mathbf{5 1 6})$ & $133(26 \%)$ \\
Not very supportive & $106(20 \%)$ \\
No partner & $277(54 \%)$ \\
\hline Very supportive &
\end{tabular}

Data are $\mathrm{n}(\%)$ or mean (SD); ${ }^{A}$ CO-validated continuous smoking abstinence; ${ }^{\mathrm{B}}$ One participant was excluded from the analysis because of unavoidable loss to follow-up according to the Russell Standard.

\section{Associations of Social Support with Quit Success}

Table 2 presents how supportive the social environment was during the period from the start until right after finishing the smoking cessation program. A large majority $(78 \%)$ of the participants found their colleagues who also participated in the smoking cessation group training very supportive. By contrast, only $39 \%$ of the participants reported other colleagues to be very supportive. The support of friends and family was approximately equally divided between not very supportive and very supportive. Of the participants, $20 \%$ did not have a partner. Among the participants who did report having a partner, $68 \%$ (277 out of 410) found their partner very supportive of their quit attempt. Table 3 shows that having very supportive group training colleagues was significantly and positively associated with quit success directly after finishing the smoking cessation program (odds ratio $(\mathrm{OR})=3.63,95 \%$ confidence interval $(\mathrm{Cl})=2.07-6.37, p<0.001$ ), and also with 12 -month quit success $(\mathrm{OR}=1.85,95 \% \mathrm{Cl}=1.14-3.00, p=0.013)$. The support of other colleagues (not participating in the smoking cessation program) was not significantly associated with quit success at either time. Having very supportive friends compared to not very supportive friends was not significantly associated with short-term or 12-month quit success. Finally, having a very supportive partner was positively associated with quit success $(\mathrm{OR}=2.01,95 \% \mathrm{Cl}=1.23-3.30, p=0.006)$, but only directly after finishing 
the training and not after 12 months. The results of the separate analyses of the social influence of the social environment that were performed to select variables to include in the multivariable analysis showed effects comparable to the multivariable analysis (Appendix A).

\section{Associations of Smoking Behavior of Persons in the Social Environment with Quit Success}

Among the participants' five closest friends, acquaintances or colleagues, the mean number of smokers was 3.8 (1.5) (Table 2). A larger number of smokers among these five persons was negatively associated with quit success (Figure 1 and Table 3). This effect was not statistically significant directly after the smoking cessation training program, but was significant 12 months after finishing the program $(\mathrm{OR}=0.81,95 \% \mathrm{Cl}=0.71-0.92$, $p=0.002$ ). This result means that with each extra smoker within someone's close circle of five people, the odds of continuous smoking cessation after 12 months decreased by approximately $19 \%$. Of the participants who had a partner, most had a partner who had never smoked (58\%), $26 \%$ had a partner who smoked and $15 \%$ had a partner who had quit smoking between baseline and the end of the smoking cessation program. The smoking status of the partner was also associated with quit success. Compared to having a partner that had never smoked, having no partner was significantly and negatively associated with quit success directly after finishing the smoking cessation training (OR = $0.46,95 \% \mathrm{Cl}=0.25-0.85, p=0.014)$. The partner's smoking status during the quit attempt was also significantly associated with 12-month quit success. Compared to participants who had a partner that had never smoked, all other partner categories (a partner who continued smoking, a partner who had stopped smoking, having no partner or other) were significantly and negatively associated with successful quitting. The results of the separate analyses of the smoking behavior of persons in the social environment that were performed to select variables to include in the multivariable analysis showed comparable effects to the multivariable analysis (Appendix A).

\section{Sensitivity Analyses}

The sensitivity analyses where only complete cases were included showed comparable results to the main analyses of the impact of the smoking behavior of and social support from people in the social environment on short-term as well as long-term smoking cessation success (Appendix B). 


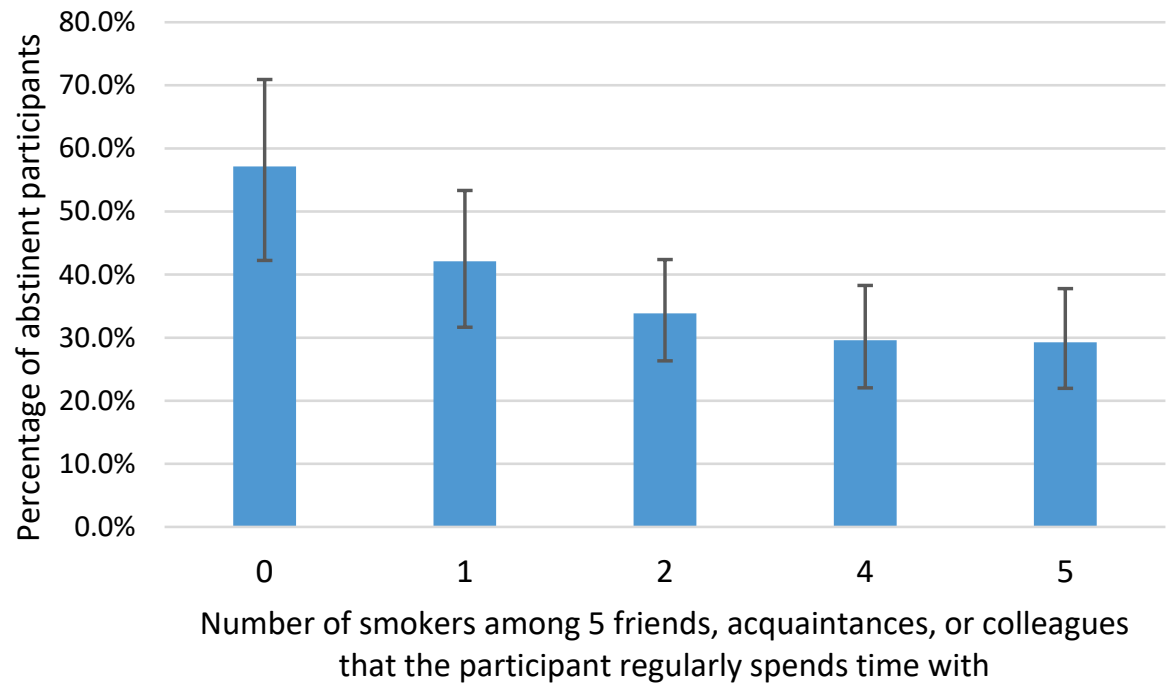

Figure 1. Percentage (95\% confidence interval $(\mathrm{Cl})$ ) of participants that continuously abstained from smoking up to 12 months after finishing the smoking cessation program, presented for each number of smokers among the five closest friends, acquaintances, or colleagues that the participant regularly spends time with. 
Table 3. Associations of smoking behavior in the social environment and social support with shortand long-term smoking abstinence.

\begin{tabular}{|c|c|c|c|c|c|c|}
\hline \multirow{2}{*}{ Variable } & \multicolumn{3}{|c|}{$\begin{array}{l}\text { Smoking abstinence directly after } \\
\text { the smoking cessation program }{ }^{A}\end{array}$} & \multicolumn{3}{|c|}{$\begin{array}{c}\text { Smoking abstinence } 12 \text { months } \\
\text { after the smoking cessation } \\
\text { program }{ }^{\mathrm{B}}\end{array}$} \\
\hline & $\begin{array}{l}\text { Odds } \\
\text { Ratio }\end{array}$ & $\begin{array}{c}95 \% \\
\text { Confidence } \\
\text { Interval }\end{array}$ & $p$ value & $\begin{array}{l}\text { Odds } \\
\text { Ratio }\end{array}$ & $\begin{array}{l}95 \% \\
\text { Confidence } \\
\text { Interval }\end{array}$ & $p$ value \\
\hline $\begin{array}{l}\text { Number of smokers in close } \\
\text { circle }(0-5)\end{array}$ & 0.93 & $0.80-1.08$ & 0.346 & 0.81 & $0.71-0.92$ & $0.002 *$ \\
\hline \multicolumn{7}{|l|}{ Partner smoking status } \\
\hline Continued smoking & 0.62 & $0.33-1.17$ & 0.140 & 0.40 & $0.24-0.66$ & $<0.001 *$ \\
\hline Stopped & 0.87 & $0.36-2.06$ & 0.745 & 0.47 & $0.26-0.86$ & $0.014 *$ \\
\hline No partner & 0.46 & $0.25-0.85$ & $0.014 *$ & 0.48 & $0.26-0.88$ & $0.019 *$ \\
\hline Other & 0.23 & $0.09-0.57$ & $0.002 *$ & 0.09 & $0.02-0.35$ & $<0.001 *$ \\
\hline \multicolumn{7}{|l|}{$\begin{array}{l}\text { Group training colleague } \\
\text { support }\end{array}$} \\
\hline $\begin{array}{l}\text { Not very supportive (ret.) } \\
\text { Very supportive }\end{array}$ & 3.63 & $2.07-6.37$ & $<0.001$ * & 1.85 & $1.14-3.00$ & $0.013 *$ \\
\hline $\begin{array}{l}\text { Other colleague support } \\
\text { Not very supportive (ref.) } \\
\text { Very supportive }\end{array}$ & $\begin{array}{c}1 \\
0.95\end{array}$ & $0.57-1.58$ & 0.850 & n.s. ${ }^{c}$ & n.s. ${ }^{c}$ & n.s. ${ }^{c}$ \\
\hline $\begin{array}{l}\text { Friends and family support } \\
\text { Not very supportive (ref.) } \\
\text { Very supportive }\end{array}$ & $\begin{array}{c}1 \\
1.23\end{array}$ & $0.76-1.99$ & 0.398 & n.s. ${ }^{c}$ & n.s. ${ }^{c}$ & n.s. ${ }^{c}$ \\
\hline $\begin{array}{l}\text { Partner support } \\
\text { Not very supportive or no } \\
\text { partner (ref.) }\end{array}$ & 1 & & & 1 & & \\
\hline Very supportive & 2.01 & $1.23-3.30$ & $0.006 *$ & 1.19 & $0.75-1.88$ & 0.465 \\
\hline
\end{tabular}

The dependent outcome variable was CO-validated continuous smoking abstinence. All analyses were controlled for intervention group, educational level, income level, and Fagerström score. Multiple imputation was used for missing values and a random intercept at company level was used to adjust for the clustering of participants within a company. ${ }^{A}$ In this analysis, separate logistic regression analyses were used for each individual variable, still including all control variables. ${ }^{B}$ In this multivariable analysis, a preselection was made first by comparing all variables separately with only the control variables in the model (see Appendix A, multiple imputation). The variables with a $p$ value lower than 0.200 were then selected and included in the multivariable analysis. ${ }^{C}$ These variables were non-significant in the preselection analysis (see Appendix $A$, multiple imputation) and therefore not included in the 12-month analysis. ${ }^{*} p \leq 0.05$. 


\section{DISCUSSION}

In the current study, we aimed to investigate whether social support from, and the smoking behavior of colleagues, partners, and family and friends of employees who participated in a workplace smoking cessation training program were associated with short- and longterm quit success.

The results of the current study confirm our hypothesis that colleagues have an important influence on smoking cessation success. Not only did most participants (78\%) find their colleagues who also participated in the smoking cessation group training very supportive, but also support from these colleagues was significantly associated with quit success. Having a very supportive partner was also positively associated with short-term quit success, while support from friends and family and other colleagues was not significantly associated with quit success. These results suggest that people who are closely involved in the quitting process of the smoker are especially important. An interesting finding was that unlike partner support, social support from colleagues who participated in the training was associated with a higher probability of smoking cessation up to 12 months after finishing the training. Previous research had suggested that social support mainly influences the achievement of abstinence, not the continuation of abstinence in the long term. ${ }^{5,19,20}$ However, the lack of an effect of partner support on long-term quit success may also be explained because the actual smoking behavior of the partner rather than the support may be ultimately decisive for quit success. Likewise, the smoking behavior of colleagues may be particularly influential. ${ }^{1}$ Therefore, the effect of social support from colleagues should be explored further while considering the smoking behavior of these colleagues. The positive association of colleague support with smoking cessation found in the current study underlines the importance of social processes, such as peer support and peer pressure, that are unique to group counselling as compared to individual treatment. This potential beneficial effect of colleagues on quit success is interesting from a health promotion perspective; it suggests that the work environment may be a promising environment for interventions, perhaps particularly for smokers in whose home environment smoking is more prevalent and socially accepted.

Our study indicated that the number of other smokers in the social environment has a large effect on the success of people who want to quit smoking. This result confirms the influence of smokers in the social environment found in previous research, $1,2,20$ and is comparable with an earlier study that showed an increase in relapse odds of 1.12 for each friend who smoked. ${ }^{26}$ Additionally, that particular study found that a higher proportion of smokers among friends was associated with relapse only approximately one month after quitting, which is in line with our results showing that the number of smokers was not significantly associated with quit success directly after finishing the training, but was associated with quit success at 12 months. This may be explained because the participants feel more vulnerable in the first phase of quitting smoking and may avoid social situations that could lead to relapse, while in a later phase participants may feel more secure about their abstinence and may be less cautious. The findings of the current study imply that smoking cessation counsellors should evaluate whether there are many smokers in the quitter's social environment and aim to overcome corresponding barriers to quit success. 
According to our results and previous research, ${ }^{6,8}$ having a partner who has never smoked seems to be a factor that can prevent smoking relapse. Compared to having a partner that never smoked, having no partner was negatively associated with short-term quit success. The negative influence of not having a partner has also been shown in other studies ${ }^{6,9}$ An explanation could be that quitters benefit from having someone who monitors their abstinence and provides social control in the first difficult phase of quitting smoking. A partner could also help maintain a high motivation to stay abstinent. The association of the smoking status of the partner during the smoking cessation program with 12-month quit success was also prominent. Compared to having a partner who had never smoked, having no partner, having a partner who smoked, and even having a partner who had quit smoking substantially decreased the probability of quit success. These results confirm studies showing the effect of partners on smoking cessation and continuous abstinence. $1,6,27$ For successful cessation treatments, it may be important to also include the partner in the quitting process.

\section{Strengths and Limitations}

A strength of the current study is that it showed a potentially very effective source of social support that can be used to improve quit success in smokers, namely the support of colleagues who participated in a workplace group smoking cessation program alongside the smoker. Further research should explore whether the positive association of peer support with quit success can be generalized to general population settings. The current study was a post hoc analysis, which implies that research questions and hypotheses were made after the data were collected. Further research is therefore needed to confirm our findings. Another limitation of the current study is that while we investigated the influence of the smoking behavior of the social environment and social support between baseline and the end of the smoking cessation program, we did not assess the influence of changes in these variables within the remainder of the 12-month follow-up period. Furthermore, data on the smoking behavior of partners were self-reported by participants, so they may not be accurate if the participants misclassified their partner's smoking status. However, it can be argued for the current study that the smoking status of the partner as perceived by the participant is more relevant than the partner's objective smoking behavior. In addition, in order to determine the smoking status or change in smoking status of the partner between baseline and after finishing the smoking cessation training (a period of eight weeks), limitations in the data forced us to assume that these partners were still the same individuals, while it is possible that some participants changed partners in between. Moreover, because few participants had relapsed into smoking at the follow-up measurement directly after completion of the program, we performed separate analyses to assess the associations of the independent variables with quit success to prevent unreliable results. For the outcome "quit success directly after completion of the program", we were, therefore, unable to determine whether variables would still be independently associated with quit success if all variables were included in the model. Finally, the amount of missing data that needed to be imputed was rather high for some variables, and there were differences in some variables between participants with and without missing values, which could indicate selection bias. However, the complete case analysis showed conclusions similar to the main analysis. 


\section{Conclusions}

In the current study, social support from colleagues was strongly associated with the longterm quit success of employees who participated in a group smoking cessation training program at the workplace. Whether a quit attempt is successful may also depend on the smoking behavior of the partner and the proportion of smokers in the social environment. The current study implies that it is desirable to include the social environment in the smoking cessation process in order to increase the probability of quit success. The workplace can be a favorable setting for smoking cessation interventions, as colleagues may provide a valuable source of support. 


\section{REFERENCES}

1. Christakis NA, Fowler JH. The collective dynamics of smoking in a large social network. N Engl J Med. 2008;358(21):2249-2258.

2. Hitchman SC, Fong GT, Zanna MP, Thrasher JF, Laux FL. The relation between number of smoking friends, and quit intentions, attempts, and success: findings from the International Tobacco Control (ITC) Four Country Survey. Psychol Addict Behav. 2014;28(4):1144-1152.

3. Holahan CK, Holahan CJ, Li X, Jung SJ. Social influences on smoking in American workers: the role of the presence of smokers in the workplace and in the home. Am J Health Promot. 2013;28(2):105-107.

4. Stead LF, Carroll AJ, Lancaster T. Group behaviour therapy programmes for smoking cessation. Cochrane Database of Systematic Reviews. 2017(3).

5. Lawhon D, Humfleet GL, Hall SM, Munoz RF, Reus VI. Longitudinal analysis of abstinence-specific social support and smoking cessation. Health Psychol. 2009;28(4):465-472.

6. Margolis R, Wright L. Better off alone than with a smoker: the influence of partner's smoking behavior in later life J Gerontol B Pscyhol Sci Soc Sci. 2016;74(4):687-697.

7. Homish GG, Leonard KE. Spousal influence on smoking behaviors in a US community sample of newly married couples. Social science \& medicine 2005;61(12):2557-2567.

8. Chandola T, Head J, Bartley MJ. Socio-demographic predictors of quitting smoking: how important are household factors? Addiction. 2004;99(6):770-777.

9. Ferguson J, Bauld L, Chesterman J, Judge K. The English smoking treatment services: one-year outcomes. Addiction. 2005;100(s2):59-69.

10. Hyland A, Li Q, Bauer JE, Giovino GA, Steger C, Cummings KMJ. Predictors of cessation in a cohort of current and former smokers followed over 13 years. Nicotine \& Tobacco Research2004;6(Suppl_3):S363-S369.

11. Biener L, Hamilton WL, Siegel M, Sullivan EM. Individual, social-normative, and policy predictors of smoking cessation: A multilevel longitudinal analysis. Am J Public Health. 2010;100(3):547-554.

12. Baha M, Le Faou AL. Smokers' reasons for quitting in an anti-smoking social context. Public Health. 2010;124(4):225-231.

13. Westmaas JL, Bontemps-Jones J, Bauer JE. Social support in smoking cessation: Reconciling theory and evidence. Nicotine \& Tobacco Research. 2010;12(7):695-707.

14. vanDellen MR, Boyd SM, Ranby KW, MacKillop J, Lipkus IMJ. Willingness to provide support for a quit attempt: A study of partners of smokers. J Health Psychol. 2016;21(9):1840-1849.

15. Shiffmann S. Dynamic influences on smoking relapse process. J Pers. 2005;73(6):1715-1748.

16. Quist HG, Christensen U, Carneiro IG, Hansen JV, Bjorner JB. Do colleagues influence our lifestyle: the matter of smoking, body mass index and leisure-time physical activity? Prev Med. 2014;67:166-170.

17. Yong LC, Luckhaupt SE, Li J, Calvert GM. Quit interest, quit attempt and recent cigarette smoking cessation in the US working population, 2010. Occup Environ Med. 2014;71(6):405-414.

18. Van den Brand FA, Dohmen LME, Van Schayck CP, Nagelhout GE. 'Secretly, it's a competition': a qualitative study investigating what helped employees quit smoking during a workplace smoking cessation group training programme with incentives. BMC Open. 2018;8(11).

19. Roski J, Schmid LA, Lando HA. Long-term associations of helpful and harmful spousal behaviors with smoking cessation. Addict Behav. 1996;21(2):173-185.

20. Mermelstein R, Cohen S, Lichtenstein E, Baer JS, Kamarck T. Social support and smoking cessation and maintenance. J Consult Clin Psychol. 1986;54(4):447-453. 
21. van den Brand FA, Nagelhout GE, Winkens B, et al. The effect of financial incentives on top of behavioral support on quit rates in tobacco smoking employees: study protocol of a clusterrandomized trial. BMC Public Health. 2016;16(1):1056.

22. van den Brand FA, Nagelhout GE, Winkens B, Chavannes NH, van Schayck OCP. Effect of a workplace-based group training programme combined with financial incentives on smoking cessation: a cluster-randomised controlled trial. Lancet Public Health. 2018;3(11):e536-e544. doi:10.1016/S2468-2667(18)30185-3.

23. West RPLJ. Outcome criteria in smoking cessation trials: proposal for a common standard. Addiction. 2005;100.

24. Heatherton TF, Kozlowski LT, Frecker RC, Fagerstrom K-O. The Fagerström Test for Nicotine Dependence: a revision of the Fagerstrom Tolerance Questionnaire. Br J Addict. 1991;86(9):11191127.

25. Fagerström K, Furberg H. A comparison of the Fagerström Test for Nicotine Dependence and smoking prevalence across countries. Addiction. 2008;103(5):841-845.

26. Herd N, Borland R, Hyland A. Predictors of smoking relapse by duration of abstinence: findings from the International Tobacco Control (ITC) Four Country Survey. Addiction. 2009;104(12):20882099.

27. Dollar KM, Homish GG, Kozlowski LT, Leonard KE. Spousal and alcohol-related predictors of smoking cessation: a longitudinal study in a community sample of married couples. Am J Public Health. 2009;99(2):231-233. 


\section{Appendix A}

Associations of smoking behavior in the social environment and social support with 12-month smoking cessation: univariable analysis.

\begin{tabular}{|c|c|c|c|c|c|c|}
\hline \multirow[b]{2}{*}{ Variable } & \multicolumn{3}{|c|}{ Multiple imputation } & \multicolumn{3}{|c|}{ Complete case analysis } \\
\hline & $\begin{array}{l}\text { Odds } \\
\text { Ratio }\end{array}$ & $\begin{array}{c}95 \% \\
\text { Confidence } \\
\text { Interval } \\
\end{array}$ & p value & $\begin{array}{l}\text { Odds } \\
\text { Ratio }\end{array}$ & $95 \% \mathrm{Cl}$ & p value \\
\hline $\begin{array}{l}\text { Number of smokers in close circle } \\
(0-5)\end{array}$ & 0.79 & $0.69-0.89$ & $<0.001 *$ & 0.80 & $0.70-0.92$ & 0.002 \\
\hline $\begin{array}{l}\text { Partner smoking status } \\
\text { Never smoked (ref.) }\end{array}$ & 1 & & & 1 & & \\
\hline Continued smoking & 0.36 & $0.22-0.59$ & $<0.001 *$ & 0.43 & $0.25-0.73$ & $0.002 *$ \\
\hline Stopped & 0.47 & $0.26-0.84$ & $0.012 *$ & 0.53 & $0.27-1.02$ & 0.059 \\
\hline No partner & 0.39 & $0.23-0.65$ & $<0.001 *$ & 0.37 & $0.21-0.66$ & $<0.001 *$ \\
\hline Other & 0.08 & $0.02-0.33$ & $<0.001 *$ & 0.04 & $0.01-0.31$ & $0.002 *$ \\
\hline $\begin{array}{l}\text { Group training colleague support } \\
\text { Not very supportive (ref.) }\end{array}$ & 1 & & & 1 & & \\
\hline Very supportive & 1.94 & $1.22-3.10$ & $0.006 *$ & 1.25 & $0.75-2.07$ & 0.392 \\
\hline $\begin{array}{l}\text { Other colleague support } \\
\text { Not very supportive (ref.) }\end{array}$ & 1 & & & 1 & & \\
\hline Very supportive & 0.83 & $0.56-1.21$ & 0.328 & 0.74 & $0.48-1.14$ & 0.172 \\
\hline $\begin{array}{l}\text { Friends and family support } \\
\text { Not very supportive (ref.) } \\
\text { Very supportive }\end{array}$ & $\begin{array}{c}1 \\
1.11\end{array}$ & $0.77-1.61$ & 0.566 & $\begin{array}{c}1 \\
1.02\end{array}$ & $0.68-1.54$ & 0.915 \\
\hline $\begin{array}{l}\text { Partner support } \\
\text { Not very supportive or no } \\
\text { partner (ref.) } \\
\text { Very supportive }\end{array}$ & $\begin{array}{c}1 \\
1.88\end{array}$ & $1.30-2.70$ & $>0.001 *$ & $\begin{array}{c}1 \\
1.66\end{array}$ & $1.10-2.51$ & $0.016 *$ \\
\hline
\end{tabular}

The dependent outcome variable was CO-validated continuous smoking abstinence. Separate logistic regression analyses were used for each individual variable. All analyses were controlled for intervention group, educational level, income level, and Fagerström score, and a random intercept at company level was used to adjust for the clustering of participants within a company. ${ }^{*} p \leq 0.05$. 


\section{Appendix B}

Associations of smoking behavior in the social environment and social support with short and long-term smoking cessation: complete case analysis.

\begin{tabular}{|c|c|c|c|c|c|c|}
\hline \multirow[t]{2}{*}{ Variable } & \multicolumn{3}{|c|}{$\begin{array}{l}\text { Directly after smoking } \\
\text { cessation program }{ }^{A}\end{array}$} & \multicolumn{3}{|c|}{$\begin{array}{c}12 \text { months after smoking cessation } \\
\text { program }^{B}\end{array}$} \\
\hline & $\begin{array}{l}\text { Odds } \\
\text { Ratio }\end{array}$ & $\begin{array}{c}95 \% \\
\text { Confidence } \\
\text { Interval } \\
\end{array}$ & p value & Odds Ratio & $\begin{array}{l}\text { 95\% Confidence } \\
\text { Interval }\end{array}$ & p value \\
\hline $\begin{array}{l}\text { Number of smokers in close } \\
\text { circle }(0-5)\end{array}$ & 0.93 & $0.79-1.10$ & 0.390 & 0.81 & $0.69-0.94$ & $0.006^{*}$ \\
\hline $\begin{array}{l}\text { Partner smoking status } \\
\text { Never smoked (ref.) } \\
\text { Continued smoking } \\
\text { Stopped } \\
\text { No partner } \\
\text { Other }\end{array}$ & $\begin{array}{c}1 \\
1.09 \\
1.35 \\
0.46 \\
0.39\end{array}$ & $\begin{array}{l}0.50-2.37 \\
0.48-3.83 \\
0.24-0.89 \\
0.13-1.16\end{array}$ & $\begin{array}{c}0.828 \\
0.573 \\
0.022^{*} \\
0.090\end{array}$ & $\begin{array}{c}1 \\
0.49 \\
0.58 \\
0.46 \\
0.05\end{array}$ & $\begin{array}{l}0.28-0.86 \\
0.29-1.17 \\
0.23-0.91 \\
0.01-0.38\end{array}$ & $\begin{array}{r}0.013 * \\
0.127 \\
0.027 * \\
0.004 *\end{array}$ \\
\hline $\begin{array}{l}\text { Group training colleague } \\
\text { support } \\
\text { Not very supportive (ref.) } \\
\text { Very supportive }\end{array}$ & $\begin{array}{c}1 \\
3.53\end{array}$ & $1.94-6.42$ & $<0.001 *$ & $\begin{array}{c}1 \\
1.24\end{array}$ & $0.73-2.11$ & 0.422 \\
\hline $\begin{array}{l}\text { Other colleague support } \\
\text { Not very supportive (ref.) } \\
\text { Very supportive }\end{array}$ & $\begin{array}{c}1 \\
0.98\end{array}$ & $0.55-1.73$ & 0.936 & n.s. & n.s. & n.s. \\
\hline $\begin{array}{l}\text { Friends and family support } \\
\text { Not very supportive (ref.) } \\
\text { Very supportive }\end{array}$ & $\begin{array}{c}1 \\
1.26\end{array}$ & $0.72-2.20$ & 0.412 & n.s. & n.s. & n.s. \\
\hline $\begin{array}{l}\text { Partner support } \\
\text { Not very supportive or no } \\
\text { partner (ref.) } \\
\text { Very supportive }\end{array}$ & $\begin{array}{c}1 \\
2.07\end{array}$ & $1.17-3.66$ & $0.012^{*}$ & $\begin{array}{c}1 \\
1.11\end{array}$ & $0.66-1.87$ & 0.700 \\
\hline
\end{tabular}

The dependent outcome variable was $\mathrm{CO}$-validated continuous smoking abstinence. All analyses were controlled for intervention group, educational level, income level, and Fagerström score. Multiple imputation was used for missing values and a random intercept at company level was used to adjust for the clustering of participants within a company. ${ }^{\mathrm{A}}$ In this analysis, separate logistic regression analyses were used for each individual variable. ${ }^{B}$ In this multivariable analysis, all variables were separately assessed with only the control variables in the model (appendix $A$, complete case analysis), and then the variables with a $P$ value lower than 0.200 were selected and included in the multivariable analysis. ${ }^{*} p \leq 0.0$ 



\section{CHAPTER 8}

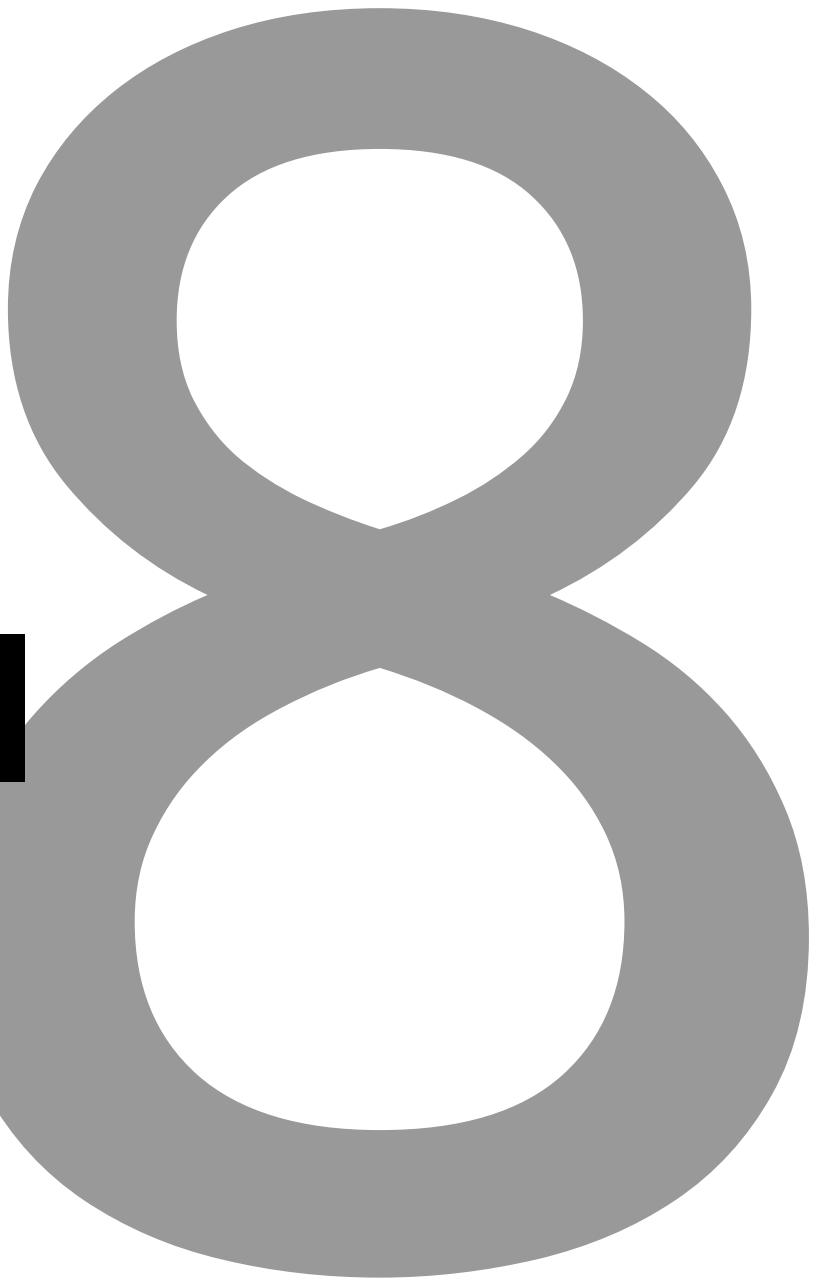




\section{How financial incentives increase smoking cessation: a two-level path analysis}

Van den Brand FA, Candel MJJM, Nagelhout GE, Winkens B, van Schayck CP. How Financial Incentives Increase Smoking Cessation: A Two-Level Path Analysis 


\section{ABSTRACT}

Introduction Financial incentives effectively increase smoking cessation rates, but it is unclear via which psychological mechanisms incentives influence quit behavior. The current study examines how receiving financial incentives for smoking cessation leads to quitting smoking and investigates several mediators and moderators of that relationship.

Methods The study sample consisted of 604 tobacco-smoking employees from 61 companies in the Netherlands who completed a baseline and follow-up questionnaire. The current study is a secondary analysis from a cluster randomized trial (CRT) where employees received smoking cessation group counselling at the workplace. Participants in the intervention group additionally received financial incentives of $€ 350$ in total for 12-month continuous smoking abstinence. We used a two-level path analysis to test a model that assesses the effects of financial incentives through smoking cessation program evaluation, medication use, nicotine replacement use, attitudes, self-efficacy and social influences on quit success. We additionally tested whether an individual's reward responsiveness moderated the influence of incentives on quit success.

Results The effect of financial incentives on quit success was mediated by a higher self-efficacy. Financial incentives were also associated with a higher use of cessation medication. A more positive program evaluation was related to higher self-efficacy, more social influence to quit, and more positive attitudes about quitting. The results did not differ significantly by individual reward responsiveness.

Conclusions The results of the current study suggest that financial incentives may be used to increase medication use and self-efficacy for quitting smoking, which offers an indirect way to increase successful smoking cessation. 


\section{INTRODUCTION}

Financial incentives are used to promote health-related behavior or to motivate behavior change in different fields, including smoking cessation. ${ }^{1-4}$ Although financial incentives for smoking cessation have shown to increase quit rates in a growing body of research, it is not exactly known how incentives work. It has been suggested that incentives are effective because they provide an attainable goal in the near future ${ }^{5}$ and because providing financial incentives is a form of operant conditioning that can lead to voluntary changes in habitual behavior. ${ }^{6}$ However, it is not clear via which psychological mechanisms financial incentives influence quit behavior, and via which pathways incentives lead to an increase in smoking cessation success. ${ }^{7-10}$ A better understanding of the workings of financial incentives can help to design effective interventions to reduce smoking prevalence, as it can provide insight into which other elements within an intervention with financial incentives can be adapted to improve the intervention's effect. For example, financial incentives may, apart from increasing quit success directly, have a positive effect on other factors that are known to increase quit success, such as a higher willingness to use smoking cessation medications or nicotine replacement therapy (NRT). This could subsequently be a reason to make these medications easily accessible together with an intervention with financial incentives. ${ }^{11}$ In a previous study, we performed a cluster randomized trial (CRT) where employees participated in a smoking cessation group training organized at the workplace., ${ }^{912}$ Participants in the intervention group earned vouchers if they succeeded at quitting smoking, which resulted in significantly higher quit rates after twelve months in the intervention group compared to the control group. ${ }^{9}$ In the current study, we will test a model of the psychological pathways leading from financial incentives to quit success via smoking cessation training programme evaluation, smoking cessation medication use, NRT use, and the three psychosocial mediators attitude, social influence, and selfefficacy, known from several behavioural change theories such as the ASE (Attitude, Social influence, Efficacy)-model ${ }^{13}$ and Theory of Planned Behavior ${ }^{14}$

Financial incentives could increase the probability that a person successfully quits smoking by enhancing motivation to quit, ${ }^{15}$ but could also work through different mediators, for example if incentives stimulate smokers to make use of a smoking cessation treatment. ${ }^{11}$ In a CRT where incentives for quit success were offered without any cessation support, participants in the incentives group reported more often that they obtained help with quitting smoking on the internet. ${ }^{16}$ In a different study, incentives stimulated people to call a quit line more often. ${ }^{17}$ Additionally, a qualitative study among pregnant smokers based in the United Kingdom found that pregnant women appeared to have used the National Health Service (NHS) Smoking Services more because of incentives and suggested that the positive affect created by the incentives may have caused the women to regard the Smoking Services more positively. ${ }^{18}$ In the current study, we test the idea that because of financial incentives, people may be more positive toward participating in a smoking cessation training programme, which may increase their engagement with the programme and could, in turn, enhance its effectiveness. Likewise, we examine whether financial incentives for quit success may make people more inclined to use smoking cessation medications to improve their chances of quitting smoking, which can indirectly lead to higher success rates. ${ }^{19}$ 
The effect of financial incentives on quit success may furthermore run through the psychosocial mediators attitudes, social influences and self-efficacy, which predict a person's intention to change their behaviour and can lead to behavioural change such as quitting smoking. ${ }^{13,14,20,21}$ To our best knowledge, the current study is the first to examine whether these psychosocial factors mediate the effect of financial incentives on smoking cessation, which leaves us to speculate about possible mechanisms. For example, participants may like the idea of being able to earn rewards for quitting smoking and enjoy receiving the rewards, ${ }^{15,22}$ which may contribute to a positive attitude toward quitting smoking. Social influences from a smoker's environment include the subjective norm toward smoking, the perceived social pressure to quit smoking, and social support for quitting smoking. ${ }^{20}$ When financial incentives are provided for quitting smoking this may propagate a social norm of non-smoking, ${ }^{20}$ and smokers may receive more encouragement from people in their social environment to reach their goal of quitting smoking and receiving the reward, for example when a spouse also profits from the reward. ${ }^{23}$ Finally, financial incentives may increase a person's self-efficacy to quit smoking, for example if they stimulate visualization of success. ${ }^{24}$

The effect of financial incentives on motivating behavior change may differ among individuals. ${ }^{15}$ It may be influenced by how attractive a specific incentive is to an individual. ${ }^{22}$ The effect of incentives could also depend on how sensitive a person is to rewards in general. ${ }^{25}$ Greater perceived importance of receiving incentives for abstinence has been associated with increased smoking cessation success in a previous study. ${ }^{26}$ The effect of financial incentives on quit success may therefore be stronger for individuals who are more responsive to rewards. ${ }^{25,27}$

The current study examines how receiving financial incentives for smoking cessation leads to quitting smoking and investigates several mediators of that relationship. We hypothesize that financial incentives influence smoking cessation via a more positive appraisal of the smoking cessation training programme, higher medication and NRT use and through a positive effect on the psychosocial mediators attitudes, self-efficacy and social influence. Finally, we hypothesize that these associations are dependent on an individual's responsiveness to rewards.

\section{METHODS}

\section{Design}

The data used for this study are from a CRT performed within Dutch companies, of which the methods and results have been described in more detail in previous publications. ${ }^{9,12}$ In the cluster randomized trial, 604 employees from 61 companies received the same group smoking cessation training program at their workplace which consisted of seven weekly sessions of 90 minutes. The group training program was provided by the Dutch company SineFuma. The training program has various components that help participants to quit smoking, and includes enhancing motivation to quit smoking, creating positive attitudes for quitting, and improving self-efficacy for quitting smoking and maintaining abstinence. The group component stimulates social support, peer pressure and a positive social 
norm toward quitting smoking. Finally, the program increases knowledge about nicotine addiction, the positive effects of quitting smoking, and provides information on smoking cessation medications. After cluster randomization, 31 companies (320 employees) were allocated to the intervention group and 30 companies (284 employees) to the control group. Employees from companies in the intervention group earned vouchers of $€ 350$ in total if they had quit smoking successfully. All employees were aware of the incentives before the start of the program, but the result of the randomization was unknown to participants up until it was revealed during the first training session. Data were collected between March 2016 and March 2018 via online questionnaires, which were distributed at baseline, at the first follow-up measurement which was directly after finishing the training program, and at 3 months, 6 months and 12 months after the training program. The study was registered in the Netherlands Trial Register (NL5537) and cleared by the medicalethical committee METC Z in Heerlen, The Netherlands.

\section{Participants}

All participants from the CRT were included in the current study $(n=604)$. Participants were employees from Dutch companies of at least 18 years old who smoked tobacco. There were no exclusion criteria regarding the amount of tobacco that the participants smoked.

\section{Measurements}

\section{Control variables}

Control variables were gender, age, highest completed educational level, income level, and nicotine dependence. The highest completed educational level was divided into three categories: low (none completed, primary school, lower secondary education), moderate (middle secondary education) and high (upper secondary education, university). Income was based on individualized net household income and divided into three groups based on tertiles ( $€ 0-€ 1630, € 1630-€ 2210, € 2210$ and higher). Nicotine dependence was measured using the Fagerström score ranging from 0 to 10 where higher scores indicate a higher nicotine dependence. ${ }^{28}$

\section{Financial incentives}

Participants in the intervention group received vouchers for continuous abstinence from smoking, validated by expired-air carbon monoxide (CO) measurement. CO-measurements were performed and vouchers were distributed upon abstinence directly after finishing the smoking cessation program $(€ 50)$, after three months (€50), after six months $(€ 50)$, and after 12 months (€200). Participants in the control group did not receive incentives. The variable financial incentives was categorized as "incentives group" (1) and "control group" (0).

\section{Smoking cessation group training program}

As a measure for appraisal of the group training program, we assessed how participants evaluated the program using 13 questions (see Supplementary file 1) that were specifically designed for the current study, which each could be answered on a 5-point Likert scale from 1 (completely disagree) to 5 (completely agree). The questions concerned the quality, 
content, and duration of the training, the communication with the trainer, and whether participants liked and were satisfied with the program and would recommend it to others. Additionally, we asked the participants to grade the training with a score ranging from 1 (worst) to 10 (best). The scores on the evaluation items were averaged into a single scale score, where the grade score was first rescaled into a range from 1 to 5 . Cronbach's Alpha was 0.86 .

\section{Medication and nicotine replacement therapy}

Participants could fill out whether they had used smoking cessation medication or NRT during their quit attempt. Both medication use and NRT were coded as "yes" (1) or "no" (0), indicating whether the participant used at least one medication or NRT for smoking cessation.

\section{Psychosocial mediators}

Attitudes about smoking cessation were measured with the question: "If you quit smoking within the next 3 months, this would be..." for participants who were smokers, and with the question "If you stay abstinent from smoking for the next 3 months, this would be..." for participants who had quit smoking. Participants could answer on three 5-point scales if they thought this would be wise or unwise, pleasant or unpleasant and positive or negative. ${ }^{20}$ Cronbach's Alpha was 0.67 . The scale score, being the average of the item scores, was used for the analysis. Self-efficacy for quitting smoking was assessed by asking: "Suppose you want to quit smoking within the next 3 months (first part was presented only to smokers), will you be able to resist smoking when: (1) you just woke up? (2) you have experienced something annoying? (3) you are having a cup of coffee or tea? (4) you are drinking alcohol? (5) you are offered a cigarette?" 29 Response options ranged on a 5-point scale from "I will certainly not be able" (1) to "I will certainly be able" (5). Cronbach's Alpha was 0.79 . The average of the item scores was the scale score and was used in the analysis.

Social influence was measured by the subjective norm toward quitting smoking and social support for quitting smoking. Subjective norm was measured with the question: "How do you think that people who are important to you would feel if you did not smoke in the next three months?" Participants could answer this question on a 5-point scale from "strongly disapprove" (1) to "strongly approve" (5)..$^{20}$ Social support that participants received from (a) their partner, (b) friends and family, (c) colleagues who also participated in the group smoking cessation program, and (d) colleagues who did not participate in the cessation program was assessed with the question: "How supportive do you think your [ad] would be if you attempted to quit smoking?" Response options were "very supportive" (3), "moderately supportive"(2), "little supportive" (1) and "don't know". The "don't know" category was classified as missing. Cronbach's Alpha was 0.62 . The scale score, being the average of the item scores, was used for the analysis. 


\section{Reward responsiveness}

Reward responsiveness was measured using the Behavior Activation System (BAS) Reward Responsiveness Scale consisting of five statements that participants could agree or disagree with, ${ }^{30}$ for example: "When I get something I want, I feel excited and energized". Response options consisted of a 4-point scale ranging from "very false for me" (1) to "very true for me" (4). A higher score on an item indicates a higher reward responsiveness. Cronbach's Alpha was 0.75 . The average of the item scores yielded the scale score, which was used in the analysis.

\section{Quit success}

Quit success was defined as carbon monoxide (CO) validated continuous smoking abstinence 12 months after finishing the training program. A research assistant made appointments to visit participants at the workplace who reported to be abstinent from smoking to biochemically validate smoking abstinence using CO-measurement. Over the 12-month follow-up period, CO-measurements were performed directly after finishing the smoking cessation program, and 3, 6 and 12 months after finishing the program. If COvalidation could not be performed, for example due to an employee's illness or vacation, or if the CO measurement exceeded the threshold of 9 parts per million (ppm) ${ }^{31}$, the participant was considered to be a smoker.

\section{Statistical analyses}

IBM SPSS Statistics for Windows (version 25.0, Armonk, NY: IBM Corp.) was used for analyses of sample characteristics, loss to follow up, reliability of scales, and correlations. Mplus version 7.3 was used to perform the two-level path analysis. We assessed model fit using the Comparative Fit Index (CFI), the Tucker-Lewis Index (TLI), and the Root-MeanSquare Error of Approximation (RMSEA). We consider the model to be a good fit if the CFI and TLI were $>0.90$ and the RMSEA was $<0.05 .^{32}$

We first tested a model of the effect of financial incentives for smoking cessation via both a direct pathway on 12-month smoking abstinence, and via the psychosocial mediators attitudes, self-efficacy and social influence, group training evaluation, medication use and NRT use. We declared a path between financial incentives and quit success statistically significant if all intermediate associations of a path were also significant (Joint Significance Test). ${ }^{33}$ We tested a second model to examine whether reward responsiveness moderated the effect of incentives. We adjusted the analyses for the first and the second model for all control variables mentioned in the Measurements section. For the second model this also involved including interactions between financial incentives and these control variables. Attitudes, self-efficacy, social influence, group training evaluation as well as reward responsiveness were included via means of the associated item scores in the analysis. We used the full conditional specification method (with the sequential regression procedure) to impute missing data. Several simulation studies suggest that this imputation method produces unbiased parameter estimates and standard errors ${ }^{34,35}$. The number of imputations was 55 and was set at least as large as the percentage of incomplete cases. ${ }^{36}$ As predictors for the multiple imputation we used all variables in the path analysis model. We also performed a complete case analysis including only participants with no missing data as a sensitivity analysis. In both the imputation and analysis phase (for available 
and complete cases), care was taken of possible clustering effects due to persons being nested within companies, by inclusion of a random intercept at the company level for each dependent variable in the model. Since medication use, NRT and abstinence at 12 months were binary variables, robust maximum likelihood was used, involving sandwich estimators of the standard errors of the regression coefficients.

\section{RESULTS}

\section{Loss to follow-up}

Of the 604 participants included in the CRT, 62 (9.9\%) did not fill out the follow-up questionnaire that was administered directly after finishing the smoking cessation program. The participants who did not complete the questionnaire directly after finishing the program had more often a low income level $(\leq € 1630)$ than participants who did complete the questionnaire $(58 \%$ versus $31 \%, p=0.001)$, had a higher mean nicotine dependence (Fagerström score 5.2 versus $4.3, p=0.004$ ), but did not depend significantly on education level, age, sex, and intervention condition.

\section{Participants}

Participant characteristics are presented in Table 1. Participants had a high reward responsiveness with a mean score of 3.6 for a maximum of 4 . Attitudes about quitting were positive on average, just like the perceived social norm toward quitting and social support. Self-efficacy of participants was high with a mean score of 4.5 out of 5.0. Participants graded the smoking cessation group training program with a high mark of 7.8 out of 10 . Of the total number of 604 participants in the study, 34\% (206/604) had a $\mathrm{CO}$ value $\leq 9 \mathrm{ppm}$ and were registered as continuously abstinent between the end of the smoking cessation program and 12 months later. In the intervention group with financial incentives the 12-month quit rate was $41 \%(131 / 319)$ and in the control group $26 \%(75 / 285)$. 
Table 1. Participant characteristics at baseline, after finishing the smoking cessation group training program and after 12 months $(\mathrm{n}=604)$

\begin{tabular}{|c|c|c|c|}
\hline Characteristic & Baseline & $\begin{array}{c}\text { After the smoking } \\
\text { cessation training } \\
\text { program }\end{array}$ & After 12 months \\
\hline Age $(n=599)$ & $45.1(10.2)$ & - & - \\
\hline $\begin{array}{l}\text { Sex }(n=604) \\
\text { Women } \\
\text { Men }\end{array}$ & $\begin{array}{l}223(36.9 \%) \\
381(63.1 \%)\end{array}$ & - & - \\
\hline $\begin{array}{l}\text { Educational level }(\mathrm{n}=579) \\
\text { Low } \\
\text { Moderate } \\
\text { High }\end{array}$ & $\begin{array}{l}159(27.5 \%) \\
255(44.0 \%) \\
165(28.5 \%)\end{array}$ & - & - \\
\hline $\begin{array}{l}\text { Income Level }(n=485) \\
\text { Low } \\
\text { Moderate } \\
\text { High }\end{array}$ & $\begin{array}{l}179(33.5 \%) \\
175(32.7 \%) \\
181(33.8 \%)\end{array}$ & - & - \\
\hline $\begin{array}{l}\text { Nicotine dependence (Fagerström score 1-10) } \\
(\mathrm{n}=573)\end{array}$ & $4.4(2.0)$ & - & - \\
\hline Reward responsiveness* $(n=580)$ & $3.6(0.4)$ & - & - \\
\hline $\begin{array}{l}\text { Financial incentives }(n=604) \\
\text { Yes } \\
\text { No }\end{array}$ & $\begin{array}{l}319(52.8 \%) \\
285(47.2 \%)\end{array}$ & - & - \\
\hline $\begin{array}{l}\text { Medication use }(n=536) \\
\text { Yes } \\
\text { No }\end{array}$ & - & $\begin{array}{l}120(22.4 \%) \\
416(77.6 \%)\end{array}$ & - \\
\hline $\begin{array}{l}\text { Nicotine replacement therapy }(n=537) \\
\text { Yes } \\
\text { No }\end{array}$ & - & $\begin{array}{l}264(49.2 \%) \\
273(50.8 \%)\end{array}$ & \\
\hline Attitudes about quitting (1-5) $(n=524)$ & - & $4.7(0.5)$ & - \\
\hline Self-efficacy for quitting (1-5) $(n=522)$ & - & $4.5(0.5)$ & - \\
\hline Social support for quitting $(1-3)(n=520)$ & - & $2.4(0.5)$ & - \\
\hline Subjective norm about quitting $(1-5)(n=523)$ & - & $4.7(0.5)$ & - \\
\hline Training program evaluation (1-5) $(n=517)$ & - & $3.9(0.7)$ & - \\
\hline Training program score $(1-10)(n=512)$ & - & $7.8(1.4)$ & - \\
\hline $\begin{array}{l}\text { Carbon monoxide scores of participants abstinen } \\
\text { at } 12 \text { months follow-up }(n=206) \\
\leq 4 \mathrm{ppm} \\
\leq 9 \mathrm{ppm}\end{array}$ & & & $\begin{array}{l}199(96.6 \%) \\
206(100.0 \%)\end{array}$ \\
\hline $\begin{array}{l}\text { Quit success }(n=603) \\
\text { Yes } \\
\text { No }\end{array}$ & - & - & $\begin{array}{l}206(34.2 \%) \\
397(65.8 \%)\end{array}$ \\
\hline
\end{tabular}

Note: numbers are Mean (SD) or $\mathrm{n}(\%)$

* BAS Reward Responsiveness Scale. A higher score on the scale (maximum 4) indicates a higher reward responsiveness. 


\section{Correlations}

Table 2 displays correlations between financial incentives, training program, medication use, NRT use, reward responsiveness, psychosocial mediators, and successful quitting smoking. Financial incentives correlated weakly with medication use, self-efficacy for quitting smoking and quit success. Training program evaluation was weakly to moderately correlated with each of the psychosocial mediators (attitudes, self-efficacy and social influence), and weakly correlated with medication use, NRT use, and quit success. Quit success was correlated most with self-efficacy for quitting and attitudes about quitting. Reward responsiveness was not significantly correlated with any of the variables.

Table 2. Pearson correlations between financial incentives, mediating variables, and smoking cessation.

\begin{tabular}{llllllllll}
\hline & $\mathbf{1}$ & $\mathbf{2}$ & $\mathbf{3}$ & $\mathbf{4}$ & $\mathbf{5}$ & $\mathbf{6}$ & $\mathbf{7}$ & $\mathbf{8}$ & $\mathbf{9}$ \\
\hline Financial incentives & 1 & & & & & & & & \\
Program evaluation & 0.07 & 1 & & & & & & & \\
Medication use & $0.11^{*}$ & $0.13^{* *}$ & 1 & & & & & & \\
Nicotine replacement use & -0.06 & $0.11^{*}$ & $0.34^{* * *}$ & 1 & & & & & \\
Reward responsiveness & 0.002 & 0.01 & -0.71 & 0.08 & 1 & & & & \\
Attitudes about quitting & 0.07 & $0.25^{* * *}$ & 0.02 & 0.06 & 0.02 & 1 & & & \\
Self-efficacy for quitting & $0.14^{* *}$ & $0.26^{* * *}$ & 0.09 & -0.06 & 0.05 & $0.38^{* * *}$ & 1 & & \\
Social influence on quitting & 0.10 & $0.30^{* * *}$ & 0.04 & 0.04 & -0.03 & $0.34^{* * *}$ & $0.19^{* * *}$ & 1 \\
Quit success & $0.15^{* * *}$ & $0.15^{* *}$ & $0.10^{*}$ & -0.08 & -0.05 & $0.19^{* * *}$ & $0.28^{* * *}$ & $0.14^{* *}$ & 1 \\
\hline
\end{tabular}

${ }^{*} \mathrm{p}<.05,{ }^{* *} \mathrm{p}<.01{ }^{* * *} \mathrm{p}<.001$

\section{Two-level path analysis}

Figure 1 presents the results of the path analysis. As supported by an average CFI of 0.999, an average TLI of 1.000 and an average RMSEA of 0.008 , the model fit was good. The model explained $15.2 \%$ of the variance in quit success.

The residual intraclass correlation coefficients (ICC) indicate that there are (small) clustering effects due to persons being nested within companies: ICC $=0.089$ for training program evaluation, $I C C=0.027$ for attitude, $I C C=0.011$ for self-efficacy, $I C C=0.051$ for social influence, ICC $=0.084$ for medication use, ICC $=0.068$ for NRT use, and ICC $=0.037$ for quit success.

The model shows that financial incentives were positively and directly associated with quit success $(\beta=0.16, p=0.001)$. Financial incentives were also associated with a higher self-efficacy for quitting smoking $(\beta=0.12, p=0.017)$ and with more use of smoking cessation medication ( $\beta=0.09, p=0.045$ ). Financial incentives did not significantly influence appraisal of the smoking cessation program, nor influenced attitudes or social influence on quitting smoking. A higher evaluation of the smoking cessation training program was associated with a more positive attitude about quitting smoking, higher self-efficacy for quitting smoking and positive social influence on quitting smoking. Of these three psychosocial mediators, self-efficacy $(\beta=0.19, p<0.001)$, as well as social influence $(\beta=0.13$, 
$p=0.042$ ) were associated with quit success. The complete case analysis ( $n=328)$ showed comparable mediation pathways with the analysis based on multiple imputation (see supplementary file 2). The complete case analysis did not show a significant association between financial incentives and medication use, nor an association between training program evaluation and attitude, which probably reflects a lack of statistical power due to the smaller number of participants involved in the analysis.

The associations of financial incentives with quit success and other variables in the model did not depend significantly on the participant's reward responsiveness nor on the participant's sex and age (all p-values $>0.15$ ). However, the effect of the intervention on social influence and the effect on self-efficacy were close to significance moderated by educational $(p=0.051)$ and income level $(p=0.076)$ respectively. In follow-up analyses exploring these interactions, a new mediating path from financial incentives via a higher social influence $(\beta=0.09, p=0.022)$ to a higher quit success was found for the subgroup of participants with a moderate education (but not for the low and high education groups). In addition, the association between financial incentives and self-efficacy was statistically significant for the high income group $(\beta=0.21, p=0.008)$ and for the moderate income group $(\beta=0.20, p=0.014)$, but was not significant for the low income group $(p=0.419)$. This implies that the mediating path from financial incentives via self-efficacy on quit success as previously found for the whole sample, apparently only holds for the high and moderate income groups. 


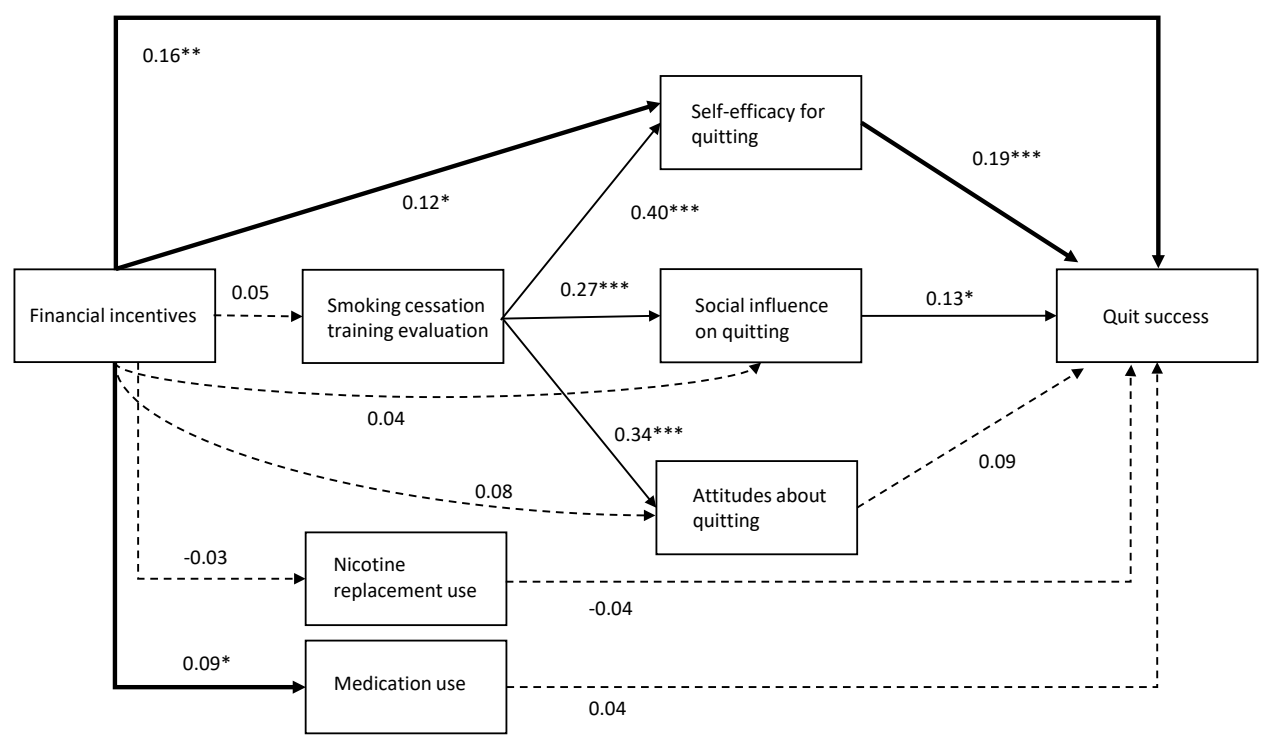

Figure 1. Path analysis model with unstandardized regression coefficients assessing the pathways between financial incentives and quit success. Available case analysis with multiple imputation ( $\mathrm{n}$ $=604$ ), and with random intercepts at company level. Solid arrows depict statistically significant pathways, dashed lines depict nonsignificant pathways. Thick arrows represent significant direct and mediational pathways from financial incentives to quit success. Only pathways of interest are shown. Control variables were omitted from the figure for simplification.

\section{DISCUSSION}

The current study provides insight into the pathways through which financial incentives can increase quit success in smokers. In our path analysis model, financial incentives were associated with a higher use of smoking cessation medication, and incentives were associated with quit success via an indirect pathway through increased self-efficacy, but not via the smoking cessation training program evaluation, attitudes, and social influence.

The results showed a pathway from financial incentives to an increased use of smoking cessation medication. This result may be explained by the idea that financial incentives increase the determination of a smoker to be successful in this particular quit attempt, which motivates the individual to use medication in order to enhance his/her chance of quit success and earn the incentives. If incentives dependent upon quit success can motivate smokers to use smoking cessation medication, which was also found in previous studies, ${ }^{16-18}$ it can be an important strategy to increase successful smoking cessation ${ }^{19}$ that should be further explored. This result also implies that if financial incentives are used to promote smoking cessation, making cessation medication easily accessible and freely available along with the incentives may increase the intervention's impact. ${ }^{11}$ Although the positive effect of smoking cessation medication use on quit success has been shown 
in previous research, ${ }^{19}$ the current study did not show a statistically significant pathway leading from medication use to quit success, likely due to a lack of statistical power.

The analysis also showed that a mediational pathway ran from financial incentives to quit success by an increased self-efficacy for quitting smoking. From previous research, it is known that there is a strong association between self-efficacy for quitting smoking and quit success, ${ }^{37,38}$ which is also reflected in the current model. However, this is the first study that has investigated the association between financial incentives and self-efficacy. A potential explanation of the association between the incentives and self-efficacy is that the incentives encourage people to visualize achieving their goal of quitting smoking and receiving the reward. This visualization of successfully performing a behavior (such as quitting smoking) can enhance a person's self-efficacy for that behavior. ${ }^{39}$ Because a high self-efficacy has shown to be a good predictor of successful behavioral change, ${ }^{37,38,40}$ financial incentives may be a novel way to increase self-efficacy and improve quit success which should be explored in further research. Another possibility for the association between the incentives and self-efficacy, is the self-efficacy-as-motivation argument, ${ }^{41}$ which states that because of the way that self-efficacy is measured, by asking how confident a person is to perform or resist a certain behavior, self-efficacy scores reflect motivation. ${ }^{41}$ This implies that the positive association in the current study between the incentives and self-efficacy actually represents an increase in the participants' motivation to quit smoking elicited by the financial incentives. Remarkably, the mediational pathway through self-efficacy only seemed to apply to the high and moderate income groups, but not the low income group. This finding should be replicated and explored further in future research.

The main results of the current study did not show significant pathways between financial incentives and quit success via attitudes and social influence. It is possible that because the average attitude toward quitting was already very positive in the current sample (mean score 4.7 out of 5.0), the incentives could not further increase this positive attitude. It should be further assessed whether in populations of smokers with less positive attitudes toward smoking cessation incentives could make a difference. The follow-up analyses did show a mediational pathway from financial incentives via a higher social influence to quit success, but only for the subgroup of participants with a moderate education level. Future research should explore whether this education-dependent association between financial incentives and social influence for quitting smoking can be replicated and whether and why it is dependent on educational level. Previous research suggests that financial incentives may be even more effective in stimulating support from the smoker's social environment if they are shared with significant others, for example as was done in a study with pregnant women ${ }^{23}$ where both the woman and a designated social supporter received financial incentives if the expectant mother quit smoking.

The direct effect of financial incentives that was revealed in the current model may indicate that incentives work separately from the established pathways leading to behavioral change. It is also possible that this pathway is mediated by other mediators like intention or action planning ${ }^{42}$ which we did not measure in the current study. 
A path that was not statistically significant in the current study, was the path leading from financial incentives via smoking cessation training and the psychosocial mediators to quit success. The absence of an association between the financial incentives and training appraisal may indicate that financial incentives do not affect how participants value a smoking cessation training program. Alternatively, the high appreciation of the training by the participants may have caused a ceiling effect. Nonetheless, the model indicated that the evaluation of the group smoking cessation training program was strongly associated with social influence, self-efficacy and attitudes. This result was not surprising, since the group training program included components aiming to increase these psychosocial mediators, and since group behavioral therapy has proven to be an effective method for smoking cessation. ${ }^{43}$ The results of the current study demonstrate an important contribution of the smoking cessation training program to predictors of quit success, and we therefore recommend that financial incentives should be used in combination with an effective behavioral intervention.

Contrary to our hypothesis, we did not find that individual reward responsiveness affected the association between financial incentives and quit success. It is possible that, because we advertised during the recruitment period that there was a chance to receive financial incentives, the participants who subscribed to our study mainly consisted of people with a high reward responsiveness, which could have resulted in selection bias. Yet, the mean scale score of 3.6 found in the current study is comparable to scores found in previous research ranging from 3.24 to $3.52^{30,44-46}$ and thus may not be particularly high. However, it may be possible that our participants all exceeded a certain threshold level of reward responsiveness, above which the incentives were not increasingly effective to stimulate quit success. Alternatively, the BAS Reward Responsiveness scale may measure a more general trait and might not be sensitive enough to measure differences in responsiveness to the financial incentives. There may be other effect modifiers, such as individual differences in impulsiveness or the preference for immediate versus delayed rewards (delay discounting), ${ }^{5}$ which could be explored in further research.

\section{Strengths and Limitations}

This is the first study that investigates the causal pathways leading from providing financial incentives for smoking cessation to quit success. Important strengths of this study are that the data are from a CRT, which increases confidence in the causal effect of financial incentives on quit success because of the randomization into intervention and control groups, and the biochemical validation of smoking abstinence. A limitation of the design of the current study is that it does not allow interpreting all associations as causal effects. In the current study, we used a cutoff value of 9 ppm based on West et al. (2005). ${ }^{31}$ While it has been suggested that a cutoff criterion of $\leq 4 \mathrm{ppm}$ is more sensitive to detect recent smoking, ${ }^{47}$ using this stricter criterion would not have changed our conclusions as only 7 of the 206 abstinent participants had a CO value above 4 ppm.

Another limitation of the current study is that we could not incorporate the psychosocial mediator 'intention to quit smoking', in the model, which according to behavioral change theories ${ }^{13,14,21}$ is predicted by attitudes, social influence and self-efficacy, and precedes behavior. The reason that we could not include intention to quit in the model, is that at the 
follow-up measurement directly after finishing the smoking cessation training program, our study population included both smokers and successful quitters, and only participants who had not successfully quit smoking were asked whether they intended to quit smoking in the (near) future. It is possible that by not being able to include intention to quit in the model, we have missed a mediator of the effect of financial incentives. Another issue is that the scale for measuring 'social influence' had a Cronbach's alpha of 0.62, which is below, what is considered to be an acceptable level of $0.70{ }^{48}$ However, the construct social influence was considered a formative construct in the current study, and therefore its internal consistency is of little importance. ${ }^{49}$ The scale for measuring 'attitude' had a Cronbach's alpha of 0.67 , also being below the acceptable level of 0.7 , which may have led to attenuation effects in the analysis. A factor that should be taken into consideration is that participants in the current study were probably intrinsically motivated to quit smoking, because they voluntarily signed up for an extensive smoking cessation program without the certainty of receiving financial incentives. Therefore, the paths found in the current study may not be generalizable to extrinsically motivated smokers.

\section{Conclusions}

This study provides insight in how financial incentives increase quit success, and our findings suggest that financial incentives may be used to increase medication use and self-efficacy for quitting smoking, which offers an indirect way to improve successful smoking cessation. 


\section{REFERENCES}

1. Higgins ST, Davis DR, Kurti AN. Financial incentives for reducing smoking and promoting other health-related behavior change in vulnerable populations. Policy Insights from the Behavioral and Brain Sciences. 2017;4(1):33-40.

2. Mitchell MS, Goodman JM, Alter DA, et al. Financial incentives for exercise adherence in adults: systematic review and meta-analysis. Am J Prev Med. 2013;45(5):658-667.

3. Notley C, Gentry S, Livingstone-Banks J, Bauld L, Perera R, Hartmann-Boyce J. Incentives for smoking cessation. Cochrane Database of Systematic Reviews. 2019(7).

4. Haff N, Patel MS, Lim R, et al. The Role of Behavioral Economic Incentive Design and Demographic Characteristics in Financial Incentive-Based Approaches to Changing Health Behaviors: A MetaAnalysis. Am J Health Promot. 2015;29(5):314-323.

5. Miglin R, Kable JW, Bowers ME, Ashare RL. Withdrawal-Related Changes in Delay Discounting Predict Short-Term Smoking Abstinence. Nicotine \& tobacco research : official journal of the Society for Research on Nicotine and Tobacco. 2017;19(6):694-702.

6. Gneezy U, Meier S, Rey-Biel P. When and why incentives (don't) work to modify behavior. The Journal of Economic Perspectives. 2011;25(4):191-209.

7. Mantzari E, Vogt F, Shemilt I, Wei Y, Higgins JP, Marteau TM. Personal financial incentives for changing habitual health-related behaviors: A systematic review and meta-analysis. Prev Med. 2015;75:75-85.

8. Giles EL, Robalino S, McColl E, Sniehotta FF, Adams J. The Effectiveness of Financial Incentives for Health Behaviour Change: Systematic Review and Meta-Analysis. PLoS One. 2014;9(3):e90347.

9. van den Brand FA, Nagelhout GE, Winkens B, Chavannes NH, van Schayck OCP. Effect of a workplace-based group training programme combined with financial incentives on smoking cessation: a cluster-randomised controlled trial. Lancet Public Health. 2018;3(11):e536-e544. doi:10.1016/S2468-2667(18)30185-3.

10. Cahill K, Hartmann-Boyce J, Perera R. Incentives for smoking cessation. Cochrane Database Syst Rev. 2015(5):Cd004307.

11. Van den Brand FA, Nagelhout GE, Reda AA, et al. Healthcare financing systems for increasing the use of tobacco dependence treatment. Cochrane Database Syst Rev. 2017(9).

12. van den Brand FA, Nagelhout GE, Winkens B, et al. The effect of financial incentives on top of behavioral support on quit rates in tobacco smoking employees: study protocol of a clusterrandomized trial. BMC Public Health. 2016;16(1):1056.

13. Vries HD, Mudde AN. Predicting stage transitions for smoking cessation applying the attitudesocial influence-efficacy model. Psychol Health. 1998;13(2):369-385.

14. Ajzen I. The theory of planned behavior. Organ Behav Hum Decis Process. 1991;50(2):179-211.

15. Kim A, Kamyab K, Zhu J, Volpp K. Why are Financial Incentives not Effective at Influencing Some Smokers to Quit? Results of a Process Evaluation of a Worksite Trial Assessing the Efficacy of Financial Incentives for Smoking Cessation. J Occup Environ Med. 2011;53(1):62-67.

16. Etter J-F, Schmid F. Effects of Large Financial Incentives for Long-Term Smoking CessationA Randomized Trial. J Am Coll Cardiol. 2016;68(8):777-785.

17. Fraser DL, Fiore MC, Kobinsky K, et al. A Randomized Trial of Incentives for Smoking Treatment in Medicaid Members. Am J Prev Med. 2017;53(6):754-763.

18. Mantzari E, Vogt F, Marteau TM. The effectiveness of financial incentives for smoking cessation during pregnancy: is it from being paid or from the extra aid? BMC Pregnancy Childbirth. 2012;12:24. 
19. Cahill K, Stevens S, Perera R, Lancaster T. Pharmacological interventions for smoking cessation: an overview and network meta-analysis. Cochrane Database Syst Rev. 2013(5):CD009329.

20. van den Putte B, Yzer MC, Brunsting S. Social influences on smoking cessation: a comparison of the effect of six social influence variables. Prev Med. 2005;41(1):186-193.

21. de Vries H, Mudde A, Leijs I, et al. The European Smoking Prevention Framework Approach (EFSA): an example of integral prevention. Health Educ Res. 2003;18(5):611-626.

22. Van den Brand FA, Dohmen LME, Van Schayck CP, Nagelhout GE. 'Secretly, it's a competition': a qualitative study investigating what helped employees quit smoking during a workplace smoking cessation group training programme with incentives. BMC Open. 2018;8(11).

23. Donatelle RJ, Prows SL, Champeau D, Hudson D. Randomised controlled trial using social support and financial incentives for high risk pregnant smokers: Significant Other Supporter (SOS) program. Tob Control. 2000;9.

24. Bandura A. Self-efficacy: toward a unifying theory of behavioral change. Psychol Rev. 1977;84(2):191-215.

25. Martin LE, Cox LS, Brooks WM, Savage CR. Winning and losing: differences in reward and punishment sensitivity between smokers and nonsmokers. Brain Behav. 2014;4(6):915-924.

26. Alexander AC, Hébert ET, Businelle MS, Kendzor DE. Greater perceived importance of earning abstinence-contingent incentives is associated with smoking cessation among socioeconomically disadvantaged adults. Addict Behav. 2019;95:202-205.

27. Nestor LJ, McCabe E, Jones J, Clancy L, Garavan H. Smokers and ex-smokers have shared differences in the neural substrates for potential monetary gains and losses. Addict Biol. 2016:n/a-n/a.

28. Heatherton TF, Kozlowski LT, Frecker RC, Fagerstrom K-O. The Fagerström Test for Nicotine Dependence: a revision of the Fagerstrom Tolerance Questionnaire. Br J Addict. 1991;86(9):11191127.

29. Hoving E, Mudde A, De Vries H. Smoking and the $\varnothing$ pattern; predictors of transitions through the stages of change. Health Educ Res. 2006;21(3):305-314.

30. Carver CS, White TL. Behavioral inhibition, behavioral activation, and affective responses to impending reward and punishment: The BIS/BAS Scales. J Pers Soc Psychol 1994;67(2):319.

31. West RPLJ. Outcome criteria in smoking cessation trials: proposal for a common standard. Addiction. 2005;100.

32. Hox JJ, Bechger TM. An introduction to structural equation modeling. 1998.

33. MacKinnon DP, Lockwood CM, Hoffman JM, West SG, Sheets V. A comparison of methods to test mediation and other intervening variable effects. Psychol Methods. 2002;7(1):83-104.

34. Raghunathan TE, Lepkowski JM, Van Hoewyk J, Solenberger P. A multivariate technique for multiply imputing missing values using a sequence of regression models. Surv Methodol. 2001;27(1):85-96.

35. Van Buuren S, Brand JP, Groothuis-Oudshoorn CG, Rubin DB. Fully conditional specification in multivariate imputation. J Stat Comput Simul. 2006;76(12):1049-1064.

36. White IR, Royston P, Wood AM. Multiple imputation using chained equations: issues and guidance for practice. Stat Med. 2011;30(4):377-399.

37. Nagelhout GE, de Vries H, Fong GT, et al. Pathways of change explaining the effect of smokefree legislation on smoking cessation in The Netherlands. An application of the international tobacco control conceptual model. Nicotine Tob Res. 2012;14(12):1474-1482.

38. Armitage CJ, Conner M. Efficacy of the Theory of Planned Behaviour: a meta-analytic review. $\mathrm{Br}$ J Soc Psychol. 2001;40(Pt 4):471-499. 
39. Bandura A. Regulation of cognitive processes through perceived self-efficacy. Dev Psychol. 1989;25(5):729.

40. Bandura A. Health Promotion by Social Cognitive Means. Health Educ Behav. 2004;31(2):143164.

41. Williams DM, Rhodes RE. The confounded self-efficacy construct: conceptual analysis and recommendations for future research. Health Psychol Rev. 2016;10(2):113-128.

42. Van Osch L, Reubsaet A, Lechner L, Candel M, Mercken L, De Vries H. Predicting parental sunscreen use: Disentangling the role of action planning in the intention-behavior relationship. Psychol Health. 2008;23(7):829-847.

43. Stead LF, Carrol AJ, Lancaster T. Group behaviour therapy programmes for smoking cessation Cochrane Database Syst Rev 2017.

44. Baumann MR, Oviatt D, Garza RT, et al. Variation in BAS-BIS profiles across categories of cigarette use. Addict Behav. 2014;39(10):1477-1483.

45. Cui Y, Robinson JD, Engelmann JM, et al. Reinforcement sensitivity underlying treatmentseeking smokers' affect, smoking reinforcement motives, and affective responses. Psychol Addict Behav. 2015;29(2):300-311.

46. Bailey SR, Bryson SW, Killen JD. Predicting successful 24-hr quit attempt in a smoking cessation intervention. Nicotine Tob Res. 2011;13(11):1092-1097.

47. Perkins KA, Karelitz JL, Jao NC. Optimal Carbon Monoxide Criteria to Confirm 24-hr Smoking Abstinence. Nicotine \& Tobacco Research. 2012;15(5):978-982.

48. Field A. Discovering statistics using IBM SPSS statistics. sage; 2013.

49. Diamantopoulos A, Winklhofer HM. Index Construction with Formative Indicators: An Alternative to Scale Development. Journal of Marketing Research. 2001;38(2):269-277. 


\section{Supplementary file 1: Questions to evaluate the smoking cessation training program}

The following statements are about the stop-smoking group training. Indicate to what extent you agree with the following statements:

\section{Response categories:}

Completely disagree (2) Disagree (3) Don't disagree/don't agree (4) Agree (5) Completely agree (6) Don't know

* Items $f$ and $g$ were reverse scored.
a. I found the program interesting
$\begin{array}{llllll}1 & 2 & 3 & 4 & 5 & 6\end{array}$
b. I found the program educational
$\begin{array}{llllll}1 & 2 & 3 & 4 & 5 & 6\end{array}$
c. I found the program clear
$\begin{array}{llllll}1 & 2 & 3 & 4 & 5 & 6\end{array}$
d. I liked the program
$\begin{array}{llllll}1 & 2 & 3 & 4 & 5 & 6\end{array}$

e. I have received sufficient information about quitting smoking

f. Participating in the program took me a lot of effort

$\begin{array}{llllll}1 & 2 & 3 & 4 & 5 & 6 \\ 1 & 2 & 3 & 4 & 5 & 6\end{array}$

h. I thought the total duration of the program was good

$\begin{array}{llllll}1 & 2 & 3 & 4 & 5 & 6\end{array}$

i. The program has met my needs

$\begin{array}{llllll}1 & 2 & 3 & 4 & 5 & 6\end{array}$

j. I will recommend participating in the training if someone $\begin{array}{lllllll}1 & 2 & 3 & 4 & 5 & 6\end{array}$ asks me to do so
k. I am satisfied with the quality of the program
I. I am satisfied with the type of help I have received $\begin{array}{lllllll}1 & 2 & 3 & 4 & 5 & 6\end{array}$ through the program
$\mathrm{m}$. Communication between my coach and me during the $\begin{array}{lllllll}1 & 2 & 3 & 4 & 5 & 6\end{array}$ program went well

What rating would you give the stop-smoking group training?
1
2
3
4
5
6
7
8
9
10 


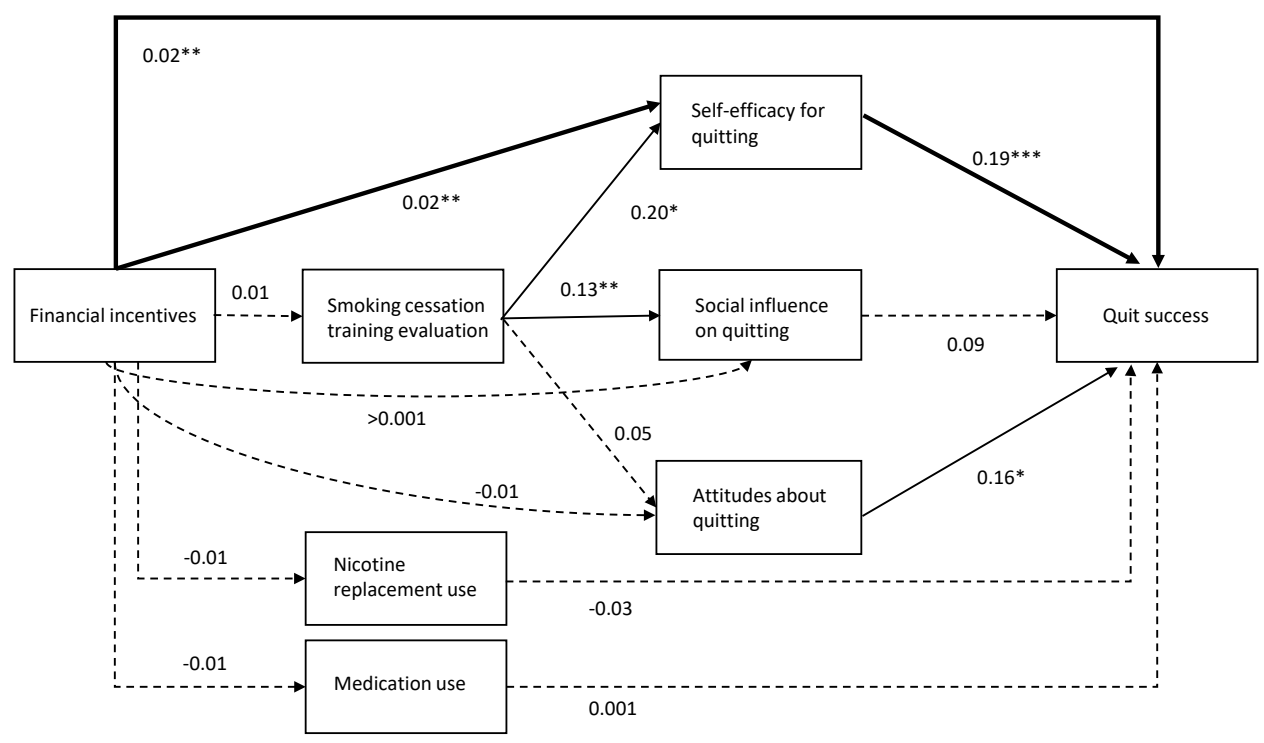

Supplementary file 2. Path analysis model with unstandardized regression coefficients assessing the pathways between financial incentives and quit success.

Complete case analysis $(n=328)$ with random intercepts at company level. Solid arrows depict statistically significant pathways, dashed lines depict non-significant pathways. Thick arrows represent significant direct and mediational pathways from financial incentives to quit success. Only pathways of interest are shown. Control variables were omitted from the figure for simplification. 

CHAPTER 9

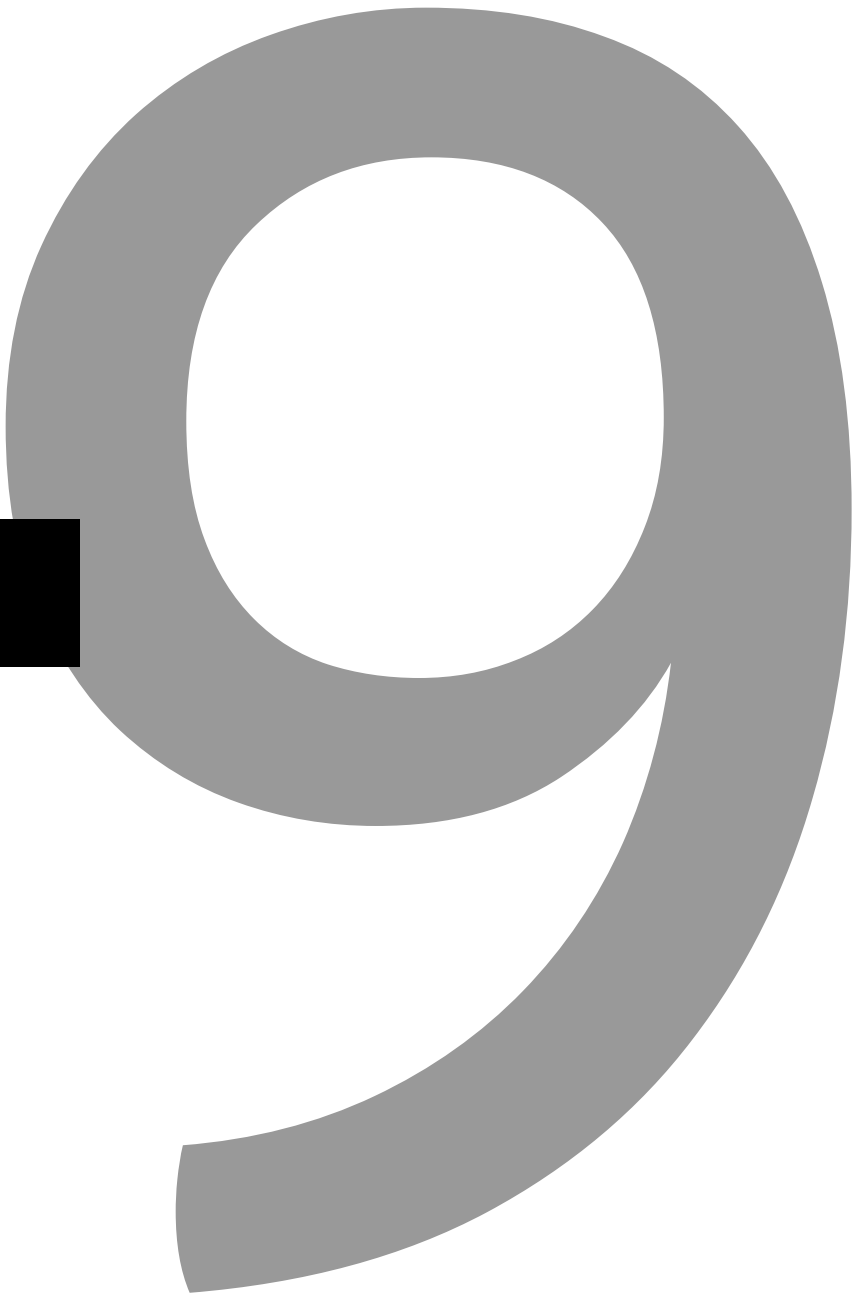




\section{Cost-effectiveness and cost-utility analysis of a workplace smoking cessation intervention with and without financial incentives}

Van den Brand FA, Nagelhout GE, Winkens B, Chavannes NH, van Schayck OCP, Evers SMAA. Cost-effectiveness and cost-utility analysis of a work-place smoking cessation intervention with and without financial incentives 


\section{ABSTRACT}

Aims To perform an economic evaluation of a workplace smoking cessation group training program with incentives compared to a training program without incentives.

Design A trial-based cost-effectiveness analysis (CEA) and cost-utility analysis (CUA) from a societal perspective and from an employer's perspective.

Setting 61 companies in the Netherlands.

Participants 604 tobacco-smoking employees.

Intervention A 7-week workplace smoking cessation group training program. The intervention group earned gift vouchers of $€ 350$ for 12-month continuous abstinence.

Measurements Online questionnaires were administered to assess quality of life (EQ-5D$5 \mathrm{~L}$ ), and resource use during the 14-month follow-up period (2-month training period plus 12-month abstinence period). For the CEA the primary outcome measure was carbon monoxide (CO)-validated continuous abstinence; for the CUA the primary outcome was Quality Adjusted Life Years (QALY). Bootstrapping and sensitivity analyses were performed to account for uncertainty. Incremental cost-effectiveness ratio (ICER) tables were used to determine cost-effectiveness from a lifetime perspective.

Findings $O$ f the participants in the intervention group $41 \%$ had quit smoking compared to $26 \%$ in the control group. From a societal perspective with a 14-month follow-up period, the ICER per quitter for an intervention with financial incentives compared to no incentives was $€ 11,546$. From an employer's perspective, the ICER was $€ 5,686$. There was no significant difference in QALYs between the intervention and control group within the 14-month follow-up period. The intervention was dominated by the comparator in the primary analysis at a threshold of $€ 20,000$ euro per QALY. In the sensitivity analysis these results were surrounded with much uncertainty. A lifetime perspective showed an ICER of $€ 1249(95 \% \mathrm{Cl}=€ 850-€ 2,387)$ per QALY.

Conclusions Financial incentives compared to no financial incentives may be costeffective in increasing quitting smoking, particularly from a lifetime perspective. The results of the cost-utility analysis within the 14-month time frame were inconclusive, likely due to the relatively short observation period. 


\section{INTRODUCTION}

Smoking is the most preventable cause of illness and premature death, and smokingrelated illnesses lead to large health care costs and loss of productivity. ${ }^{1-6} \mathrm{~A}$ meta-analysis focusing on working populations found that smoking was associated with a $31 \%$ increase in risk of absenteeism and 2.9 more sickness absence days per year compared to nonsmoking. ' While many different types of interventions have been used to decrease smoking in society, ${ }^{8-11}$ stimulating individual smokers to quit still proves to be difficult, especially among smokers from lower socioeconomic groups. ${ }^{12-14}$

Smoking cessation interventions at the workplace have shown to be an effective approach to reach smokers and stimulate quitting smoking. ${ }^{11}$ Successful interventions in a workplace setting are group smoking cessation counselling and financial incentives for quit success. ${ }^{11,15-17}$ Stimulating smoking cessation at the workplace has benefits for both the employee and the employer. For the employee, apart from health benefits, smoking cessation counselling at the workplace is convenient, does not require a personal financial investment, and offers peer support from colleagues. ${ }^{11,18-20}$ For employers, investing in smoking cessation leads to healthier employees and can reduce costs that are associated with smoking, such as absenteeism ${ }^{4,21}$ and reduced performance, ${ }^{22,23}$ which were estimated to be $\$ 5,816$ per smoker per year. ${ }^{24}$

Inspired by these results, we designed a cluster-randomized controlled trial to investigate the effectiveness of financial incentives for smoking cessation, which was conducted within Dutch companies. Participants of this trial were adult employees who smoked, and who voluntarily signed up for a smoking cessation group training program at the workplace. Employees in companies that were randomized into the intervention condition additionally earned financial incentives totaling $€ 350$ if they quit smoking for 12 months. ${ }^{25}$ The results of this trial showed that significantly more participants had quit smoking in the intervention group with incentives $(41.1 \%$ vs. $26.4 \%, p<0.001)$ than in the control group with the cessation program alone. ${ }^{17}$

Apart from establishing the effectiveness of financial incentives for smoking cessation at the workplace, it is important to evaluate the broader costs and benefits of such an intervention, and to assess its cost-effectiveness from a societal perspective as well as from an employer's viewpoint. An economic evaluation of the costs and benefits of using financial incentives at the workplace to reduce smoking can improve the acceptability of incentives to society ${ }^{26}$ and could convince employers to offer a smoking cessation program with incentives to their employees. ${ }^{27}$

However, evidence on the cost-effectiveness of incentives for smoking cessation is sparse. To our knowledge, no study has yet performed a full economic evaluation of financial incentives alongside a trial with a population of employees who smoke. Additionally, no economic evaluation from an employer's viewpoint has been performed in this research field. One study that involved financial incentives worth $£ 400$ for smoking cessation during pregnancy found an incremental cost per quitter of $£ 1127$ (US $\$ 1716$ ) compared to routine care, but measured only costs directly related to the smoking cessation treatment 
and the incentives, but no other health care consumption nor did the study assess quality of life during the trial. ${ }^{28}$ However, the benefits of quitting smoking expressed in QALYS were estimated using a Markov model, and the authors estimated incremental costs of $£ 482(€ 541)$ per QALY. ${ }^{28}$ Another study that performed a cost-benefit analysis for a large cohort of Medicaid enrollees found that providing quitline callers with modest financial incentives was a cost-effective method to increase cessation rates over a 10-year horizon with a benefit-cost ratio of $1.30 .^{29}$

Research has shown that health improvements among employees who quit smoking can substantially reduce employers' costs related to productivity losses and absenteeism, and can decrease societal costs as a whole. ${ }^{6,21,30}$ When financial incentives are added to a smoking cessation program, at first they will inevitably increase the costs of the intervention. But if adding a reward to a smoking cessation program increases the number of quitters, the cost-effectiveness of the program could be improved, making financial incentives a short-term investment that pays off in the future.

The aim of the present study is to assess from a societal and an employer's perspective the cost-effectiveness (CEA) and cost-utility (CUA) of a workplace smoking cessation group training program with incentives compared to a training program without incentives in Dutch employees who smoke. It is hypothesized that providing financial incentives for quit success is associated with improved effects and health-related quality of life and decreased costs.

\section{METHODS}

\section{Reporting guidelines}

This study follows the Consolidated Health Economic Evaluation Reporting Standards (CHEERS) Statement. ${ }^{31}$

\section{Design}

A trial-based economic evaluation was performed alongside a cluster randomized controlled trial (RCT), which design has previously been described in detail in another publication..$^{25}$ The RCT was conducted within 61 companies in the Netherlands. Employees in both the intervention group and the control group participated in a smoking cessation training organized at the workplace, provided by the Dutch company SineFuma. SineFuma's smoking cessation program is registered in the Dutch Stop Smoking Quality Register for proven effective interventions. The training consisted of seven sessions of 90 minutes each, distributed over a period of two months. As part of the smoking cessation training, participants were informed about smoking cessation medication and encouraged to consider using medication during their quit attempt. Employees were recruited by the companies themselves. The aim of the RCT was to organize a single group training within each company and it was not intended to reach all smokers within a company. Participants in the intervention group additionally earned gift vouchers with a total amount of 350 euros if they quit smoking. Participants received via email a digital voucher which was sent by a research assistant if they were abstinent from smoking 
directly after finishing the smoking cessation program (€50), after 3 months $(€ 50)$ and after 6 months ( $€ 50)$; the last voucher of $€ 200$ was earned 12 months after finishing the program. The main outcome was successful quitting of smoking, defined as CO-validated and continuous smoking abstinence, from the moment that the participant had finished the training program up to 12 months later. Companies and participants were enrolled between March 2016 and March 2017.

\section{Participants}

All participants who had participated in the RCT were included in the current study Participants were male and female employees who were current tobacco smokers at the start of the trial, at least 18 years old, and who did not have a life-threatening disease at the start of the trial.

\section{Identification of costs}

The relevant costs that were identified were: intervention costs, health care costs, patient and family costs, and costs in other sectors. ${ }^{32,33}$ Intervention costs consisted of costs of the smoking cessation group training, costs of the incentives, and time costs. Health care costs use included visits to health care providers, costs of medical treatments, costs of overnight stay in hospitals, costs of home care, and costs of (smoking cessation) medications. Participant and family costs included travel costs for visits to health care services, and cost representing time spent by family members providing informal care (such as domestic work or taking care of the children). Costs in other sectors consisted of costs related to productivity, and will be referred to as 'productivity costs' in the remainder of this paper. Productivity costs included absenteeism from work, costs related to presenteeism, and costs for work breaks.

\section{Measurement and valuation of costs}

To measure all relevant resource use costs, a questionnaire was designed for this study, based on existing questionnaires. ${ }^{34-37}$ Participants were asked to report the data from their resource use questionnaire relating to the previous 3 months at baseline, and at 2 , 5, 8 and 14 months follow-up, with a recall period of 2, 3, 3 and 6 months, respectively. Online questionnaires were sent by email, and if participants preferred a paper version of the questionnaire, it was sent to them by post. Multiple email reminders were sent, and participants were called by phone to remind them to complete the questionnaire. Participants were awarded a gift voucher of 25 euros if they had completed the final questionnaire. The valuation of costs was based on Dutch guidelines. ${ }^{32,38}$ Cost prices were expressed in 2017 euros, based on the consumer price index (www.statline.cbs.nl). Due to the short time horizon of 14 months, costs were not discounted. Health care costs were estimated using a bottom-up (or micro-costing) approach. A detailed description of the cost valuation is presented in Supplementary file 1.

\section{Effects}

Smoking abstinence was measured by self-report and validated using expired air CO measurement. The research assistant visited the participants who claimed to be abstinent from smoking at the workplace to perform the CO measurement, or offered to travel to an alternative location that was suggested by the participant. If $\mathrm{CO}$ measurements were 
higher than the cut-off point of 9 parts per million, or if $\mathrm{CO}$ measurement could not be performed, participants were considered to be smokers (Russel's standard)..$^{39}$ The primary outcome for the CEA was cost per CO-validated continuously abstinent participant (cost per successful quitter). The primary outcome of the CUA was cost per Quality Adjusted Life Year (QALYs). The utility value derived from the standard health-related quality of life questionnaire, EuroQol-5D-5L ${ }^{40}$, using Dutch tariff ${ }^{41}$ was used to compute QALYs. The utilities at the various time points were used to compute QALYs over the 14-month time horizon by means of the area under the curve method, where the utility of a particular health state is multiplied by the time in this state. ${ }^{33,42}$ Costs (the use of resources) were measured continuously; outcomes for the economic evaluation study were measured at baseline (T0), directly after finishing the smoking cessation training program (two months after baseline, T1), and 5 months (T2), 8 months (T3), and 14 months (T4) after baseline.

\section{Statistical Analyses}

The analyses of the effect measure continuous abstinence from smoking were performed according to an intention to treat (ITT) protocol, where all withdrawals were considered to be smokers. For the cost analysis, only participants who provided cost data for at least one follow-up measurement point (T1-T4) were included. ${ }^{43}$ Data were analyzed using IBM SPSS Statistics version 25 and Microsoft Excel 2010. Differences between participants lost to follow up were tested using independent t-tests and Chi square tests. Two-sided $p$-values $\leq 0.05$ were considered statistically significant. Individual mean imputation was used to impute missing cost data. ${ }^{43}$ Mean costs and $95 \%$ confidence intervals, as well as baseline differences in costs and utilities between the intervention and control group were assessed using bootstrapping (1,000 replications). The incremental cost-effectiveness ratio (ICER) was calculated by dividing the incremental costs by the incremental effects/ QALYs. Bootstrap simulations (5,000 replications) were conducted in order to quantify the uncertainty around the ICER. ${ }^{44}$ The bootstrapped cost-effectiveness ratios were subsequently plotted on a cost-effectiveness plane, which is divided into four quadrants by a vertical line that reflects the difference in costs and the horizontal line reflects the difference in effectiveness. The choice of treatment depends on the willingness to pay (WTP), which is the maximum amount of money that society is prepared to pay for a gain in effectiveness. Based on a report of the Dutch National Health Institute, a WTP of $€ 20,000$ for an additional QALY was used in this study. ${ }^{45}$ There are no guidelines on the WTP for an additional quitter. The bootstrapped ICERs were also depicted in a costeffectiveness acceptability curve (CEAC) showing the probability that the use of incentives for smoking cessation in a business setting is cost-effective over a range of ceiling ratios. Additionally, to demonstrate the robustness of the base case findings, sensitivity analyses were performed. A sensitivity analysis was performed where respondents with extremely high total costs (based on the $95^{\text {th }}$ percentile) were excluded, a complete case analysis was done where only participants who had completed all follow-up questionnaires were included, an analysis from a societal perspective was done where self-reported smoking abstinence was used as outcome measure instead of CO-validated smoking abstinence, and an analysis was performed where only intervention costs were included. 


\section{Lifetime perspective analysis}

To provide an additional estimation of the cost-effectiveness of the current intervention from a lifetime perspective, we used the current intervention's cost and effect data as input, and used standardized tables from Stapleton \& West (2012) to determine the ICER. ${ }^{46}$ The tables offer estimates of life years gained by quitting smoking attributable to interventions with different effect sizes. The life years gained are adjusted for future lifetime cessation (proportion of smokers who are expected to quit smoking in their lifetime without an intervention: $2.5 \%$ per annum) and are adjusted for relapse after the final follow-up (12 month follow-up). The ICER was corrected for the proportion of study participants within the age groups $<35$ years, $35-54$ years, and $>54$ years. A discount rate of $3.5 \%$ was used. The results in life years can also be interpreted as QALYs, where the years are weighted according to the quality of life that is experienced during these years. ${ }^{46}$ Because there are no verified utility weights for ex-smokers and smokers for the Dutch population, we followed the recommendations of Stapleton et al. (2012), and assumed that 1 life year was equal to 1 QALY. ${ }^{46}$ We contacted the authors of Stapleton \& West (2012) and they checked our calculations.

\section{RESULTS}

\section{Loss to follow-up}

All 604 employees within 61 companies who had participated in the RCT were included in the current study (Figure 1). 406 participants (67\%) completed all five cost questionnaires including baseline measurement (T0 to T4). 41 participants (7\%) did not provide cost data in any of the follow-up questionnaires (T1 to T4). Within these 41 participants, there were 10 individuals of whom cost data of follow-up questionnaires were missing because they completed a shortened version of the questionnaire. After imputing missing values, cost data were available for 563 participants (93\%). Validated smoking cessation data was available for 603 employees; 1 employee was excluded from the analysis due to unavoidable loss to follow up according to Russell's Standard. ${ }^{47}$ The participants who did not provide any cost data were more likely to be in the intervention group (9.4\% vs. 3.9\%, $\mathrm{p}=0.007$ ), had a higher mean Fagerström score (5.5 vs. 4.3, $\mathrm{p}>0.001)$, had on average more pack years (mean 29.2 vs. 22.1, $p=0.046$ ), were more often low educated ( $45.7 \%$ vs. $26.3 \%$, $\mathrm{p}=0.037)$, and had more often a lower income $(66.7 \%$ vs. $31.5 \%, p<0.001)$, but did not differ significantly in age or sex. 


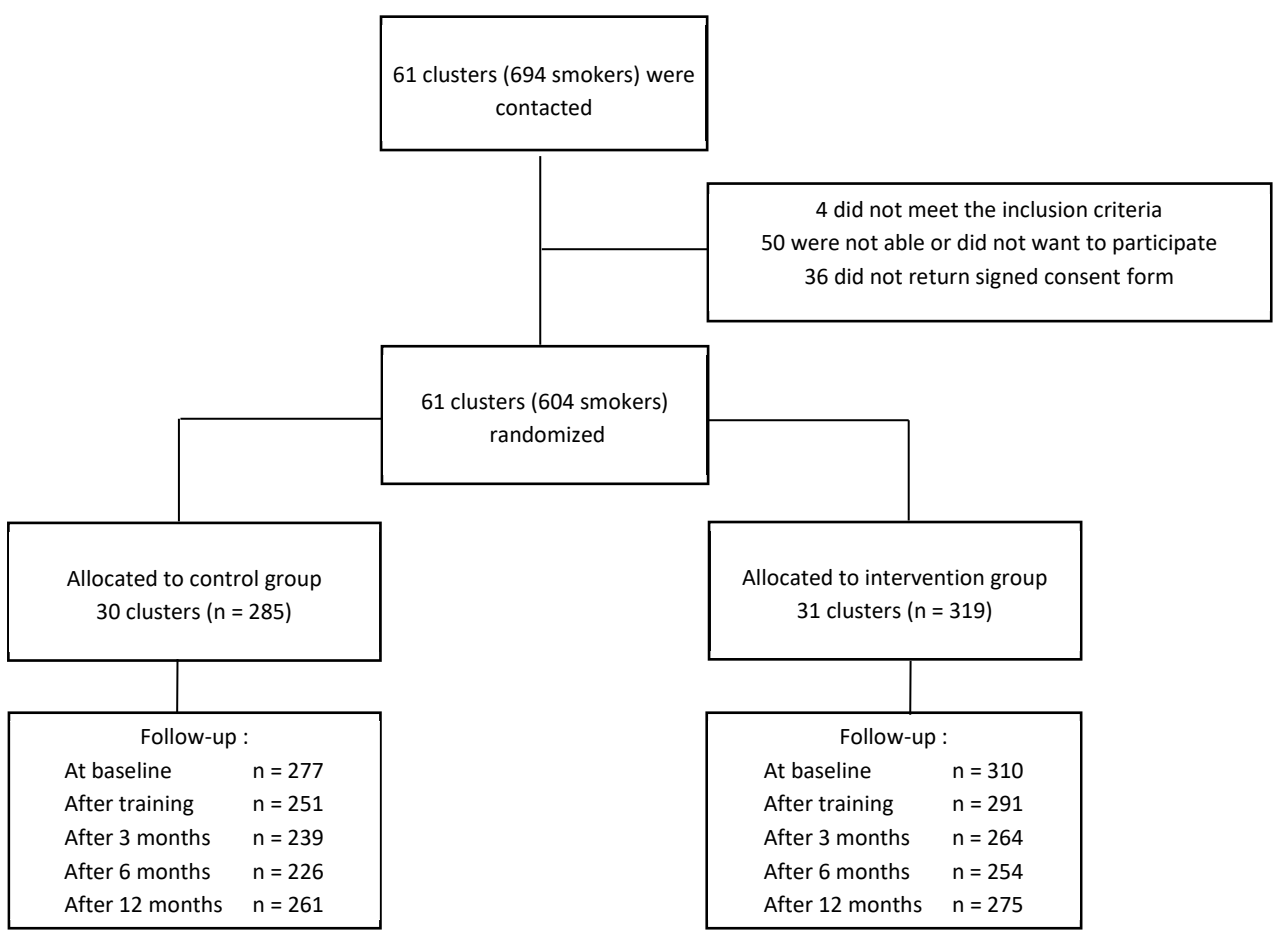

Figure 1. Flow-diagram of the study showing number of participants ( $n$ ) who provided cost data at each measuring point.

\section{Participant characteristics}

Participant characteristics and mean costs three months prior to baseline are presented in Table 1. There were no baseline differences between groups for total overall costs. Total health care costs were higher in the control group $(p=0.03)$; there were no differences between groups for patient and family costs or productivity costs.

\section{Costs and effects}

Mean 14-month resource use is displayed in Table 2. The largest costs for both groups were the costs related to productivity in general, and within this subcategory the costs of work breaks were the highest. Mean total health care costs, participant and family costs, and productivity costs did not differ significantly between intervention and control groups. The cost of the subcategory absenteeism was significantly higher in the intervention group than in the control group. The effect analyses showed that significantly more participants quit smoking in the intervention group $(131 / 319=41.1 \%)$ than in the control group $(75 / 284=26.4 \%)$. Of the 206 participants who were validated abstinent from smoking, 205 (99.5\%) had a CO score < 6 ppm at the final follow-up measurement (the single remaining participant had a CO score of 9). The 14-month QALY scores were not significantly different between the intervention and the control group. Comparing 14-month QALY scores between participants who were continuously abstinent $(\mathrm{QALY}=1.071, \mathrm{SD}=0.125)$ to unsuccessful quitters $(\mathrm{QALY}=1.057, \mathrm{SD}=0.153)$ showed no 
significant difference $(t(560)=1.14, p=0.256)$. Total volumes and costs over the entire 14-month follow-up period are presented in Table S1.

\section{4-month cost-effectiveness analyses}

The intervention condition with incentives resulted in higher costs and in higher effects compared to the control group (Table 3). The incremental costs or costs for an additional quitter from a societal perspective were $€ 11,546$. The cost-effectiveness plane for the main analysis from the societal perspective shows that most (92\%) of the bootstrapping results lie in the north-east quadrant (Figure 2a). The corresponding cost-effectiveness acceptability curve shows that the probability that the intervention is cost-effective is $80 \%$ (at $€ 20,000$ per abstinent smoker), up to $97 \%$ (at $€ 40,000$ per abstinent smoker). The sensitivity analysis from a societal perspective where participants with the highest costs (above the $95^{\text {th }}$ percentile) were removed showed an ICER of $€ 3,432$, and the complete case analysis showed an ICER of $€ 17,610$. The CEA from an employer's perspective showed ICERs varying between $€ 1,124$ and $€ 12,117$ (see Table 4 for all sensitivity analyses). The bootstrapped results are mainly presented in the north-east quadrant of the costeffectiveness plane (Figure $2 b$ ). This indicates that the intervention was more effective yet more costly, but also reveals that there was a large uncertainty surrounding the ICER. 
Table 1. baseline participant characteristics of control group and intervention group and health care costs and absenteeism costs of three months prior to baseline $(n=604)$.

\begin{tabular}{|c|c|c|}
\hline Variable & $\begin{array}{l}\text { Intervention group } \\
(\mathrm{n}=\mathbf{3 1 9})\end{array}$ & $\begin{array}{c}\text { Control group } \\
(n=285)\end{array}$ \\
\hline Age, mean (SD) & $43.9(10.4)$ & $46.6(9.7)$ \\
\hline $\begin{array}{l}\text { Gender, n (\%) } \\
\text { Women } \\
\text { Men }\end{array}$ & $\begin{array}{l}102(32.0) \\
217(68.0)\end{array}$ & $\begin{array}{l}121(42.5) \\
164(57.5)\end{array}$ \\
\hline $\begin{array}{l}\text { Educational level, n (\%) } \\
\text { Low } \\
\text { Moderate } \\
\text { High } \\
\text { Missing }\end{array}$ & $\begin{array}{l}97(30.4) \\
136(42.6) \\
75(23.5) \\
11(3.4)\end{array}$ & $\begin{array}{c}62(21.8) \\
119(41.8) \\
90(31.6) \\
14(4.9)\end{array}$ \\
\hline $\begin{array}{l}\text { Income level, n (\%) } \\
\text { Low } \\
\text { Moderate } \\
\text { High } \\
\text { Missing }\end{array}$ & $\begin{array}{l}111(34.8) \\
91(28.5) \\
76(23.8) \\
41(12.9)\end{array}$ & $\begin{array}{c}68(23.9) \\
84(29.5) \\
105(36.8) \\
28(12.5)\end{array}$ \\
\hline FTND $^{a}$ (range 0-10), mean (SD) & $4.4(1.9)$ & $4.5(2.0)$ \\
\hline Pack years, mean (SD) & $21.6(13.2)$ & $23.5(13.0)$ \\
\hline $\mathrm{QALY}{ }^{\mathrm{b}}$, mean $(\mathrm{SD})$ & $0.90(0.13)$ & $0.91(0.13)$ \\
\hline $\begin{array}{l}\text { Health care costs }(€) \text {, mean (SD) } \\
\text { General practitioner } \\
\text { Occupational doctor } \\
\text { Hospital } \\
\text { Other care } \\
\text { Home care } \\
\text { Medication } \\
\text { Smoking cessation medication } \\
\text { Smoking cessation coach } \\
\text { Total health care costs }\end{array}$ & $\begin{array}{c}21(35) \\
10(43) \\
76(334) \\
54(103) \\
0(4) \\
21(81) \\
1(13) \\
7(19) \\
189(399)\end{array}$ & $\begin{array}{c}22(41) \\
11(42) \\
134(624) \\
72(148) \\
4(63) \\
73(528) \\
1(6) \\
5(17) \\
318(912)\end{array}$ \\
\hline $\begin{array}{l}\text { Participant and family costs } \\
\text { Travel and parking costs } \\
\text { Informal care } \\
\text { Total participant and family costs }\end{array}$ & $\begin{array}{c}13(15) \\
115(475) \\
127(477)\end{array}$ & $\begin{array}{c}15(21) \\
61(236) \\
76(246)\end{array}$ \\
\hline $\begin{array}{l}\text { Productivity costs }(€) \text {, mean (SD) } \\
\text { Absenteeism } \\
\text { Presenteeism } \\
\text { Work breaks } \\
\text { Total productivity costs }\end{array}$ & $\begin{array}{l}456(1320) \\
289(1198) \\
1655(848) \\
2356(2137)\end{array}$ & $\begin{array}{l}364(1061) \\
210(674) \\
1640(913) \\
2165(1647)\end{array}$ \\
\hline Total costs & $2623(2534)$ & 2551 (2096) \\
\hline
\end{tabular}

${ }^{\text {a }}$ Fagerström Test for Nicotine Dependence 
Table 2. Mean 14-month costs of intervention and control group (1000 bootstrap replications).

\begin{tabular}{|c|c|c|c|}
\hline \multirow{2}{*}{$\begin{array}{l}\text { Cost type } \\
\text { Societal perspective }\end{array}$} & \multicolumn{2}{|c|}{ Costs per group $(€)$, mean (SD) } & \multirow{2}{*}{$\begin{array}{c}\begin{array}{c}\text { Difference } \\
(95 \% \mathrm{Cl})\end{array} \\
\begin{array}{l}\text { Intervention - } \\
\text { control group }\end{array}\end{array}$} \\
\hline & $\begin{array}{l}\text { Intervention } \\
\text { group }\end{array}$ & $\begin{array}{l}\text { Control } \\
\text { group }\end{array}$ & \\
\hline $\begin{array}{l}\text { Intervention costs }(n=319 / 285) \\
\text { Incentives } \\
\text { Smoking cessation group training } \\
\text { Time attending training (within working hours) } \\
\text { Total intervention costs }\end{array}$ & $\begin{array}{c}189(9) \\
421 \\
389 \\
998(9)\end{array}$ & $\begin{array}{r}- \\
421 \\
389 \\
809\end{array}$ & $\begin{array}{c}189 \text { (171 to } 206) \\
0 \\
0 \\
189(173 \text { to } 205)\end{array}$ \\
\hline $\begin{array}{l}\text { Health care costs }(n=289 / 274) \\
\text { General practitioner } \\
\text { Occupational doctor } \\
\text { Hospital }^{\mathrm{a}} \\
\text { Other care }^{\mathrm{b}} \\
\text { Home care } \\
\text { Medication } \\
\text { Smoking cessation medication } \\
\text { Smoking cessation coach } \\
\text { Total health care costs }\end{array}$ & $\begin{array}{c}103(8) \\
95(15) \\
919(200) \\
353(40) \\
175(144) \\
158(61) \\
76(9) \\
59(6) \\
1942(318)\end{array}$ & $\begin{array}{c}89(7) \\
49(9) \\
600(93) \\
282(26) \\
15(13) \\
268(101) \\
50(10) \\
72(6) \\
1423(167)\end{array}$ & $\begin{array}{c}14(-5 \text { to } 36) \\
45(12 \text { to } 78) \\
319(-67 \text { to } 781) \\
71(-19 \text { to } 166) \\
160(-26 \text { to } 474) \\
-110(-381 \text { to } 97) \\
26(-2 \text { to } 52) \\
-13(-32 \text { to } 4) \\
519(-122 \text { to } 1274)\end{array}$ \\
\hline $\begin{array}{l}\text { Participant and family costs }(n=289 / 274) \\
\text { Travel and parking costs } \\
\text { Informal care } \\
\text { Total patient and family costs }\end{array}$ & $\begin{array}{c}62(5) \\
761(122) \\
833(122)\end{array}$ & $\begin{array}{c}58(4) \\
484(137) \\
557(141)\end{array}$ & $\begin{array}{c}5(-7 \text { to } 17) \\
278(-119 \text { to } 644) \\
276(-103 \text { to } 615)\end{array}$ \\
\hline $\begin{array}{l}\text { Productivity costs }(n=289 / 274) \\
\text { Absenteeism } \\
\text { Presenteeism } \\
\text { Work breaks } \\
\text { Total productivity costs }\end{array}$ & $\begin{array}{l}3436(370) \\
1486(207) \\
7135(195) \\
12079(529) \\
\end{array}$ & $\begin{array}{l}2458(292) \\
1292(211) \\
7318(227) \\
11091(486)\end{array}$ & $\begin{array}{l}978(52 \text { to } 1932) \\
194(-387 \text { to } 823) \\
-183(-747 \text { to } 403) \\
988(-427 \text { to } 2417)\end{array}$ \\
\hline Employer's perspective & & & \\
\hline $\begin{array}{l}\text { Intervention costs }(n=319 / 285) \\
\text { Incentives } \\
\text { Smoking cessation group training } \\
\text { Time attending training (within working hours) } \\
\text { Total intervention costs }\end{array}$ & $\begin{array}{c}189(9) \\
421 \\
389 \\
998(9)\end{array}$ & $\begin{array}{c}- \\
421 \\
389 \\
809\end{array}$ & $\begin{array}{c}189 \text { (171 to } 206) \\
0 \\
0 \\
189(173 \text { to } 205)\end{array}$ \\
\hline $\begin{array}{l}\text { Productivity costs }(n=289 / 274) \\
\text { Absenteeism } \\
\text { Presenteeism } \\
\text { Work breaks } \\
\text { Total productivity costs }\end{array}$ & $\begin{array}{c}3436(370) \\
1486(207) \\
7135(195) \\
12079(529)\end{array}$ & $\begin{array}{c}2458(292) \\
1292(211) \\
7318(227) \\
11091(486)\end{array}$ & $\begin{array}{c}978(52 \text { to } 1932) \\
194(-387 \text { to } 823) \\
-183(-747 \text { to } 403) \\
988(-427 \text { to } 2417)\end{array}$ \\
\hline
\end{tabular}

a This includes the costs of: visits to a medical specialist, day care treatment, days hospitalized, visits to the emergency department and ambulance rides. ${ }^{b}$ This includes the costs of: visits to a medical assistant, occupational therapist, dietitian, speech therapist, physical therapist, psychologist, skin therapist, dentist, dental hygienist, pedicure, social worker, and alternative medicine. ' Informal care includes unpaid work such as domestic work, for example taking care of the children, grocery shopping, or volunteer work. 
Table 3. Incremental costs per quitter and per QALY gained for the incentive versus the control group, societal perspective.

\begin{tabular}{lcccccccc}
\hline & \multicolumn{4}{c}{ Total costs } & \multicolumn{3}{c}{ Total effects } \\
\hline Analysis $^{\mathbf{e}}$ & $\begin{array}{c}\text { Effect } \\
\text { measure }\end{array}$ & $\begin{array}{c}\text { Intervention } \\
\text { group }\end{array}$ & $\begin{array}{c}\text { Control } \\
\text { group }\end{array}$ & $\begin{array}{c}\boldsymbol{\Delta} \\
\text { costs }\end{array}$ & $\begin{array}{c}\text { Intervention } \\
\text { group }\end{array}$ & $\begin{array}{c}\text { Control } \\
\text { group }\end{array}$ & $\begin{array}{c}\boldsymbol{\Delta} \\
\text { effects }\end{array}$ & ICER $^{\mathbf{a}}$ \\
\hline $\begin{array}{l}\text { Cost- } \\
\text { effectiveness }\end{array}$ & $\begin{array}{c}\text { Quit } \\
\text { smoking }\end{array}$ & $€ 15,869$ & $€ 13,928$ & $€ 1,942$ & 0.44 & 0.27 & 0.17 & $€ 11,546$ \\
Cost-utility $^{\mathrm{g}}$ & QALYc & $€ 15,869$ & $€ 13,889$ & $€ 1,980$ & 1.055 & 1.070 & -0.02 & Dominated $^{\text {d }}$ \\
\hline
\end{tabular}

${ }^{a}$ Calculated based on the formula for ICER or ICUR, i.e. (Ci - CC) / (Ei - Ec)

${ }^{\mathrm{b}}$ Coded as $1=$ abstinent and $0=$ not abstinent

' Based on the Dutch tariff of the EQ-5D-5L

${ }^{\mathrm{d}}$ ICUR $=€ 128,280$

${ }^{\mathrm{e}}$ Only participants were included in the cost-effectiveness analyses who completed at least 1 cost questionnaire, therefore the data in this table differ from the RCT data.

fintervention group $n=289$, control group $n=273$. ${ }^{9}$ Intervention group $n=289$, control group $n=$ 274

Table 4. Results from cost-effectiveness analyses for the outcome quitting smoking for intervention and control group based on 5000 bootstrap replications: ICERS and distributions on the costeffectiveness plane.

\begin{tabular}{|c|c|c|c|c|c|}
\hline Sensitivity analyses ${ }^{\mathbf{a}}$ & ICER $^{\mathbf{b}}$ & North east & $\begin{array}{l}\text { South east } \\
\text { (dominant) }\end{array}$ & $\begin{array}{l}\text { South } \\
\text { west }\end{array}$ & $\begin{array}{l}\text { North west } \\
\text { (inferior) }\end{array}$ \\
\hline Society main analysis & $€ 11,546$ & 0.92 & 0.08 & 0.00 & 0.00 \\
\hline Society $95^{\text {th }}$ percentile & $€ 3,432$ & 0.78 & 0.22 & 0.00 & 0.00 \\
\hline Society complete case & $€ 17,610$ & 1.00 & 0.00 & 0.00 & 0.00 \\
\hline Society self-reported abstinence & $€ 12,826$ & 0.93 & 0.07 & 0.00 & 0.00 \\
\hline Employer main analysis & $€ 5,686$ & 0.83 & 0.16 & 0.00 & 0.00 \\
\hline Employer $95^{\text {th }}$ percentile & $€ 2,638$ & 0.73 & 0.26 & 0.00 & 0.00 \\
\hline Employer complete case & $€ 12,117$ & 0.99 & 0.01 & 0.00 & 0.00 \\
\hline Intervention costs only & $€ 1,124$ & 1.00 & 0.00 & 0.00 & 0.00 \\
\hline
\end{tabular}

a Society = analysis based on a societal perspective where all costs are included, Employer $=$ analysis based on an employer's perspective where only intervention costs and productivity costs are included. ${ }^{\mathrm{b}}$ Calculated based on the formula ICER $=(\mathrm{Ci}-\mathrm{Cc}) /(\mathrm{Ei}-\mathrm{EC})$ 


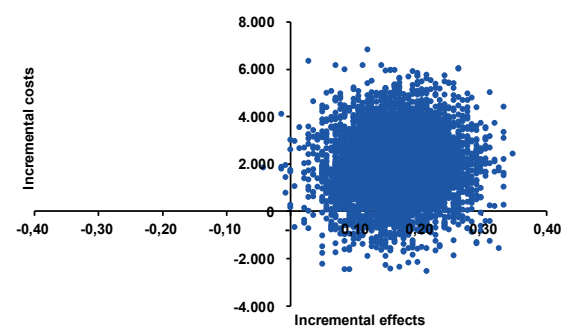

A. Main analysis from a societal perspective, cost per quitter.

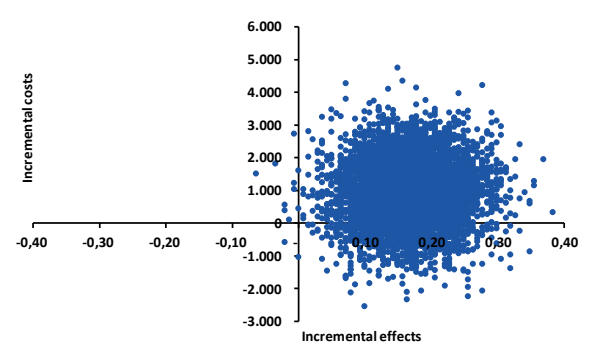

B. Main analysis from an employer's perspective, cost per quitter.

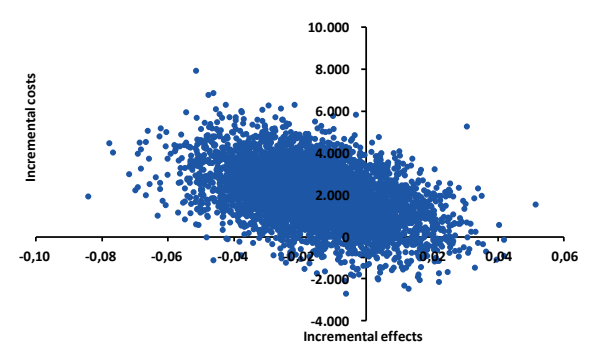

C. Main analysis from a societal perspective, cost per QALY.

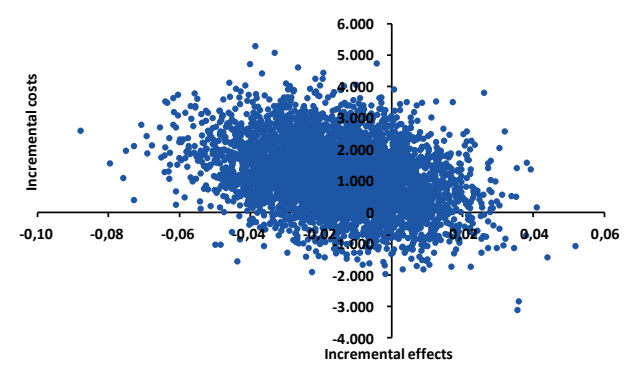

D. Main analysis from an employer's perspective, cost per QALY.
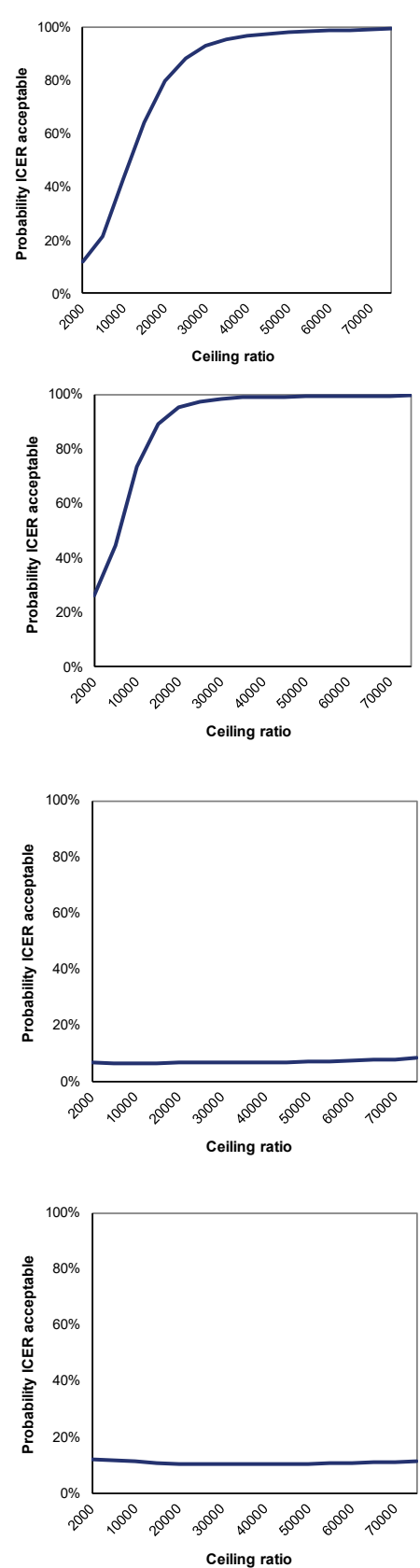

Figure 2. Cost-effectiveness planes (left) and corresponding cost-effectiveness acceptability curves (right) for the main analyses from a societal perspective and an employer's perspective for the outcomes quitting smoking and quality of life. 


\section{4-month cost-utility analyses}

Mean QALY scores were comparable between intervention and control group (1.05 vs 1.07, $p=0.205$ ), while the costs were higher in the intervention group for both the societal and the employer's perspective (Table 3). In the study, 172 of 563 participants (31\%) reported the maximum score of QALYs during the entire 14-month period (not shown in table). The cost-effectiveness planes of the main and sensitivity analyses show that the bootstrapped replications were distributed around the origin, and therefore no effect for QALYs could be detected (Figure $2 \mathrm{c}$ and $2 \mathrm{~d}$ ). The ICUR from the main analysis from a societal perspective was $€ 128,280$ and the ICUR from an employer's perspective was $€ 76,810$. With a ceiling ratio of $€ 20,000$ per QALY, this means that the intervention was dominated by the control condition for both the societal and the employer's perspective. The sensitivity analyses showed that the ICURs from a societal perspective varied between $€ 41,217$ and $€ 211,411$; the ICURS from an employer's perspective varied between $€ 12,249$ and $€ 145,921$ (not shown in table).

\section{Cost-effectiveness estimates from a lifetime perspective}

The estimation of costs from a lifetime perspective based on the ICER tables of Stapleton \& West $(2012)^{46}$, using an incremental intervention cost of $€ 189$, an incremental cessation percentage of 17.0 , and a discount rate of 3.5\%. Adjusting the results for the proportion of participants within the age categories $<35$ years old $(18.0 \%), 35-54$ years old $(61.8 \%)$, and $>54$ years old $(20.2 \%)$ showed an incremental cost per (quality adjusted) life year of $€ 1,249(95 \% \mathrm{Cl}=€ 850-€ 2,387)$. A more detailed overview of the calculations is presented in Supplementary file 2.

\section{DISCUSSION}

In the current study a cost-effectiveness and cost-utility analysis were performed alongside a randomized controlled trial involving financial incentives for smoking cessation. From a societal perspective, the results of the main cost-effectiveness analyses using a 14-month follow-up period showed that the intervention condition with incentives is cost-effective if society is willing to pay $€ 11,546$ for an additional quitter or if the employer is willing to pay $€ 5,686$ per quitter. The cost-effectiveness acceptability curves demonstrate that provided that the ceiling ratio is high enough (about $€ 20,000$ ), there is a high probability that the intervention will be cost effective.

The cost-utility analyses did not find an improvement in QALYs within the 14-month followup period. Given a willingness to pay of $€ 20,000$ per QALY, the intervention condition was dominated by the control condition for both the societal and the employer's perspective. The corresponding cost-effectiveness acceptability curves demonstrated that within the trial's follow-up period, the probability that the intervention was cost-effective was less than $10 \%$ at the $€ 20,000$ ceiling ratio. The estimates from a lifetime perspective, where ICER tables were used with input from the trial's cost- and effect data, showed an incremental cost per quality adjusted life year of $€ 1,249$. This amount is far below the willingness to pay threshold of $€ 20,000$ per QALY. ${ }^{45}$ 
The current study is the first full economic evaluation of a trial with incentives for smoking cessation at the workplace. Therefore, results cannot be compared to previous work. Because there is no accepted monetary cut-off point for smoking abstinence, it is difficult to conclude when a smoking cessation intervention is cost-effective. A study with pregnant women found incremental costs per additional quitter of $£ 1,127(€ 1,265){ }^{28}$ and three internet-based smoking cessation interventions conducted in the Netherlands showed ICERs between $€ 1,500$ and $€ 50,400$ (amounts uncorrected for inflation and time preferences). ${ }^{48-50}$ From the perspective of an employer, the willingness to pay for incentives for smoking cessation will for an important part depend on whether it eventually saves costs. In a study conducted in the United States, ${ }^{6}$ the annual excess cost of an employee who smokes was estimated to be $\$ 5,816$ ( $€ 5141$, uncorrected amount). Although costs in the Netherlands may differ and this amount needs to be interpreted as a general indication, it shows that an employer can benefit within a relatively short period of time by offering incentives and that it therefore may be a good investment.

The estimation of the incremental costs per life year, which we calculated using ICER tables which were developed to provide estimates of cost-effectiveness from a lifetime perspective, ${ }^{46}$ showed an ICER of $€ 1,249$. This result gives an indication that financial incentives may be a very cost-effective intervention from a long-term perspective.

Contrary to our hypothesis, we could not detect an improvement in quality of life within the 14-month follow-up period of the trial. An explanation for the lack of improvement of QALYs in the intervention group may be the withdrawal symptoms ${ }^{51}$ and anhedonia ${ }^{52}$ associated with smoking cessation, which would have been more prevalent in the intervention group with more successful quitters. Additionally, the time horizon of 14 months in the current trial may be too short to detect long term improvements in healthrelated quality of life, since previous research did find higher quality of life scores in smokers that had quit for one to three years and longer compared to current smokers. ${ }^{53-55}$ Of course, although quitting smoking can lead to short term health improvements such as a decrease in coughing and shortness of breath, ${ }^{56}$ the time frame of this study was too short to fully assess the most important health benefits of quitting smoking, namely the reduced risk on a life-threatening disease and mortality. ${ }^{2}$ In the pregnancy study of Boyd et al. (2015), the life-time benefits of quitting smoking expressed in QALYs were estimated using modelling techniques to estimate long-term cost-effectiveness and cost-utility and based on these results concluded that financial incentives were a cost-effective method to stimulate smoking cessation.

As may be expected from a general population of healthy employees, ${ }^{57}$ the QALY scores in both groups were very high, with an average baseline score of 0.90 and almost one third of the participants reporting the maximum utility score in the follow-up period. Therefore, there may have been a ceiling effect in a large proportion of the study population which prevented from finding improvements of QALY scores. ${ }^{58}$ As was suggested in previous research, the Euroqol-5D-5L may not have the discriminative power to detect changes in a healthy population. ${ }^{59}$ Changes in health-related quality of life have been detected within one year in studies using alternative measures such as positive affect, craving, and frequency of stressful events..$^{55,60}$ Therefore, a measuring instrument more specific 
to healthy employees should be developed that is sensitive enough to assess changes in health-related quality of life within the relatively short follow-up time of trial-based economic evaluation studies.

\section{Strengths and limitations}

To our best knowledge, this is the first study evaluating the cost-effectiveness and costutility of incentives for success in quitting in combination with a smoking cessation program at the workplace. A strength of this trial-based economic evaluation is that there is a direct link between the cost and effect data because these were measured within the same study population. Additionally, this study measured a broad array of health care costs, and included productivity losses and patient and family costs. The economic evaluation was performed from a societal perspective and from an employer's perspective, which increases relevance and applicability of the results. Another strength of this study is that the outcome smoking abstinence was not merely based on self-report, but was biochemically validated by an independent research assistant. The current study also has some limitations. The follow-up period of 14 months may have been too short to measure important health benefits related to smoking cessation, which should be addressed in future studies. Nevertheless, the cost-effectiveness estimates from a lifetime perspective based on ICER tables ${ }^{46}$ provide an indication that financial incentives may be a very cost-effective intervention on the long term. Still, a limitation of the ICER tables is that they only consider the costs of the intervention and not costs and benefits from a broader perspective such as an employer's or societal perspective. ${ }^{46}$ Another limitation of the current study was that the incentives were still in place at the final follow up moment. However, previous studies and a meta-analysis of financial incentives for smoking cessation have provided evidence for a sustained effect of financial incentives after the incentives were removed. ${ }^{15,16,61}$ Because a proportion of the participants did not complete all questionnaires, some cost data had to be imputed. However, the complete case analysis showed comparable results, which strengthens confidence in the data. Furthermore, cost data were based on self-report which may be prone to bias, and the retrospective nature of the questionnaires may have led to recall bias, which may have caused an over- or underestimation of costs. ${ }^{62} \mathrm{~A}$ systematic review comparing self-reports and administrative data to measure health care resource use for cost estimation found that, although patients may report lower resource use, self-reported data may provide adequate estimates when administrative data are not available. ${ }^{63}$ Regarding the reliability and validity of self-reports to measure absenteeism from work, a meta-analysis found, compared to employer records, a tendency to under-report absenteeism, which was reduced if the self-report was focused specifically on absence due to sickness, as was done in the current study. ${ }^{64}$ Moreover, in the current study, a potential bias in the reporting of resource use is expected to be comparable for both the intervention and control group. A final limitation is that the current study was designed to include a limited number of employees within each company. To maximize the impact of financial incentives, it should be further investigated how the reach of the intervention among employees can be increased. 


\section{Conclusion}

Financial incentives were cost-effective in increasing the number of successful quitters from a societal and from an employer's perspective within a 14-month time frame, depending on how much society or the employer is willing to pay for an additional quitter. The results of the cost-utility analysis within the 14-month time frame were inconclusive, likely due to the relatively short observation period. The results from a lifetime perspective showed that financial incentives were far below the willingness to pay threshold of $€ 20.000$ per QALY.

The current study demonstrates that providing financial incentives for smoking cessation may be cost-effective within a short time period, and may help policy makers and employers decide on implementing incentives to stimulate smoking cessation. 


\section{REFERENCES}

1. WHO. WHO report on the global tobacco epidemic, 2008: the MPOWER package. 2008.

2. US Department of Health and Human Services. The health consequences of smoking-50 years of progress. In. a report of the Surgeon General. Vol 17. Atlanta, GA: US Department of Health and Human Services, Centers for Disease Control and Prevention, National Center for Chronic Disease Prevention and Health Promotion, Office on Smoking and Health; 2014.

3. Jha P, Ramasundarahettige C, Landsman V, et al. 21st-Century Hazards of Smoking and Benefits of Cessation in the United States. N Engl J Med. 2013;368(4):341-350.

4. Halpern MT, Shikiar R, Rentz AM, Khan ZM. Impact of smoking status on workplace absenteeism and productivity. Tob Control. 2001;10(3):233-238.

5. Tsai S, Wen C, Hu S, Cheng T, Huang S. Workplace smoking related absenteeism and productivity costs in Taiwan. Tob Control. 2005;14(suppl 1):i33-i37.

6. Berman M, Crane R, Seiber E, Munur M. Estimating the cost of a smoking employee. Tob Control. 2014;23.

7. Troelstra SA, Coenen P, Boot CR, Harting J, Kunst AE, van der Beek AJ. Smoking and sickness absence: a systematic review and meta-analysis. Scand J Work Environ Health. 2019.

8. Hartmann-Boyce J, Stead LF, Cahill K, Lancaster T. Efficacy of interventions to combat tobacco addiction: Cochrane update of 2012 reviews. Addiction. 2013;108.

9. Cahill K, Stevens S, Perera R, Lancaster T. Pharmacological interventions for smoking cessation: an overview and network meta-analysis. Cochrane Database Syst Rev. 2013(5):CD009329.

10. Stead LF, Koilpillai P, Fanshawe TR, Lancaster T. Combined pharmacotherapy and behavioural interventions for smoking cessation. Cochrane Database of Systematic Reviews. 2016(3).

11. Cahill K, Lancaster T. Workplace interventions for smoking cessation. The Cochrane Library. 2014.

12. Hiscock R, Dobbie F, Bauld L. Smoking Cessation and Socioeconomic Status: An Update of Existing Evidence from a National Evaluation of English Stop Smoking Services. Biomed Res Int. 2015;2015:274056.

13. Huisman M, Kunst AE, Mackenbach JP. Inequalities in the prevalence of smoking in the European Union: comparing education and income. Prev Med. 2005;40.

14. Hill S, Amos A, Clifford D, Platt S. Impact of tobacco control interventions on socioeconomic inequalities in smoking: review of the evidence. Tob Control. 2014;23(e2):e89-e97.

15. Halpern SD, Harhay MO, Saulsgiver K, Brophy C, Troxel AB, Volpp KG. A Pragmatic Trial of E-Cigarettes, Incentives, and Drugs for Smoking Cessation. N Engl J Med. 2018.

16. Volpp KG, Troxel AB, Pauly MV, et al. A Randomized, Controlled Trial of Financial Incentives for Smoking Cessation. N Engl J Med. 2009;360(7):699-709.

17. van den Brand FA, Nagelhout GE, Winkens B, Chavannes NH, van Schayck OCP. Effect of a workplace-based group training programme combined with financial incentives on smoking cessation: a cluster-randomised controlled trial. The Lancet Public health. 2018;3(11):e536-e544.

18. Smith AL, Carter SM, Chapman S, Dunlop SM, Freeman B. Why do smokers try to quit without medication or counselling? A qualitative study with ex-smokers. BMJ Open. 2015;5(4):e007301.

19. Carroll C, Rick J, Leaviss J, Fishwick D, Booth A. A qualitative evidence synthesis of employees' views of workplace smoking reduction or cessation interventions. BMC Public Health. 2013;13:1095.

20. Van den Brand FA, Dohmen LME, Van Schayck OCP, Nagelhout GE. 'Secretly, it's a competition': a qualitative study investigating what helped employees quit smoking during a workplace smoking cessation group training programme with incentives. BMJ Open. 2018;8(11):e023917. 
21. Weng SF, Ali S, Leonardi-Bee J. Smoking and absence from work: systematic review and metaanalysis of occupational studies. Addiction. 2013;108(2):307-319.

22. Eriksen MP, Gottlieb NH. A review of the health impact of smoking control at the workplace. Am $J$ Health Promot. 1998;13(2):83-104.

23. Osinubi OY, Slade J. Tobacco in the workplace. Occup Med. 2002;17(1):137-158, vi.

24. Berman M, Crane R, Seiber E, Munur M. Estimating the cost of a smoking employee. Tobacco Control. 2014;23(5):428-433.

25. Van den Brand FA, Nagelhout GE, Winkens B, et al. The effect of financial incentives on top of behavioral support on quit rates in tobacco smoking employees: study protocol of a clusterrandomized trial. BMC Public Health. 2016;16(1):1056.

26. Promberger M, Dolan P, Marteau TM. "Pay them if it works": Discrete choice experiments on the acceptability of financial incentives to change health related behaviour. Soc Sci Med. 2012;75(12):2509-2514.

27. Giles EL, Robalino S, Sniehotta FF, Adams J, McColl E. Acceptability of financial incentives for encouraging uptake of healthy behaviours: A critical review using systematic methods. Prev Med. 2015;73:145-158.

28. Boyd KA, Briggs AH, Bauld L, Sinclair L, Tappin D. Are financial incentives cost-effective to support smoking cessation during pregnancy? Addiction. 2016;111(2):360-370.

29. Sung H-Y, Penko J, Cummins SE, et al. Economic Impact of Financial Incentives and Mailing Nicotine Patches to Help Medicaid Smokers Quit Smoking: A Cost-Benefit Analysis. Am J Prev Med. 2018;55(6, Supplement 2):S148-S158.

30. Max W. The Financial Impact of Smoking on Health-Related Costs: A Review of the Literature. Am J Health Promot. 2001;15(5):321-331.

31. Husereau $D$, Drummond $M$, Petrou $S$, et al. Consolidated health economic evaluation reporting standards (CHEERS) statement. 2013;11(1):6.

32. Hakkaart-van Roijen L, Van der Linden N, Bouwmans C, Kanters T, Swan Tan SK. Methodologie van kostenonderzoek en referentieprijzen voor economische evaluaties in de gezondheidszorg. . Institute for Medical Technology Assessment Erasmus Universiteit Rotterdam. 2015.

33. Drummond MF, Sculpher MJ, Claxton K, Stoddart GL, Torrance GW. Methods for the economic evaluation of health care programmes. Oxford university press; 2015.

34. Thorn J, Coast J, Cohen D, et al. Resource-Use Measurement Based on Patient Recall: Issues and Challenges for Economic Evaluation. Applied Health Economics and Health Policy. 2013;11 (3):155161.

35. Bouwmans $C$, Krol M, Severens $H$, Koopmanschap M, Brouwer W, Roijen LH-v. The iMTA Productivity Cost Questionnaire: A Standardized Instrument for Measuring and Valuing HealthRelated Productivity Losses. Value Health. 2015;18(6):753-758.

36. Bouwmans C, Hakkaart-van Roijen L, Koopmanschap M, Krol M, Severens H, Brouwer W. Medical Cost Questionnaire (iMCQ). In: Rotterdam: iMTA, Erasmus Universiteit Rotterdam; 2013.

37. Dirum. Database of instruments for resource use management. www.dirum.org Published 2016. Accessed on 15 July 2016.

38. Tan SS, Bouwmans CAM, Rutten FFH, Hakkaart-van RL. Update of the Dutch manual for costing in economic evaluations. Int J Technol Assess Health Care. 2012;28.

39. West RPLJ. Outcome criteria in smoking cessation trials: proposal for a common standard. Addiction. 2005;100.

40. Group TE. EuroQol-a new facility for the measurement of health-related quality of life. Health Policy. 1990;16(3):199-208. 
41. Versteegh MM, Vermeulen KM, Evers SM, de Wit GA, Prenger R, Stolk EA. Dutch Tariff for the FiveLevel Version of EQ-5D. Value in health. 2016.

42. Manca A, Hawkins N, Sculpher MJ. Estimating mean QALYs in trial-based cost-effectiveness analysis: the importance of controlling for baseline utility. Health Econ. 2005;14(5):487-496.

43. Faria R, Gomes M, Epstein D, White IR. A Guide to Handling Missing Data in CostEffectiveness Analysis Conducted Within Randomised Controlled Trials. Pharmacoeconomics. 2014;32(12):1157-1170.

44. Briggs $\mathrm{AH}$, Wonderling $\mathrm{DE}$, Mooney CZ. Pulling cost-effectiveness analysis up by its bootstraps: A non-parametric approach to confidence interval estimation. Health Econ. 1997;6(4):327-340.

45. Zwaap J, Knies S, Van der Meijden C, Staal P, Van der Heiden L. Kosteneffectiviteit in de praktijk. Zorginstituut Nederland, 2015. 2015.

46. Stapleton JA, West R. A direct method and ICER tables for the estimation of the cost-effectiveness of smoking cessation interventions in general populations: application to a new cytisine trial and other examples. Nicotine \& tobacco research : official journal of the Society for Research on Nicotine and Tobacco. 2012;14(4):463-471.

47. West R, Hajek P, Stead L, Stapleton J. Outcome criteria in smoking cessation trials: proposal for a common standard. Addiction. 2005;100(3):299-303.

48. Schulz DN, Smit ES, Stanczyk NE, Kremers SP, de Vries H, Evers SM. Economic evaluation of a web-based tailored lifestyle intervention for adults: findings regarding cost-effectiveness and cost-utility from a randomized controlled trial. J Med Internet Res. 2014;16(3):e91.

49. Smit ES, Evers SMAA, de Vries H, Hoving C. Cost-Effectiveness and Cost-Utility of Internet-Based Computer Tailoring for Smoking Cessation. J Med Internet Res. 2013;15(3):e57.

50. Stanczyk NE, Smit ES, Schulz DN, et al. An economic evaluation of a video- and text-based computer-tailored intervention for smoking cessation: a cost-effectiveness and cost-utility analysis of a randomized controlled trial. PLoS One. 2014;9(10):e110117.

51. Shiffman S, Patten C, Gwaltney C, et al. Natural history of nicotine withdrawal. Addiction. 2006;101(12):1822-1832.

52. Cook JW, Piper ME, Leventhal AM, Schlam TR, Fiore MC, Baker TB. Anhedonia as a component of the tobacco withdrawal syndrome. J Abnorm Psychol. 2015;124(1):215-225.

53. Mulder I, Tijhuis M, Smit HA, Kromhout D. Smoking Cessation and Quality of Life: The Effect of Amount of Smoking and Time Since Quitting. Prev Med. 2001;33(6):653-660.

54. Heikkinen $\mathrm{H}$, Jallinoja P, Saarni SI, Patja K. The impact of smoking on health-related and overall quality of life: a general population survey in Finland. Nicotine \& tobacco research : official journal of the Society for Research on Nicotine and Tobacco. 2008;10(7):1199-1207.

55. Piper ME, Kenford S, Fiore MC, Baker TB. Smoking cessation and quality of life: changes in life satisfaction over 3 years following a quit attempt. Ann Behav Med. 2012;43(2):262-270.

56. US Department of Health and Human Services. The health benefits of smoking cessation. Center for Chronic Disease Prevention and Health Promotion, Office on Smoking and Health. 1990.

57. Versteegh MM, Vermeulen KM, Evers SM, Wit GA, Prenger R, Stolk EA. Dutch Tariff for the FiveLevel Version of EQ-5D. Value Health. 2016;19.

58. Ferreira LN, Ferreira PL, Ribeiro FP, Pereira LN. Comparing the performance of the EQ-5D-3L and the EQ-5D-5L in young Portuguese adults. Health and Quality of Life Outcomes. 2016;14(1):89.

59. Konnopka A, Koenig H-H. The "no problems"-problem: an empirical analysis of ceiling effects on the EQ-5D 5L. Qual Life Res. 2017;26(8):2079-2084.

60. Schlam TR, Piper ME, Cook JW, Fiore MC, Baker TB. Life 1 Year After a Quit Attempt: Real-Time Reports of Quitters and Continuing Smokers. Ann Behav Med. 2012;44(3):309-319. 
61. Notley C, Gentry S, Livingstone-Banks J, Bauld L, Perera R, Hartmann-Boyce J. Incentives for smoking cessation. Cochrane Database of Systematic Reviews. 2019(7).

62. Brusco NK, Watts JJ. Empirical evidence of recall bias for primary health care visits. BMC Health Serv Res. 2015;15:381.

63. Noben CY, de Rijk A, Nijhuis F, Kottner J, Evers S. The exchangeability of self-reports and administrative health care resource use measurements: assessement of the methodological reporting quality. J Clin Epidemiol. 2016;74:93-106.e102.

64. Johns G, Miraglia M. The reliability, validity, and accuracy of self-reported absenteeism from work: a meta-analysis. J Occup Health Psychol. 2015;20(1):1-14. 


\section{Supplementary file 1: Valuation of costs}

Intervention costs consisted in part of the smoking cessation program, which was $€ 4206$, independent of the training attendance and the number of participants per group (8 to 16 participants). Costs for the smoking cessation validation device $\left(\mathrm{PiCO}^{\mathrm{TM}}\right.$ Smokerlyzer, Bedfont Scientific Ltd., Kent, England) were $€ 351$, but were left out of the analysis due to one-off purchase costs. The cost of the financial incentives was based on actual individual success in quitting, and was a maximum of $€ 350$ euros for each participant who remained abstinent throughout the entire follow-up period. To account for the time that employees spent participating in the training, the average hourly rate of $€ 35.33^{1}$ was multiplied with the maximum attendance of seven training sessions of 90 minutes plus an additional four times ten minutes for performing the follow-up CO measurements.

To determine patient and family costs, travel costs for visits to health care services were calculated by taking the average distance to the specific health care services in the Netherlands ${ }^{1}$ and multiplying this with fuel costs of $€ 0.19 / \mathrm{km}$. For parking costs, $€ 3.07$ was counted per visit.' Since the smoking cessation program and follow-up measurements were conducted at the workplace, no travelling costs were calculated for the intervention. To account for the time spent on informal care, replacement costs were calculated by the standard hourly rate of cleaning work, as recommended by Dutch guidelines, ${ }^{1}$ which was 14.32 euros per hour.

Health care costs were estimated using a bottom-up (or micro-costing) approach, where the total cost of each individual unit of used health care was multiplied by an appropriate unit cost and summed to provide an overall total cost. If costs of health care treatments were not available from the Dutch guidelines, ${ }^{1}$ they were calculated based on the average price listed by three different hospitals. Medication costs were based on the lowest priced version listed on the Dutch healthcare institute website (www.medicijnkosten.nl) for the cost of pharmaceuticals. For medications that were sold over the counter, the sales price of a standard package was counted, while for prescription medications, costs were calculated based on the dose reported by the participants. If information on treatments or medication was incomplete, the smallest possible dose was counted. For prescription medications, an extra rate for the dispensing of the medication was added to the costs, which was 7 euros for chronic medication and 14 euros for medication that was prescribed for less than 30 days.'

As a part of estimating productivity costs, cost of absenteeism from paid work due to sick leave was calculated using the friction costs method, ${ }^{1,2}$ which assumes that employees will be replaced after a period of absence, which was calculated to be 85 calendar days ( 12 weeks) on average in the Netherlands. Therefore, for longer absence periods, a maximum of 85 days was used.' ${ }^{1}$ Costs of productivity losses due to presenteeism were calculated by multiplying the percentage of the normal work that an employee could do with the number of work days the employee reported to have been less able to do the normal amount of work. Productivity costs related to work breaks were calculated based on the participants' self-report of number and duration of breaks. To calculate the productivity costs per hour per paid employee, the standard tariff of $€ 35.33$ was counted. ${ }^{1}$ 
1. Hakkaart-van Roijen L, Van der Linden N, Bouwmans C, Kanters T, Swan Tan SK. Methodologie van kostenonderzoek en referentieprijzen voor economische evaluaties in de gezondheidszorg. Institute for Medical Technology Assessment Erasmus Universiteit Rotterdam. 2015.

2. Van den Hout W. The value of productivity: human-capital versus friction-cost method. Ann Rheum Dis. 2010;69(Suppl 1):i89-i91.

\section{Supplementary file 2: calculation of ICER from a lifetime perspective based on ICER tables}

\section{Calculation input data}

\begin{tabular}{lc}
\hline Incremental intervention effect & $\mathbf{0 . 1 6 8} \mathbf{( 9 5 \% ~ C l = 0 . 0 8 9 - 0 . 2 4 4 )}$ \\
\hline Intervention costs & $€ 189$ \\
Proportion of participants within age categories: & \\
<35 years old & $18.0 \%$ \\
35-54 years old: & $61.8 \%$ \\
55 years and older: & $20.2 \%$ \\
Multiplication factors for age categories: & \\
$<35$ years old & 1.46 \\
35-54 years old: & 1.00 \\
55 years and older & 1.36 \\
Discount rate per annum & $3.5 \%$ \\
Follow up period & 12 months \\
ICER from Table 3B Stapleton \& West (2012) for a intervention & \\
costs of $€ 200$ & \\
Effect size 17\% & $€ 1144$ \\
$95 \%$ Cl 9\% - 25\% & $€ 778-€ 2161$ \\
\hline
\end{tabular}

Calculation of ICER based on Table 3B of Stapleton \& West (2012) (1)

Calculating ICER for intervention costs of $€ 189$ :

$\operatorname{ICER}(17 \%$ incremental intervention effect $)=189 *(1144 / 200)=1,081.08$

Adjusting ICER for age categories:

$0.180 *(1,081.08 * 1.46)=284.11$

$0.618 *(1,081.08 * 1)=668.11$

$0.202 *(1,081.08 * 1.36)=296.99$

ICER $=284.11+668.11+296.99=\underline{\mathbf{1 , 2 4 9 . 2 1}}$

Calculating $95 \% \mathrm{Cl}$ of ICER

ICER LL (25\% incremental intervention effect): $189 *(778 / 200)=735.21$

ICER UL (9\% incremental intervention effect): $189 *(2161 / 200)=2042.145$ 


\section{Adjusting ICER of $\mathbf{9 5 \%} \mathrm{Cl}$ for age categories:}

$0.180 *(735.21 * 1.46)=193.21$

$0.618 *(735.21 * 1)=454.36$

$0.202 *(735.21 * 1.36)=201.98$

ICER 95\% CI LL $=\underline{\mathbf{8 4 9 . 5 5}}$

$0.180 *(2042.15 * 1.46)=563.65$

$0.618 *(2042.15 * 1)=1262.05$

$0.202 *(2042.15 * 1.36)=561.02$

ICER 95\% CI UL = $\underline{\mathbf{2}, \mathbf{3 8 6 . 7 2}}$

ICER = 1,249 $(95 \% \mathrm{Cl}=850-2,387)$

1. Stapleton JA, West R. A direct method and ICER tables for the estimation of the cost-effectiveness of smoking cessation interventions in general populations: application to a new cytisine trial and other examples. Nicotine \& tobacco research : official journal of the Society for Research on Nicotine and Tobacco. 2012;14(4):463-71. 


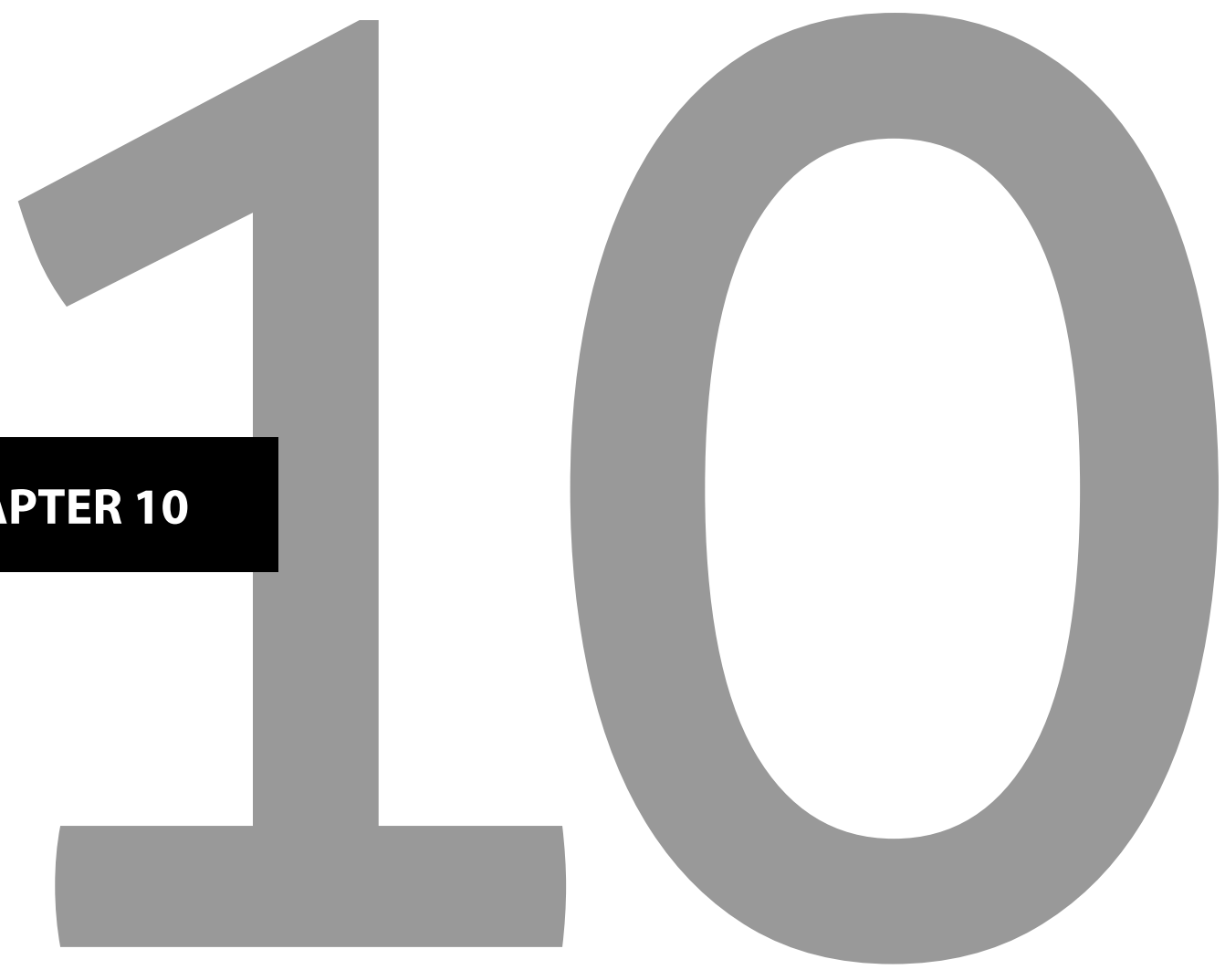


\section{Implementation of financial incentives for successful smoking cessation in real-life company settings: a qualitative needs assessment among employers}

Van den Brand FA, Magnée T, de Haan-Bouma L, et al. Implementation of Financial Incentives for Successful Smoking Cessation in Real-Life Company Settings: A Qualitative Needs Assessment among Employers 


\section{ABSTRACT}

Randomized studies have shown that financial incentives can significantly increase the effect of smoking cessation treatment in company settings. Evidence of effectiveness alone is, however, not enough to ensure that companies will offer this intervention. Knowledge about the barriers and facilitators for implementation in the workplace is needed, in order to develop an implementation strategy. We performed a qualitative needs assessment among 18 employers working in companies with relatively many employees with a low educational level, and our study revealed priority actions that aim to improve the implementation process in these types of workplaces. First, employers need training and support in how to reach their employees and convince them to take part in the group training. Second, employers need to be convinced that their nonsmoking employees will not consider the incentives unfair, or they should be enabled to offer alternative incentives that are considered less unfair. Third, the cost-effectiveness of smoking cessation group trainings including financial incentives should be explained to employers. Finally, smoking cessation should become a standard part of workplace-based health policies. 


\section{INTRODUCTION}

Workplaces can be a good setting for health promotion, ${ }^{1}$ such as for helping people to quit smoking. For employers, it is likely that it pays off financially to stimulate healthy behavior, such as non-smoking, among their employees. ${ }^{2}$ Randomized studies have shown that financial incentives can significantly increase the effect of smoking cessation treatment in company settings. ${ }^{3,4}$ Evidence of effectiveness alone is, however, not enough to ensure that companies will offer financial incentives to their smoking employees. Offering financial incentives is already quite common in companies in the United States, ${ }^{5,6}$ but in Europe this is much less common, possibly because of differences in culture and in health insurance system. ${ }^{7}$ Knowledge is needed about barriers and facilitators for implementation of financial incentives for successful smoking cessation in the workplace in order to inform implementation strategies. ${ }^{8,9}$

The most important difference between using financial incentives in a randomized study setting and implementing them in real-life is who pays the financial incentives: the employer needs to pay instead of the funder of the study. Although employees who quit smoking are potentially a cost-saver for companies, ${ }^{10}$ the immediate costs of the financial incentives can still be felt as a barrier to implementation. Another often mentioned barrier to real-life implementation of financial incentives is the perceived unfairness of the intervention towards employees who do not smoke and cannot earn the incentive. ${ }^{11,12}$ It is important to find out which solutions employers see to tackle these barriers and which facilitating factors may be present or could be created in companies.

Smoking cessation treatment with financial incentives can potentially contribute to the pressing issue of increasing socioeconomic inequalities in health. Studies have shown that financial incentives can work as well among people with a low socioeconomic status (SES) as among people with a higher socioeconomic status, ${ }^{3,4}$ while many other tobacco control interventions work better among people with a higher SES. ${ }^{13,14}$ Smoking cessation treatment with financial incentives at the workplace can help to decrease socioeconomic inequalities if it is being implemented more in companies with relatively many employees with a lower SES and if employees with a lower SES sign up to participate in the intervention more than employees with a higher SES. Knowledge of barriers and facilitators to the implementation of financial incentives in the specific context of lower SES employees and blue-collar workplaces is needed to target implementation strategies. ${ }^{13}$

The qualitative needs assessment that is described in this paper, is performed among employers in the Netherlands. In the Netherlands, $26 \%$ of lower educated people smoke compared to $17 \%$ of higher educated people. ${ }^{15}$ Additionally, nine out of ten lower educated smokers smoke daily, while only half of higher educated smokers smoke daily. Smoking is not allowed inside workplaces in the Netherlands, but designated indoor smoking rooms are allowed. ${ }^{16}$ Since a few years, there is a societal call for a 'smokefree generation' and some employers have started to make their company smokefree. This generally means closing the designated smoking rooms and banning smoking near the entrance of the building. Oftentimes, employees who smoke are also offered smoking cessation treatment when companies become smokefree. Additionally, in the national Prevention Agreement 
that was published in 2018, the policy intention is expressed to ban smoking rooms in all workplaces in 2023 and to stimulate smokefree companies in the meantime. ${ }^{17}$ These societal and political developments in the Netherlands are relevant because they could accelerate the implementation process of financial incentives for smoking cessation.

To gain insight into the conditions under which smoking cessation treatment with financial incentives is acceptable and how this could be implemented in real-life company settings, we performed a qualitative needs assessment among workplaces with relatively many employees with a low educational level. Our research question was: What are barriers and facilitators for the reach, adoption, implementation, and maintenance of smoking cessation group trainings and for providing financial incentives among employers?

\section{METHODS}

\section{Design}

We performed qualitative interviews $(n=18)$ among employers of organizations in the Netherlands where relatively many employees with a low educational level work. Interviews were performed face-to-face between January and June 2019. A qualitative design was chosen because it allowed us to ask open-ended questions and explore and understand barriers and facilitators.

\section{Sample}

Employers worked at a company with at least 100 employees where relatively many people with a low educational level are employed. Employers were those people in the organization who advised or decided about the possible implementation of smoking cessation group trainings and about whether to use financial incentives. This was not always a director or manager of an organization, but could also be someone working at human resource management or workplace health and safety (they are all called 'employers' in this article).

Participants were purposively selected to ensure variation in type of organizations and type of work. See Table 1 for the sector types participants worked in. We recruited part of the respondents from a previous study in which smoking cessation group trainings with financial incentives were performed. ${ }^{3}$ In this previous study, the group trainings were paid for by the companies, and the financial incentives by the funder of the study. Another part of the respondents for the current study were recruited by emailing and calling companies ourselves. Finally, there were some companies who contacted us because they wanted to know more about smoking cessation group trainings with financial incentives (because of media attention about our previous study). Participants received a flyer about the study with information about the interviews and signed an informed consent form before the interview started. As a compensation for the interview, participants received a fee of $€ 20$ euros that was deposited into their bank account. 
Table 1. Characteristics of respondents $(n=18)$.

\begin{tabular}{lc}
\hline & $\begin{array}{c}\text { Employers } \\
\mathbf{n}(\%)\end{array}$ \\
\hline Gender & $11(59)$ \\
Male & $7(41)$ \\
Female & \\
Age & $1(6)$ \\
$18-29$ years & $1(6)$ \\
$30-39$ years & $4(22)$ \\
$40-49$ years & $9(50)$ \\
$50-59$ years & $1(6)$ \\
60 years and older & $2(11)$ \\
Unknown & \\
Job title & $2(11)$ \\
Director / higher management & $8(44)$ \\
Human resources manager & $6(33)$ \\
Health and safety advisor/ consultant & $1(6)$ \\
Occupational physician & $1(6)$ \\
Vitality coach & \\
Size of organization & $1(6)$ \\
100-250 employees & $8(44)$ \\
$250-1,000$ employees & $5(28)$ \\
1,000-2,500 employees & $4(22)$ \\
S2,500 employees & \\
Goctor & $3(17)$ \\
Semi-government & $2(11)$ \\
Educational & $3(17)$ \\
Industrial (chemical/metal/energy/horticulture) & $7(39)$ \\
Health care & $2(11)$ \\
Financial & $1(6)$ \\
\hline
\end{tabular}

\section{Data collection}

Interviews were conducted at the workplace by one of the authors (CB), who is trained and experienced in qualitative interviewing. Interviews lasted on average 53 minutes (ranging between 15 minutes and 69 minutes).

The interview guide (see Supplementary file) was semi-structured and based on the RE-AIM Framework. ${ }^{8}$ RE-AIM is an acronym for: reach, effectiveness, adoption, implementation, and maintenance. Because we have investigated the effectiveness of smoking cessation treatment in combination with incentives in previous work, ${ }^{3}$ it was not included in the current study. Employers received questions like 'how can you reach your employees with an offer of a group smoking cessation training?' (reach), 'what are barriers for the implementation of financial incentives?' (adoption), 'how would you implement incentives when they are no longer paid by a research grant?' (implementation), and 'would you want to keep offering group trainings and incentives to your employees?' (maintenance). 
Because some respondents were recruited from the previous study about smoking cessation group trainings with financial incentives they received questions about their previous experiences, and other respondents received questions about a hypothetical situation in which group trainings and incentives would be implemented. Additionally, because the respondents had not (yet) implemented smoking cessation trainings with incentives, the current study assessed for 'adoption' the (anticipated) factors associated with organizational support and the willingness to implement the intervention. For 'implementation', instead of whether the intervention was delivered properly, we assessed how employers would want to implement the intervention and what would be potential barriers and facilitators for the implementation. Respondents were also asked some questions about their sociodemographic characteristics, organization and job title (see Table 1).

\section{Analysis}

Interviews were audio recorded and transcribed verbatim. The interviewer (CB) wrote memos and an overall report of observations before all interviews were finalized and the formal coding process started. Analyses were performed using NVivo version 12.

Thematical coding according to the Framework Method was applied. ${ }^{18}$ The Framework Method has five stages: (1) familiarization, (2) identifying the thematic framework, (3) indexing, (4) charting, and (5) mapping and interpretation. The familiarization stage was done by $C B$ through reading through memos and writing an overall report. This stage was done by GN, TM, LB, and FvdB through reading the overall report of observations and some of the transcripts before proceeding to stage 2. The thematic framework was made by $C B$ and TM and was slightly adjusted after discussions with GN and LB. Two authors (TM and LB) performed the indexing stage (applying the thematic framework on all transcripts) independently for the first two interviews. The codes that were used emerged both deductively from the interview guide and inductively from the transcripts. After agreement was reached about a final thematic framework, LB and TM separately completed the coding of the remaining interviews. Unclear passages were discussed until consensus was reached between coders. The charting stage consisted of making matrices, in which the responses on the most mentioned codes of the respondents were summarized using the RE-AIM elements. In the mapping and interpretation stage, the matrices were examined closely to make comparisons between codes within respondents and comparisons between respondents within codes. Full transcripts were regularly consulted to check the original wordings and contexts of remarks. The final two stages of the analyses were performed by GN and FvdB. 


\section{RESULTS}

Current availability of group trainings and incentives at the interviewed companies Among all respondents, seven employers had been in the previous RCT's intervention group with financial incentives, and two employers had been in the control group without incentives but with the group trainings. Of these nine employers, four had the intention to provide smoking cessation support in the future, but only one employer was offering group trainings at the time of the interview. Only a single employer from the control group was planning to offer financial incentives for quit success. There were no apparent distinctions in responses based on the demographic characteristics of the respondents. In the following paragraphs, the barriers and facilitators for the reach, adoption, implementation and maintenance of group trainings and incentives are described. An overview of the barriers and facilitators is presented in Table 2. 


\begin{tabular}{|c|c|c|}
\hline Barriers & Priority actions & Facilitators \\
\hline \multicolumn{3}{|c|}{ Reach } \\
\hline 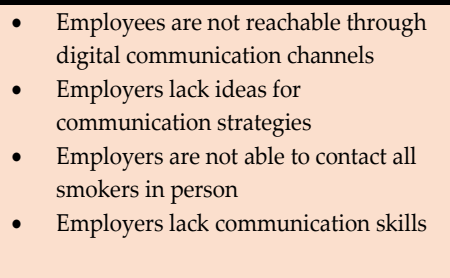 & $\begin{array}{l}\text { Training and helping } \\
\text { employers in how to } \\
\text { reach their employees } \\
\text { and convince them to } \\
\text { take part in the group } \\
\text { training }\end{array}$ & $\begin{array}{l}\text { Combining multiple communication } \\
\text { strategies } \\
\text { Individual approach through team } \\
\text { leaders } \\
\text { Reaching employees through word of } \\
\text { mouth } \\
\text { Organizing an information meeting for } \\
\text { employees }\end{array}$ \\
\hline \multicolumn{3}{|c|}{ Adoption } \\
\hline $\begin{array}{l}\text { Financial benefits of smoking } \\
\text { cessation are unclear } \\
\text { - } \quad \text { Disappointment in cessation outcomes } \\
\text { - } \quad \text { Incentives are considered unfair } \\
\text { - Incentives are not consistent with } \\
\text { peoples' values }\end{array}$ & $\begin{array}{l}\text { that their emplo } \\
\text { will not conside } \\
\text { incentives unfair } \\
\text { coming up wi } \\
\text { alternative incen }\end{array}$ & $\begin{array}{l}\text { - Responsibility for the health of } \\
\text { employees } \\
\text { Becoming a smoke-free company is an } \\
\text { opportunity } \\
\text { - Annoyance about smoking breaks } \\
\text { Believing that financial incentives are } \\
\text { effective } \\
\text { Recognizing the cost-benefit of } \\
\text { financial incentives } \\
\text { Making financial incentives fairer }\end{array}$ \\
\hline \multicolumn{3}{|c|}{ Implementation } \\
\hline $\begin{array}{l}\text { - The costs of trainings and incentives } \\
\text { - Limited time and resources }\end{array}$ & $\begin{array}{l}\text { Explaining the cost- } \\
\text { effectiveness of } \\
\text { smoking cessation } \\
\text { group trainings } \\
\text { including financial } \\
\text { incentives }\end{array}$ & $\begin{array}{l}\text { - Having an insurance company that } \\
\text { reimburses the group trainings and } \\
\text { incentives } \\
\text { - Participation of the partner of the } \\
\text { employee } \\
\text { Alternatives for group trainings } \\
\text { - Trainings within working hours }\end{array}$ \\
\hline \multicolumn{3}{|c|}{ Maintenance } \\
\hline $\begin{array}{l}\text { - } \quad \text { Smoking is not included in the } \\
\text { existing health promotion program } \\
\text { - Little enthusiasm for smoking } \\
\text { cessation among employees }\end{array}$ & $\begin{array}{l}\text { Ensuring that smoking } \\
\text { cessation becomes a } \\
\text { part of existing health } \\
\text { promotion programs }\end{array}$ & $\begin{array}{l}\text { Making smoking cessation part of } \\
\text { larger health promotion program }\end{array}$ \\
\hline
\end{tabular}

Table 2. Overview of barriers and facilitators that were mentioned by employers and the actions that should be prioritized in order to improve the implementation process in workplaces with relatively many employees with a low educational level. 


\subsection{Reach: how can you reach your employees with an offer of a group smoking cessation training?}

\subsubsection{Barriers for the reach of the program}

Employees are not reachable through digital communication channels

Many employers mentioned that they have office workers who could be reached relatively easy, but that they have also employees who do not work behind a desk, which calls for different recruitment methods than email or intranet messages. Employer 1: "This would mean approaching them in an entirely different way." Employer 2: "They're much more difficult to reach in terms of communication. Generally speaking, if you post a message [on the intranet], it will at least be read by the office worker, because he is on his computer anyway, checking periodically for the latest updates. However, what we hear from people in the field [...] is that they never look there." Additionally, some employers mentioned that they have low literate employees who are harder to reach using certain communication channels. For example, written messages via intranet or (digital) newsletters are found less suitable to reach low literate employees. Employer 3: "These people don't have a PC at home, nor a tablet or smartphone." "We're having to teach reading comprehension to our people, because their literacy skills aren't great. We assessed our entire production staff and the outcome was way below the national average. Why? Because we have low literacy in this company."

\section{Employers lack ideas for communication strategies}

How these employees who do not have an office job can be reached was not always clear to employers. Employer 4: "So we put up posters and got to thinking: what else can we do? [...] And often I find myself wondering: what else is there? And then I can't think of anything."

Employers are not able to contact all smokers in person

The recruitment strategy of approaching individual employees also had its barriers. For large companies, it takes more manpower to reach all smokers on an individual level. Employer 5: "Within an organization of this size, it's simply impossible to approach everyone personally."

\section{Employers lack communication skills}

Additionally, approaching employees requires skill and confidence from the supervisor. Employer 5: "I think that the ability to have a good conversation with your employee [...] not only requires the requisite skills, [...] but also timing, acceptance by the other party, sincerity almost an innate sensitivity, because an employee will know if their line manager is sitting on the other side of the table and not giving them their full attention." Supervisors can also be uncertain about their role in promoting smoking cessation. Employer 5: "What is the role of line managers in terms of sustainable employment and vitality? How do they gain the courage to broach the subject of health and vitality with the people they manage? So I don't think line managers will point employees in the direction of a smoking cessation program."

\subsubsection{Facilitators for the reach of the program}

Combining multiple communication strategies

Employers suggested that combining recruitment strategies would be most effective, such as personal recruitment through supervisors and general recruitment via email, 
intranet messages and posters. Employer 6: "My experience with the programs we've set up is that a combination of approaches works best, so you use both a written and an oral approach and you visit both the shift and the department." Various channels for (written) messages were mentioned. Employer 6: "You could use leaflets, brochures, flyers or items on the digital narrowcast screen on the shop floor."

Individual approach through team leaders

Employers believed that a good strategy to reach employees is to approach them individually through supervisors or team leaders. Line managers were considered suitable for this task, because of their personal relationship with the employees, and because they often know employees who smoke. Therefore, it was mentioned that it would be important to first gain support from the managers in order to increase reach among employees. Employer 7: "Line managers usually know who smokes and who doesn't, so they can approach people in person."

Reaching employees through word of mouth

An effective recruitment method could be if successful quitters become ambassadors and inform their colleagues about the program and convince them to participate. Word of mouth through supervisors and colleagues was successful within a company that employs many low-literate employees. Employer 7: "And at some point the word started spreading, to the point that it became a topic of conversation even in the smoking area: 'Are you going?'

Organizing an information meeting for employees

Some employers had organized an information meeting for employees prior to the start of the program. During this meeting, which was intended to lower the threshold for employees to subscribe, a smoking cessation counsellor provided information about the program. One company had ex-smokers who had participated in a previous training as ambassadors during the information meeting. Employer 13: "We invited two people who had been in the first group to the meeting to serve as ambassadors of sorts and explain how the program had benefited them. [...] Eventually, they had quit completely. They could explain very well to the group what it had been like for them [...]."

\section{Adoption: what are barriers or facilitators for the adoption of financial incentives and group trainings?}

\section{Barriers for the adoption of group trainings}

Financial benefits of smoking cessation are unclear

Another barrier that was mentioned by employers, was that the financial benefits of providing a smoking cessation training were unclear. This was because employers did not know which part of the company's absenteeism was caused by smoking, but also because the employer was uncertain whether a training would be worth the investment for temporary employees. Employer 3: "It's tough to link absenteeism to smoking, isn't it. It's not like our employees suffer from shortness of breath or things like that. [...] So no, I don't see it reflected in our absenteeism figures." 


\section{Disappointment in cessation outcomes}

Two employers who had offered a group training in their company in the past were disappointed in the quit success rate of the employees. Employer 6: "What disappointed me was that a lot of people relapsed over time, so in the long run the results were not as great as they seemed initially." However, they did not see the high relapse rate as a reason to stop offering smoking cessation trainings. In one case, the employer saw it as a reason to consider removing smoking areas from the worksite.

\section{Decision makers smoke themselves}

Several respondents mention that a barrier towards implementation is that the decision maker or another key influential person in the company smokes, and is therefore not an advocate for a smoking cessation intervention. Employer 8: "A giant obstacle to the implementation of any smoking cessation program is the fact that our production manager smokes like a chimney. He plays a key role within the organization, likes the good things in life and doesn't want anything to do with 'trivialities', including the health of employees."

\section{Barriers for the adoption of financial incentives}

Incentives are considered unfair

Some employers thought that offering a smoking cessation group training for free and also letting people participate during work time was already quite generous. Offering the trainings is defensible, according to several employers, because it is part of being a good employer to stimulate healthy behavior and it saves costs if employees are healthier. However, the incentives were more often viewed as "unfair", because non-smokers and e.g. people with obesity are not rewarded. Some employers did not find incentives unfair themselves, but worried that employees might do and would complain. Employer 9: "I can see the reaction now: 'You gifted them $€ 150$, but what about me? What do I get? I haven't smoked for years.' [...] That's something I have to deal with, as primal a response as it is. It may not be fair, but it's something that happens." Employers expressed the need to be able to justify the incentives towards others. While employers were concerned about negative reactions from non-smokers, most employers that had first-hand experience with the incentives because of their participation in the randomized trial did not report that they experienced actual resistance from employees. One employer reported receiving negative reactions from a 'handful' of people when they first introduced the intervention, while the second time the program was offered there were no complaints, and concluded that it was probably a matter of 'acceptance and habituation' for the employees.

Incentives are not consistent with peoples' values

Some employers made judgements about financial incentives that were not really grounded in arguments, but were just not consistent with their values. For example, the opinion that you should not reward people for normal behavior or for quitting with bad behavior. Also, employers mentioned that smokers already save enough money with quitting smoking and thus do not need a financial reward. Employer 1: "It's better for both the employee and his wallet. To quit smoking is its own reward, but on top of that we'll also reward him, even though he's been indulging in behavior that is hard for us to condone for many years." Finally, some employers expressed the opinion that you need to have intrinsic motivation to quit and it is not right to quit for a reward. 


\subsubsection{Facilitators for the adoption of group trainings}

Responsibility for the health of employees

Employers were more open to adopting smoking cessation trainings if they considered stimulating their employees to quit smoking part of sustainable employability, and if they thought the company had a responsibility for the health of their employees. Additionally, knowing that a large proportion of the employees were smokers was a facilitator. Employer 4: "Our 2016 Preventive Medical Examination revealed that quite a few of our people were smokers - around 33\%, I believe. [...] This was an extraordinary figure, way above [the national average]. We felt that we had an opportunity as well as a duty as an employer to do something about it."

\section{Becoming a smoke-free company is an opportunity}

Some companies were planning to make the entire worksite smoke-free in the near future. This policy change was for some employers a reason to offer a smoking cessation program, both to support employees who smoke and to make the transition to a smokefree company more acceptable to their smoking personnel. Employer 10:"As of 1 September, this should be a smoke-free environment. [...] As a consequence, we're already preparing our employees by offering smoking cessation programs."

\section{Annoyance about smoking breaks}

A frequently mentioned facilitator for the adoption of smoking cessation trainings was the annoyance from non-smoking employees that their colleagues took too many or too long smoking breaks. Employer 6: "Another problem, which really bothers the non-smokers, is that the smokers are slow to return from the smoking area. It's an oft-repeated complaint: 'I'm off for a quick smoke.' 'Sure, I'll see you in a minute.' And just like that, a minute turns into half an hour [...]."

\subsubsection{Facilitators for the adoption of financial incentives} Believing that financial incentives are effective

Some employers mentioned that scientific evidence for the effectiveness of financial incentives was important for the adoption of incentives. Employer 4: "If that survey clearly indicates that the program will yield positive results as well, then I expect our management will want to come on board." An employer suspected that financial incentives would be particularly motivating for employees with a lower income. Employer 13: "If you ask me, I think a reward policy will work even better [for low-income employees]. After all, these people are more sensitive to that sort of thing." On the other hand, an employer doubted that incentives would be a motivator for employees with a high salary. Employer 11: "As for employees on a higher pay grade, I don't think it'll make them sufficiently motivated to quit. I find that hard to believe."

Recognizing the cost-benefit of financial incentives

The costs of the financial incentives would not be a problem if the management regarded it a means to decrease the much larger costs of absenteeism. Employer 13: "If you look at what we stand to gain, it [the incentive costs] shouldn't be an issue." 
Making financial incentives fairer

Financial incentives for quitting smoking would be more acceptable to some employers if they were fairer, i.e. if not only the smokers, but all employees could earn rewards. Employer 9: "As regards people who never smoked, I don't want to give them the same reward. I want to show my appreciation in another way. [...] The bottom line is that I need to do something for that group as well." A suggestion by some employers was incentivizing other healthy behavior such as exercising or healthy eating. A second solution to the unfairness obstacle that was mentioned by some employers were team or group rewards, so that non-smoking employees could share in the reward. Employer 14: "The money has to come out of a fund and needs to be distributed equally, so everybody gets a share, including the non-smokers." Employers mentioned some examples of financial incentives that they considered fairer than vouchers or money for the individual smoker, such as vouchers for a shared healthy lunch at the workplace or for a dinner with a colleague.

\section{Implementation: how would you implement group trainings, and how would you implement incentives when they are no longer paid by a research grant?}

\section{Barriers for the implementation of group trainings and incentives}

The costs of trainings and incentives

Some employers mentioned that the costs of a smoking cessation program with incentives are so high that it could not simply be paid from an existing budget, and that they would need to make a budget available if they wanted to implement the program. Employer 1: "We'll struggle to do this on the meagre vitality budget that we have - it's simply too expensive." The intervention costs were a reason for some employers to want to offer the intervention only to permanent employees. Employer 14: "Which company would gift $a € 300$ reward to - or pay for a training program for - seasonal workers? Not us. We wouldn't see a return on our investment."

\section{Limited time and resources}

The persons who would be responsible for organizing a smoking cessation program are often occupied with many other tasks and may have other priorities. Employer 4: "We did originally intend to do a training program this year, but that put me in a tight spot: since we're also doing a PME and I'm organizing that as well, it was feeling like it was becoming a bit too much." An employer mentioned that he needed to find support from other colleagues to help him with the organization of the program. Employer 9: "If I want to get this done, I need to find people within the organization who are behind me and who will not only say, 'Oh boy, that sure sounds good, go right ahead,' but also, 'I'll give you a hand - we'll do it together."' Additionally, respondents mentioned that they have to choose between a smoking cessation intervention and other health promoting programs. Employer 2: "I'm strapped for time myself. Quitting smoking is such a minor thing - all right, it's made out to be a big thing, especially in this phase, but to spend all that time on it... Other things matter as well. That makes it really complex and complicated, so at some point you have to make choices: what do I invest in and what do I not invest in?"

\section{Facilitators for the implementation of group trainings and incentives}

Having an insurance company that reimburses the group trainings and incentives 
Some employers had a collective insurance for their employees that covered the cost of the smoking cessation trainings, which was a reason for one organization to offer their employees the training for a second time. Employer 13: "So at that point we invited [the smoking cessation provider] to deliver the training program again in 2018, also because [the smoking cessation provider] had a contract at that time with [our collective health insurance provider], so people who were insured with [our collective health insurance provider] could sign up to the program at no cost to them - or to us."

Participation of the partner of the employee

Some companies expressed the idea of involving the smoking partners of the employees and let them participate in the group trainings. Employer 2: "We've already decided that if we're going to offer support, we're also going to do things like involving the employee's life partner. [...] After all, it's hard to quit smoking if you've got a partner at home who isn't quitting."

\section{Alternatives for group trainings}

Some employers mentioned that they wanted to offer other types of smoking cessation support than group trainings, because the employees' different work schedules made it difficult to schedule group meetings, or because not every employee likes the idea of a group training. Employer 2: "I've spoken to people in the field whose attitude went something like this: 'Do you think I'm mad? I'm not going to quit as part of a group. If I'm going to quit, I'll do it on my own, but in that case I may need information or other support or maybe a one-onone conversation.' And we told them in the early going, 'Let's do it, we're there for you."'

\section{Trainings within working hours}

The opinions of the employers on whether to organize the group trainings within or after working hours were mixed. On the one hand, most employers recognized that planning the training within working hours would be the most attractive option to employees. On the other hand, some employees considered it fair that employees would invest some of their free time, since the company already paid for the trainings. Employer 1: "I feel that if you're going to quit smoking, you should be willing to invest in it."

\section{Maintenance: would you want to keep offering group trainings and incentives to your employees?}

\section{Barriers}

Smoking is not included in the existing health promotion program

A barrier for the maintenance of group trainings and financial incentives was that in most companies, unlike other health promotion themes such as exercise, healthy food and stress management, smoking cessation was not part of the company health promotion program. Employer 2: "We often talk about health, vitality, sustainable employment and things like that, but smoking hardly ever comes up." With the exception of a single company, none of the employers had a long-term plan for offering smoking cessation support to their employees. This meant that if a company had provided a smoking cessation training in the past, it was a one-time event. Additionally, the decision to organize a smoking 
cessation training depended on the priorities that the employer had at that moment in time.

Little enthusiasm for smoking cessation among employees

Some employers had the idea that there was no demand for smoking cessation trainings among their employees. Employer 12: "We still want to offer the program again, but at the same time we're seeing the same problems that made offering it so tricky the first time. [...] I believe two employees have now come up to ask, 'Will there be another program?' and that's about it. And when I ask around among people, there's not much enthusiasm for a smoking cessation program."

\section{Facilitators}

Making smoking cessation part of larger health promotion program

Several employers mentioned that smoking cessation trainings could fit within the existing health promotion program of the company. Employer 13: "The Fit program was based on the five pillars of exercise, smoking, alcohol, vitality and relaxation. Those were the five components of the vitality program that was rolled out within the organization. Obviously, smoking was one of them." Some employers mentioned that it would be easy to implement a smoking cessation program if they would choose to, because they had easy access to a vitality budget that they could use for a project of their choice. Employer 3: "Of course I'm happy to set [money] aside. I manage that budget. If you're telling me, 'Vitality is a key HR focus for this year,' it means you need to set aside money, and those funds can help."

\section{DISCUSSION}

Our qualitative interview study among employers revealed several barriers and facilitators for the reach, adoption, implementation, and maintenance of smoking cessation group trainings and for providing financial incentives for successful smoking cessation.

When examining how employees can be reached with an offer of a smoking cessation group training, it became apparent that there were barriers that seemed specific to the workplaces with employees with a low educational level. Employers mentioned that it was more difficult to reach low literate employees and that they could not reach certain employees through digital communication channels. Employers explained that they needed to use multiple communication strategies, including communication via line managers, word of mouth, and information meetings. An additional important finding was that employers perceived a lack of skills and opportunities in reaching their employees. Some did not have ideas about alternative communication strategies (besides using intranet and posters). Many employers believed that actively approaching individual employees in person was the most effective strategy to reach employees. Support for this idea is provided by several studies showing that a pro-active approach is effective to reach smokers with a low socioeconomic status. ${ }^{19,20}$ However, employers did not think it would be possible to contact all employees in person. Additionally, they lacked communication skills and confidence to bring up smoking cessation, which they considered a sensitive topic. A previous qualitative review also pointed to difficulties in the access and motivation 
of participants by the implementer as an obstacle to the implementation of health promotion interventions in the workplace. ${ }^{21}$ Our results indicate that in order to reach the target group of smoking employees with a lower level of education, it may be necessary to offer employers an action plan and develop training and support for employers in health behavior related conversational skills to increase their confidence. Interestingly, employers did not bring up the idea of using financial incentives as a strategy to recruit employees, while incentives may increase participation levels amongst employees. ${ }^{22,23}$ This may be connected to the finding that employers find it difficult to justify financial incentives to their (non-smoking) employees.

When asking employers about the barriers and facilitators for the adoption of group trainings and incentives, it was clear that most employers felt a responsibility for the health of their employees and that they considered facilitating smoking cessation part of promoting sustainable employability and being a good employer. Several crucial points were made about effectiveness, cost-effectiveness, fairness and moral values. Employers generally believed the scientific evidence showing that financial incentives can significantly increase quit rates, and some said that this proof of effectiveness would be a reason for the company management to adopt the incentives. This is in line with previous research showing that the willingness to use interventions with financial incentives increases with their effectiveness. ${ }^{24}$ In contrast, some employers that had firsthand experience with group trainings were disappointed in how many smokers returned to smoking a while after the group training had ended. This could be a sign of unrealistic expectations of the influence of a smoker's motivation to quit smoking on their actual quit success. ${ }^{25}$ More insight into the nature of tobacco addiction and the associated relapse rates may show employers the results of the intervention in a more positive light, and may encourage them to provide a recurrent smoking cessation program in which employees may participate multiple times. The cost-effectiveness of financial incentives was also generally believed, but some employers did not see immediate financial benefits of stimulating smoking cessation in their own company. Therefore, it may be important for the implementation of smoking cessation trainings with incentives that employers are provided with a compelling 'business case' which gives an overview of the costs of smoking employees ${ }^{26,27}$ and the financial benefits to their company if they invest in helping employees to quit smoking.

Fairness was a significant theme. Many employers considered incentives unfair, because only employees who smoke would receive rewards and non-smoking employees would not. From the interviews, it seemed that not so much the employers' beliefs of unfairness were a barrier for the adoption of incentives, but rather the perceived beliefs of their non-smoking employees. The employers were uncertain on how they could justify the financial incentives to their non-smoking employees, and were afraid of negative reactions. This fear of negative reactions may be unwarranted, since employers who had experience with financial incentives did not report many complaints from non-smokers. For the implementation of financial incentives, it may thus be necessary to take away these concerns. Employers also suggested that they would be more willing to implement alternative incentives that were more acceptable and less unfair. Fairer incentives were incentives from which non-smokers could also benefit, such as team rewards, or incentives 
that are available to all employees, such as incentives for different types of health behavior. An interesting note is that this last suggestion contradicts the also reported barrier that financial incentives are too expensive. Rewards in the form of vouchers for a (team) activity or event were also thought more acceptable than more freely disposable gift vouchers or money. In line with this result, previous studies have shown that shopping vouchers were more acceptable than cash or luxury items, because they were seen less as a reward and because they provide some control over how the vouchers are spent by the receiver. ${ }^{11,24,28}$ A potential pitfall for changing financial incentives into more acceptable options may be that the incentives become less attractive to employees and consequently less effective. ${ }^{29}$ An additional matter of concern regarding shared rewards that should be prevented is that a failed quit attempt may lead to negative feelings toward a smoker if because of it the non-smoking colleague does not receive the reward.

A related barrier for the adoption of financial incentives was that financial incentives for quitting smoking were often not consistent with the employer's personal values. Some interviewees had a negative attitude towards rewarding employees for a behavior that is in their self-interest, which has also been found in previous research. ${ }^{29,30}$ Additionally, some employers questioned why they would reward 'bad behavior' such as smoking. This 'moralization'31 of smoking behavior as opposed to other health-related behaviors such as exercise and dietary behavior has been suggested as a reason for the reluctance to use financial incentives for smoking cessation..$^{24}$ It will be essential for an implementation strategy to address this moralization, and help employers see financial incentives as a reward for successful behavior change and as a token of appreciation for 'good' behavior instead of 'bad'.

The main barriers for implementation of the group trainings and incentives were the resources that employers need to implement the intervention, in the form of costs, time and the deployment of staff. In their review on financial incentives for smoking cessation Notley et al. (2019) ${ }^{32}$ also discuss that the costs of financial incentives may be a main barrier from the employers' perspective. The employers that were interviewed in the current study said that the costs of trainings and incentives were a barrier, because they did not have a budget readily available for such an expensive intervention. Nevertheless, employers also mentioned that it would be possible to fund the intervention if the company management would decide to invest in promoting smoking cessation and would allocate a budget to this purpose in advance. The cost issue may lead employers to choose for incentives with a relatively low value. From the current available research, it is unclear how incentive amounts relate to effectiveness, since there is a lack of research that directly compares different incentive amounts. ${ }^{32}$ While it is unlikely that there is a linear dose-response relationship, it is probable that incentives below a certain value are less effective. ${ }^{32}$

Not only the costs were a barrier, but also the time that employers needed to organize the intervention and to find colleagues who could assist with the implementation of the trainings, while they were already occupied with many other tasks. In previous reviews, lack of financial, staffing or material resources was also identified as the most frequently reported barrier for the implementation of worksite health promotion programs. ${ }^{21,33}$ The 
perceived lack of time and staff to implement smoking cessation trainings may be a matter of prioritizing reducing smoking among employees by the higher management and allocating more resources to the implementation of smoking cessation trainings. Strong management support for the intervention, which was the most frequently reported facilitator of the implementation of workplace health promotion programs, ${ }^{33}$ could help to solve these resource issues.

Finally, maintenance of the group trainings and incentives was discussed. A key aspect was that smoking cessation was often not included in the company's health promotion program, which would help to ensure maintenance. This meant that if a company decided to offer as smoking cessation program, this was usually organized as a one-off intervention. This could be related to the barrier of having a lack of time and staff to implement the intervention; if the smoking cessation program was part of the larger company health program, resources could be reserved for the implementation and standardized procedures could be developed for the recruitment of employees and for the execution of the program. In addition, not making a smoking cessation intervention part of the company health promotion plan may enhance the risk that the intervention is not continued in the following years. This may be a reason why only one of the nine interviewed employers who had organized a smoking cessation training in the past was still offering the training. A final barrier for the maintenance of group trainings and incentives was that employers experienced little enthusiasm for smoking cessation among employees, which could be linked back to the difficulties that employers had with reaching employees.

\section{Implications}

All of the barriers and facilitators that we uncovered in our study should probably be considered to ensure optimal implementation and maintenance of smoking cessation group trainings with financial incentives in companies. We think the following actions should be prioritized in order improve the implementation process in workplaces with relatively many employees with a low educational level: (1) training and helping employers in how to reach their employees and convince them to take part in the group training, (2) convincing employers that their employees will not consider the incentives unfair and coming up with alternative incentives for employers who really want less unfair incentives, (3) explaining the cost-effectiveness of smoking cessation group trainings including financial incentives, and (4) ensuring that smoking cessation becomes a normal part of existing health promotion programs.

\section{Limitations}

Our study has certain limitations that should be acknowledged. We only interviewed employers from larger companies in the Netherlands (at least 100 employees) where relatively many employees with a low educational level work. Therefore, the results cannot be generalized to other types of companies or companies in different countries. Additionally, most of the companies where our respondents worked were in the adoption phase, and thus could comment less easily on the implementation and maintenance phase. Finally, it is also important to assess barriers and facilitators among employees with a low educational level themselves. The employers that we interviewed were often high 
educated and may not have been able to understand how their low educated employees could be stimulated to participate in smoking cessation group trainings with incentives. Therefore, significant elements from the perspective of employees may be missed.

\section{Conclusion}

Implementation of financial incentives for successful smoking cessation in the workplace will not happen automatically after research has shown its effectiveness. Strategies are needed to facilitate implementation. Our study has revealed priority actions in order to support the implementation process in workplaces with relatively many employees with a low educational level. These include developing strategies to reach employees, making financial incentives acceptable, presenting the cost-effectiveness of trainings and incentives, and making smoking cessation part of the existing workplace health promotion program. 


\section{REFERENCES}

1. Goetzel RZ, Henke RM, Tabrizi M, et al. Do workplace health promotion (wellness) programs work? J Occup Environ Med. 2014;56(9):927-934.

2. Baxter S, Sanderson K, Venn AJ, Blizzard CL, Palmer AJ. The Relationship between Return on Investment and Quality of Study Methodology in Workplace Health Promotion Programs. Am J Health Promot. 2014;28(6):347-363.

3. van den Brand FA, Nagelhout GE, Winkens B, Chavannes NH, van Schayck OCP. Effect of a workplace-based group training programme combined with financial incentives on smoking cessation: a cluster-randomised controlled trial. Lancet Public Health. 2018;3(11):e536-e544. doi:10.1016/S2468-2667(18)30185-3

4. Volpp KG, Troxel AB, Pauly MV, et al. A randomized, controlled trial of financial incentives for smoking cessation. N Engl J Med. 2009;360(7):699-709.

5. Cuellar A, Haviland AM, Richards-Shubik S, et al. Boosting workplace wellness programs with financial incentives. Am J Manag Care. 2017;23(10):604-610.

6. NBGH. Employers continue to expand well-being programs and increase financial incentives for employers. In: National Business Group on Health; 2018.

7. Willis Towers Watson. Employee Health and Business Success. Making the connections and taking action. Global research summary. 2016.

8. Glasgow RE, Vogt TM, Boles SM. Evaluating the public health impact of health promotion interventions: the RE-AIM framework. Am J Public Health. 1999;89(9):1322-1327.

9. Hulscher $M$, Wensing $M$, Grol R. Multifaceted strategies for improvement. In: Grol R, Wensing M, Eccles M, Davis D, eds. Improving patient care: the implementation of change in health care. Chichester, UK: John Wiley \& Sons, Ltd.; 2013:278-287.

10. van den Brand FA, Nagelhout GE, Winkens B, Chavannes NH, van Schayck OCP, Evers SMAA. Cost-effectiveness and cost-utility analysis of a work-place smoking cessation intervention with and without financial incentives. Addiction.2020;115(3):534-545.

11. Giles EL, Robalino S, Sniehotta FF, Adams J, McColl E. Acceptability of financial incentives for encouraging uptake of healthy behaviours: A critical review using systematic methods. Prev Med. 2015;73:145-158.

12. Halpern SD, Madison KM, Volpp KG. Patients as mercenaries?: the ethics of using financial incentives in the war on unhealthy behaviors. Circ Cardiovasc Qual Outcomes. 2009;2(5):514-516.

13. Brown T, Platt $S$, Amos A. Equity impact of European individual-level smoking cessation interventions to reduce smoking in adults: a systematic review. Eur J Public Health. 2014;24(4):551-556.

14. Hill S, Amos A, Clifford D, Platt S. Impact of tobacco control interventions on socioeconomic inequalities in smoking: review of the evidence. Tob Control. 2014;23(e2):e89-e97.

15. Springvloet L, Bommelé J, Willemsen M, Van Laar M. Kerncijfers roken 2017 [Key figures smoking 2017]. In. Utrecht: Trimbos Institute; 2018.

16. Heijndijk S, Willemsen M. Dutch tobacco control: Moving towards the right track? FCTC shadow report 2014. In: The Hague, The Netherlands: Dutch Alliance for a Smokefree Society; 2015.

17. van Mourik DJA, Willemsen MC. Netherlands: National Prevention Accord to improve health and reduce smoking [news analysis]. 2018;28:126.

18. Ritchie J, Spencer L. Qualitative data analysis for applied policy research. In: Bryman A, Burgess RG, eds. Analyzing Qualitative Data. Routledge: London, UK; 1994:173-194.

19. Haas JS, Linder JA, Park ER, et al. Proactive Tobacco Cessation Outreach to Smokers of Low Socioeconomic Status: A Randomized Clinical Trial. JAMA Internal Medicine. 2015;175(2):218-226. 
20. van Wijk EC, Landais LL, Harting J. Understanding the multitude of barriers that prevent smokers in lower socioeconomic groups from accessing smoking cessation support: A literature review. Prev Med. 2019;123:143-151.

21. Rojatz D, Merchant A, Nitsch M. Factors influencing workplace health promotion intervention: a qualitative systematic review. Health Promot Int. 2016;32(5):831-839.

22. Robroek SJW, van Lenthe FJ, van Empelen P, Burdorf A. Determinants of participation in worksite health promotion programmes: a systematic review. International Journal of Behavioral Nutrition and Physical Activity. 2009;6(1):26.

23. Cahill K, Lancaster T. Workplace interventions for smoking cessation. The Cochrane Library. 2014.

24. Promberger M, Dolan P, Marteau TM. "Pay them if it works": Discrete choice experiments on the acceptability of financial incentives to change health related behaviour. Soc Sci Med. 2012;75(12):2509-2514.

25. Hughes JR. Motivating and helping smokers to stop smoking. J Gen Intern Med. 2003;18(12):10531057.

26. Berman M, Crane R, Seiber E, Munur M. Estimating the cost of a smoking employee. Tob Control. 2014;23.

27. Troelstra SA, Coenen P, Boot CR, Harting J, Kunst AE, van der Beek AJ. Smoking and sickness absence: a systematic review and meta-analysis. Scand J Work Environ Health. 2019.

28. Bonevski B, Bryant J, Paul C. Encouraging smoking cessation among disadvantaged groups: A qualitative study of the financial aspects of cessation. Drug and Alcohol Review. 2011;30(4):411-418.

29. Volpp KG, Asch DA, Galvin R, Loewenstein G. Redesigning Employee Health Incentives Lessons from Behavioral Economics. N Engl J Med. 2011;365(5):388-390.

30. Promberger M, Brown RCH, Ashcroft RE, Marteau TM. Acceptability of financial incentives to improve health outcomes in UK and US samples. J Med Ethics. 2011;37.

31. Rozin P, Singh L. The Moralization of Cigarette Smoking in the United States. J Consum Psychol. 1999;8(3):321-337.

32. Notley C, Gentry S, Livingstone-Banks J, Bauld L, Perera R, Hartmann-Boyce J. Incentives for smoking cessation. Cochrane Database of Systematic Reviews. 2019(7).

33. Wierenga D, Engbers LH, Van Empelen P, Duijts S, Hildebrandt VH, Van Mechelen W. What is actually measured in process evaluations for worksite health promotion programs: a systematic review. BMC Public Health. 2013;13:1190. 


\section{SUPPLEMENTARY FILE}

\section{Interview Guide}

Note: Questions were slightly adapted based on whether the respondent had experience with smoking cessation group trainings with financial incentives; respondents without previous experience received questions a hypothetical situation in which group trainings and incentives would be implemented.

Background employer

- What is your age, education and position?

- What type of company do you work for? In which industry?

- What is the background of the company's employees (number of employees, level of education, number of employees with low socioeconomic status, extent of absenteeism, smoking behavior)?

- Does the company where you work have a policy on lifestyle or healthy behavior? If so, what is the content of this in brief?

- What is your responsibility in deciding whether or not to offer stop smoking training to employees? Who else is responsible?

\section{General}

- When did your company offer the stop smoking training (in combination with financial incentives)?

o How did this go (broadly)? What went well? What did not go well?

Reach

- How did the distribution of the invitation to participate in the stop smoking training (including rewards) to employees go? Which approach was used for this? How did this approach come about? What went well and less well?

- Were all employees (both executive staff and management) aware of the stop smoking training? Which employees do or do not? What is the deliberate choice to inform some employees or not? If yes, why yes / no?

- How many employees ultimately took part in the training? Which employees did or did not? Was this expected? Why not? 
Adoption

- What are the most important goals for your company when offering stop smoking training with rewards to employees (for example, healthier employees, higher productivity, less absenteeism)?

- To what extent was there support within the company for offering the stop smoking training (with rewards)? Have measures been taken to increase support (both among executive staff and management)? If yes which one? Which were particularly successful? And which less successful?

- What hindering factors are there for offering stop smoking training (with financial incentives) in this company? What disadvantages are there for the employer to offer such a training?

- What would make it easier to offer stop smoking training (with financial incentives) at this company? What are the benefits of employing such training for employers?

- What was a decisive factor for your company in choosing to offer the stop smoking training?

- What would be a deciding factor to offer financial incentives for quit success in addition to the stop smoking training?

- Do you see offering stop smoking training as a task or responsibility of the employer?

- Do you see offering a financial reward for successful stoppers as a task or responsibility of the employer?

Implementation

- To what extent has the stop smoking training been offered within your company as originally intended? What was done differently than was actually intended? Why? Which factors played a role in this?

- How would you do it if you had to pay the financial incentives as an employer yourself? Where or from whom in the organization do you expect resistance or support? What kind of resistance or support do you expect?

o What do you think is a reasonable financial reward (suitable for the person, feasible for the organization)?

o In what form would you like to give a financial reward (voucher, cash, something else)? 
Maintenance

- Is the stop smoking training (including financial incentives) currently being offered to employees within your company?

- Do you intend to continue to do this or to do so in the future? What are barriers and facilitators for this?

- What expectations do you have about the (permanent) implementation of a stop smoking training in combination with financial incentives in the future?

- What role does financing the stop smoking training and the financial incentives play in the implementation?

Smoke-free policy

- Do you or your company have intentions or plans to turn the company into a smoke-free environment? Why or why not? If so, which plans?

Remaining

- Do you have anything to add to the interview? 


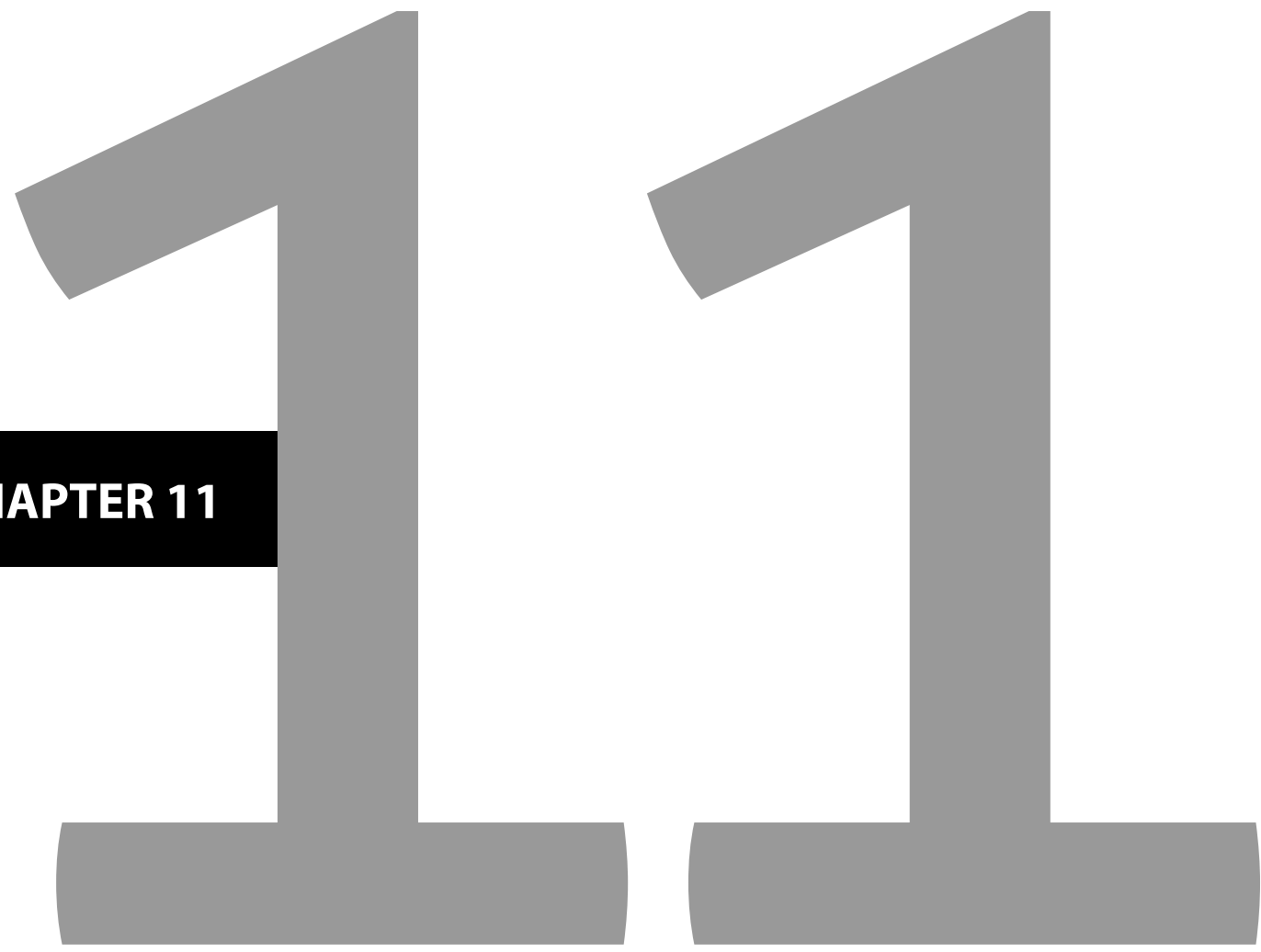
General Discussion 



\section{GENERAL DISCUSSION}

The aim of the current dissertation was to assess the effectiveness of an intervention comprising financial incentives for quit success on top of a smoking cessation group training program in a workplace setting. In this general discussion, an overview is provided of the findings of the studies in the current dissertation, followed by an in-depth view on these findings. Subsequently, methodological considerations of the studies performed are discussed, and the general discussion closes with research priorities and policy recommendations.

\section{Main findings}

In Chapter 2, a systematic review and meta-analysis shows that removing the costs of smoking cessation treatment for tobacco smokers can increase the proportion of smokers who attempt to quit, use smoking cessation treatments, and succeed in quitting. We did not find consistent evidence of an effect of financial incentives directed at healthcare providers on quit success.

Chapter 3 comprises a survey study with data from representative samples of smokers in the Netherlands and the United Kingdom. The results show that smokers who mentioned that free or lower cost smoking cessation medication is a trigger for thinking about quitting smoking are more likely to undertake a quit attempt (in the UK), and are more likely to use medication during a quit attempt (in both countries).

Chapter 4 presents the study protocol of a cluster randomized controlled trial investigating the effect of a workplace-based group smoking cessation training program combined with financial incentives on smoking cessation.

The results of the cluster randomized controlled trial in Chapter 5 demonstrate that financial incentives for smoking cessation on top of a workplace smoking cessation group training program are effective in significantly increasing the number of successful quitters up to 12 months, compared to a group training program without incentives.

Qualitative interviews with employees who had participated in the cluster-randomized trial with financial incentives, presented in Chapter 6, show that the success factors for quitting smoking are the convenience of the workplace setting, strategies learned during the smoking cessation training, peer support from colleagues, and personal motivation to quit. The financial incentives were considered a bonus but not the main reason to quit smoking.

The survey study presented in Chapter 7 reveals a negative association between having more smokers in the social environment and the quit success of the people who had participated in the workplace smoking cessation group training. The study additionally indicates a strong positive association between feeling supported by colleagues who also participated in the smoking cessation group training and quit success. 
A path analysis study exploring pathways through which financial incentives could influence quit success is presented in Chapter 8. Next to a direct pathway, the effect of financial incentives on quit success is mediated by a higher self-efficacy for quitting smoking. Additionally, financial incentives are associated with a higher likelihood of smoking cessation medication use .

In Chapter 9 a trial-based economic evaluation is performed, showing that financial incentives are cost-effective in increasing the number of successful quitters from a societal and from an employer's perspective within a 14-month period, depending on how much society or the employer is willing to pay for a quitter. Using a life-time perspective, we provide evidence that financial incentives may be a very cost-effective intervention from a long-term perspective.

Chapter 10 consists of a qualitative interview study which revealed several barriers and facilitators for the implementation of group trainings with financial incentives in a company setting from the employer's perspective. For example, barriers were the perceived unfairness of incentives toward non-smoking employees and a lack of communication skills to reach employees who smoke. Facilitators were believing that financial incentives are effective and being convinced of the cost-effectiveness of incentives.

\section{Financial incentives significantly increase smoking cessation and are effective for smokers with a low socioeconomic status}

At the beginning of the current research project, few high-quality studies had been conducted on the topic of financial incentives to promote smoking cessation. It was uncertain whether incentives had the ability to stimulate long-term quit success, how they worked in combination with intensive cessation counselling, and whether they were effective in a workplace setting. ${ }^{1}$ Evidence on the effectiveness of financial incentives has however increased in the last few years. Our cluster randomized controlled trial presented 12-month quit rates of $41 \%$ in the intervention group compared to $26 \%$ in the control group, which is a statistically significant and clinically relevant increase in quit success. Our results are in line with a Cochrane review from 2019 which included 31 RCTs (including our cluster $\mathrm{RCT}, \mathrm{N}=20,097$ ) and found a pooled relative risk of 1.49 for quitting smoking for at least six months with financial incentives compared to control conditions. ${ }^{2}$ Based on the collected evidence it can be concluded with high certainty that incentives indeed do increase long-term smoking cessation rates.

The proportion of quitters in the current study was high in comparison with similar studies that were performed in a workplace setting. ${ }^{3,4}$ The lower success rates in previous incentives research may be explained by a lack of behavioral smoking cessation support in those studies, ${ }^{3-5}$ whereas in the current research an extensive smoking cessation group training was a standard part of the intervention. Financial incentives can serve as a motivator to quit smoking but can only be (fully) effective if a person has the ability and self-efficacy to quit smoking and maintain abstinent. Previous research has demonstrated the positive effect on quit rates when people are supported by evidence-based smoking cessation counselling that provides the quitter with the necessary skills, knowledge and peer support. ${ }^{6,7}$ It has also been observed in previous research that financial incentives 
can be applied to boost cessation rates of existing treatments such as group counselling, ${ }^{3,6}$ and that the combination of these interventions may be more effective than either intervention alone.

Subgroup analyses in this research showed that financial incentives were also effective for the participants with a low education and income. Moreover, our qualitative results indicated that financial incentives may be especially motivating for people with a lower income. These findings corroborate other research indicating that financial incentives can be effective for smokers with a low socioeconomic status (SES). ${ }^{8,9}$ This is important because often smoking cessation interventions are less effective in lower socioeconomic groups and there is need for effective interventions that can reduce inequalities in smoking. ${ }^{10}$ Together with previous studies, our results suggest that financial incentives could contribute to decreasing socioeconomic differences in smoking if they are aimed specifically at this target group.

Although the 12-month quit rate in the current intervention was particularly high for a smoking cessation intervention, still many (59\%) of the participants relapsed into smoking within the follow-up year. Most participants (83\%) were successful at quitting smoking during the group training phase where they had weekly support meetings, but the relapse rates were highest in the three months that followed the end of the training program. Steep relapse curves are also seen in unassisted quit attempts, where most relapse occurs in the first week of a quit attempt and relapse probability declines over time until relapse is minimal after 100 days of continuous abstinence. ${ }^{11,12}$ In the current research, the relapse rate between six- and twelve-months follow-up was minimal. These results suggest that there may be an opportunity to increase sustained quit rates if relapse in the first few months of a quit attempt can be prevented. There may be an opportunity to improve quit rates when quit support is extended throughout this period in the form of additional group meetings or appointments with a smoking cessation counsellor. Financial incentives could additionally contribute to preventing relapse, since our results showed that the difference in the proportion of abstinent participants between the intervention and control group increased over time from 8 to 15 percent points. Fine-tuning the amount of the financial incentives and timing of delivery of the financial incentives may provide another opportunity to bridge the vulnerable period that smokers experience between the end of the smoking cessation training program and becoming a permanent ex-smoker.

\section{A group training at the workplace has many advantages}

Our research demonstrates that a smoking cessation group training with financial incentives at the workplace has many advantages. Firstly, a smoking cessation program at the workplace was considered convenient by our participants because they could attend the meetings on location during or directly after working hours, and free of cost. Secondly, offering a free smoking cessation program at the workplace has the opportunity to reach smokers that perceive barriers to searching smoking cessation counselling on their own, in particular for people with a lower socioeconomic status. ${ }^{13}$ Thirdly, the group setting of our intervention was considered enjoyable and a major contributor to quit success according to the participants themselves. The group setting provided an opportunity to 
share experiences and receive social support, but also created peer pressure to remain abstinent and not be one of the first persons to relapse. This competitive element can be a motivator for quitting smoking ${ }^{14}$ and may be one of the contributing factors to quit success that gives group trainings an advantage over individual counselling. Especially for people with a low socioeconomic status, the social support provided in a group setting may be essential, since low SES individuals in general have more smokers in their social network, ${ }^{15}$ and a lack of social support for quitting smoking, ${ }^{16,17}$ which impairs their chance of quitting smoking successfully. ${ }^{18-20}$

It is valuable to gain more insight into the group dynamics of a smoking cessation training, as it can guide composition of effective groups. It is conceivable that the success of the entire group is influenced by the success or failure of individuals within this group. Further research is warranted to conclude whether participants who relapse into smoking should keep attending the group meetings or that they would negatively influence the other members' chances of quitting. Additionally, it may make a difference whether group members are acquaintances or even friends, for example in the case of co-workers, or strangers. In our study, participants were enrolled in the group training with colleagues, whom they were sometimes but not necessarily acquainted with before the start of the training. According to participants in our study, a group feeling developed independent of being previously acquainted as a result of their common goal to quit smoking.

\section{Financial incentives can have a direct and mediated effect on quit success}

Although the present research clearly indicates a positive effect of incentives on quit success, the smokers in our study considered the financial incentives only as an added bonus but not a reason to quit Intrinsic motivation was said to be the main reason for quitting smoking. This intriguing contradiction has been observed before in previous studies. ${ }^{21,22}$ We performed a path analysis to get more insight into how financial incentives could lead to quit success, which revealed that financial incentive might indirectly influence quit success through different mediators. We found that incentives were associated with a higher use of smoking cessation medications. This is in line with other research demonstrating that financial incentives can stimulate smokers to make use of smoking cessation treatment services, ${ }^{23}$ online quit support, ${ }^{5}$ and telephone quit line support. ${ }^{24}$ This suggests that smoking cessation interventions with incentives should also contain easy access to smoking cessation medications.

Our analysis also found that financial incentives were associated with a higher self-efficacy to quit smoking. This is an interesting new finding that should be further explored in future research, since self-efficacy has shown to be an important predictor of behavioural change. The prospect of earning the reward may have stimulated visualization of success which can increase self-efficacy. ${ }^{25}$

Previous studies have shown that the effect of financial incentives on motivating behavior change could differ between individuals, ${ }^{22}$ and may be influenced by the extent to which the individual is motivated by the particular incentive $e^{26,27}$ or by personal sensitivity to rewards in general. ${ }^{28,29}$ However, in our study we found that individual reward responsiveness did not influence the association between financial incentives and quit 
success. This topic warrants further investigation as it might inform the design of effective incentives for different subgroups of smokers.

\section{The cost-effectiveness of financial incentives}

Employers have a good reason to promote smoking cessation: it improves the health and quality of life of their employees, and it decreases smoking-related costs. The cost associated with smoking among employees is caused not just by absenteeism, i.e. absence from work due to illness or premature death related to smoking, ${ }^{30,31}$ but also by productivity losses due to presenteeism, i.e. reduced performance at work due to smoking tobacco. Smokers addicted to tobacco may experience withdrawal symptoms within 30 minutes after finishing their last cigarette, which causes loss of concentration and feelings of restlessness. ${ }^{32}$ Another cause of productivity loss are smoking breaks, although this can vary a lot depending on company policy. Some studies provide estimations of the costs related to smoking among employees. A meta-analysis focusing on working populations found that smoking was associated with a 31\% increase in risk of absenteeism and 2.9 more sickness absence days per year compared to non-smoking. ${ }^{33}$ A study that made an estimation of the average excess cost of a smoking employee over a non-smoking employee for an employer in the United States, found an annual cost of $\$ 5,816$ to employ a smoker. ${ }^{32}$ Based on Dutch absenteeism statistics, ${ }^{34}$ with average hourly wage cost of $€ 34$, it can be estimated that the annual costs of a smoking employee are around $€ 5,197$ in total, which is a sum of $€ 760$ due to absenteeism, $€ 612$ due to presenteeism, and $€ 3,825$ due to smoking breaks ( $2 \times 15$ minutes a day). On the positive side, employees who quit smoking show a decline in absenteeism within several years ${ }^{31}$ and an increase in productivity. ${ }^{31}$

The decision for an employer whether to implement an intervention often depends on the justification of the financial investment. This can be assessed in an economic evaluation that balances the costs of an intervention with its effects on the main health outcome and quality of life. Yet, barely any research has been done on the cost-effectiveness of financial incentives for smoking cessation. At the start of the current research project, no study had yet performed a full economic evaluation of financial incentives alongside a trial with a population of smokers in a workplace setting. However, financial incentives for pregnant women were cost-effective ${ }^{35}$ and providing quit line callers with modest financial incentives was a cost-effective method to increase cessation rates over a 10-year horizon. ${ }^{36}$ Our economic evaluation demonstrated that within the 14-month timeframe of the intervention, financial incentives may be cost-effective from a societal and from an employer's perspective, depending on the willingness to pay (WTP) for a quitter. Incremental cost-effectiveness ratios (ICERs) were $€ 11,546$ and $€ 5,686$ from the perspective of society and the employer, respectively. However, the timeframe of 14 months was probably too short for the occurrence of health differences between the intervention and control group. The results based on a lifetime perspective showed that incentives could be highly cost-effective with an ICER of $€ 1,249$ per quality adjusted life year (QALY), far below the Dutch WTP threshold of $€ 20,000$ per QALY. ${ }^{37}$

Conclusions from our research contribute to the evidence on the cost-effectiveness of financial incentives, improves the acceptability of incentives, ${ }^{38,39}$ and may help convince policy makers and employers to stimulate the implementation of financial incentives in 
workplaces ${ }^{40}$ Employers in the current research believed that financial incentives could be cost-effective. However, they generally were not aware of the costs of employee smoking or of the benefits if more employees quit smoking. Employers may be more inclined to implement an incentives-based smoking cessation intervention if they are provided with a business case which includes an overview of costs and benefits of offering a smoking cessation intervention for their specific company. ${ }^{32,33}$

\section{Employers perceive several barriers and facilitators for the implementation of group trainings with incentives}

Employers were found to experience several barriers and facilitators for the implementation of workplace smoking cessation group trainings with financial incentives. Facilitators that we identified comprised feeling a responsibility for stimulating a healthy lifestyle among employees, aspiring to become a smoke-free company, and believing that financial incentives are effective. Another important facilitator for the implementation of group trainings with incentives was considering the intervention a good investment that would lead to cost savings.

A major barrier was that employers find it difficult to reach employees, especially employees with a lower education level who do not work behind a desk. This difficulty with reaching employees was reflected by the relatively small number of employees that participated in the group training in each company, even in very large organizations. The group size was only ten participants on average in our cluster RCT, while 16 participants were allowed per group and companies were allowed to participate with more than one group in the study. A facilitator found to increase reach among employees was approaching them proactively and in person by team leaders, which is supported by the literature. ${ }^{13,41,42}$ However, employers were insecure on how to speak to their employees about smoking cessation as they considered it a sensitive topic. Therefore, it may be needed to develop a training aimed at employers to teach them health behavior-related conversational skills.

The second major barrier was the perceived unfairness of the financial incentives, since the incentives could only be earned by employees who smoked. Employers were particularly concerned that their nonsmoking employees would react negatively on the incentives, and they were uncertain on how they could respond to objections and justify the use of the incentives. Thus, it may be necessary to provide employers with a strategy on how to explain to their employees why they chose financial incentives as an intervention to decrease smoking and offer a set of possible responses. Some employers wanted a 'fairer' type of incentive, such as team rewards that could be shared with nonsmoking employees. Incentives in the form of a celebratory activity (such as a dinner) or aimed at stimulating healthy behavior (gym credit) were also considered more acceptable than a freely disposable gift voucher or money. This could be explained because in that way the employer has some control over how the money is spent by the employee. ${ }_{38,40,43}$ Changing the financial incentives to more acceptable versions may stimulate the implementation. However: this has the risk of strongly reducing the effectiveness of the incentives. It is therefore important to explain employers that incentives should be attractive to employees to motivate them to stop smoking. ${ }^{44}$ 
A final barrier for the implementation and maintenance of group trainings with financial incentives is that most employers have not incorporated smoking cessation in their company health program. Instead, the smoking cessation intervention was mostly organized as a one-off intervention, and most of the companies did not continue to offer a smoking cessation program despite having positive experiences with it. The lack of continuation of the program seemed a result of not prioritizing smoking cessation by the company management, which resulted in not allocating enough time or resources to organize the intervention. An implementation strategy should be developed that aims to increase employers' willingness to prioritize employee smoking cessation and to make the necessary policy changes for a sustained and well-organized implementation of group trainings with incentives.

Based on all the barriers for the implementation of financial incentives that were identified in the current research, it is unlikely that group trainings with financial incentives will automatically be implemented on a wide scale within companies in the Netherlands. It will be necessary to develop an implementation strategy to convince employers to adopt the intervention and include it in their company policy. Additionally, employers need to be supported with strategies to reach and motivate their employees to participate in a smoking cessation intervention.

\section{Methodological considerations}

The studies in the current dissertation have some limitations that should be considered. The design of the cluster randomized trial may have led to a selection of participants which could affect the generalizability of the results. The self-selection of participants means that participants in our study wanted to quit smoking and needed support to quit. The employees volunteered to participate in an extensive smoking cessation program while they were not yet certain that they would be in the intervention group and thus able to earn the financial incentives. This means that they were most likely already intrinsically motivated to quit smoking. Guaranteed incentives in a real-life situation may attract also smokers who are extrinsically motivated to quit smoking, which may lead to lower success rates compared to the ones found in the current trial. In addition, only a small number of employees in each company participated in the trial, and it is likely that particularly people who were ready and motivated to quit signed up.

Another limitation was that the reward schedule was designed such that the final biochemical measurements to verify abstinence coincided with the last financial reward. As a result, based on the current trial, we cannot be certain that the effect on smoking abstinence continued after the incentives were removed. However, other studies which did have follow-up measurements of smoking abstinence after discontinuation of financial incentives did find a sustained effect on abstinence..$^{3,4}$ Moreover, a meta-analysis found comparable effect sizes between trials with incentives coinciding with the latest follow-up and trials with follow-up after discontinuation of incentives. ${ }^{2}$ This evidence suggests that incentives have a sustained effect on smoking cessation, even after they have finished. This is supported by the relapse-curve for smoking cessation, which shows high relapse 
rates in the early phase of quitting that decrease over time and are very low for people who have sustained 12 months of abstinence. ${ }^{11}$ The financial incentives may thus help to maintain abstinence during the difficult period of the quit attempt and are no longer needed when a person has adapted to being a non-smoker. Thereby, financial incentives can have a significant long-term effect on smoking cessation even after the incentives have ended.

In smoking cessation trials, it is recommended to verify the self-reported abstinence of participants. While there is not an objective way to verify smoking abstinence over 12 months, various methods exist to biochemically detect recent smoking, all with specific advantages and disadvantages. ${ }^{45}$ In our trial, an expired-air carbon monoxide (CO) measurement was used to validate abstinence from smoking. The advantage of CO measurement is that it is noninvasive, easy to perform, and directly displays the outcome. In addition, $\mathrm{CO}$ measurement can distinguish between smoking and the use of nicotine replacement therapy or electronic cigarettes. A disadvantage of $\mathrm{CO}$ measurement is its short detection period. ${ }^{46}$ As in the current research the participants were aware when they would be tested for smoking abstinence, this could raise the concern of 'gaming', where participants abstain from smoking for a couple of days prior to the assessment in order to receive the incentives. While gaming cannot be ruled out completely, we believe that its occurrence in our study was low due to the social control of colleagues. Additionally, a previous study with incentives for smoking cessation that investigated potential gaming by comparing self-reported smoking status validated by $\mathrm{CO}$-measurements with salivary cotinine levels found in only $4 \%$ of the participants a false report of smoking status. ${ }^{47}$

Other methodological considerations are that some of the studies relied on self-reported data from questionnaires, which can be unreliable as a result of socially desirable answers, for example regarding daily number of cigarettes smoked, and may suffer from recall bias, for example when participants were asked to report their medical consumption. ${ }^{48}$ Additionally, some studies (chapter 7 and 8) were post-hoc analyses in which the groups were not randomized. Therefore, the evidence consists of associations and we cannot make claims about causality. Finally, the qualitative interviews that were conducted provided valuable insights into the personal experiences of the participants but are not suitable to explain causal relationships between certain characteristics of the intervention and successful quitting, such as the financial incentives. The reason for this is that people may not be fully aware of what motivates their behavior, as was demonstrated in this thesis, when the participants did not consider the incentives effective while the RCT proved that incentives did substantially increase quit rates.

\section{Priorities for future research}

While a body of high-quality evidence shows the effectiveness of financial incentives, there are some follow-up questions that need to be addressed to specify the most effective financial rewards and to promote the implementation of financial incentives. More research is needed on the requirements for financial incentives to be both effective in stimulating an initial quit attempt, and in decreasing the steep relapse rates that are typically seen in 
smoking cessation. ${ }^{49,50}$ There is a lack of research directly comparing different incentive amounts. The available studies have used a wide range of incentive values, and together their evidence suggests that there is not a linear dose-response relationship between the value of financial incentives and their effectiveness; even low- to-moderate-value incentives can stimulate people to quit smoking. ${ }^{2}$ This knowledge is relevant to increase the cost-effectiveness of financial incentives and to improve their affordability for employers. Moreover, more insight is needed into the schedule in which incentives should be distributed. For example, no conclusion can be made on effectivity of a high reward in the initial weeks of the quit attempt (when it is difficult to remain abstinent) versus increased amount of the incentive further along the quit attempt (to compensate for delay discounting, the phenomenon that the subjective value of a reward declines with delay to its receipt). In addition, under the premises of a fixed reward amount, it is unknown whether a couple of large rewards are more effective than frequent smaller incentives. In the current study, gift vouchers were used as a financial incentive, but it is unclear in what way the effectiveness depends on the type of reward. Since employers may be more likely to implement financial incentives when they can choose their own kind of reward, ${ }^{51}$ it should be investigated whether alternative rewards, such as products or activities, are equally effective. This also applies to the effectiveness of team rewards, which may be more acceptable to employers, less costly, and may have a different effectiveness than individual rewards.

Another research priority is to investigate extension of financial incentives for smoking cessation to other settings than the workplace. This is necessary to reach a larger population and to make the intervention available for people who are unemployed or who work in small businesses where a group training is not feasible. It is needed to assess how the concept of a smoking cessation group training with financial incentives can be made accessible particularly for low SES and disadvantaged smokers. This group encounters many barriers to finding smoking cessation support. Therefore, specific adaptations to the current intervention may be required. For example, the smoking cessation training should be organized in the close living environment such as a community center, participants should be proactively an personally recruited to participate, and participants may need additional training sessions and more intensive contact with the smoking cessation coach in between sessions. ${ }^{13}$ Additionally, it is useful to evaluate whether incentives are effective in healthcare settings where cessation treatment is provided, such as general practice care where people receive individual smoking cessation treatment from their general practitioner or practice nurse. As about three quarters of the smokers above 18 years old visit their general practitioner at least once a year, ${ }^{52}$ this setting has the potential to reach a large proportion of the national population of smokers. However, for financial incentives to be implemented in a healthcare setting, health insurance companies will most likely need to cover their costs, and the implementation thus depends on the insurers' willingness to reimburse incentives. It should also be assessed whether it is effective to combine financial incentives with e-health or $\mathrm{m}$-(mobile) health smoking cessation interventions, which can be a cost-effective addition to regular healthcare. ${ }^{53}$

A final research priority is to expand the research on the cost-effectiveness of financial incentives, which is scarce. More evidence on the long-term cost-effectiveness 
in combination with various forms of smoking cessation treatment could help to determine which amounts of financial incentives should be implemented. Evidence on the cost-effectiveness of incentives could also motivate policy makers to include financial incentives in revised treatment guidelines and may stimulate health insurance companies to include incentives in their (corporate) healthcare packages. Furthermore, it is impossible for individual employers to distinguish which part of the absenteeism in their organization can be attributed to smoking. Research using aggregated data can make employers aware of smoking-related costs and can motivate them to make smoking cessation a higher priority. There is need of a cost-tool in which employers can easily calculate the costs and benefits of providing their employees with a smoking cessation training program in combination with financial incentives. Finally, in order to promote the implementation of interventions for smoking cessation on a societal level, it is needed to decide on the monetary worth of a successful quitter. Determining the willingness to pay for an additional quitter is necessary to decide which interventions are cost-effective in stimulating smoking cessation and are thus worth the investment.

\section{Policy recommendations}

In the Netherlands, the National Prevention Agreement was published in $2018,{ }^{54}$ which has the ambition to reduce smoking below $5 \%$ in the adult population and $0 \%$ in youth and pregnant women by 2040 . To achieve this goal, the implementation of extensive tobacco control measures is essential. The goals of the Agreement can only be reached by a combination of employer support, public smoking restriction, price increase, and mass media campaigns.

It is the aim of the Prevention Agreement to stimulate employers to take up a more active role in encouraging their employees to quit smoking. The Agreement also expresses the ambition that all workplaces will be smokefree by $2040 . .^{54}$ In the last couple of years, more employers have decided to remove smoking facilities from their buildings and make the company grounds a smoke-free zone. It is important that all companies will be stimulated to implement this policy soon. However, a smoke-free company does not guarantee employee quit success. A smoke-free policy implementation should always be accompanied by smoking cessation support for employees. National policies should be developed to give employers both the opportunity and responsibility of providing smoking cessation support as a standard and continual part of their company health plan. If smoke-free workplaces in combination with accessible workplace smoking cessation support are successfully implemented, a major part of the Dutch population of smokers can be reached.

The implementation of smoking cessation group trainings with financial incentives in companies on its own will not be enough to decrease socioeconomic inequalities in smoking and reduce the prevalence of smoking in society to below $5 \%$. Public smoking restrictions need to be extended, for example to parks, terraces, and shopping districts to increase the de-normalization of smoking ${ }^{55}$ and to ease abstinence. Furthermore, the price of cigarettes should be increased substantially, because cigarette price increases 
are considered one of the most (cost-)effective policies to reduce smoking and to reduce socioeconomic differences in smoking. ${ }^{56,57}$

Until 2019, smoking cessation treatment could be reimbursed once every year by the Dutch health insurance system, but there was still a cost barrier since reimbursement applied only after people had spent a mandatory deductible for general healthcare costs (at least 385 euro in 2019). Since January 2020, all health insurance companies in the Netherlands reimburse smoking cessation treatment without a mandatory deductible. This is an important development, as removing financial barriers increases the use of evidencebased treatment, which enhances the number of successful quitters. ${ }^{58} \mathrm{It}$ is recommended to communicate through mass media campaigns that smoking cessation support is fully reimbursed by all insurance companies and to inform people on where they can receive support. This combination of treatment reimbursement with mass media campaigns has previously been very successful at increasing quit attempts in the Netherlands..$^{59}$ Moreover, campaigns should increase the public's awareness that smoking is an addiction and not just a choice behavior, because the idea that smokers can easily choose not to smoke leads to moralizing and stigmatization. Changing the view on smoking is essential, since it determines to what extent society is willing to invest in smoking cessation treatment and to accept innovative interventions such as financial incentives for quit success. In addition, stigmatization of smoking leads to people feeling ashamed for not being able to quit smoking, which may prevent smokers from seeking effective treatment. ${ }^{60,61}$

Other barriers that may prevent smokers, and in particular people with a lower socioeconomic status, from accessing smoking cessation support are limited availability of treatment opportunities in the living area, difficulties with transportation, and lack of proactive and personal advice to quit smoking from healthcare professionals. ${ }^{13,56}$ For that reason, accessible treatment options should be provided by increasing the available smoking specialist care, for example in general practice. Moreover, the guideline for the treatment of tobacco addiction (in Dutch: Zorgstandaard Tabaksverslaving) advocates that patients should always receive advice to quit smoking, even when they come for a non-related consultation. ${ }^{62}$ Implementation and adhering to this guideline should be made a priority among general practitioners and other healthcare providers. 


\section{REFERENCES}

1. Cahill K, Hartmann-Boyce J, Perera R. Incentives for smoking cessation. The Cochrane database of systematic reviews. 2015(5):CD004307.

2. Notley C, Gentry S, Livingstone-Banks J, Bauld L, Perera R, Hartmann-Boyce J. Incentives for smoking cessation. Cochrane Database of Systematic Reviews. 2019(7).

3. Volpp KG, Troxel AB, Pauly MV, et al. A Randomized, Controlled Trial of Financial Incentives for Smoking Cessation. N Engl J Med. 2009;360(7):699-709.

4. Halpern SD, Harhay MO, Saulsgiver K, Brophy C, Troxel AB, Volpp KG. A Pragmatic Trial of E-Cigarettes, Incentives, and Drugs for Smoking Cessation. N Engl J Med. 2018.

5. Etter J-F, Schmid F. Effects of Large Financial Incentives for Long-Term Smoking CessationA Randomized Trial. J Am Coll Cardiol. 2016;68(8):777-785.

6. Stead LF, Carroll AJ, Lancaster T. Group behaviour therapy programmes for smoking cessation. Cochrane Database Syst Rev. 2017(3).

7. Lancaster T, Stead LF. Individual behavioural counselling for smoking cessation. Cochrane Database of Systematic Reviews. 2017(3).

8. Mundt MP, Baker TB, Fraser DL, Smith SS, Piper ME, Fiore MC. Paying Low-Income Smokers to Quit? The Cost-Effectiveness of Incentivizing Tobacco Quit Line Engagement for Medicaid Recipients Who Smoke. Value Health. 2019;22(2):177-184.

9. Haff N, Patel MS, Lim R, et al. The Role of Behavioral Economic Incentive Design and Demographic Characteristics in Financial Incentive-Based Approaches to Changing Health Behaviors: A MetaAnalysis. Am J Health Promot. 2015;29(5):314-323.

10. Hill S, Amos A, Clifford D, Platt S. Impact of tobacco control interventions on socioeconomic inequalities in smoking: review of the evidence. Tob Control. 2014;23(e2):e89-e97.

11. Hughes JR, Keely J, Naud S. Shape of the relapse curve and long-term abstinence among untreated smokers. Addiction. 2004;99(1):29-38.

12. Kirshenbaum AP, Olsen DM, Bickel WK. A quantitative review of the ubiquitous relapse curve. $J$ Subst Abuse Treat. 2009;36(1):8-17.

13. van Wijk EC, Landais LL, Harting J. Understanding the multitude of barriers that prevent smokers in lower socioeconomic groups from accessing smoking cessation support: A literature review. Prev Med. 2019;123:143-151.

14. Bottorff JL, Oliffe JL, Sarbit G, Sharp P, Kelly MT. Smoke-Free Men: Competing and Connecting to Quit. Am J Health Promot. 2018;32(1):135-142.

15. Christakis NA, Fowler JH. The collective dynamics of smoking in a large social network. N Engl J Med. 2008;358(21):2249-2258.

16. Westmaas JL, Bontemps-Jones J, Bauer JE. Social support in smoking cessation: Reconciling theory and evidence. Nicotine \& Tobacco Research. 2010;12(7):695-707.

17. vanDellen MR, Boyd SM, Ranby KW, MacKillop J, Lipkus IMJ. Willingness to provide support for a quit attempt: A study of partners of smokers. J Health Psychol. 2016;21(9):1840-1849.

18. Hitchman SC, Fong GT, Zanna MP, Thrasher JF, Laux FL. The relation between number of smoking friends, and quit intentions, attempts, and success: findings from the International Tobacco Control (ITC) Four Country Survey. Psychol Addict Behav. 2014;28(4):1144-1152.

19. Lawhon D, Humfleet GL, Hall SM, Munoz RF, Reus VI. Longitudinal analysis of abstinence-specific social support and smoking cessation. Health Psychol. 2009;28(4):465-472.

20. Margolis R, Wright L. Better off alone than with a smoker: the influence of partner's smoking behavior in later life J Gerontol B Pscyhol Sci Soc Sci. 2016;74(4):687-697. 
21. Ormston R, van der Pol M, Ludbrook A, McConville $S$, Amos A. quit4u: the effectiveness of combining behavioural support, pharmacotherapy and financial incentives to support smoking cessation. Health Educ Res. 2015;30(1):121-133.

22. Kim A, Kamyab K, Zhu J, Volpp K. Why are Financial Incentives not Effective at Influencing Some Smokers to Quit? Results of a Process Evaluation of a Worksite Trial Assessing the Efficacy of Financial Incentives for Smoking Cessation. J Occup Environ Med. 2011;53(1):62-67.

23. Mantzari E, Vogt F, Marteau TM. The effectiveness of financial incentives for smoking cessation during pregnancy: is it from being paid or from the extra aid? BMC Pregnancy Childbirth. 2012;12:24.

24. Fraser DL, Fiore MC, Kobinsky K, et al. A Randomized Trial of Incentives for Smoking Treatment in Medicaid Members. Am J Prev Med. 2017;53(6):754-763.

25. Bandura A. Self-efficacy: toward a unifying theory of behavioral change. Psychol Rev. 1977;84(2):191-215.

26. Van den Brand FA, Dohmen LME, Van Schayck CP, Nagelhout GE. 'Secretly, it's a competition': a qualitative study investigating what helped employees quit smoking during a workplace smoking cessation group training programme with incentives. BMC Open. 2018;8(11).

27. Alexander AC, Hébert ET, Businelle MS, Kendzor DE. Greater perceived importance of earning abstinence-contingent incentives is associated with smoking cessation among socioeconomically disadvantaged adults. Addict Behav. 2019;95:202-205.

28. Martin LE, Cox LS, Brooks WM, Savage CR. Winning and losing: differences in reward and punishment sensitivity between smokers and nonsmokers. Brain Behav. 2014;4(6):915-924.

29. Nestor LJ, McCabe E, Jones J, Clancy L, Garavan H. Smokers and ex-smokers have shared differences in the neural substrates for potential monetary gains and losses. Addict Biol. 2016:n/a-n/a.

30. Weng SF, Ali S, Leonardi-Bee J. Smoking and absence from work: systematic review and metaanalysis of occupational studies. Addiction. 2013;108(2):307-319.

31. Halpern MT, Shikiar R, Rentz AM, Khan ZM. Impact of smoking status on workplace absenteeism and productivity. Tob Control. 2001;10(3):233-238.

32. Berman M, Crane R, Seiber E, Munur M. Estimating the cost of a smoking employee. Tob Control. 2014;23.

33. Troelstra SA, Coenen P, Boot CR, Harting J, Kunst AE, van der Beek AJ. Smoking and sickness absence: a systematic review and meta-analysis. Scand J Work Environ Health. 2019.

34. CBS. Ziekteverzuim volgens werknemers; geslacht en leeftijd. 2017.

35. Boyd KA, Briggs AH, Bauld L, Sinclair L, Tappin D. Are financial incentives cost-effective to support smoking cessation during pregnancy? Addiction. 2016;111(2):360-370.

36. Sung H-Y, Penko J, Cummins SE, et al. Economic Impact of Financial Incentives and Mailing Nicotine Patches to Help Medicaid Smokers Quit Smoking: A Cost-Benefit Analysis. Am J Prev Med. 2018;55(6, Supplement 2):S148-S158.

37. Zwaap J, Knies S, Van der Meijden C, Staal P, Van der Heiden L. Kosteneffectiviteit in de praktijk. Zorginstituut Nederland, 2015. 2015.

38. Promberger M, Dolan P, Marteau TM. "Pay them if it works": Discrete choice experiments on the acceptability of financial incentives to change health related behaviour. Soc Sci Med. 2012;75(12):2509-2514.

39. Giles EL, Sniehotta FF, McColl E, Adams J. Acceptability of financial incentives for health behaviour change to public health policymakers: a qualitative study. BMC Public Health. 2016;16(1):989. 
40. Giles EL, Robalino S, Sniehotta FF, Adams J, McColl E. Acceptability of financial incentives for encouraging uptake of healthy behaviours: A critical review using systematic methods. Prev Med. 2015;73:145-158.

41. Haas JS, Linder JA, Park ER, et al. Proactive Tobacco Cessation Outreach to Smokers of Low Socioeconomic Status: A Randomized Clinical Trial. JAMA Internal Medicine. 2015;175(2):218226.

42. Marcano Belisario JS, Bruggeling MN, Gunn LH, Brusamento S, Car J. Interventions for recruiting smokers into cessation programmes. Cochrane Database of Systematic Reviews. 2012(12).

43. Bonevski B, Bryant J, Paul C. Encouraging smoking cessation among disadvantaged groups: A qualitative study of the financial aspects of cessation. Drug and Alcohol Review. 2011;30(4):411418.

44. Volpp KG, Asch DA, Galvin R, Loewenstein G. Redesigning Employee Health Incentives Lessons from Behavioral Economics. N Engl J Med. 2011;365(5):388-390.

45. West RPLJ. Outcome criteria in smoking cessation trials: proposal for a common standard. Addiction. 2005;100.

46. Perkins KA, Karelitz JL, Jao NC. Optimal Carbon Monoxide Criteria to Confirm 24-hr Smoking Abstinence. Nicotine \& Tobacco Research. 2012;15(5):978-982.

47. lerfino D, Mantzari E, Hirst J, Jones T, Aveyard P, Marteau TM. Financial incentives for smoking cessation in pregnancy: a single-arm intervention study assessing cessation and gaming. Addiction. 2015;110(4):680-688.

48. Brusco NK, Watts JJ. Empirical evidence of recall bias for primary health care visits. BMC Health Serv Res. 2015;15:381.

49. Higgins ST, Washio Y, Lopez AA, et al. Examining two different schedules of financial incentives for smoking cessation among pregnant women. Prev Med. 2014;68:51-57.

50. Donatelle R, Hudson D, Dobie S, Goodall A, Hunsberger M, Oswald K. Incentives in smoking cessation: status of the field and implications for research and practice with pregnant smokers. Nicotine \& tobacco research : official journal of the Society for Research on Nicotine and Tobacco. 2004;6 Suppl 2:S163-179.

51. van den Brand FA, Magnée T, de Haan-Bouma L, et al. Implementation of Financial Incentives for Successful Smoking Cessation in Real-Life Company Settings: A Qualitative Needs Assessment among Employers. Int J Environ Res Public Health. 2019;16(24):5135.

52. Springvloet L, Van Laar M. Roken onder volwassenen: kerncijfers 2016. Trimbos Institute. https:// www.trimbos.nl/docs/a264fcf9-a3e5-44c2-9ba6-e73cebd5d2ae.pdf. Published 2017. Accessed 06-02-2020, 2020.

53. Cheung KL, Wijnen BF, Hiligsmann $M$, et al. Is it cost-effective to provide internet-based interventions to complement the current provision of smoking cessation services in the Netherlands? An analysis based on the EQUIPTMOD. Addiction. 2018;113:87-95.

54. Ministerie van Volksgezondheid Welzijn en Sport. Nationaal preventieakkoord. Een gezonder Nederland. In:2018.

55. Hummel K, Willemsen MC, de Vries H, Monshouwer K, Nagelhout GE. Social Acceptance of Smoking Restrictions During 10 Years of Policy Implementation, Reversal, and Reenactment in the Netherlands: Findings From a National Population Survey. Nicotine \& Tobacco Research. 2016;19(2):231-238.

56. Brown T, Platt $\mathrm{S}$, Amos A. Equity impact of European individual-level smoking cessation interventions to reduce smoking in adults: a systematic review. EurJPublic Health. 2014;24(4):551556. 
57. de Kinderen RJA, Wijnen BFM, Evers SMAA, Hiligsmann M, Paulus ATG. Social cost-benefit analysis of tobacco control policies in the Netherlands. Universitaire Pers Maastricht; 20162016. 9789461593429.

58. Kaper J, Wagena EJ, Willemsen MC, Van Schayck CP. Reimbursement for smoking cessation treatment may double the abstinence rate: results of a randomized trial. Addiction. 2005;100(7):1012-1020.

59. Nagelhout GE, Willemsen MC, van den Putte B, de Vries $H$, Willems RA, Segaar D. Effectiveness of a national reimbursement policy and accompanying media attention on use of cessation treatment and on smoking cessation: a real-world study in the Netherlands. Tob Control. 2014.

60. Bell K, Salmon A, Bowers M, Bell J, McCullough L. Smoking, stigma and tobacco'denormalization': Further reflections on the use of stigma as a public health tool. A commentary on Social Science \& Medicine's Stigma, Prejudice, Discrimination and Health Special Issue (67: 3). Soc Sci Med. 2010;70(6):795-799.

61. Steinberg ML. Harms and Benefits of Stigmatizing Smoking. Nicotine \& Tobacco Research. 2017;20(3):275-275.

62. Chavannes NH, Meijer E, Wind L, van de Graaf RC, Rietbergen C, Croes E. Herziene richtlijn 'Behandeling van tabaksverslaving en stoppen met roken ondersteuning'. Ned Tijdschr Geneeskd. 2017;161. 

Valorization 



\section{VALORIZATION}

The aim of this addendum is to describe the societal relevance and impact on society of the current thesis. During the CAPHRI Research Day in 28 November 2019, our research project was the winner of the CAPHRI Societal Relevance Award. The award was granted to our research team because our scientific work has brought about a change that can be seen and felt outside the academic world.

This addendum starts with a description of the societal relevance of applying financial incentives to increase smoking cessation, and subsequently explains why this thesis is relevant for certain target groups. Next, the activities are presented that were conducted to disseminate the findings of the current thesis and products that were developed based on the research findings. After this, the innovativeness of the current research is discussed, and the addendum closes with a paragraph that describes the planning of a follow-up research project that aims to study the implementation of financial incentives for smoking cessation in Dutch companies.

\section{Relevance}

Smoking is the main preventable cause of death worldwide.' The World Health Organization estimated that globally, $12 \%$ of deaths among adults aged 30 years and over can be attributed to tobacco. ${ }^{2}$ Smoking is responsible for deaths from cardiovascular diseases, cancer, and respiratory diseases. ${ }^{2}$ In Western countries, smoking is more prevalent among people with a lower income and education. This makes smoking the largest contributor to socioeconomic differences in health and mortality.,4

To reduce smoking prevalence in society and decrease this socioeconomic gap in smoking, it is therefore necessary to find new approaches that are effective in helping people with a lower education and income to stop smoking. ${ }^{5}$ The current thesis provides evidence on such a new approach; it shows the potential of financial incentives in combination with a group smoking cessation training at the workplace to stimulate smoking cessation.

Our study showed that adding a financial incentive of $€ 350$ to a group training increased the proportion of successful quitters after 12 months from $26 \%$ to $41 \%$. This result demonstrates that with a relatively easy measure, the effectiveness of a smoking cessation group training can be significantly enhanced. Moreover, we found that the financial incentives were effective for participants with a low socioeconomic status, and our results showed that the incentives may be particularly attractive for people with a lower income.

This thesis also revealed several other success factors of a workplace group training that facilitated quit success, which are particularly relevant for smokers with a low socioeconomic status. ${ }^{6}$ For example, a training at the workplace is cost-free for employees, and regarded as convenient by them. This lowers the threshold to enroll in the smoking cessation training. Furthermore, the smoking cessation trainer shares important strategies on how to quit smoking and remain abstinent, and provides counselling for an extensive 
period of about 2 months. In addition, the group setting facilitates social support from colleagues and creates positive peer pressure, which helps to refrain from smoking.

The results of the current thesis provide evidence for financial incentives being an effective new method to stimulate smoking cessation. We also show that group trainings in a work setting are experienced as an effective way to quit smoking, and that the convenience of cost-free trainings at the work setting may make it easier for employees to participate in such a training. Workplace smoking cessation group trainings with financial incentives may thus help to decrease health disparities attributable to smoking if they are specifically aimed at smokers with a lower socioeconomic status.

\section{Target groups}

The results of the current thesis are relevant for several target groups. In the first place, it informs people who want to quit smoking on an intervention that can help them quit smoking. The results of the current thesis may convince smokers to enroll in a smoking cessation program with incentives if this is offered by their employer, or may motivate them to quit smoking in a community-based smoking cessation group training.

Secondly, the current results may inspire employers to offer smoking cessation group trainings with incentives to their employees. We are currently collaborating with VNO-NCW and MKB-Netherlands to disseminate smoking cessation group trainings with financial incentives among large branch organizations. Our results not only show that this method is effective to increase smoking cessation; it is also much appreciated by the employees. Additionally, our cost-effectiveness analysis offers more insight in the cost-effectiveness of financial incentives which facilitate the decision making process of implementation of smoking cessation trainings with financial incentives.

Thirdly, our study results are relevant for providers of smoking cessation counselling, such as smoking cessation coaches who offer community and company group trainings. Our research findings may stimulate smoking cessation counsellors to add financial incentives to their trainings in order to enhance their success rates, or to promote this idea to employers who organize a group training.

Fourthly, the results of the current thesis are relevant for health insurance companies, who have an interest in interventions that potentially reduce healthcare costs of their customers. Insurance companies offer collective insurance policies to organizations and can provide companies with a budget for health promotional interventions aimed at employees. Since January 2020, smoking cessation treatment is already fully reimbursed by all insurance companies. Based on the current thesis, insurers may decide to additionally reimburse financial incentives when used in the context of a smoking cessation training.

Lastly, our research informs the Dutch government on a new evidence-based approach to stimulate smoking cessation in the Netherlands, and has the interest of the ministry of Health, Welfare and Sport and the ministry of Foreign Affairs. Dependent on the scale of 
the implementation, our research could contribute to the aims of the National Prevention Agreement, which include effective and accessible smoking cessation counselling and smoke free companies, which should result in a reduction of smoking prevalence among adults to less than $5 \%$ of the population and $0 \%$ in youth and pregnant women by 2040 .

\section{Activities and products}

\section{Dissemination of research results through conferences and media appearances}

We have stimulated the dissemination of our research project in various ways. We have published nine research papers in international scientific journals and three in Dutch medical journals. We have additionally presented our research at national and international scientific conferences including World- and Europe meetings of the Society for Research on Nicotine and Tobacco (SRNT), the World Conference on Tobacco or Health (WCTOH), the Lisbon Addictions conference, the conference of the European Respiratory Society (ERS), and the annual conferences of the Dutch Network of Tobacco Researchers (NNvT). We furthermore issued press releases which have resulted in massive media attention, including several television appearances (including NOS News, RTL News, Hart van Nederland (SBS6), L1 News, and EditieNL (RTL4)), radio interviews (including Radio 1, 3FM, L1 radio, BNR News radio), newspaper articles (NRC, De Telegraaf, De Limburger, $A D$, Metro, and many local newspapers), and articles on various online platforms, which together have raised the public knowledge of the research project and results. The digital links to various news items are provided in the Appendix.

\section{National Prevention Agreement}

Our study was included in the National Prevention Agreement that was published by the Ministry of Health, Welfare and Sport, ${ }^{7}$ in which our intervention of group trainings with financial incentives was recommended as an effective method to stimulate smoking cessation.

\section{Collaborations with stakeholders to stimulate the implementation of financial incentives}

During the research project, we have collaborated with SineFuma, the largest provider of smoking cessation counselling in the Netherlands. Because our research has shown that financial incentives for smoking cessation can significantly increase quit success, SineFuma now advises their clients to implement financial incentives. In addition, SineFuma has included new information in their handbook for companies on how to organize a smoking cessation training, about applying financial incentives, and about effective communication methods to reach employees with a low socioeconomic status. Furthermore, we have advised two large hospitals on the implementation of group trainings with financial incentives for their employees. We also are working with two large health insurance companies and a health insurance collective on stimulating the implementation of group trainings with incentives among their corporate clients. Finally, during the research project we have collaborated with the Lung Alliance Netherlands, and our project was part of their National Action Program Chronic Lung Diseases. 


\section{Website, videos and posters}

We present the results of the research project on the effectiveness of financial incentives on a website that we developed as part of a follow-up implementation research project: http://www.samensterkerstoppen.nl. Our website informs employers and intends to convince them to offer their employees a smoking cessation group training with financial incentives. The information and materials that we provide on the website are focused on helping employers reach employees with a low socioeconomic status and motivating them to enroll in the smoking cessation group training. Among other things, our website provides information on reasons to stimulate smoking cessation and to use financial incentives, offers an overview of the costs and benefits of providing a smoking cessation group training with incentives, provides strategies to recruit employees, and answers on frequently asked questions. Moreover, employers can download posters that we developed to recruit employees. Our website also contains an animated video, which explains the advantages of providing smoking cessation group trainings with financial incentives. In addition, it includes a corporate video (see https://www.samensterkerstoppen.nl/ videos) that we filmed in one of the companies that participated in our research, in which employees explain how they experienced quitting smoking.

\section{Innovation}

Using financial incentives to simulate health behavior including smoking cessation is still very uncommon in the Netherlands. The current research project is the first in the Netherlands to conduct a large-scale cluster randomized trial to assess the effect of financial incentives on smoking cessation. In addition, international studies on financial incentives for smoking cessation have mostly investigated financial incentives without combining this with an extensive group smoking cessation program at the workplace. Therefore, this thesis provides new and valuable insights on this combination of incentives with group counseling. Moreover, little research has been performed on the cost-effectiveness of financial incentives for smoking cessation. The current thesis adds to the literature with a detailed trial-based economic evaluation of the cost-effectiveness of financial incentives to inform employers and policy makers.

\section{Planning}

A follow-up implementation research project is currently conducted to facilitate the implementation of financial incentives for smoking cessation in the Netherlands. This research project, running from December 2019 to December 2023, aims to develop a strategy to stimulate the implementation of smoking cessation group trainings with financial incentives in Dutch companies. The project is targeted specifically towards smokers with a lower socioeconomic status. It is funded by ZonMw and conducted by Maastricht University and IVO Research Institute. In order to achieve an effective nationwide dissemination and implementation of the intervention, it is essential to reach a large proportion of the companies in the Netherlands. This can only be accomplished if the implementation strategy will be developed together with, and 
supported and disseminated by, important stakeholders, including health insurance companies, trade organizations, occupational health and safety services, and providers of smoking cessation trainings. Therefore, various important stakeholders are actively involved in the development of our implementation strategy: Leiden University Medical Center, SineFuma, Rotterdam University of Applied Sciences, Lung Alliance Netherlands, RadboudUMC, Trimbos Institute, Centre for Motivation and Change, Heartbeat Ventures, HumanCapitalCare B.V., InGuide, Fire Brigade South-Limburg, social employment center SBCM, social work place MTB, Pharos, and Zuyderland Medical Center. 


\section{REFERENCES}

1. US Department of Health and Human Services. The health consequences of smoking-50 years of progress. In. a report of the Surgeon General. Vol 17. Atlanta, GA: US Department of Health and Human Services, Centers for Disease Control and Prevention, National Center for Chronic Disease Prevention and Health Promotion, Office on Smoking and Health; 2014.

2. World Health Organization. WHO global report on mortality attributable to tobacco. https:// apps.who.int/iris/bitstream/handle/10665/44815/9789241564434_eng.pdf. Published 2012. Accessed 20-03-2020.

3. Siahpush M, English D, Powles J. The contribution of smoking to socioeconomic differentials in mortality: results from the Melbourne Collaborative Cohort Study, Australia. J Epidemiol Community Health. 2006;60(12):1077-1079.

4. Jha P, Peto R, Zatonski W, Boreham J, Jarvis MJ, Lopez AD. Social inequalities in male mortality, and in male mortality from smoking: indirect estimation from national death rates in England and Wales, Poland, and North America. The Lancet. 2006;368(9533):367-370.

5. Brown T, Platt S, Amos A. Equity impact of European individual-level smoking cessation interventions to reduce smoking in adults: a systematic review. EurJPublic Health. 2014;24(4):551556.

6. van Wijk EC, Landais LL, Harting J. Understanding the multitude of barriers that prevent smokers in lower socioeconomic groups from accessing smoking cessation support: A literature review. Prev Med. 2019;123:143-151.

7. Ministerie van Volksgezondheid Welzijn en Sport. Nationaal preventieakkoord. Een gezonder Nederland. In:2018. 


\section{APPENDIX: Overview of media coverage}

\section{Television}

NOS Journaal (https://bit.ly/2J7yGvM - vanaf 21.38)

RTL Nieuws https://bit.ly/2pZdcJ5

Hart van Nederland (SBS 6) https://bit.ly/2ysFuQy

L1 https://bit.ly/2EvyCHN

Editie NL (RTL 4) https://bit.ly/2o1xPGG

\section{Radio}

Radio 1 https://bit.ly/2pYe14F

Radio 3FM https://bit.ly/2yNHZfQ

L1 Radio (https://bit.ly/2OwM9U9)

\section{Newspaper and online articles}

NOS nieuws https://bit.ly/2CScFkv

NRC Handelsblad/NRC next https://bit.ly/2J3hnfA

De Telegraaf (1) https://bit.ly/2CRCnWn

De Telegraaf (2) https://bit.ly/2J4s2X9

De Telegraaf (3) https://bit.ly/2VsOxeL

1 Limburg https://bit.ly/2pYaR0G

Elsevier Weekblad https://bit.ly/3bNwcjl

Nederlands Tijdschrift voor Geneeskunde (1) https://bit.ly/2V0Gbeq

Nederlands Tijdschrift voor Geneeskunde (2) https://bit.ly/35tLOGC

Following a press release that was published by Maastricht University, ANP news agency wrote an article that was distributed online via:

https://bit.ly/2ErsNeb Online news platform nu.nl

https://bit.ly/2QWxM89 Dagblad De Limburger (on Friday the newspaper dedicated the editorial column "Op de Korrel" to the research - https://bit.ly/2AjhfpA)

https://bit.ly/2AgroTI Algemeen Dagblad (ad.nl) - the same article was also distributed through the regional newspapers Tubantia, De Stentor, De Gelderlander, Eindhovens Dagblad, Brabants Dagblad, BN De Stem, Provinciale Zeeuwse Courant and de Gooi - and Eemlander.

https://bit.ly/2J5b5Mo Interview with Floor van den Brand and reactions of the employees of the agricultural cooperative Vitelia in the free newspaper Metro

https://bit.ly/2Owc1PH RTV Drenthe dedicates the "Statement of the Day" to the CATCH study: "Employers must help smokers to quit"

https://bit.ly/20x25FW Leeuwarder Courant and Dagblad van het Noorden

https://bit.ly/2OuRs6y Nederlands Dagblad and Reformatorisch Dagblad

https://bit.ly/2yOxt88 editorial commentary on the study in the Leids Dagblad

https://bit.ly/2RZgit3 online city newspapers Dagblad 010 in Rotterdam and Dagblad 070 in The Hague

https://bit.ly/2CS5t85 weekly magazine Panorama

https://bit.ly/2CsXLjx articles in trade magazines Voeding Nu and Medisch Contact 
https://bit.ly/2NQnsfU article on the website of LEF magazine that focuses on addicts in recovery and addiction care

https://bit.ly/2P3FSi8 websites of the TV programs Kassa (BNNVARA, NPO 1) and Zorg.nu (AVROTROS, NPO 1)

https://bit.ly/2CtxAcH Blogger Sylvia writes a comment on the research on the website of the magazine Libelle

https://bit.ly/2pYg5cV various online platforms such as Skipr, Welingelichte Kringen, Alles over HR, BlikopNieuws, Nationale Zorggids, Business Insider, nieuws.nl en MSN https://bit. ly/2PdHJ49

https://bit.ly/2Mmhe92 online platform XpertHR

https://bit.ly/2Wi7lqk Flemish weekly magazine Knack

https://bit.ly/2wRShuB Article in Algemeen Dagblad, which was also distributed through the regional newspapers Tubantia, De Stentor, De Gelderlander, Eindhovens Dagblad, Brabants Dagblad, BN De Stem, Provinciale Zeeuwse Courant and Dagblad van het Noorden. 
Summary 



\section{SUMMARY}

Tobacco smoking causes serious and often fatal conditions including cardiovascular disease, cancer and respiratory disease. Smoking is therefore a serious health threat and leads to high healthcare costs.

In $2018,22 \%$ of people aged 18 years and older smoked in the Netherlands. However, the percentage of smokers in Western countries is not equally divided among the population. Smoking is more prevalent among people with a low socioeconomic status than among people with a high socioeconomic status. In the Netherlands, $27 \%$ of the lower educated and $16 \%$ of the higher educated population smoked in 2018. Although the number of smokers in Western countries, including the Netherlands, has slowly declined in recent years, this gap in smoking prevalence has widened. Smoking is therefore the main cause of socioeconomic differences in health and mortality in Western countries.

There are several treatments that can effectively help people to quit smoking, such as behavioral support and pharmacological therapy. However, many interventions are less effective for people with a lower socioeconomic status. This is partly because there are barriers for them to receive treatment and to quit smoking, such as financial thresholds or a lack of social support for quitting smoking. To decrease smoking in society and to reduce the socioeconomic gap in smoking prevalence, it is therefore important to develop effective interventions that help people quit smoking. The aim of this dissertation was to investigate whether financial incentives for smoking cessation can enhance quit success among employees who participate in a workplace smoking cessation group training.

In this thesis, we answered the following research questions:

1. Can providing free smoking cessation treatment increase use of treatment, quit attempts and smoking cessation in people who smoke?

2. Are financial incentives for quit success on top of a workplace smoking cessation group training program effective at increasing smoking cessation in employees who smoke?

3. How does a workplace smoking cessation group training program with incentives increase quit success?

4. Are financial incentives on top of a workplace smoking cessation group training program a cost-effective intervention?

5. According to employers, what are the barriers and facilitators for the implementation of a smoking cessation group training program with incentives in workplaces? 
The first research question is addressed in Chapters 2 and 3. In Chapter 2, we describe a systematic review that examines the impact of reducing the costs for smokers or health care providers in using or giving smoking cessation treatments. The results showed that financial interventions for smokers led to more use of smoking cessation treatments, more quit attempts, and more successful quitters. There was no consistent evidence for an effect of financial interventions targeting healthcare providers.

In chapter 3, we describe a questionnaire study among representative cohorts of 1164 Dutch smokers and 768 smokers from the United Kingdom (UK). The aim was to investigate whether free or lower-cost smoking cessation medication was a trigger for respondents to think about quitting smoking. The results showed that smokers from the Netherlands and the UK who mentioned this trigger were more likely to actually use medication during a quit attempt. In addition, in the UK only, we saw an association between quit attempts and mentioning free or lower-cost smoking cessation medication as a trigger to think about quitting smoking. We found no statistically significant associations with smoking cessation success. Based on the two studies described above, it can be concluded that funding smoking cessation treatments for smokers can have a positive impact on the number of people using these treatments, and may increase quit attempts and quit success. Therefore, reimbursing smoking cessation treatments can be a good strategy to promote smoking cessation in society.

We address the second research question in Chapters 4 and 5: Are financial incentives for quit success on top of a workplace smoking cessation group training program effective at increasing smoking cessation in employees who smoke? Chapter 4 describes the protocol of a cluster randomized controlled trial (RCT), the results of which we present in chapter 5 . In the study, we evaluated the effect of adding financial incentives for smoking cessation to a workplace group smoking cessation training program on abstinence from smoking. The survey involved 604 smoking employees from 61 Dutch companies. All participants followed a smoking cessation group training program at the workplace, consisting of 7 weekly 90 -minute sessions. In half of the companies, employees who successfully quit smoking also earned financial incentives in the form of digital gift certificates with a total value of $€ 350$. In the other half of the companies, employees only attended a group training and were not rewarded for quit success. The results showed that after 12 months in the group of employees who only attended smoking cessation training, $26 \%$ had stopped smoking. In the group of employees who also earned financial incentives, $41 \%$ had stopped; a statistically significant increase. An important finding was that the incentives also had a positive effect on quit success among participants with a low socioeconomic status. The results of this study show that financial incentives can substantially increase the effectiveness of a smoking cessation group training. This finding can motivate employers to organize a smoking cessation training with financial incentives to support their employees in quitting smoking.

The studies in chapters 6, 7 and 8 answer the third research question: How does a workplace smoking cessation group training program with incentives increase quit success? In chapter 6, we describe a qualitative study in which we interviewed 24 participants who participated in a smoking cessation group training at the workplace 
and earned gift vouchers for quit success. The purpose of the interviews was to find out the factors that, according to the interviewees, had contributed positively to smoking cessation. According to the participants, important success factors were the accessibility of a workplace training, the social support and peer pressure of colleagues, support from family members, strategies for not smoking that were learned during the training, and personal motivation to quit smoking. The incentives were considered a nice added bonus, but not the reason for quitting smoking. Interviewees with a low income found the incentives more attractive. This study shows that a smoking cessation group training with financial incentives at the workplace is a pleasant way to quit smoking, according to participants.

In chapter 7, we investigated the influence of smoking behavior and social support of persons in the social environment on the quit success of employees who participated in a group smoking cessation training at the workplace by means of a questionnaire study. We found that social support from colleagues was positively associated with quit success immediately after training completion and after 12 months. Partner support was also positively associated with quit success, but only in the short term. In addition, the smoking behavior of the partner was associated with quit success; participants without a partner or with an (ex-) smoker as a partner quit smoking less often. Finally, there was a negative relationship between having more smokers in the immediate social environment and quit success. These results underline the important influence that the social environment has on smoking cessation success. It is therefore important not only to look at the individual who wants to quit smoking, but also to include his or her social environment in the attempt to quit.

In chapter 8, we performed a path analysis with the aim of investigating how financial incentives can lead to smoking cessation success. We tested a model in which we examined the effect of financial incentives via the mediators: training evaluation, medication use, use of nicotine substitutes, attitudes, self-efficacy and social influences, on quit success. The results showed that personal incentive sensitivity did not affect the relationship between incentives and quit success. The effect of financial incentives on quit success was mediated by higher self-efficacy and was associated with higher medication use. The latter implies that it may also be important to offer smoking cessation medication along with interventions that provide financial incentives.

We investigated the fourth research question, whether financial incentives for smoking cessation are cost effective, in an economic evaluation described in chapter 9 . The analysis from a societal perspective, which included all costs over the 14 months of the study, resulted in an incremental cost-effectiveness ratio (ICER) for a training with financial incentives compared to a training without incentives of $€ 11,546$. If only the costs that are relevant to the employer were included, the ICER was $€ 5,686$. The analysis based on a lifetime perspective showed an ICER of $€$ 1,249 per QALY (an extra year in good health). This amount falls well within the "willingness to pay" (the amount that the company is willing to pay for a QALY), which is set at $€ 20,000$; demonstrating that financial incentives can be a cost effective intervention. 
We answer the last research question in chapter 10: according to employers, what are the barriers and facilitators for the implementation of a smoking cessation group training program with incentives in workplaces? Here we describe a qualitative study in which we interviewed 18 employers. The knowledge that we gained from the interviews on the promoting and hindering factors is necessary to be able to develop a targeted strategy to stimulate the implementation of stop-smoking training with incentives. The following action points emerged from these interviews: 1 ) train employers in how to reach and convince their employees to participate in a smoking cessation group training, 2) explain to employers that their employees will not consider financial incentives unfair or come up with some form of alternative (non-financial) incentives, 3 ) explain the cost-effectiveness of a smoking cessation group training with financial incentives, and 4) make smoking cessation a part of the organization's existing health policy.

This dissertation ends with Chapter 11, in which we discuss the results of the studies described above and compare them with the literature. In addition, methodological issues are discussed and recommendations are made for further research and for future policies.

For further research, we make the following recommendations:

1. determine how financial incentives should be designed to be effective. This includes the amount of the incentives, the schedule in which the incentives are provided and the type of incentive;

2. consider whether incentives for smoking cessation can also be used effectively in an environment other than the workplace, such as in healthcare, so that people without work can also be encouraged to stop smoking;

3. investigate the cost-effectiveness of incentives in combination with other forms of smoking cessation treatments such as individual treatment and e-health interventions.

Our policy recommendations are the following:

1. develop national policies that make employers accountable for the structural provision of smoking cessation support to their employees;

2. expand the smoking ban in public areas and raise tobacco product excise taxes;

3. launch mass media campaigns to inform the public that smoking cessation treatments are fully reimbursed under basic health insurance and to show where people can go for treatment. In addition, media campaigns are needed to change the societal view on smoking so that stigmatization of smokers is reduced and there is more support for research on smoking cessation interventions such as financial incentives;

4. create accessible smoking cessation treatments by increasing the provision of treatment by healthcare providers including GPs, whereby GPs take a more active role in discussing smoking cessation with their patients. 
Samenvatting 



\section{SAMENVATTING}

Het roken van tabak veroorzaakt ernstige en vaak dodelijke aandoeningen waaronder hart- en vaatziekten, kanker en luchtwegaandoeningen. Roken is daarom een ernstige bedreiging voor de gezondheid en leidt tot hoge zorgkosten.

In Nederland rookte in 2018 22\% van de personen van 18 jaar en ouder. Het percentage rokers is in westerse landen echter niet gelijk verdeeld over de bevolking. Er wordt aanzienlijk meer gerookt onder personen met een lage sociaaleconomische status dan onder personen met een hoge sociaaleconomische status. In Nederland rookte in 2018 $27 \%$ van de lager opgeleiden en $16 \%$ van de hoger opgeleide bevolking. Hoewel het aantal rokers in westerse landen, waaronder Nederland, langzaam is gedaald in de afgelopen jaren, is deze kloof in rookprevalentie gegroeid. Roken is hierdoor de grootste veroorzaker van sociaaleconomische verschillen in gezondheid en sterfte in westerse landen.

Er zijn verschillende behandelingen die mensen effectief kunnen helpen om te stoppen met roken, bijvoorbeeld gedragsmatige ondersteuning en medicatie. Echter, veel interventies zijn minder effectief voor mensen met een lagere sociaaleconomische status. Dit wordt onder andere veroorzaakt doordat er barrières voor hen zijn om een behandeling te ontvangen en om te stoppen met roken, bijvoorbeeld financiële drempels of een gebrek aan sociale steun voor het stoppen met roken. Om roken in de samenleving te verminderen en de sociaaleconomische kloof in rookprevalentie te verkleinen is het dus belangrijk om effectieve interventies te ontwikkelen die mensen helpen stoppen met roken. Het doel van deze dissertatie was om te onderzoeken of financiële beloningen voor stoppen met roken het stopsucces kunnen verhogen bij rokende werknemers die deelnemen aan een stoppen-met-roken groepstraining op de werkplek.

In dit proefschrift beantwoordden we de volgende onderzoeksvragen:

1. Kan het aanbieden van gratis stoppen-met-rokenbehandelingen aan rokers het gebruik van deze behandeling verhogen, stoppogingen stimuleren en stopsucces verhogen?

2. Zijn financiële beloningen voor stoppen met roken bij werknemers die deelnemen aan een groepstraining op de werkplek effectief om het stopsucces te vergroten?

3. Hoe vergroot een stoppen-met-rokengroepstraining met beloningen op de werkplek het stopsucces?

4. Is het toevoegen van financiële beloningen aan een trainingsprogramma voor stoppen met roken op de werkplek een kosteneffectieve interventie?

5. Wat zijn volgens werkgevers de belemmerende en bevorderende factoren voor de uitvoering van een stoppen-met-roken groepstraining met beloningen op de werkplek? 
De eerste onderzoeksvraag komt aan bod in hoofdstuk 2 en 3. In hoofdstuk 2 beschrijven we een systematische review die onderzoekt wat de invloed is van het reduceren van de kosten voor rokers of zorgverleners voor het gebruikmaken van of het geven van stoppen-met-rokenbehandelingen. De resultaten lieten zien dat financiële interventies voor rokers leidden tot meer gebruik van behandelingen voor stoppen met roken, tot meer stoppogingen, en tot meer succesvolle stoppers. Er was geen consistent bewijs voor een effect van financiële interventies gericht op zorgverleners.

In hoofdstuk 3 beschrijven we een vragenlijstonderzoek onder representatieve cohorten van 1164 Nederlandse rokers en 768 rokers uit het Verenigd Koninkrijk (VK). Het doel was om te onderzoeken of gratis of goedkopere stoppen-met-rokenmedicatie voor respondenten een trigger was om te overwegen om te stoppen met roken. De resultaten lieten zien dat rokers uit Nederland en het VK die deze trigger noemden vaker ook daadwerkelijk medicatie gebruikten tijdens een stoppoging. Daarnaast zagen we alleen in het VK een associatie van aantal stoppogingen met het noemen van gratis of goedkopere stoppen-met-rokenmedicatie als een trigger om te stoppen met roken. We vonden geen statistisch significante associaties met stopsucces. Op basis van de twee hierboven beschreven onderzoeken kan geconcludeerd worden dat het financieren van stoppenmet-rokenbehandelingen voor rokers een positieve invloed kan hebben op het aantal mensen dat gebruik maakt van deze behandelingen, en stoppogingen en stopsucces kan verhogen. Daarom kan het vergoeden van stoppen-met-rokenbehandelingen een goede strategie zijn om stoppen met roken in de samenleving te stimuleren.

De tweede onderzoeksvraag wordt behandeld in hoofdstuk 4 en 5: zijn financiële beloningen voor succesvol stoppen met roken bovenop een groepstraining op de werkplek effectief om het stopsucces te vergroten van rokende werknemers? Hoofdstuk 4 beschrijft het protocol van een cluster-gerandomiseerde gecontroleerde trial (RCT), waarvan we in hoofdstuk 5 de resultaten presenteren. In het onderzoek evalueerden we het effect van financiële beloningen voor stopsucces in combinatie met een stoppenmet-roken groepstraining op de werkplek op stoppen met roken. Aan het onderzoek namen 604 rokende werknemers deel van 61 Nederlandse bedrijven. Allen volgden een stoppen-met-roken groepstraining op de werkplek, bestaande uit 7 wekelijkse sessies van 90 minuten. In de helft van de bedrijven verdienden werknemers als zij succesvol stopten met roken daarnaast financiële beloningen in de vorm van digitale cadeaubonnen met een totale waarde van $€ 350$. In de andere helft van de bedrijven volgden werknemers alleen de groepstraining en kregen ze geen beloning voor stopsucces. De resultaten lieten zien dat na 12 maanden in de groep werknemers die alleen een stoppen-metrokentraining volgden $26 \%$ gestopt was met roken. In de groep werknemers die daarnaast ook financiële beloningen verdienden was $41 \%$ gestopt; een statistisch significante verhoging. Een belangrijke bevinding was dat de beloningen ook een positief effect hadden op stopsucces bij deelnemers met een lage sociaaleconomische status. De resultaten van dit onderzoek tonen aan dat financiële beloningen de effectiviteit van een stoppen-met-roken groepstraining substantieel kunnen verhogen. Deze kennis kan werkgevers motiveren om een stoppen-met-rokentraining met financiële beloningen te organiseren om hun werknemers te ondersteunen bij het stoppen met roken. 
De studies in hoofdstuk 6, 7 en 8 geven antwoord op de derde onderzoeksvraag: hoe vergroot een stoppen-met-roken groepstraining met beloningen op de werkplek het stopsucces? In hoofdstuk 6 beschrijven we een kwalitatief onderzoek waarin we 24 deelnemers interviewden die deel hadden genomen aan een stoppen-met-roken groepstraining op de werkplek en daarbij cadeaubonen verdienden voor stopsucces. Het doel van de interviews was om de factoren te achterhalen die volgens de geïnterviewden positief hadden bijgedragen aan het stoppen met roken. Volgens de deelnemers waren belangrijke succesfactoren de laagdrempeligheid van een training op de werkplek, de sociale steun en groepsdruk van collega's, steun van familieleden, strategieën om niet te roken die men tijdens de training leerde, en persoonlijke motivatie om te stoppen. De beloningen werden gezien als een leuke bijkomstigheid, maar niet als de reden om te stoppen met roken. De beloningen werden wel aantrekkelijker gevonden door geïnterviewden met een laag inkomen. Dit onderzoek toont aan dat een training met beloningen op de werkplek volgens deelnemers een prettige manier is om te stoppen met roken.

In hoofdstuk 7 onderzochten we door middel van een vragenlijstonderzoek de invloed van het rookgedrag en de sociale steun van personen in de sociale omgeving op het stopsucces van de werknemers die hadden deelgenomen aan de stoppen-met-rokentraining. We vonden dat sociale steun van collega's positief geassocieerd was met stopsucces direct na afronding van de training en na 12 maanden. Steun van de partner was ook positief geassocieerd met stopsucces maar alleen op de korte termijn. Ook het rookgedrag van de partner was geassocieerd met stopsucces; deelnemers zonder partner of met een (ex-) roker als partner stopten minder vaak met roken. Er was tot slot een negatief verband tussen meer rokers in de directe sociale omgeving hebben en stopsucces. Deze resultaten onderschrijven de belangrijke invloed die de sociale omgeving op stopsucces heeft. Het is daarom belangrijk om niet alleen naar het individu te kijken dat wil stoppen met roken, maar ook zijn of haar sociale omgeving bij de stoppoging te betrekken.

In hoofdstuk8voerden weeen padanalyse uitmetals doel om teonderzoeken hoefinanciële beloningen kunnen leiden tot stopsucces. We testten een model waarin we het effect van financiële beloningen via de mediatoren trainingsevaluatie, medicatiegebruik, gebruik van nicotinevervangers, attitudes, eigen effectiviteit en sociale invloeden op stopsucces onderzochten. De resultaten toonden aan dat persoonlijke beloningsgevoeligheid geen invloed had op het verband tussen beloningen en stopsucces. Het effect van financiële beloningen op stopsucces werd gemedieerd door een hogere eigen effectiviteit en was geassocieerd met een hoger gebruik van medicatie. Dit laatste impliceert dat het bij interventies met beloningen ook belangrijk kan zijn om stoppen-met-rokenmedicatie aan te bieden.

We onderzochten de vierde onderzoeksvraag, of financiële beloningen voor stoppen met roken kosteneffectief zijn, in een economische evaluatie die beschreven wordt in hoofdstuk 9. De analyse vanuit een maatschappelijk perspectief, waarin alle kosten werden meegerekend gedurende de 14 maanden dat het onderzoek in beslag nam, resulteerde in een incrementele kosten-effectiviteitsratio (ICER) voor een training met beloningen vergeleken met een training zonder beloningen van $€ 11.546$. Als alleen de 
kosten werden meegerekend die relevant zijn voor de werkgever was de ICER €5.686. De analyse waarin er werd uitgegaan van een levenslang perspectief toonde een ICER van $€ 1.249$ per QALY (een extra levensjaar in goede gezondheid). Dit bedrag valt ruim binnen de 'willingness to pay' (het bedrag dat de maatschappij bereid is om te betalen voor een QALY) die is vastgesteld op $€ 20.000$; wat aantoont dat financiële beloningen een kosteneffectieve interventie kunnen zijn.

Delaatste onderzoeksvraag beantwoorden we in hoofdstuk 10: wat zijn volgens werkgevers de belemmerende en bevorderende factoren voor de uitvoering van een stoppenmet-roken groepstraining met beloningen op de werkplek? Hier beschrijven we een kwalitatieve studie waarin we 18 werkgevers interviewden. De kennis die we vergaarden uit de interviews over deze belemmerende en bevorderende factoren is nodig om een gerichte strategie te kunnen ontwikkelen waarmee de implementatie van stoppen-metrokentrainingen met beloningen gestimuleerd kan worden. Uit deze interviews kwamen de volgende actiepunten naar voren: 1 ) werkgevers trainen in hoe ze hun werknemers kunnen bereiken en overtuigen om deel te nemen aan de groepstraining, 2) werkgevers uitleggen dat hun werknemers de financiële beloningen niet oneerlijk zullen vinden of een vorm van alternatieve (niet-financiële) beloningen bedenken, 3) de kosteneffectiviteit van groepstrainingen en financiële beloningen uitleggen, en 4) stoppen met roken een onderdeel maken van het bestaande gezondheidsbeleid van de organisatie.

Deze dissertatie eindigt met Hoofdstuk 11, waarin we de resultaten van de hierboven beschreven studies bediscussiëren en vergelijken met de literatuur. Daarnaast worden er methodologische zaken besproken en worden er aanbevelingen gedaan voor verder onderzoek en voor toekomstig beleid.

Voor verder onderzoek doen we de volgende aanbevelingen:

1. bepalen hoe een beloning vormgegeven moet worden om effectief te zijn. Hieronder valt onder andere de hoogte van de beloning, het schema waarin de beloningen verstrekt worden en het type beloning;

2. bekijken of beloningen voor stoppen met roken ook effectief ingezet kunnen worden in een andere omgeving dan de werkplek, zoals de gezondheidszorg, zodat ook mensen zonder werk gestimuleerd kunnen worden om te stoppen met roken;

3. onderzoeken wat de kosteneffectiviteit is van beloningen in combinatie met andere vormen van stoppen-met-rokenbehandelingen zoals individuele behandeling en e-health interventies. 
Onze beleidsaanbevelingen zijn de volgende:

1. het ontwikkelen van nationaal beleid waarin werkgevers verantwoordelijk worden gemaakt voor het structureel aanbieden van stoppen-met-rokenondersteuning aan hun werknemers;

2. het uitbreiden van het rookverbod in publieke ruimtes en het verhogen van tabaksaccijnzen;

3. het opzetten van massamediacampagnes waarin de bevolking wordt geïnformeerd dat stoppen-met-rokenbehandelingen volledig vergoed worden vanuit de basisverzekering en waarin wordt verteld waar mensen voor behandeling terecht kunnen. Daarnaast zijn mediacampagnes nodig om de maatschappelijke kijk op roken te veranderen zodat het stigmatiseren van rokers vermindert en er meer draagvlak komt voor het investeren in stoppen-met-rokeninterventies zoals financiële beloningen;

4. toegankelijke stoppen-met-rokenbehandelingen creëren door het aanbod bij onder andere huisartsen te verhogen, waarbij huisartsen een actievere rol nemen in het bespreken van stoppen met roken met hun patiënten. 

Dankwoord 



\section{DANKWOORD}

Dit proefschrift was niet tot stand gekomen zonder de bijdrage van anderen. Hierbij wil ik graag iedereen bedanken die heeft geholpen bij de totstandkoming van dit proefschrift.

Ten eerste wil ik graag de bedrijven bedanken die zich hebben aangemeld om deel te nemen aan dit onderzoek, en wil ik de werknemers bedanken die de uitdaging zijn aangegaan om te stoppen met roken en mee te doen met het onderzoek, en tijd hebben gemaakt om de vragenlijsten in te vullen en deel te nemen aan de metingen.

Daarnaast wil ik graag Marcel, Maaike, Debbie, en de andere toppers van SineFuma bedanken voor de prettige samenwerking, zonder welke dit onderzoek niet mogelijk was geweest. Uiteraard wil ik de stoppen-met-rokentrainers van SineFuma ook hartelijk bedanken voor het geven van groepstrainingen waarvoor wij van de deelnemers veel complimenten hebben gehad.

Mijn dank gaat uit naar KWF Kankerbestrijding, de subsidieverstrekker van dit onderzoek. De benaderbaarheid en de prettige communicatie met medewerkers van KWF heb ik erg gewaardeerd tijdens dit project. Daarnaast wil ik graag ZonMw bedanken voor het financieren van een vervolgonderzoek waardoor we de implementatie van financiële incentives kunnen bevorderen zodat er hopelijk meer mensen in Nederland ondersteuning kunnen krijgen bij het stoppen met roken.

Speciale dank gaat uit naar mijn promotieteam. Onno, Gera, Bjorn en Niels, bedankt voor de kans om op dit mooie project te kunnen promoveren en voor jullie begeleiding de afgelopen jaren. Jullie stonden altijd voor me klaar als het nodig was en jullie positieve manier van begeleiden gaf mij veel vertrouwen.

Onno, ondanks jouw volle agenda en vele projecten had je altijd tijd voor overleg, lukte het je om snel feedback te geven op mijn stukken en bleef je goed op de hoogte van de vorderingen in het project. Ook jouw positieve stijl van begeleiden heb ik als heel prettig ervaren.

Gera, ik mag me erg gelukkig prijzen met jouw goede begeleiding. Ik kon altijd bij jou terecht met vragen, en als ik even vastliep was een overleg met jou genoeg om weer verder te kunnen. Door jouw kennis en oog voor detail heb je mij geholpen om een beter onderzoeker te worden en om dit proefschrift naar een hoger niveau te tillen.

Bjorn, het was voor mij erg fijn om jou als begeleider in mijn team te hebben. Jij hielp mij om de puntjes op i te zetten bij het schrijven van de stukken in dit proefschrift, en was onmisbaar bij de statistische analyse van de onderzoeksdata. Daarnaast was het natuurlijk ook leuk om het behalve over statistiek met jou ook over wielrennen te kunnen hebben.

Niels, ook op afstand had jij altijd goede suggesties waar ik mee verder kon, hartelijk dank daarvoor. 
Ook wil ik graag de beoordelingscommissie van dit proefschrift bedanken voor het lezen en beoordelen van het manuscript: Prof. dr. Andre Knottnerus, Prof. dr. Catherine Bolman, Prof. dr. Johan Mackenbach, Prof. dr. Nanne de Vries en Prof. dr. Hein de Vries. Tevens wil ik mijn dankbaarheid uitspreken richting de leden van de corona: Prof. dr. André Knottnerus, Prof. dr. Jan Degryse, Prof. dr. Nanne de Vries, Prof. dr. Angelique de Rijk en Marieke Helmus.

Bij het uitvoeren en analyseren van dit onderzoek heb ik hulp gehad van vele collega's, waarvoor ik erg dankbaar ben. Lotte, jouw bijdrage heeft een enorm belangrijke rol gespeeld in het succes van dit onderzoek. Door jouw overtuigingskracht en volharding is het gelukt om genoeg bedrijven te rekruteren voor dit onderzoek. Daarnaast ben je stad en land afgereisd om metingen uit te voeren bij de deelnemers. Zonder jouw hulp was dit project niet geslaagd, en was het bovendien een stuk minder gezellig geweest.

Mascha en Karin Aretz, bedankt dat jullie altijd bereid waren om te helpen met praktische zaken, het begeleiden van WESP-studenten, en bedankt voor jullie hulp bij de enorme klus van het opschonen van de databestanden. Daniel Kotz, bedankt voor jouw uitgebreide suggesties bij het schrijven van het onderzoeksprotocol voor de medisch-ethische commissie, en voor het gezelschap tijdens congressen samen met Sabrina en Melanie.

Stagiair(e)s Puck, Mathijs en Lisa, bedankt voor jullie enthousiaste inzet tijdens de onderzoeksstage en voor jullie bijdragen aan de artikelen in dit proefschrift.

Ikwil ookgraag de verscheidene co-auteurs bedanken voor hun bijdrage aan de verschillende artikelen die in dit proefschrift zijn opgenomen. Karin Hummel, bedankt voor je goede suggesties voor het artikel en voor de leuke tijd die we gehad hebben tijdens het congres in Oslo. Silvia, bedankt voor de colleges die ik bij jou kon volgen over economische evaluaties. Math, bedankt voor de prettige samenwerking en jouw expertise die een belangrijke rol heeft gespeeld bij de totstandkoming van het artikel. Tessa, bedankt voor jouw bijdrage als co-auteur en voor de leuke dagen tijdens de cursus in Maastricht.

Collega's van de afdeling huisartsgeneeskunde, bedankt voor de goede sfeer op de afdeling en de kletsmomenten in het keukentje, en uiteraard de taarten bij Heel Huisartsgeneeskunde Bakt. Ook de lunchwandelingen waren altijd welkom en hielpen mij om weer fris aan de slag te kunnen. Ik hoop dat we elkaar snel weer kunnen zien op de afdeling en de gezellige gewoontes blijven vasthouden!

Kamergenootjes Marjolein en Anouk, bedankt voor de gezelligheid in onze kamer. Vanwege jullie is het altijd leuk om naar werk te komen, met het huidige thuiswerken mis ik jullie gezelschap! Kirsten, ik ben blij dat je nog steeds mijn overbuurvrouw bent, en dat we ondanks dat de afstand iets groter is we nog steeds samen kunnen lunchen. Maartje, zonder jou was ik waarschijnlijk niet in Maastricht beland, en mijn assistentschap bij de gezonde basisschool was de aanleiding tot dit promotietraject. Ik ben heel blij met jouw vriendschap en ik bewonder jouw niet aflatende energie en enthousiasme.

Tot slot wil ik mijn familie en vrienden bedanken voor hun steun. Lieve Peter, je 'kent me al langer dan vandaag', en daar ben ik heel gelukkig mee. 
Publications 



\section{INTERNATIONAL PUBLICATIONS}

Van den Brand FA, Nagelhout GE, Winkens B, Chavannes NH, van Schayck OCP, Evers SMAA. Cost-effectiveness and cost-utility analysis of a work-place smoking cessation intervention with and without financial incentives. Addiction. 2020;115(3):534-545.

Van den Brand FA, Candel MJJM, Nagelhout GE, Winkens B, van Schayck CP. How Financial Incentives Increase Smoking Cessation: A Two-Level Path Analysis. [published online ahead of print, 2020 Jan 29]. Nicotine Tob Res. 2020;ntaa024. doi:10.1093/ntr/ntaa024

Van den Brand FA, Nagtzaam P, Nagelhout GE, Winkens B, van Schayck CP. The Association of Peer Smoking Behavior and Social Support with Quit Success in Employees Who Participated in a Smoking Cessation Intervention at the Workplace. Int J Environ Res Public Health. 2019;16(16):2831.

Van den Brand FA, Magnée T, de Haan-Bouma L, et al. Implementation of Financial Incentives for Successful Smoking Cessation in Real-Life Company Settings: A Qualitative Needs Assessment among Employers. Int J Environ Res Public Health. 2019;16(24):5135.

Van den Brand FA, Nagelhout GE, Hummel K, Willemsen MC, McNeill A, van Schayck OCP. Does free or lower cost smoking cessation medication stimulate quitting? Findings from the International Tobacco Control (ITC) Netherlands and UK Surveys. Tob Control. 2019;28(Suppl 1):s61.

Van den Brand FA, Nagelhout GE, Winkens B, Chavannes NH, van Schayck OCP. Effect of a workplace-based group training program combined with financial incentives on smoking cessation: a cluster-randomized controlled trial. The Lancet Public Health. 2018;3(11):e536e544. doi:10.1016/S2468-2667(18)30185-3

Van den Brand FA, Dohmen LME, Van Schayck OCP, Nagelhout GE. 'Secretly, it's a competition': a qualitative study investigating what helped employees quit smoking during a workplace smoking cessation group training program with incentives. BMJ Open. 2018;8(11):e023917.

Van den Brand FA, Nagelhout GE, Reda AA, et al. Healthcare financing systems for increasing the use of tobacco dependence treatment. Cochrane Database Syst Rev. 2017(9).

Van den Brand FA, Nagelhout GE, Winkens B, et al. The effect of financial incentives on top of behavioral support on quit rates in tobacco smoking employees: study protocol of a cluster-randomized trial. BMC Public Health. 2016;16(1):1056. 


\section{NATIONAL PUBLICATIONS}

Nagtzaam PF, van den Brand F, Nagelhout G, Winkens B, Chavannes N, van Schayck O. Steun op het werk bij stoppen met roken. Huisarts en wetenschap 2020. doi:10.1007/ s12445-020-0593-x

Van den Brand FA, G Nagelhout, B Winkens, N Chavannes, O van Schayck. Stoppen met roken op het werk. Huisarts \& Wetenschap 2019, 62 (5), 19-22.

Dohmen, L.M.E.; Van den Brand, F.A.; Van Schayck, C.P.; Nagelhout, G.E. Stoppen met roken met je collega's én een cadeaubon als het lukt. Ned Tijdschr Geneeskd. 2019;163:D3833. 
Curriculum Vitae 



\section{CURRICULUM VITAE}

Floor van den Brand was born on December 3rd 1986 in Oosterhout NB, The Netherlands. After graduating secondary school at St. Oelbert Gymnasium in Oosterhout, she started her studies in Health Sciences in 2005 at Maastricht University with specializations Movement Sciences and Health Promotion. After receiving her Bachelor's degree, she continued to study at Maastricht University and enrolled in the Master program Biology of Human Performance and Health, followed by a second Bachelor's degree in Psychology at Maastricht University. After she finished her studies in 2013, she worked as a research assistant at the department of Health Promotion and the Department of Family Medicine at Maastricht University. In December 2015 she started a $\mathrm{PhD}$ research project on the effect of financial incentives

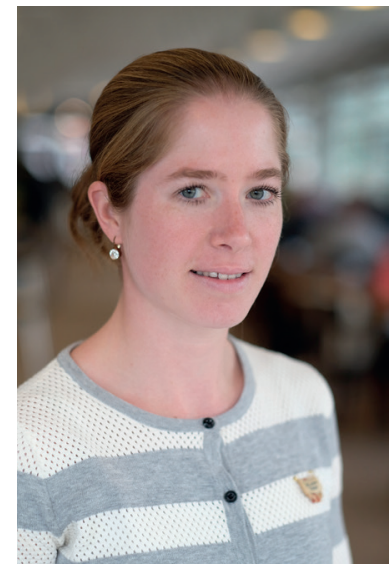
on smoking cessation at the Department of Family Medicine, supervised by Onno van Schayck, Gera Nagelhout, Bjorn Winkens and Niels Chavannes. Floor currently works as a post-doctoral researcher at the Department of Family Medicine on the implementation of financial incentives for smoking cessation.

In her free time, Floor enjoys cycling, running and swimming, and likes to compete in triathlons. She also teaches bootcamp and strength \& conditioning classes at the Maastricht University Sports Center. 

